\title{
MSER Exploratory Research: Implementations, Virtual Laboratory Development, and Parameterization Analysis
}

\author{
A Dissertation \\ Presented to \\ the faculty of the School of Engineering and Applied Science \\ University of Virginia
}

\author{
in partial fulfillment \\ of the requirements for the degree \\ Doctor of Philosophy \\ by
}

Sung Nam Hwang

May 2017 


\title{
APPROVAL SHEET
}

\author{
This Dissertation \\ is submitted in partial fulfillment of the requirements \\ for the degree of \\ Doctor of Philosophy
}

Author Signature:

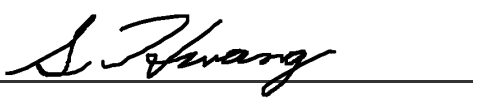

This Dissertation has been read and approved by the examining committee:

Advisor: Prof. K. Preston White, Jr

Committee Member: Prof. Michael C. Smith

Committee Member: Prof. James W. Lark III

Committee Member: Prof. William T. Scherer

Committee Member: Dr. Paul J. Sanchez

Committee Member:

Accepted for the School of Engineering and Applied Science:

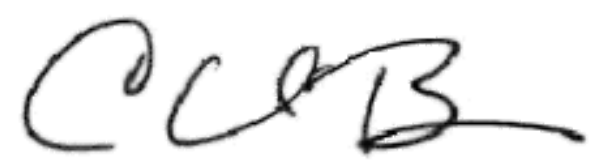

Craig H. Benson, School of Engineering and Applied Science

May 2017 


\section{TABLE OF CONTENTS}

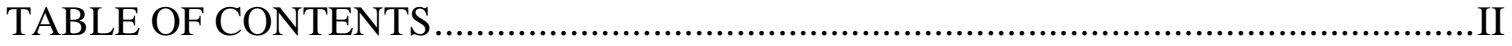

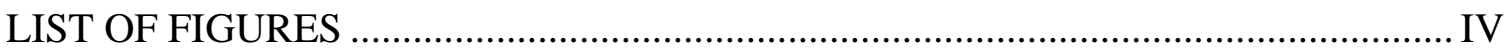

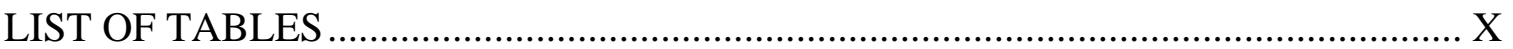

Acknowledgement ...................................................................................................

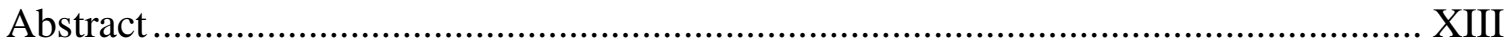

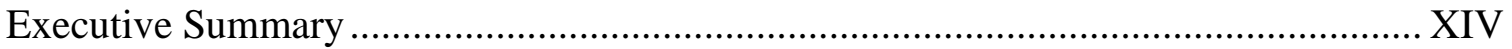

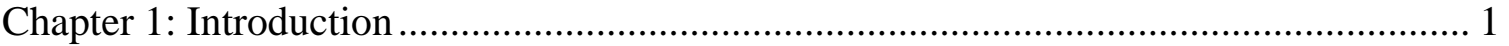

1.1 The Importance and Ubiquity of Discrete-Event, Stochastic Simulation ................................ 1

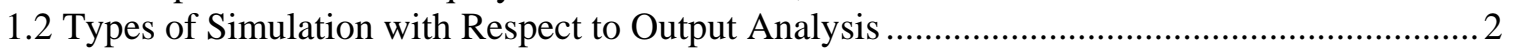

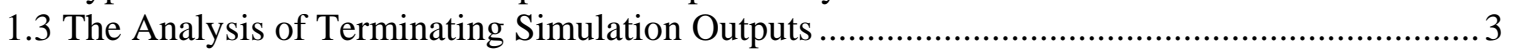

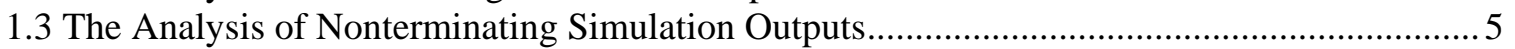

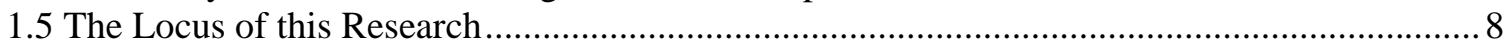

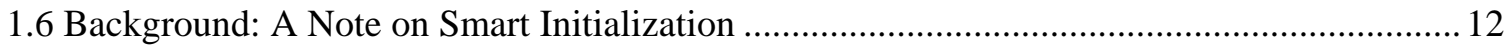

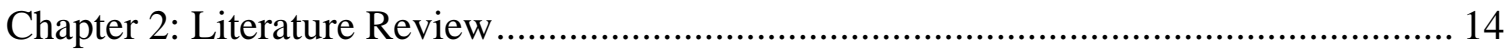

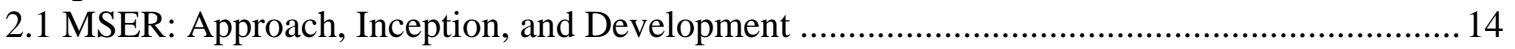

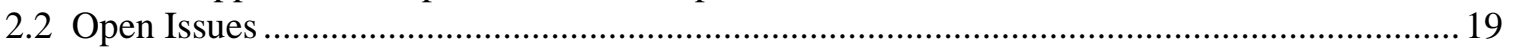

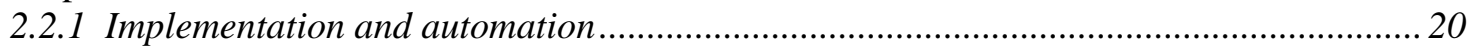

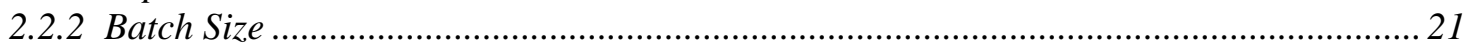

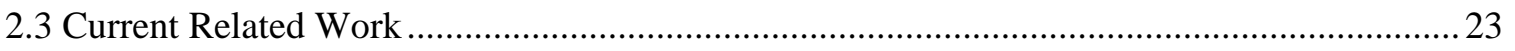

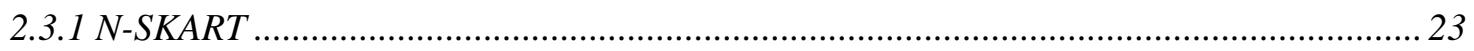

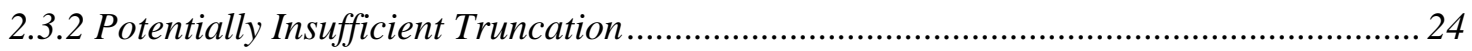

2.4 Optimal Analysis for the Mean of a Simulation Output ...................................................... 25

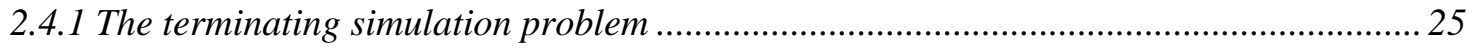

2.4.2 The steady-state simulation problem: replication/deletion approach .............................2. 27

2.4.3 The steady-state simulation problem: batch means approach ........................................28

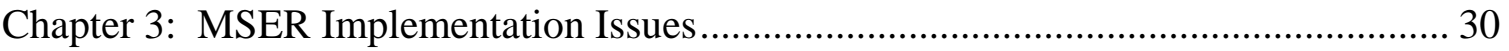

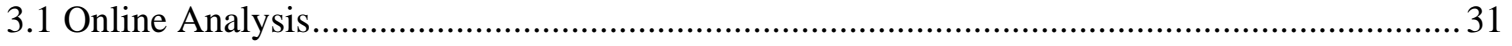

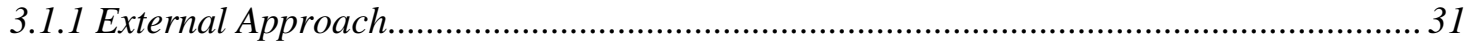

3.1.1.1 How to build DLL linked with ProModel (MedModel)............................................................

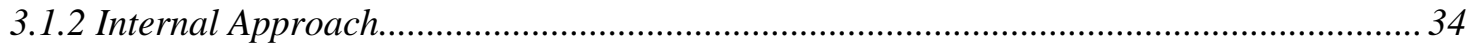

3.1.2.1 Software development environments ....................................................................................34

3.1.2.2 Current Features for Dealing with the Start-up Problem............................................................35

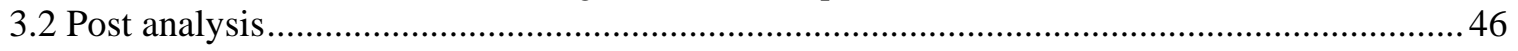

3.2.1 R

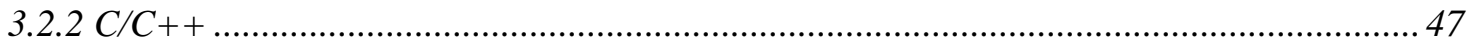

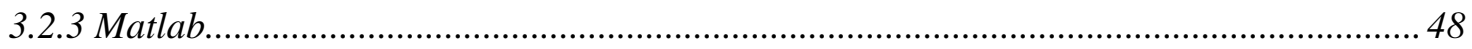

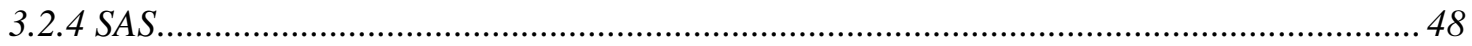

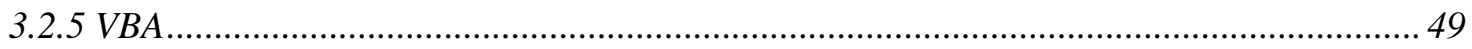

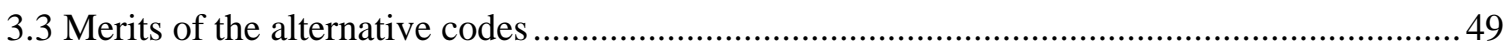

Chapter 4. The MSER Laboratory ........................................................................... 51 
Chapter 5. Implementation of MSER in Commercial Software ................................. 57

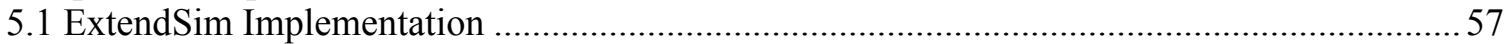

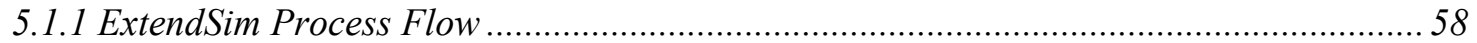

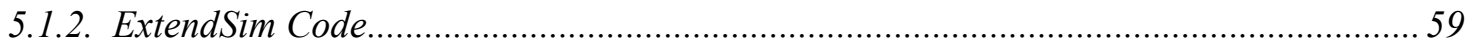

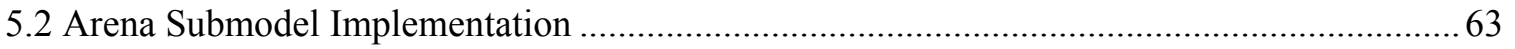

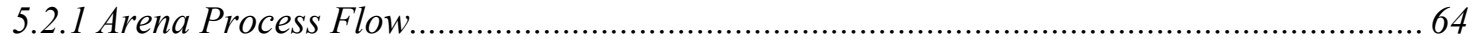

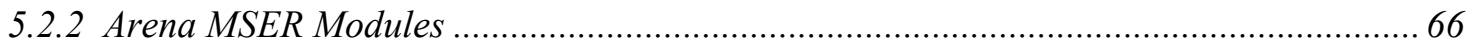

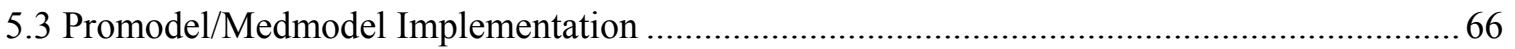

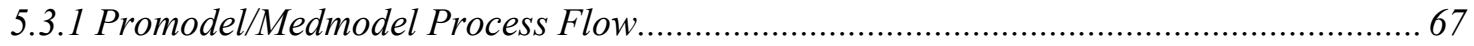

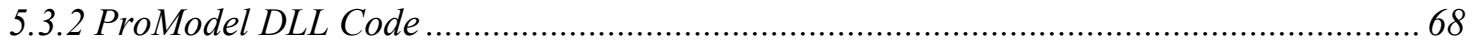

Chapter 6. Implementation in Post Analysis Codes ...................................................... 72

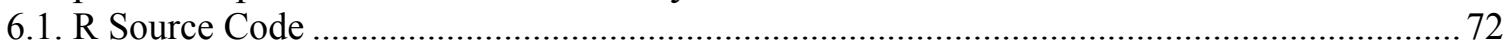

6.2. The SAS Source Code ............................................................................... 74

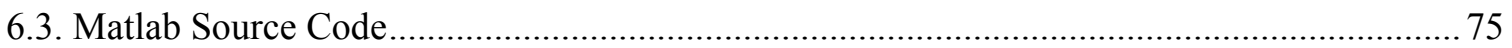

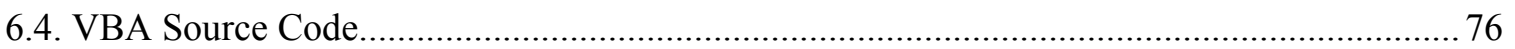

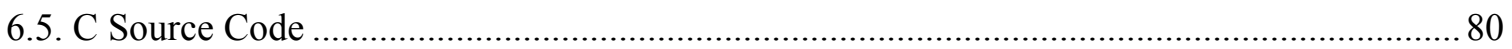

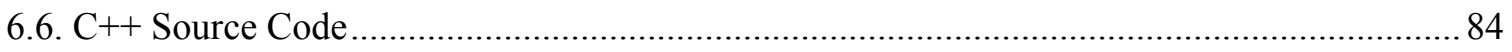

Chapter 7. Parameterization Issues, Analyses, and Results........................................ 90

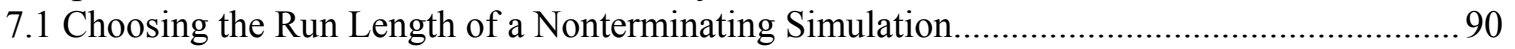

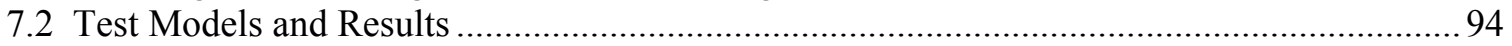

7.2.1 Model 1: Uniform Distribution with Superimposed Deterministic Bias ........................ 95

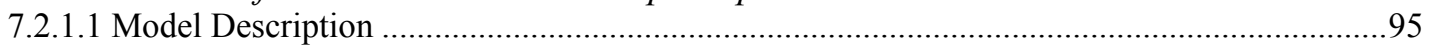

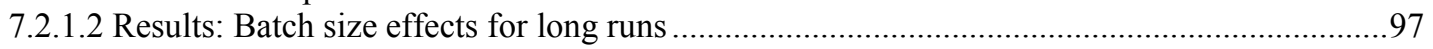

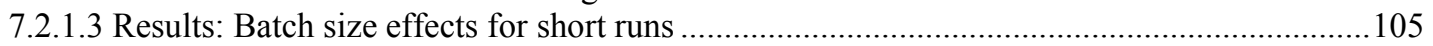

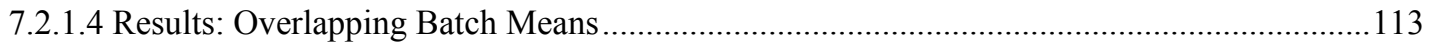

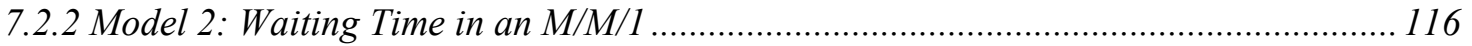

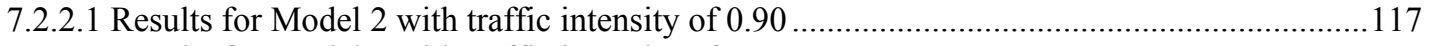

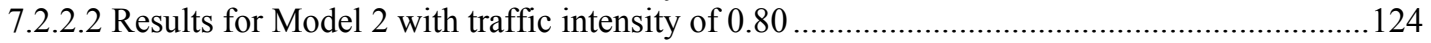

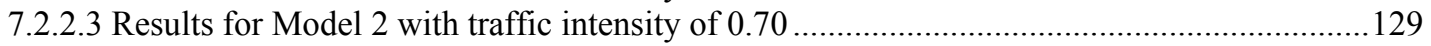

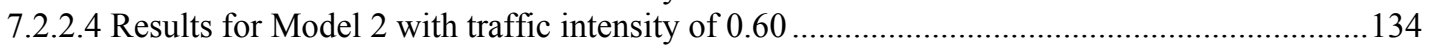

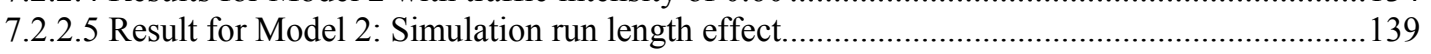

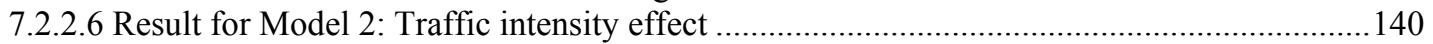

7.2.2.7 Results: Overlapping Batch Means for M/M/1 with Traffic Intensity of $0.90 \ldots \ldots \ldots \ldots \ldots \ldots \ldots \ldots . . . . .141$

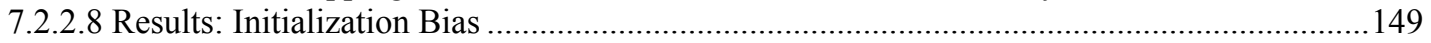

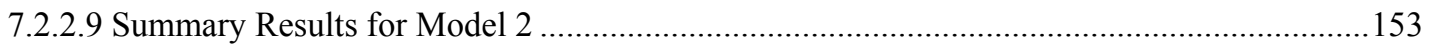

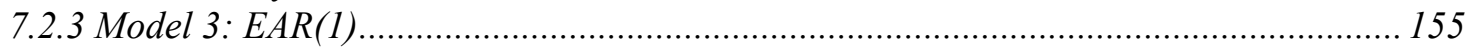

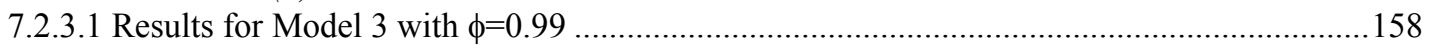

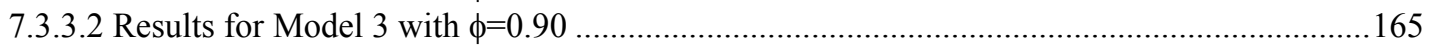

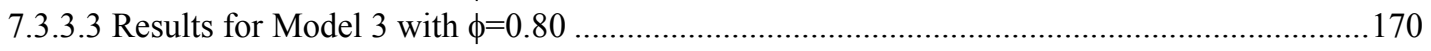

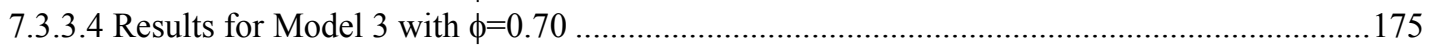

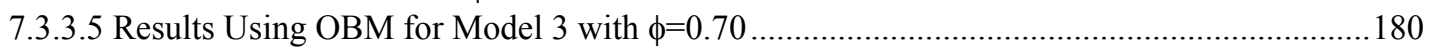

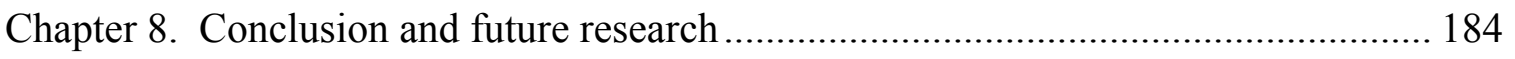

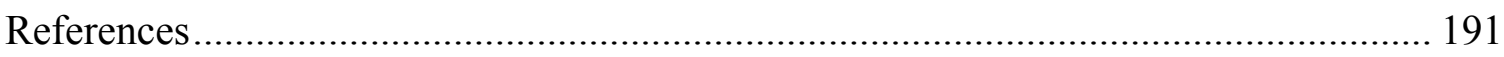

Appendix I. Arena MSER Submodel User Guide .................................................... 196

Appendix II. Personal reflections on the importance of the warm-up problem and

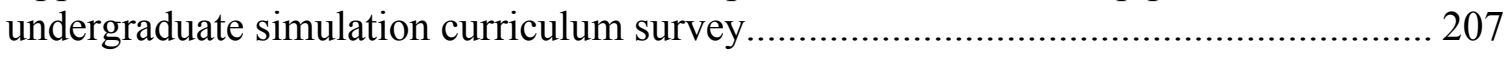

II.1 Anecdotal case to emphasize the importance of warm-up period ....................................... 207

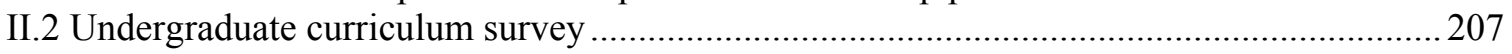




\section{LIST OF FIGURES}

Figure 1.1 Types of simulation (Law 2015) ............................................................... 3

Figure 1.2 Example of transient and steady-state density functions (after Law 2015)...... 6

Figure 2.1 Testing Module for identifying optimal truncation points in SIMUL8.......... 20

Figure 2.2 Output Analysis Tree for Wilson and Students ......................................... 24

Figure 3.1 Example of DLL usage in ProModel........................................................... 33

Figure 3.2 Specifying Warm-up Period in Arena's Run Setup Dialogue........................ 37

Figure 3.3 Warm-Up Period Determination in AutoMod................................................. 38

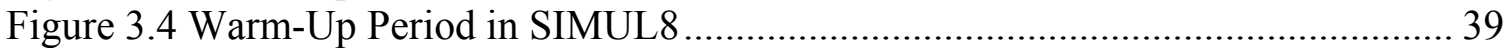

Figure 3.5 Output Analysis Support in SIMUL8 ......................................................... 39

Figure 3.6 Specifying a Warm-Up Period in ProModel ................................................ 40

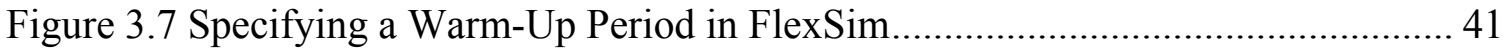

Figure 3.8 Specifying a Warm-Up Period in Simio....................................................... 42

Figure 3.9 Warm-Up Period in ExtendSim using Clear Statistics under Statistics library44

Figure 3.10 GUI of SimCAD ................................................................................... 45

Figure 4.1. Introduction of MSER Laboratory Web page .............................................. 53

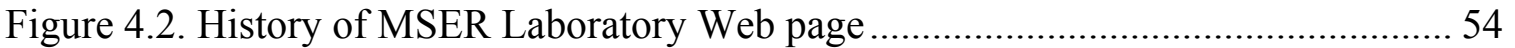

Figure 4.3. Sample Codes of MSER Laboratory Web page ......................................... 55

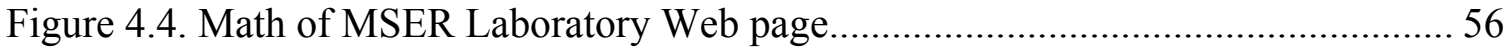

Figure 5.1 MSER Implementation and GUI in ExtendSim ......................................... 59

Figure 5.2 Main model of an M/M/1 queue in Arena with the MSER module included to

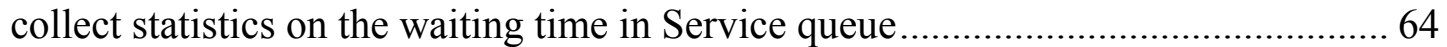

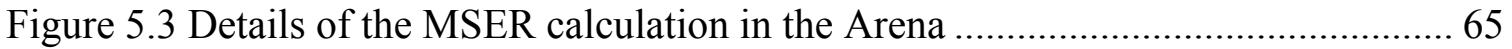

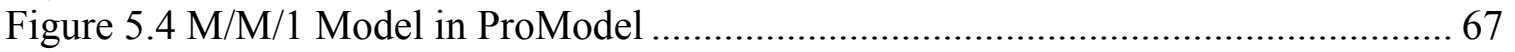

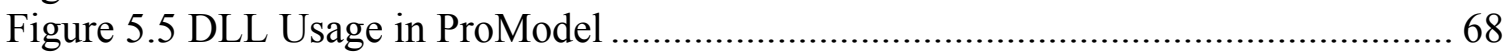

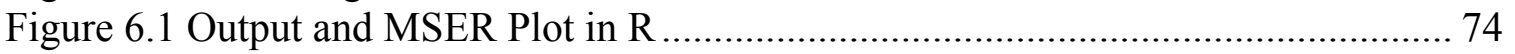

Figure 6.2 Output and MSER Plot in SAS ............................................................... 75

Figure 6.3 Output and MSER Plot in Matlab ................................................................ 76

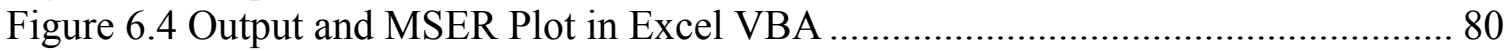

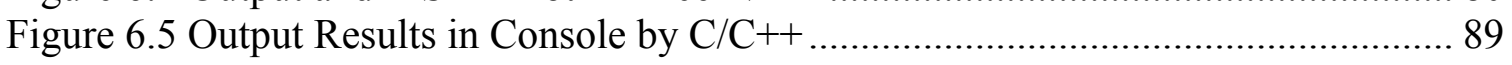

Figure 7.1 Hypothetical simulation output sequences illustrating one potential consequence

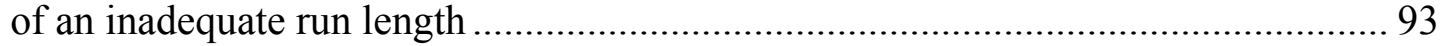

Figure 7.2 Hypothetical extensions of the simulation output sequences in Figure 7.1 illustrating further potential consequences of an inadequate run length .................... 94

Figure 7.3 Output for a representative replication of the first model ............................ 96

Figure $7.495 \%$ confidence intervals for (a) the truncated means for run lengths $n=10000$ and batch sizes $b=1,5,10$ and (b) the sample standard deviation in the estimated means

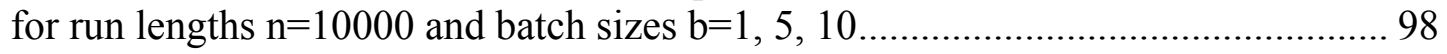


Figure 7.5 Fits to the steady-state sampling distributions of the mean for 1000 replications for run length $n=10,000$. Fits for all three batch sizes tested are nearly identical for batch sizes $b=1,5$, and 10 . 99

Figure $7.695 \%$ confidence intervals for (a) the MSER mean and (b) the standard deviation in the MSER truncation point for run lengths $n=10000$ and batch sizes $b=1,5,10 . .99$

Figure 7.7 Scatterplots of the truncated mean vs. the number of observations truncated for batch sizes $b=1,5$, and 10 for run length $n=10,000$.... 100

Figure 7.8 Frequency distribution of the total number of observations truncated as a function of batch size for batch sizes $b=1,5$, and 10 for run length $n=10,000 \ldots \ldots . .101$

Figure 7.9 Batched means and MSER statistic for the first 500 observations for replication 958 for batch sizes $b=1,5$, and 10 for run length $n=10,000$ 102

Figure 7.10 Batch means and MSER statistic for the first 1500 observations for replication 958 for batch sizes $b=10$ and run length $n=10,000$ 103

Figure 7.11 Estimated steady-state distribution (after truncation) for a single representative replication for run length $n=10,000$, with uniform fit for batch size $b=1$ and normal fits for batch sizes $b=5$ and 10 . 104

Figure 7.12 Scatterplots of the truncated mean vs. the number of observations truncated batch sizes $b=1,5$, and 10 for run lengths $n=175,200,500$, and 500 ..... 109

Figure 7.13 Frequency distribution of the total number of observations truncated as a function of batch size for batch sizes $b=1,5$, and 10 for run lengths of $n=175,200$, 500,500 ....

Figure $7.1495 \%$ confidence intervals of the mean for the truncated mean output as a function of batch size for batch sizes $b=1,5$, and 10 for run lengths of $n=175,200,300$, 500 .

Figure $7.1595 \%$ confidence intervals for the mean for the number of observations truncated for batch sizes $b=1,5$, and 10 for run lengths of $n=175,200,300,500 \ldots 112$

Figure 7.16 Representative Output, Overlapping Batch Mean, and MSER Statistic of Model $1(b=10,35,75$, and 150$)$..

Figure $7.1795 \%$ confidence intervals for Model 1 on (a) the truncated mean for overlapping and non-overlapping batches and (b) the standard deviation for overlapping and non-overlapping batches....................................................... 114

Figure 7.18 Scatterplots of the truncated mean vs. the number of observations truncated for OBM sizes $b=10,35,75$ and 150 for run length of $n=500$ for Model 1 (including NOBM batch size of 10) 115

Figure 7.19 Frequency distribution of the number of observations truncated as a function of OBM sizes $b=10,35,75$, and 150 for run length of $n=500$ for Model 1 (including NOBM batch size of 10) 115

Figure 7.20 Representative Output of Waiting-Time in an $\mathrm{M} / \mathrm{M} / 1$ with Traffic Intensity of $0.9\left(\mathrm{n}=64000\right.$; Blue line: $\left.\mathrm{E}\left(\mathrm{W}_{\mathrm{q}}\right)=81\right)$.

Figure $7.2195 \%$ confidence intervals for the mean and the truncated mean as a function of batch size $(b=1,5$, and 10$)$ and run length $(n=1000,2000,4000,8000,16000,32000$, and 64000) for Model 2 with traffic intensity of 0.9 (Theoretical mean of 81, Blue line)

Figure $7.2295 \%$ confidence intervals for the mean number of observations truncated as a function of batch size $(b=1,5$, and 10) and run length $(n=1000,2000,4000,8000$, 16000,32000 , and 64000) for Model 2 with traffic intensity of 0.9 . 120 
Figure 7.23 Scatterplots of the truncated mean vs. the number of observations truncated for batch sizes $b=1,5$, and 10 for run length of $n=1000,2000,4000,8000,16000,32000$, and 64000 for Model 2 with traffic intensity of 0.9 121

Figure 7.24 Frequency distribution of the number of observations truncated as a function of batch sizes $b=1,5$, and 10 for run length of $n=1000,2000,4000,8000,16000$, 32000, and 64000 for Model 2 with traffic intensity of 0.9

Figure 7.25 Example of M/M/1 with traffic intensity of $0.8(n=16000$, Blue line: $E(X))$ 124

Figure $7.2695 \%$ confidence intervals of the mean for the truncated mean output as a function of batch size $(b=1,5$, and 10) and run length $(n=1000,2000,4000,8000$, and 16,000) for Model 2 with traffic intensity of 0.80 (Theoretical mean of 32, Blue line)

124

Figure $7.2795 \%$ confidence intervals for the mean number of observations truncated as a function of batch size $(b=1,5$, and 10) and run length $(n=1000,2000,4000,8000$, and 16,000) for Model 2 with traffic intensity of 0.80 . 125

Figure 7.28 Scatterplots of the truncated mean vs. the number of observations truncated for batch sizes $b=1,5$, and 10 for run length of $n=1000,2000,4000,8000$, and 16,000 Model 2 with traffic intensity of 0.80 126

Figure 7.29 Frequency distribution of the number of observations truncated as a function of batch sizes $b=1,5$, and 10 for run length of $n=1000,2000,4000,8000$, and 16,000 for Model 2 with traffic intensity of 0.80 . 128

Figure 7.30 Example of M/M/1 with traffic intensity of $0.70(n=16000$, Blue line: $E(X))$ 129

Figure $7.3195 \%$ confidence intervals of the mean for the truncated mean output as a function of batch size $(b=1,5$, and 10) and run length $(n=1000,2000,4000,8000$, and 16,000 ) for Model 2 with traffic intensity of 0.70 (Theoretical mean of 16.33 , Blue line). 129

Figure 7.32 95\% confidence intervals for the mean number of observations truncated as a function of batch size $(b=1,5$, and 10) and run length $(n=1000,2000,4000,8000$, and 16,000) for Model 2 with traffic intensity of 0.70 ..... 130

Figure 7.33 Scatterplots of the truncated mean vs. the number of observations truncated for batch sizes $b=1,5$, and 10 for run length of $n=1000,2000,4000,8000$, and 16,000 Model 2 with traffic intensity of 0.7

Figure 7.34 Frequency distribution of the number of observations truncated as a function of batch sizes $b=1,5$, and 10 for run length of $n=1000,2000,4000,8000$, and 16,000 for Model 2 with traffic intensity of 0.70 . 133

Figure 7.35 Example of $M / M / 1$ with traffic intensity of $0.60(n=16000$, Blue line: $E(X))$ 134

Figure.7.36 95\% confidence intervals of the mean for the truncated mean output as a function of batch size $(b=1,5$, and 10) and run length $(n=1000,2000,4000,8000$, and 16,000) for Model 2 with traffic intensity of 0.60 (Theoretical mean of 9, Blue line)

Figure $7.3795 \%$ confidence intervals for the mean number of observations truncated as a function of batch size $(b=1,5$, and 10) and run length $(n=1000,2000,4000,8000$, and 16,000) for Model 2 with traffic intensity of 0.60 135 
Figure 7.38 Scatterplots of the truncated mean vs. the number of observations truncated for batch sizes $b=1,5$, and 10 for run length of $n=1000,2000,4000,8000$, and 16,000 Model 2 with traffic intensity of 0.60 136

Figure 7.39 Frequency distribution of the number of observations truncated as a function of batch sizes $b=1,5$, and 10 for run length of $n=1000,2000,4000,8000$, and 16,000 for Model 2 with traffic intensity of 0.60 . 138

Figure 7.40 Correlation between truncated means and observations truncated as a function of run length and traffic intensity 139

Figure 7.41 Correlation between truncated means and observations truncated by different traffic intensity (Run length of 16000). 140

Figure 7.42 Correlation between truncated means and observations truncated by different traffic intensity (Run length of 8000, 4000, 2000, and 100) ............................... 141

Figure 7.43 Representative Output, Overlapping Batch Mean, and MSER Statistic of Model 2 with traffic intensity of $0.90(b=10,50,100$, and 200)........................ 142

Figure $7.4495 \%$ confidence intervals for Model 3 on (a) the truncated mean for overlapping and non-overlapping batches and (b) the standard deviation for overlapping and non-overlapping batches......................................................... 143

Figure 7.45 Scatterplots of the truncated mean vs. the number of observations truncated for OBM sizes $b=10,50,100$ and 200 for run length of $n=64,000$ with traffic intensity of 0.90 for Model 2 (including NOBM batch size of 10). 144

Figure 7.46 Frequency distribution of the number of observations truncated as a function of OBM sizes $b=10,50,100$, and 200 for run length of $n=64,000$ with traffic intensity of 0.90 for Model 2 (including NOBM batch size of 10) 145

Figure $7.4795 \%$ confidence intervals for $\mathrm{M} / \mathrm{M} / 1$ with traffic intensity of 0.90 about the truncated mean for overlapping and non-overlapping batches $(n=1000,2000,4000$, $8000,16000,32000$, and 64000.).... 148

Figure 7.48 Output time series with traffic intensity of 0.90 with $b=1$ and $n=32,000149$

Figure 7.49 Confidence interval for Mean and Standard Deviation, Scatter Plot for Truncation points and Truncated Mean, Frequency Distribution of the Number of Observations Truncated with Traffic Intensity of 0.90 with $b=1,5$, and 10 and $n=$ 64,000 and 32,000 150

Figure 7.50 Representative output for $\operatorname{EAR(1)~for~run~length~} n=500$ and batch size $b=1$, illustrating the dependency of the steady-state mean and rate of convergence on the parameter for $\phi=\{0.7,0.8,0.9,0.99\}$ 156

Figure 7.51 Example of Model 3 with $\phi=0.99(n=16000$, Brown line: $E(X)) \ldots \ldots \ldots \ldots . . . .158$ Figure $7.5295 \%$ confidence intervals of the mean for the truncated mean output as a function of batch size $(b=1,5$, and 10$)$ and run length $(n=100,150,300,500,1000$, 2000, 4000, 8000, and 16,000) for Model 3 with $\phi=0.99$ (Theoretical mean of 100 , Blue line). 160

Figure $7.5395 \%$ confidence intervals for the mean number of observations truncated as a function of batch size $(b=1,5$, and 10$)$ and run length $(n=100,150,300,500,1000$, $2000,4000,8000$, and 16,000) for Model 3 with $\phi=0.99$ 161

Figure 7.54 Scatterplots of the truncated mean vs. the number of observations truncated for batch sizes $b=1,5$, and 10 for run length of $n=100,150,300,500,1000,2000,4000$, 8000 , and 16,000 Model 3 with $\phi=0.99$ 162 
Figure 7.55 Frequency distribution of the number of observations truncated as a function of batch sizes $b=1,5$, and 10 for run length of $n=100,150,300,500,1000,2000,4000$, 8000 , and 16,000 for Model 3 with of 0.99 .... 164

Figure 7.56 Example of EAR(1) with $\phi$ of $0.9(n=16000$, Blue line: $E(X)) \ldots \ldots \ldots \ldots . . .165$ Figure $7.5795 \%$ confidence intervals of the mean for the truncated mean output as a function of batch size $(b=1,5$, and 10) and run length $(n=100,150,300,500,1000$, 2000, 4000, 8000, and 16,000) for Model 3 with $\phi=0.90$ (Theoretical mean of 10 , Blue line)..... 165

Figure $7.5895 \%$ confidence intervals for the mean number of observations truncated as a function of batch size $(b=1,5$, and 10) and run length $(n=100,150,300,500,1000$, $2000,4000,8000$, and 16,000) for Model 3 with $\phi=0.90$ 166

Figure 7.59 Scatterplots of the truncated mean vs. the number of observations truncated for batch sizes $b=1,5$, and 10 for run length of $n=100,150,300,500,1000,2000,4000$, 8000 , and 16,000 Model 3 with $\phi=0.90$

Figure 7.60 Frequency distribution of the number of observations truncated as a function of batch sizes $b=1,5$, and 10 for run length of $n=100,150,300,500,1000,2000,4000$, 8000 , and 16,000 for Model 3 with $\phi$ of 0.90

Figure 7.61 Example of EAR(1) with $\phi$ of $0.8(n=16000$, Green line: $E(X)) \ldots \ldots \ldots \ldots . . .170$

Figure $7.6295 \%$ confidence intervals of the mean for the truncated mean output as a function of batch size $(b=1,5$, and 10) and run length $(n=100,150,300,500,1000$, $2000,4000,8000$, and 16,000) for Model 3 with $\phi=0.80$ (Theoretical mean of 5, Blue line) 170

Figure $7.6395 \%$ confidence intervals for the mean number of observations truncated as a function of batch size $(b=1,5$, and 10) and run length $(n=100,150,300,500,1000$, $2000,4000,8000$, and 16,000) for Model 3 with $\phi=0.80$ 171

Figure 7.64 Scatterplots of the truncated mean vs. the number of observations truncated for batch sizes $b=1,5$, and 10 for run length of $n=100,150,300,500,1000,2000,4000$, 8000 , and 16,000 Model 3 with $\phi=0.80$ 172

Figure 7.65 Frequency distribution of the number of observations truncated as a function of batch sizes $b=1,5$, and 10 for run length of $n=100,150,300,500,1000,2000,4000$, 8000 , and 16,000 for Model 3 with $\phi$ of 0.80 174

Figure 7.66 Example of EAR(1) with $\phi$ of $0.7(n=16000$, Blue line: $E(X)) \ldots \ldots \ldots \ldots . . . .175$ Figure $7.6795 \%$ confidence intervals of the mean for the truncated mean output as a function of batch size $(b=1,5$, and 10) and run length $(n=100,150,300,500,1000$, 2000, 4000, 8000, and 16,000) for Model 3 with $\phi=0.70$ (Theoretical mean of 3.33, Blue line)..... 175

Figure $7.6895 \%$ confidence intervals for the mean number of observations truncated as a function of batch size $(b=1,5$, and 10) and run length $(n=100,150,300,500,1000$, 2000, 4000, 8000, and 16,000) for Model 3 with $\phi=0.70$ 176

Figure 7.69 Scatterplots of the truncated mean vs. the number of observations truncated for batch sizes $b=1,5$, and 10 for run length of $n=100,150,300,500,1000,2000,4000$, 8000 , and 16,000 Model 3 with $\phi=0.70$

Figure 7.70 Frequency distribution of the number of observations truncated as a function of batch sizes $b=1,5$, and 10 for run length of $n=100,150,300,500,1000,2000,4000$, 8000 , and 16,000 for Model 3 with $\phi$ of 0.70 
Figure 7.71 Representative Output, Overlapping Batch Mean, and MSER Statistic of Model $3(b=10,50,100$, and 200). 180

Figure $7.7295 \%$ confidence intervals for Model 3 on (a) the truncated mean for overlapping and non-overlapping batches and (b) the standard deviation for overlapping and non-overlapping batches. 181

Figure 7.73 Scatterplots of the truncated mean vs. the number of observations truncated for OBM sizes $b=10,50,100$ and 200 for run length of $n=1000$ with $\phi$ of 0.70 for Model 3 (including NOBM batch size of 10). 182

Figure 7.74 Frequency distribution of the number of observations truncated as a function of OBM sizes $b=10,50,100$, and 200 for run length of $n=1000$ with $\phi$ of 0.70 for Model 3 (including NOBM batch size of 10) 182 


\section{LIST OF TABLES}

Table 2.1 Methods for Determining Start-up Periods (after Hoad et al. (2008))............. 18

Table 3.1 Properties of Warm-Up Period Control in Simio ........................................... 43

Table $7.195 \%$ confidence intervals for the mean and variance for the truncated mean output as a function of batch size for batch sizes $b=1,5$, and 10 for run length $n=10,000$

Table 7.2 . 95\% confidence intervals for the mean and variance for the number of observations truncated for batch sizes $b=1,5$, and 10 for run length $n=10,000 \ldots \ldots .99$

Table 7.3 Correlation between the truncated mean and the number of observations

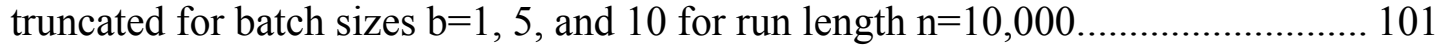

Table 7.4 Summary statistics for the steady-state data given in Figure 7.11 ................ 105

Table $7.595 \%$ confidence intervals for Model 1 on the truncated mean and the standard deviation for overlapping and non-overlapping batches

Table 7.6. Correlation between the truncated mean and the number of observations truncated for OBM sizes $b=10,35,75,150$ for run length of $n=500$ for Model 1 .. 116

Table 7.7. Theoretical Waiting Time in Queue by Traffic Intensity ............................ 117

Table 7.8 Correlation between the truncated mean and the number of observations truncated for batch sizes $b=1,5$, and 10 for run length of $n=1000,2000,4000,8000$, 16000,32000 , and 64000 for Model 2 with traffic intensity of 0.9 .

Table 7.9 Correlation between the truncated mean and the number of observations truncated for batch sizes $b=1,5$, and 10 for run length of $n=1000,2000,4000,8000$, and 16,000 for Model 2 with traffic intensity of $0.80 \ldots$

Table 7.10 Correlation between the truncated mean and the number of observations truncated for batch sizes $b=1,5$, and 10 for run length of $n=1000,2000,4000,8000$, and 16,000 for Model 2 with traffic intensity of 0.70 .

Table 7.11 Correlation between the truncated mean and the number of observations truncated for batch sizes $b=1,5$, and 10 for run length of $n=1000,2000,4000,8000$, and 16,000 for Model 2 with traffic intensity of 0.60

Table 7.12 95\% confidence intervals for Model 2 on the truncated mean and the standard deviation for overlapping and non-overlapping batches 143

Table 7.13 Correlation between the truncated mean and the number of observations truncated for OBM sizes $b=10,50,100$, and 200 for run length of $n=64,000$ for Model 2 .

Table7.14 Confidence Intervals for $\mathrm{M} / \mathrm{M} / 1$ with traffic intensity of 0.90 on the truncated mean and the standard deviation for overlapping and non-overlapping batches with traffic intensity of $0.90(\mathrm{n}=1000,2000,4000,8000,16000,32000$, and 64000) .. 146

Table 7.15 Correlation between the truncated mean and the number of observations truncated for $b=1,5$, and 10 for run length of $n=32,000$ with initial bias of $100 \ldots 151$

Table 7.16 95\% confidence intervals for Model 2 on the observation truncated and the standard deviation for run length of $n=32,000$ with initial bias of 100 .

Table $7.1795 \%$ confidence intervals for Model 2 on the truncated mean and the standard deviation for run length of $n=32,000$ with initial bias of 100 
Table7.18 95\% confidence intervals for Model 2 on the mean without truncation and the standard deviation for run length of $n=32,000$ with initial bias of 100 . 152

Table 7.19 Correlation between the truncated mean and the number of observations truncated for $b=1,5$, and 10 for run length of $n=64,000$ with initial bias of $100 \ldots 152$

Table 7.20 95\% confidence intervals for Model 2 on the observation truncated and the standard deviation for run length of $n=64,000$ with initial bias of 100 . 152

Table 7.21 95\% confidence intervals for Model 2 on the truncated mean and the standard deviation for run length of $n=64,000$ with initial bias of 100 152

Table 7.22 95\% confidence intervals for Model 2 on the mean without truncation and the standard deviation for run length of $n=64,000$ with initial bias of $100 \ldots \ldots \ldots \ldots \ldots \ldots . . . . . . .153$

Table 7.23 Expected Value of the Steady-State Mean as a function of $\phi . . . . . . . . . . . . . . . . . . . .155$

Table 7.24 Expected $\gamma$-percent settling time $\tau$ as a function of $\phi$ and $\gamma$ 157

Table 7.25 Correlation between the truncated mean and the number of observations truncated for batch sizes $b=1,5$, and 10 for run length of $n=100,150,300,500,1000$, $2000,4000,8000$, and 16,000 for Model 3 with $\phi=0.99$ 163

Table 7.26 Correlation between the truncated mean and the number of observations truncated for batch sizes $b=1,5$, and 10 for run length of $n=100,150,300,500,1000$, 2000, 4000, 8000, and 16,000 for Model 3 with $\phi=0.90$. 168

Table 7.27 Correlation between the truncated mean and the number of observations truncated for batch sizes $b=1,5$, and 10 for run length of $n=100,150,300,500,1000$, 2000, 4000, 8000, and 16,000 for Model 3 with $\phi=0.80$.

Table 7.28 Correlation between the truncated mean and the number of observations truncated for batch sizes $b=1,5$, and 10 for run length of $n=100,150,300,500,1000$, 2000, 4000, 8000, and 16,000 for Model 3 with $\phi=0.70$. 178

Table 7.29 95\% confidence intervals for Model 3 on the truncated mean and the standard deviation for overlapping and non-overlapping batches 181

Table 7.30 Correlation between the truncated mean and the number of observations truncated for OBM sizes $b=10,50,100$, and 200 for run length of $n=16,000$ with $\phi$ of 0.70 for Model 3 183

Table AII.1 Twenty Undergraduate Curricula of Simulation 209 


\section{Acknowledgement}

The journey of my $\mathrm{PhD}$ study is finally heading towards the last train station. However, this won't be the end of my learning process but a start of a new journey. I believe my one small step into the world of simulation is trivial, yet very meaningful for it will enlighten a person to be like me and have courage to work hard with intelligent people at University of Virginia.

I admit that my advisor, Prof. White Jr., is my first and final beacon in staying in the U.S. Without his considerate support and help, I wouldn't have come this far with only my contribution in discrete event simulation. In addition, I won't forget all the constructive and encouraging comments and guidance from other committee members for my life at the University of Virginia.

I would like to express my most sincere gratitude to my wife, Eun Hye Kwon, who has fully supported and believed me both mentally and emotionally throughout this journey. I also thank my son, Edward and daughter, Claire for understanding a busy father as well as my parents and my parents-in-law encouraging my unexpected long journey. 


\section{Abstract}

It is well known that the Mean Squared Error Rule (MSER) is an efficient and effective method for mitigating initialization bias in the output analysis of steady-state, discreteevent simulation. However, the application of this method in research and practice has been delayed or misunderstood even by experienced simulation modelers. To address this issue, we develop the MSER Laboratory - a permanent website that provides user-friendly sample codes, as well as information needed to apply MSER intelligently. MSER modules for three commercial software packages, and standalone MSER codes in five popular programming languages, have been written, validated, and made publically available via the Laboratory.

In addition, we use these codes to address open issues in the selection of the parameters needed to apply MSER. These issues include the selection of the MSER truncation threshold, batch size, and batching scheme (overlapping or non-overlapping batch means), in conjunction with the determination of an initial run length for simulation replications. Experiments are conducted using three test models that pose differing challenges for the successful determination of a warm-up period. We confirm that, given adequate run lengths, MSER is both effective and robust in all cases. We also illustrate various consequences of foreshortened replications for each of the three models. 


\section{Executive Summary}

This research addresses a practical shortcoming in the output analysis of non-terminating, stochastic, discrete-event simulations (DES). Specifically, our concern is the application of MSER, an algorithm for determining an optimal warm-up period when estimating the steady-state mean of an output based on a sequence of simulated output values. It is noteworthy that MSER:

- is proven to yield a near-optimal estimate (under mild assumptions) in the sense of minimum mean-squared error (MSE) that cannot be improved upon a priori,

- is widely accepted in the academic literature as the preferred approach to mitigating bias associated with the arbitrary specification of initial conditions,

- is presented in detail and recommended in the current editions of many standard texts on DES, and

- is effective, efficient, robust, and intuitive.

In spite of these considerable merits, the application of MSER in practice appears far from universal. We speculate the unaided application of MSER can be inconvenient and potentially consuming of both analyst and computing time, especially when a large number of output sequences must be initialized. An obvious solution is to imbed MSER in an automated, dynamic, run-time procedure that requires minimal analyst interaction. 
However, the only reported effort to build MSER into a commercial simulation suite (SIMUL8) led to the suggestion that there are significant barriers to implementation (Hoad and Robinson, 2011). These include:

- the selection of run length,

- sequential data collection from multiple replications,

- output types associated with cumulative values and extrema, and

- data associated with entities.

Conventionally, MSER has been implemented as a data-driven postprocessor and, as such, requires that an output sequence be simulated before application. There is no guarantee that MSER will converge if the run length for this sequence is insufficient to capture a useful trailing segment of steady-state behavior, even for a stable system. Determination of an optimal warm-up period therefore is, in fact, confounded with problem of determining an adequate run length, which is most often resolved only by trial and error.

In this research we demonstrate that in application MSER typically will flag instances in which the run length is inadequate by truncating all (or at least a very large fraction) of the output sequence to which it is applied. However, we further demonstrate that there are pathological instances for which this is not the case. While Hoad et al. (2008) provide useful guidance, determining an appropriate run length a priori remains an open and perhaps intractable problem.

With this caveat, we demonstrate both theoretically and by application that the remaining barriers are readily overcome. Specifically, we:

- cast estimation of an output mean as an iterative optimization problem from which we derive the memory requirements for run-time implementation, 
- develop runtime versions of MSER for ExtendSim (as a static library), Arena (as a submodel), Promodel (as a DLL),

- develop MSER postprocessing codes in several popular programming languages, including the open-source languages $\mathrm{R}$ and $\mathrm{C} / \mathrm{C}++$, as well as the proprietary languages Matlab, SAS, and VBA, and

- create a prototype MSER Laboratory - a website to facilitate the distribution of MSER codes and supporting research online available at http://faculty.virginia.edu/MSER/.

MSER automation, the distribution of codes, and the creation of the Lab are principal contributions of this research.

Additionally, during the course of this research we encountered multiple instances of a perhaps obvious, but seemingly pervasive misconception regarding the application and evaluation initialization procedures related to MSER. At least two alternative approaches appear in the literature. The first applies MSER to individual output sequences, truncates each sequence accordingly, calculates the truncated mean for each sequence, and then averages the truncated means with weighting to estimate the steady-state mean. The second determines the output sequences for multiple replications, averages these sequences, applies MSER to the average sequence to determine a single warm-up period, and then estimates the steady-state mean based on the average sequence truncated by this period.

The first approach is preferred. MSER determines the optimal truncation point for the specific output sequence to which it is applied and will return an optimal estimate of the mean for each sequence when this exists. The second approach is almost certainly 
suboptimal. There is no reason to believe that the truncation point for the average sequence is optimal for each of the individual runs. The aggregate result is over-truncation for some of the sequences and under-truncation for the remainder.

The second approach has led to misgivings regarding the efficacy of MSER. These doubts surfaced most notably in Law (2015), who compares the average of the sample of individual truncation points with a theoretical mean truncation point. He erroneously concludes that MSER may not truncate an appropriately large number of observations. Wang and Glynn (2014) offer an argument that is similarly flawed.

A further contribution of this research is to highlight and correct this misconception with a set of three simple examples: (1) the response of a uniform white-noise process in steady-state with a superimposed linearly-decreasing deterministic transient, (2) the delay times in an M/M/1 queue, and (3) the response of an EAR(1) process. For these test cases we show that, given adequate run lengths, the MSER estimate of the steady-state mean is uncorrelated with the MSER-optimal truncation point and therefore the success of a truncation procedure in terms of the accuracy of the estimate cannot be imputed from the truncation point alone. Indeed, we show that even modest correlation is a symptom of inadequate run lengths. We reiterate that the purpose of truncation is to determine the warm-up period that yields the most accurate and precise estimate of the steady-state mean. Other proposed measures of performance are at best irrelevant and at worse seriously misleading.

We use these same examples to explore the sensitivity of the estimated mean to run length $n$ and to the choice of the MSER parameters $b$ (batch size) and $d_{\max }$ (the maximum acceptable optimal truncation point on the range of a given run length $\left.\left[0 \leq d_{\max } \leq n\right]\right)$. We 
show that longer run lengths and smaller batch sizes in general better serve to find acceptable truncation points in all models. For all experiments with sufficient runs lengths and small batches, however, MSER is consistently effective in yielding near-optimal truncation points, irrespective of the character of the response. For models with strong negative or positive trends in the transient sequences, such as Models 1 and 3, MSER remains highly effective even with relatively short runs for which $d_{\max }$ is greater than $n / 2$, a maximum threshold suggested in the literature. This is especially true for models in which there is a sharp transition from the transient to the steady-state operating regimes, such as Model 1.

Models that are characterized by oscillatory responses, including such regenerative processes such as queues, represent the greatest challenge for MSER among the three test cases. In particular, the specification of $d_{\max }$ becomes an issue. For long runs, the proportion of runs that violate the $d_{\max } \leq n / 2$ threshold is comparatively small and the truncated mean and the truncation point are independent. MSER has ample data representative of steady-state with which to work.

As $n$ decreases, this is no longer the case. The number of violations increases dramatically and the correlation becomes increasingly negative, even becoming significant at $\alpha=0.3$ in the most extreme cases. This implies that smaller (biased) mean estimates are associated with the greater truncation. Since the average error in the estimates seems always to be negative when starting queuing systems from empty and idle, violations of the threshold cause greater estimation error. Enforcing the stringent rule of $n / 2$ improves the estimates dramatically. 
Batching rarely improves MSER performance and, as might be guessed, is contraindicated for short runs. We speculate (but do not attempt to confirm) that the superior performance of MSER-5 reported in the literature is a consequence of the central limit theorem and results from normalizing the geometric sampling distribution of mean number in system for an $\mathrm{M} / \mathrm{M} / 1$ queue, with the effect of improving coverage.

The batching scheme conventionally applied in MSER- $b$ uses non-overlapping batches each with batch size $b$. We also explored the application of overlapping batch means (OBM) as an alternative, as suggested by Pasupathy and Schmeiser (2010), for a range of alternative batch sizes. We find that with mild noise amplitudes, OBM tends to outperform NOBM. However, the performance of OBM appears to be more sensitive to batch size and simulation run lengths in comparison to NOBM.

This dissertation is organized as follows:

- Chapter 1: Introduction

- Chapter 2: Literature review

- Chapter 3: MSER Implementation Issues

- Chapter 4: MSER Laboratory

- Chapter 5: MSER implementation in commercial languages

- Chapter 6: MSER implementation in Post-Analysis Codes

- Chapter 7: Parameterization Issues, Analyses, and Results

- Chapter 8: Conclusion and future research

- References

- Appendix I: Arena MSER Submodel User's Guide 
- Appendix II: Personal reflections on the importance of the warm-up problem and undergraduate simulation curriculum survey 


\section{Chapter 1: Introduction}

This research addresses a practical shortcoming in the output analysis of nonterminating, stochastic, discrete-event simulations (DES). Specifically, our concern is the application of MSER, an algorithm for determining an optimal warm-up period when estimating the steady-state mean of an output based on a sequence of simulated output values. In this chapter we briefly review the importance of stochastic simulation, the types of simulation with respect to output analysis, the analysis of terminating and nonterminating simulations, introduce the warm-up problem, and outline the research issues and contributions.

\subsection{The Importance and Ubiquity of Discrete-Event, Stochastic Simulation}

Stochastic simulation has emerged as a critical tool for analysis, especially for complex systems that reflect current sophisticated and interacting real-time technologies. While developments in information technology and computer science promoted the use of simulation in various fields of academia and industry, remarkable advances computing power reduced the computational burdens in terms of both time and money. Students, engineers, analysts, practitioners and decision-makers are more and more dependent upon simulation because analytical solutions are rarely available for the design or implementation of complex systems. 
Simulation is widely applied in engineering, health, management, manufacturing, service industries, public systems, and almost all systems imaginable (Fishman, 2001). While simulation may be regarded as computational programming to replicate and imitate a real system with a reasonable model based on succinct assumptions, stochastic simulation more broadly is a computational sampling experiment and should be supported by sound statistical notions (Law 2015). Statistical output analysis provides the foundation needed to verify and validate the model simulating the system of interest.

\subsection{Types of Simulation with Respect to Output Analysis}

With respect to output analysis, simulations largely can be categorized into two groups, as shown in Figure 1.1: (1) terminating (or finite horizon) simulations and (2) nonterminating (or infinite horizon) simulations. For terminating simulations, initial and terminating run conditions are usually known (at least approximately) so that initial transients are a part of the natural behavior under investigation. In contrast, for nonterminating simulations, neither initial nor terminating conditions are specified and these must be invented for analysis. Serial correlation in output observations can lead to significant bias in performance estimators with a "poor" selection of initial conditions. Understanding and mitigating such biases is essential. 


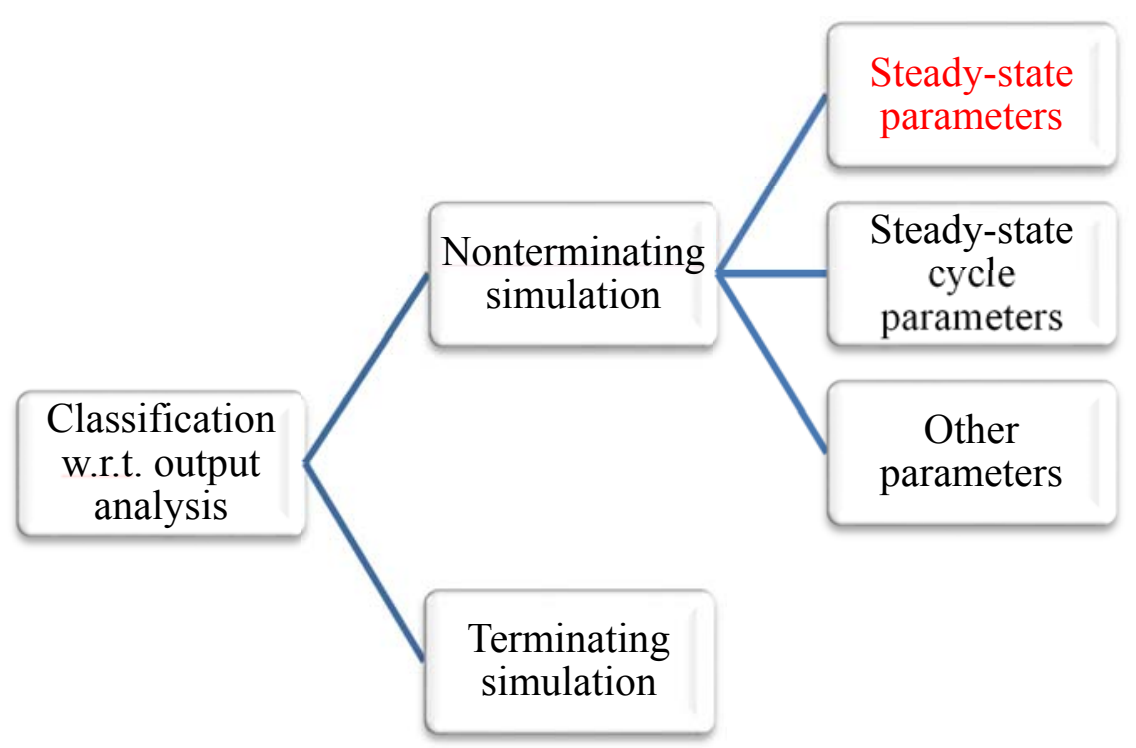

Figure 1.1 Types of simulation (Law 2015)

\subsection{The Analysis of Terminating Simulation Outputs}

It is useful to think of a simulation as the transformation of input random variables $U$ into output random variables $X$, s: $U \rightarrow X$. The output of a simulation replication is a sequence of observations, the (indexed) numbers $\left\{x_{i}, i=1,2, \ldots, n\right\}$. This sequence is a realization of the time series $\left\{X_{\mathrm{i}}, i=1,2, \ldots, n\right\}$, where the distribution of each of the output random variables is given by $F_{i}\left(X_{\mathrm{i}} \mid x_{0}\right)=\operatorname{Pr}\left(X_{\mathrm{i}} \leq x_{\mathrm{i}} \mid x_{0}\right)$ and $x_{0}$ is the initial condition.

The basic assumptions of standard statistics are that observations are i.i.d. (independent and identically distributed) and normally distributed (or that $n$ is sufficiently large to invoke the central limit theorem). In other words, all of the observations are drawn from the same normal distribution

$$
F_{i}\left(X_{i} \mid x_{0}\right)=F\left(X_{\mathrm{i}} \mid x_{0}\right) \forall i=1,2, \ldots, n
$$


These assumptions are not met by observations within the series. First, the observations typically are sequentially correlated and therefore not independent. Second, the transient distributions $F_{i}\left(X_{\mathrm{i}}, x_{0}\right)$ typically are different for each observation index $i$ and therefore the observations are not identically distributed. Third, there is no guarantee that these distributions are normal.

For terminating simulations, this difficulty is easily overcome. Each replication, $j=1,2, \ldots, N$, yields one observation of the statistic of interest $Y_{j}$, such as the sample mean

$$
Y_{j}=\bar{X}_{j}=\frac{1}{n} \sum_{i=1}^{n} X_{i}
$$

Running $N$ independent replications of the simulation yields a set observations drawn from the sampling distribution for this statistic, $\left\{Y_{\mathrm{i}}, i=1,2, \ldots, N\right\}$. These observations are i.i.d. and therefore the mean across replications

$$
\bar{Y}=\frac{1}{N} \sum_{j=1}^{N} Y_{j}
$$

is an unbiased estimator for the output statistic

$$
\lim _{N \rightarrow \infty} \bar{Y}=E[X]=\mu_{X}
$$

Moreover, because the sampling distribution of $Y_{j}$ is approximately normal (by the central limit theorem for sufficiently large $N$ ), the precision of the estimate of $\mu_{X}$ can be estimated as confidence interval by the standard formula

$$
\bar{Y} \pm t_{N-1,1-\alpha / 2} \sqrt{\frac{S_{N}^{2}}{N}}
$$

where the sample variance of $Y_{j}$ is an unbiased estimator for the variance of $Y_{j}$ 


$$
\lim _{N \rightarrow \infty} S_{N}^{2}=\sigma_{Y}
$$

Thus the basic assumptions of standard statistics are satisfied for summary statistics across replications.

\subsection{The Analysis of Nonterminating Simulation Outputs}

The approach described above works because the transient response is the object of our analysis for terminating simulations. This is not the case for nonterminating systems, however, and performing independent replications alone is inadequate. For nonterminating simulations, all of the observations within each replication must be drawn from the steadystate distribution $F(X)=\operatorname{Pr}(X \leq x)$ otherwise the estimator for the statistic is biased. Because $F(X)$ is not independent of the initial conditions at the beginning, the difficulty posed is variously referred to as the problem of the initial transient, the start-up problem, or the warm-up problem.

While there are many proposed alternatives, the most common approach to resolving the warm-up problem is based on the idea that the transient distributions converge to the steady-state distribution as the index gets large

$$
F\left(X_{\mathrm{i}} \mid x_{0}\right) \rightarrow F(X) \text { as } i \rightarrow \infty
$$

As suggested in Figure 1.2, after some number of observations $d$, the transient distribution is sufficiently close to the steady-state distribution to mitigate bias in the output statistic, i.e.,

$$
F\left(X_{\mathrm{i}} \mid x_{0}\right) \approx F(X) \forall i \geq d+1 \text {. }
$$




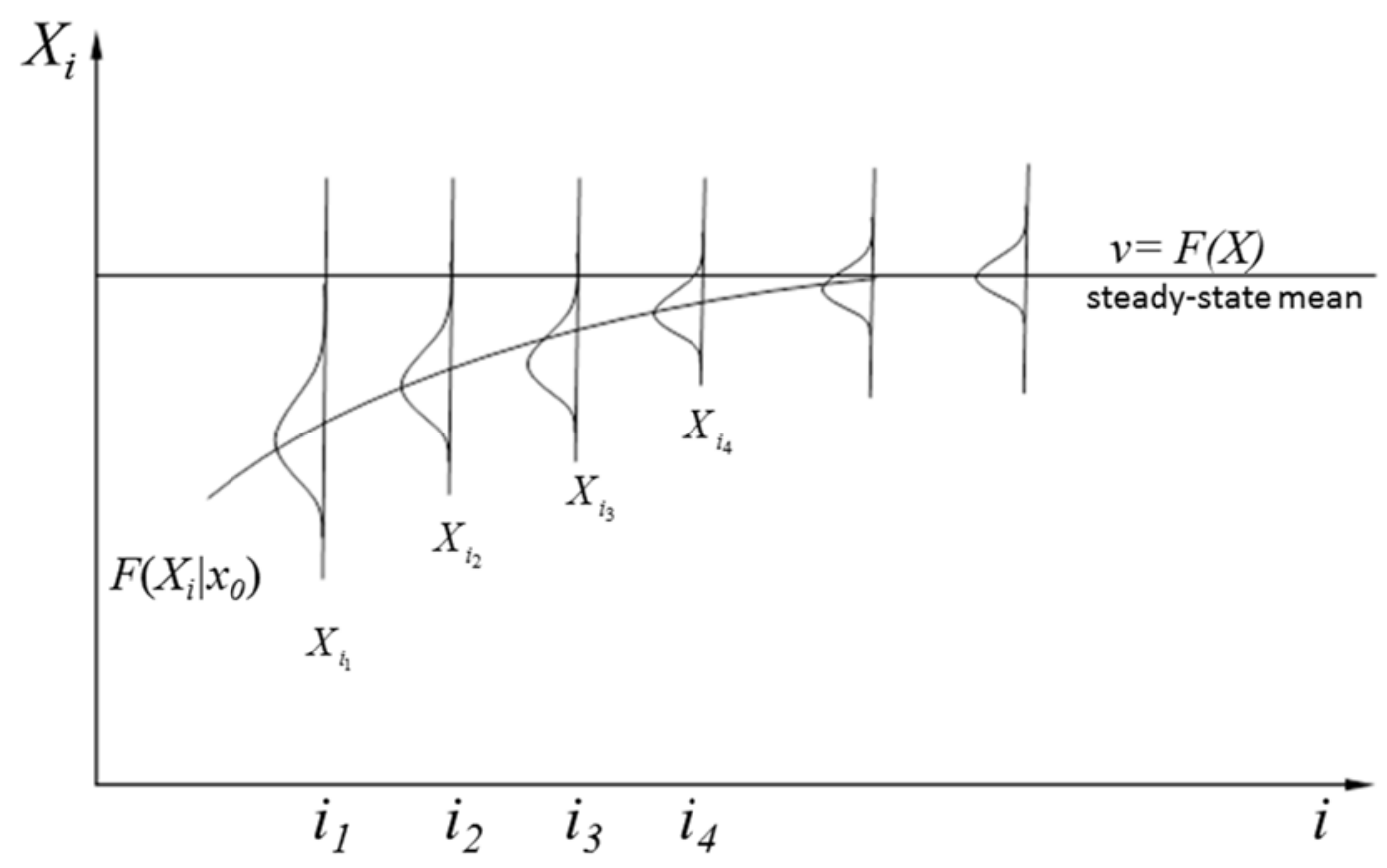

Figure 1.2 Example of transient and steady-state density functions (after Law 2015)

By allowing the simulation to "warm up"--discarding all observations prior to $X_{d+1}$ within the output series when computing the statistic - the problem can be overcome. On each replication we observe the truncated sample mean

$$
Y_{j, d}=\bar{X}_{j, d}=\frac{1}{n-d} \sum_{i=d+1}^{n} X_{i}
$$

The warm-up problem then reduces to that of determining the best truncation point $d$ for each replication $j$. As a matter of convenience, very often a single, conservatively large value of $d$ is selected and used for all replications.

It should be noted that the requirement for convergence in distribution can be relaxed in some cases. If our interest is estimating the steady-state mean, for example, it is sufficient that the process is covariance stationary, i.e., that 
(1) the mean exists $\mu=E\left[X_{i}\right]<\infty \forall i=1,2, \ldots, n$

(2) the variance exists $\sigma^{2}=\operatorname{Var}\left[X_{i}\right]<\infty \forall i=1,2, \ldots, n$, and

(3) the autocovariance function of order $r$

$$
R(r)=\operatorname{cov}\left(X_{i}, X_{i-r}\right) \forall k, 0 \leq r \leq n-1
$$

is not a function of $i$.

In other words, the means and variances of all observations are constant and the correlation between any two observations in the series depends only on the number of intervening observations and not on the location of these points in the series.

For a covariance stationary process, it can be shown that the variance of the sample mean (within-run) is

$$
\sigma^{2}[\bar{X}(n)]=\left[R(0)+2 \cdot \sum_{k=1}^{n-1}\left(1-\frac{k}{n}\right) R(k)\right] / n
$$

where $R(r)$ is the order $r$ autocovariance function defined above (Pawlikowski, 1990). For uncorrelated observations, $R(r)=0$ for all lags $r>0$, and therefore the variance reduces to the standard formula for i.i.d. observations

$$
\hat{\sigma}^{2}[\bar{X}(n)]=\sum_{i=1}^{n}\left\{x_{i}-\bar{X}(n)\right\}^{2} / n(n-1)
$$

From this we can see that if the correlation is strong and positive, ignoring it will lead to serious underestimation of the true variation. 


\subsection{The Locus of this Research}

As will be discussed in Chapter 2, many alternative approaches to determining $d$ have been proposed. Until the introduction of the MSER algorithm (White and Minnox, 1994), mitigating initialization bias in the mean was considered an open problem. All of the prior approaches where found wanting for various reasons. Over the past two decades, however, increasingly MSER has been accepted as a solution to the warm-up problem.

It is noteworthy that MSER:

- is proven to yield a near-optimal estimate (under mild assumptions) in the sense of minimum mean-squared error (MSE) that cannot be improved upon a priori,

- is widely accepted in the academic literature as the preferred approach to mitigating bias associated with the arbitrary specification of initial conditions,

- is presented in detail and recommended in the current editions of many standard texts on DES, and

- $\quad$ is effective, efficient, robust, and intuitive.

In spite of these considerable merits, the application of MSER in practice appears far from universal. We speculate the unaided application of MSER can be inconvenient and potentially consuming of both analyst and computing time, especially when a large number of output sequences must be initialized. An obvious solution is to imbed MSER in an automated, dynamic, run-time procedure that requires minimal analyst interaction.

However, the only reported effort to build MSER into a commercial simulation suite (SIMUL8) led to the suggestion that there are significant barriers to implementation (Hoad and Robinson, 2011). These include:

- the selection of run length, 
- sequential data collection from multiple replications,

- output types associated with cumulative values and extrema, and

- data associated with entities.

Conventionally, MSER is a data-driven postprocessor and, as such, requires that an output sequence be simulated before application. There is no guarantee that MSER will converge if the run length for this sequence is insufficient to capture a useful trailing segment of steady-state behavior. Determination of an optimal warm-up period therefore is in fact confounded with problem of determining an adequate run length, which is most often resolved by trial and error.

In this research we demonstrate that in application MSER typically will flag instances in which the run length is inadequate by truncating all (or at least a very large fraction) of the output sequence to which it is applied. However, we further demonstrate that there are pathological instances for which this is not the case. While Hoad et al. (2008) provide useful guidance, determining an appropriate run length a priori remains an open and perhaps intractable problem.

With this caveat, we demonstrate both analytically and by application that the remaining barriers are readily overcome. Specifically, we:

- cast estimation of an output mean as an iterative optimization problem from which we derive the memory requirements for run-time implementation (see the literature review in Chapter 2),

- describe and resolve MSER implementation issues (Chapter 3) 
- create a prototype MSER Laboratory (Chapter 4) - a website to facilitate the distribution of MSER codes and supporting research online (available at http://faculty.virginia.edu/MSER/).

- develop runtime versions of MSER in an ExtendSim static library, a Promodel DLL, and an Arena submodel (Chapter 5), and

- develop MSER post-processing codes in several popular programming languages, including the open-source languages $\mathrm{R}$ and $\mathrm{C} / \mathrm{C}++$, as well as the proprietary languages Matlab, SAS, and VBA (Chapter 6).

Additionally, during the course of this research we encountered multiple instances of a perhaps obvious, but seemingly pervasive, misconception regarding the application and evaluation initialization procedures such as MSER. At least two alternative approaches appear in the literature. The first applies MSER to individual output sequences, truncates each sequence accordingly, calculates the truncated mean for each sequence, and then averages the weighted truncated means to estimate the steady-state mean. The second determines the output sequences for multiple replications, averages these sequences, applies MSER to the average sequence to determine a single warm-up period, and then estimates the steady-state mean based on the average sequence truncated by this period.

The first approach is preferred. MSER determines the optimal truncation point for the specific output sequence to which it is applied and will return an optimal estimate of the mean for each sequence. The second approach is almost certainly suboptimal. There is no reason to believe that the truncation point for the average sequence is optimal for each of the individual runs. The aggregate result is over-truncation for some of the sequences and under-truncation for the remainder. A proof is provided. 
The second approach has led to misgivings regarding the efficacy of MSER. These doubts surfaced most notably in Law (2015), who compares the average of the sample of individual truncation points with a theoretical mean average truncation point. $\mathrm{He}$ erroneously concludes that MSER may not truncate an appropriately large number of observations. Wang and Glynn (2014) offer an argument similarly flawed.

A further contribution of this research (see Chapter 7) is to highlight and correct his misconception with a set of three simple examples: (1) the response of a uniform whitenoise process in steady-state with a superimposed linearly-decreasing deterministic transient, (2) the delay times in an $\mathrm{M} / \mathrm{M} / 1$ queue, and (3) the response of an $\operatorname{EAR}(1)$ process. For these test cases, we show that the MSER estimate of the steady state mean is uncorrelated with the MSER-optimal truncation point and therefore the success of a truncation procedure in terms of the accuracy of the estimate cannot be imputed from the truncation point alone. We reiterate that the purpose of truncation is to determine the warm-up period that yields the most accurate and precise estimate of the steady-state mean. Other proposed measures of performance are at best irrelevant and at worse seriously misleading. Also in Chapter 7 we use these same examples to explore the sensitivity of the estimate mean to run length $n$ and to the choice of the MSER parameters $b$ (batch size) and $d_{\max }$ (the maximum acceptable optimal truncation point on the range of a given run length $\left.\left[0 \leq d_{\max } \leq n\right]\right)$.

The final Chapter discusses the conclusions of this effort, together with potentially useful directions for further research. A User's Guide for application of the Arena submodel is provided in the Appendix I. Appendix II includes an anecdotal case with some 
personal reflections on the simulation enterprise and the importance of the warm-up problem, as well as a survey of undergraduate simulation curricula.

\subsection{Background: A Note on Smart Initialization}

One of the strengths of MSER is that it demands only that an optimal truncation point exists for a simulated model (i.e., stationary or weak convergence). In the past, some researchers have suggested that truncating or deleting an initial data series is not the best way to improve the estimate with respect to mean squared error (MSE). Blomqvist (1970), Wilson and Pritsker (1978), Turnquist and Sussman (1977), and Grassmann (2009, 2011) instead advocated the "smart" choice of an initial condition to mitigate biases and generate a robust result.

However, it is rather difficult to search for an optimal starting point unless the characteristics of simulation are known a priori. Do we still need to recognize the existence of initialization bias? We firmly believe that the answer should be "yes". We will be better off by presuming almost every probabilistic non-terminating simulation has unavoidable initialization bias, and then testing this presumption. Furthermore, consider the tradeoff between precision and computational budget associated with truncation points. It is common that more data will support a better analysis by obtaining a robust estimate of descriptive statistics in output analysis. However, truncation clearly implies that less data is available.

To compensate the loss of precision, faster analysis is possible with automated truncation identification, rather than human intervention or post-analysis. This kind of trade-off can be applied to the comparison between a batch means method and a replication/deletion method. However, both methodologies can be performed well after we 
find a right truncation point. We will mention the list of numerous approaches of truncating initially biased data sets in the next section, but the explanation of each method cannot be studied here unless the methods are closely related to the topic of MSER.

We might think of another aspect to investigate the characteristic of transient states prior to the steady-state in simulation output. In order to understand the path during the transient period, artificial intervention during simulation would be desirable or feasible. If so, we would like to monitor the differentiated simulation paths to an expected pre-specified steady state or a newly designated steady state from the modification of simulation input conditions. 


\section{Chapter 2: Literature Review}

The start-up problem has been the subject of research and debate for over 60 years. Early work by Morse (1955), Conway (1963), Tocher (1963), and Cohen (1982) proposed heuristics for determining the presence and persistence of an initial transient in a simulation output series. Pawlikowski (1990) reviewed eleven such rules and illustrated the strengths and weaknesses of these different approaches. He distinguished between methods based on the convergence of estimators for the sample mean and sample variance. The first set of methods included those from Emshoff and Sission (1970), Fishman (1973), Wilson and Pritsker (1978), Kelton and Law (1983), and Solomon (1983); the second set included those from Billingsley (1968), Gordon (1969), Fishman (1971), and Schruben (1982, 1983).

Pawlikowski's comprehensive review subsequently has been updated by Hoad et al. (2008) and by Pasupathy and Schmeiser (2010) to include newer approaches. The interested reader is referred to these works. In the remainder of this chapter, therefore, we focus on the literature directly related MSER.

\subsection{MSER: Approach, Inception, and Development}

At the University of Virginia (UVA), MSER was devised by Maclarnon (1990) and called the Minimal Confidence interval Rule (MCR). White and Minnox (1994), White (1995), White (1997), Rossetti et al. (1995), Spratt (1998), Cobb (2000), White et al. 
(2000), and Franklin (2009) all improved and/or further tested the approach. We begin by describing the MSER concept.

White and Minnox (1994) suggested that the optimal truncation should minimize the half-width of the marginal confidence interval about the truncated sample mean. Given the output of a simulation, a finite stochastic sequence $\left\{X_{\mathrm{i}}, i=1,2, \ldots, n\right\}$, they defined the optimal truncation point as

$$
d^{*}=\underset{n>d \geq 0}{\arg \min }\left[\frac{z_{\alpha / 2} s(d)}{\sqrt{n-d}}\right]
$$

where $z_{\alpha / 2}$ is the $z$-score of standard normal distribution associated with a $100(1-\alpha) \%$ confidence interval. The marginal standard error in the mean of the reserved sequence (i.e., the sequence remaining after truncation) is

$$
s(d)=\sqrt{\frac{\sum_{i=d+1}^{n}\left(X_{i}-\bar{X}_{n, d}\right)^{2}}{n-d-1}},
$$

and the truncated sample mean is

$$
\bar{X}_{i, d}=\frac{1}{n-d} \sum_{i=d+1}^{n} X_{i}
$$

While recognizing that, for a correlated sequence, the sample standard deviation is biased estimator of the steady-state standard deviation, they reasoned that this statistic could be interpreted instead as capturing the homogeneity of a sequence - initial sequences with larger sample standard deviations could be flagged as including transient observations. Franklin and White (2008) subsequently confirmed this intuition.

For a preconditioned confidence level, $z_{\alpha / 2}$ is a constant and Eq. 4 reduces to 


$$
d^{*}=\underset{n>d \geq 0}{\operatorname{argmin}}\left\lfloor\frac{1}{(n-d)^{2}-(n-d)}\left(\sum_{i=d+1}^{n} X^{2}-(n-d) \bar{X}_{n, d}^{2}\right)\right\rfloor .
$$

For a given output sequence from one replication of simulation, $d^{*}$ (if it exists) minimizes the constrained optimization problem in either Eq. (4) or Eq. (6). Thus, the truncation algorithm has a simple interpretation and does not require the specification of unknown parameter settings.

Spratt (1998) introduced MSER- $b$, using the means of batches of size $k$ as output variables to which MSER is applied. Batching prewhitens the output series, which is widely believed to improve the visualization of a transient (Welch, 1981). The formula was the same as Eq. (6), replacing $X_{i}$ with $Z_{j}$ (White et al., 2000),

$$
Z_{j}=(1 / b) \sum_{p=1}^{b} Y_{b(j-1)+p}
$$

where $\left\{Z_{j}, j=1, \ldots, m\right\}$ represent a series of batch means each with the size of batches $b, n$ is the number of observations of $Y$, and $m=\lfloor n / k\rfloor$ is the number of batches, where $\lfloor\cdot\rfloor$ is a maximum integer or floor function.

Independent research outside of UVA has affirmed the effectiveness of MSER-5. For example, Mahajan and Ingalls (2004) noted the efficiency and robustness of MSER-5. Oh and Park (2006) compared their exponential variation rate (EVR) rule with MSER-5 and acknowledged that the EVR only converged to the path of MSER-5. Bertoli, Casale, and Serazzi $(2007,2009)$ implemented MSER-5 into their Java Modeling toolkit.

In the U.K., Hoad et al. (2008) performed a comprehensive and detailed survey on start-up approaches, identifying over forty-six different methods as indicated in 
Table 2.1. One of the key performance indicators was whether or not an approach supported automation in order that it might be incorporated in commercial simulation software. This requirement eliminated approaches requiring a priori specification of unknown parameter settings. Among the remaining approaches, they found that MSER-5 performed exceptionally well on a wide variety of test cases. They concluded that MSER5 was consistently the best approach across the board, in terms of accuracy, robustness, simplicity, and ease of automation.

Franklin et al. (2009) demonstrated empirically that the effect of MSER is to (approximately) minimize the mean-squared error (MSE) in the estimated sample mean, which is a widely accepted criterion for a quality of a point estimate. This observation subsequently was proven analytically (under mild assumptions) by Pasupathy and Schmeiser (2010). White and Robinson (2010) reiterated the strength of MSER-5 with the basic but fundamental example of an M/M/1 queue, while White and Franklin (2010) demonstrated a parametric function which gives rise to a close-form solution accounting for geometrically decaying bias of the AR(1) process. Sanchez and White (2011) identified adjustments needed to account for differing sample sizes when applying MSER using a replication/deletion approach for interval estimation of the steady-state mean.

The theoretical work undertaken by Pasupathy and Schmeiser (2010) represents an important advance over previous empirical research. They verified analytically that the MSER statistic is asymptotically proportional to the MSE and the minima of each tend to lie close to the same truncation point. In a more recent presentation, Pasupathy and Schmeiser (2014) demonstrated analytically that MSER outperforms even two ideal 
methodologies (i.e., ideal deletion and optimal constant deletion) with uncertainty in output analysis.

Table 2.1 Methods for Determining Start-up Periods (after Hoad et al. (2008))

\begin{tabular}{|c|c|}
\hline Method Type & Method \\
\hline Graphical & $\begin{array}{l}\text { Simple Time Series Inspection } \\
\text { Ensemble (Batch) Average Plots } \\
\text { Cumulative-Mean Rule } \\
\text { Deleting-The-Cumulative-Mean Rule } \\
\text { CUSUM Plots } \\
\text { Welch's Method } \\
\text { Variance Plots (or Gordon Rule) } \\
\text { Exponentially Weighted Moving Average Control Charts } \\
\text { Statistical Process Control Method (SPC) }\end{array}$ \\
\hline Heuristic & $\begin{array}{l}\text { Ensemble (Batch) Average Plots with Schribner's Rule } \\
\text { Conway Rue or Forward Data-Interval Rule } \\
\text { Modified Conway Rule or Backward Data-Interval Rule } \\
\text { Crossing-Of-The-Mean Rule } \\
\text { Autocorrelation Estimator Rule } \\
\text { Marginal Confidence Rule or Marginal Standard Error Rule (MSER) } \\
\text { Marginal Standard Error Rule m, (e.g. m = 5, MSER-5) } \\
\text { Telephone Network Rule } \\
\text { Relaxation Heuristics } \\
\text { Beck's Approach for Cycle output } \\
\text { Tocher's Cycle Rule } \\
\text { Kimbler's Double exponential smoothing method } \\
\text { Euclidean Distance (ED) Method } \\
\text { Neural Networks (NN) Method }\end{array}$ \\
\hline Statistical & $\begin{array}{l}\text { Goodness-Of-Fit Test } \\
\text { Algorithm for a Static Dataset (ASD) } \\
\text { Algorithm for a Dynamic Dataset (ADD) } \\
\text { Kelton and Law Regression Method } \\
\text { Glynn \& Iglehart Bias Deletion Rule } \\
\text { Wavelet-based spectral method (WASSP) } \\
\text { Queueing approximations method (MSEASVT) } \\
\text { Chase Theory Method (methods M1 and M2) } \\
\text { Kalman Filter method } \\
\text { Randomization Tests For Initialization Bias }\end{array}$ \\
\hline Initialization bias test & $\begin{array}{l}\text { Schruben's Maximum Test (STS) } \\
\text { Schruben's Modified Test } \\
\text { Optimal Test (Brownian bridge process) } \\
\text { Rank Test } \\
\text { Batch Means Based Tests - Max Test } \\
\text { Batch Mean Based Test - Batch Means Test } \\
\text { Batch Means Based Test - Area Test } \\
\text { Ockerman \& Goldsman Students t-test Method } \\
\text { Ockerman \& Goldsman (t-test) Compound Tests }\end{array}$ \\
\hline Hybrid & $\begin{array}{l}\text { Pawlikowski’s Sequential Method } \\
\text { Scale Invariant Truncation Method }\end{array}$ \\
\hline
\end{tabular}


In its original form (Eqns. 4 and 6), the MSER criterion suggests the choice of a truncation point is the global minimum of the MSER statistic on $n>d \geq 0$. (This choice we shall denote as MSER-GM.) It was recommended in practice, however, that the choice of a truncation point be constrained to the first half of the output sequence, $d<n / 2$. (This choice we shall denote as MSER-Half.) The reasoning was that, if a minimum is not found on this interval, the run length is insufficiently long to provide a tight interval estimate, possibly because the simulation is unstable and no suitable truncation point exists. Pasupathy and Schmeiser (2014) proposed and tested two additional alternatives: the leftmost local minimum of the MSER statistic (MSER-LLM) and the left-most local minimum of the local minima of the MSER statistic (MSER-LLM2).

They suggested that MSER-LLM was the best choice. However, their intention to use the most left local minimum also confirmed the notion of checking the minimum value prior to the first half of output series in MSER-Half when the sample size is enough to obtain a steady-state mean estimate after preprocessing data from simulation with a reasonable batch size. That is, the relationship among the truncation points is $b_{L L M} \leq b_{L L M 2} \leq b_{G M}$ (Pasupathy and Schmeiser, 2010), where $b_{\mathrm{GM}}$ is the truncation point that yields a globally minimum MSER statistic. After properly batching output and generating a sufficient sample size, $b_{L L M}$ would be equivalent to $b_{G M}$ or $b_{H a l f}$.

\subsection{Open Issues}

The motivation for this research stems from a range of issues that have been raised regarding various aspects of MSER. Insight on how to resolve these issues is the subject of the subsequent chapters and a contribution of this research. 


\subsubsection{Implementation and automation}

As noted in the Chapter 1, Hoad and Robinson (2008) explored the practical implementation in commercial simulation software and Hoad et al. (2011) provided a framework to automate an output analyzer incorporating the logic of MSER-5. They identified four obstacles to implementation. However, as we demonstrate in this research, these appear to be specific to limitations of the simulation software they employed (SIMUL8, see Figure 2.1) and not innate difficulties caused by MSER-5.

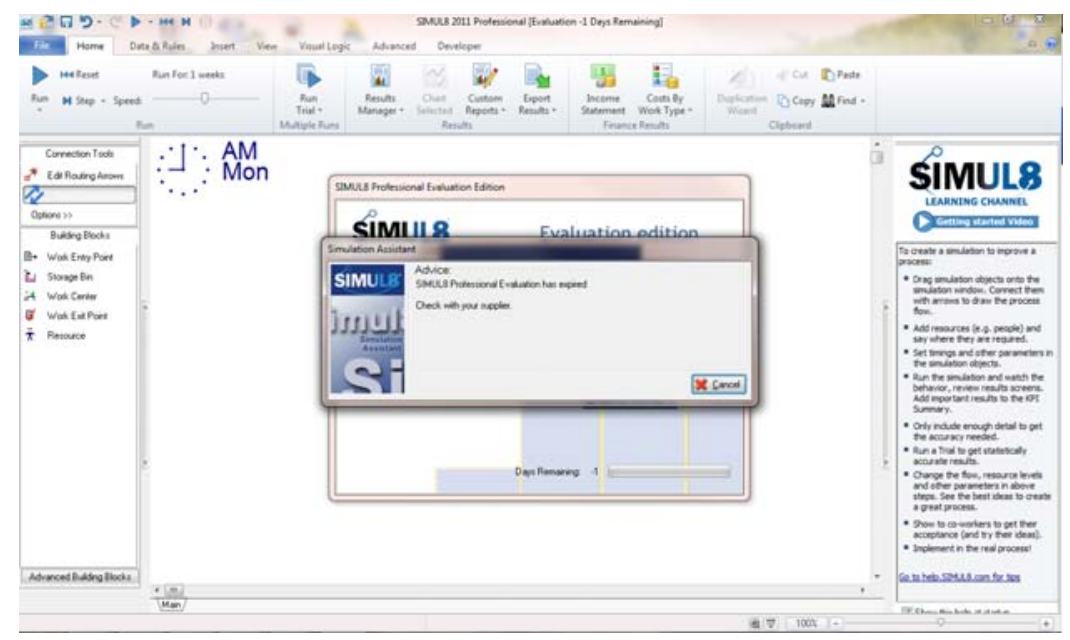

Figure 2.1 Testing Module for identifying optimal truncation points in SIMUL8.

The difficulties identified were:

(1) selection of the simulation run length selection,

(2) sequential data collection from multiple replications,

(3) output types associated with cumulative values and extrema,

(4) data associated with entities.

The first difficulty is fundamental in simulation (and sampling more generally) and not unique to the question of automating a warm-up procedure for steady-state simulation. We 
illustrate this difficulty specifically as it relates to MSER in Chapter 7 and acknowledge the run-length selection remains an open and perhaps unavoidable problem. However, to present this difficulty as an absolute barrier to automation is disingenuous, insomuch as it denies the practicality and usefulness of any form of automated output analysis, which clearly is not the case. With this caveat, in Chapters 5 and 6 we demonstrate both theoretically and by application that the remaining barriers are readily overcome.

The second (and fourth) difficulties easily can be handled by storing vectors of raw output data with time stamps. If the software application enables a modeler to save variables of his/her interest, these issues should not be regarded as any obstacle. Visual Logic Editor in SIMUL8 might also provide the functionality to export raw data after each replication, or any state change, and exporting data or saving online output in memory would alleviate the second issue. Moreover, MSER is best applied to individual output sequences and there is no need to save within-run observations across replications (see Section 2.4).

The third difficulty arises in the desire to avoid malpractice by novice simulationistsa difficulty that will never arise in a well-conceived output analysis. A cumulative statistic $\left\{Y_{i}: i=1,2, \ldots, n\right\}$ is derived from an underlying output sequence $\left\{X_{i}, i=1,2, \ldots, n\right\}$, and it is this latter sequence to which MSER is applied to determine a truncation point, not to the former. The same is true of extrema.

\subsubsection{Batch Size}

Spratt (1998), White, Cobb, and Spratt (2000), and most recent papers advocate MSER$b$, which prewhitens the output sequence by creating averages of non-overlapping batches 
of size $b$. Their empirical results suggest that MSER-5 is modestly better than MSER without prewhitening. However, Pasupathy and Schmeiser (2010) note

that any one-size-fits-all preprocessing, however, leads to a contradiction: if the preprocessed data are better in some sense, then why not preprocess the preprocessed data? If preprocessing is to make sense, then its form needs to be based on an analysis of the given data $\left\{X_{1}, X_{2}, \ldots, X_{n}\right\}$. Another point is that the use of non-overlapping batches is suboptimal to using overlapping batches, which leave no orphaned observations at the end of the data series, which cause no graininess in the analysis, and which still requires only $O(n)$ computation.

Their logic appears unassailable and the question of an optimal batch size $b$, its relationship to given data, and the overlapping batch means (OBM) approach will be further explored in Chapter 7. Originally proposed by Meketon and Schmeiser (1984), OBM replaces Equation (7) with

$$
O B M_{k}=(1 / b) \sum_{p=1}^{b} Y_{b+p-1}
$$

where $\{O B M k, k=1, \ldots,(n-b+1)\}$ represents a series of batch means for overlapping batches of size $b$, where $n$ is the number of observations of $Y_{i}$. In general, the $O B M_{k}$ are highly correlated. The correlation is accounted by computing the variance estimator as

$$
S_{O B M}^{2}=\frac{b \sum_{k}^{n-b+1}\left[\bar{X}_{k, d}-\overline{\bar{X}}\right]^{2}}{(n-b+1)(n-b)}
$$

and the $(100-\alpha) \%$ confidence interval as

$$
\overline{\bar{X}}_{n, k} \pm t_{f, 1-\alpha / 2} \sqrt{S_{O B M}^{2}}
$$


where $t_{f}$ is distributed Student-t distribution with $f$ degrees of freedom (see Law, 2015).

In this regard, the literature on batch means also may provide some insights. The batchmeans method is aimed at mitigating serial correlation between output data in simulation by batching the output data sequentially and using the means and of these batches in the construction of interval estimates of the true steady-state mean. Schmeiser (1982) investigated batch size effects. In other words, given a sequence in steady-state (after first truncating any transient), how many batches are required and how wide must the batches be in order to obtain an unbiased estimate to meet any prescribed condition for a simulation model? Goldsman and Meketon (1986), Schmeiser and Song (1987), Song and Schmeiser (1993, 1995), Song (1996), and Nelson (2011) have discussed the batch size effect and optimality condition in batch mean methods.

\subsection{Current Related Work}

\subsubsection{N-SKART}

Comparatively recently, James Wilson and his students at North Carolina State University have developed new truncation algorithms that appear to show modestly better performance than MSER on select problem instances. These methods derive from WASP, SBatch, and SKART, and include N-SKART, and MSER5Y (see Figure 2.2). In particular, Mokashi et al. (2010) compared N-SKART to MSER in terms of performance criteria such as the success rates of finding truncation points, the minimal MSE values, and confidence interval coverage.

We do not pursue direct empirical comparisons with NSKART or MSER-5Y in this research, at least in part because of the extreme complexity of these methods. We simply 
note in passing that, where improvements over MSER were in fact demonstrated, the test cases employed appear inherently unrealistic. Moreover, improvements were at best marginal and required vastly greater computational effort. Further, their algorithm has an innate weakness in that it does not detect any bias in the moving average of output series.

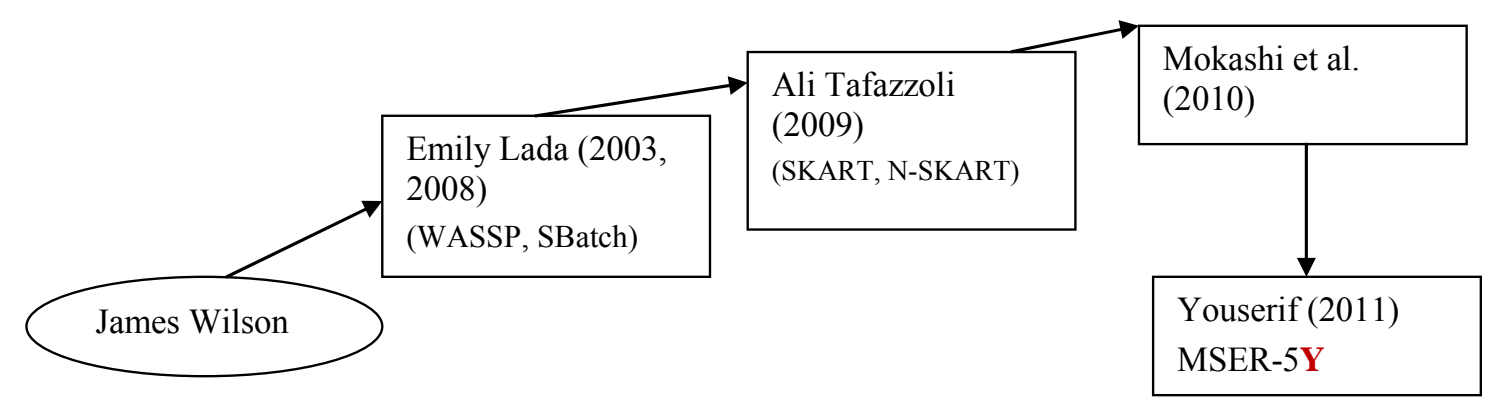

Figure 2.2 Output Analysis Tree for Wilson and Students

\subsubsection{Potentially Insufficient Truncation}

Recent work by Law (2015) and Wang and Glynn (2014) suggests that MSER may not truncate an appropriate number of observations. Both studies apply theoretical constructs that focus on the average truncation points that should be obtained given an infinite number of replications. As demonstrated by White and Hwang (2015), this suggestion appears to contradict both the theoretical results obtained by Pasupathy and Schmeiser (2010) and the very substantial body of empirical evidence accumulated over the past twenty-five years. Nevertheless the suggestion merits investigation and further discussion is undertaken Chapter 7. 


\subsection{Optimal Analysis for the Mean of a Simulation Output}

White (2012) demonstrates how output analysis can be formulated as a constrained optimization problem. We include his formalism here because it not only provides a notation for the overall problem, but also a means to determine memory requirements for implementing MSER. The objective is to achieve an estimate of the mean for a simulation output with a given precision using the least number of observations. We consider three cases - terminating simulations, nonterminating simulations using the replication/deletion approach, and nonterminating simulations using the batch means approach.

\subsubsection{The terminating simulation problem}

We are given user-defined values for:

- $H W$ and $\alpha$, where $H W$ is the maximum desired half-width of the $100(1-\alpha) \%$ confidence interval on the sample mean of the simulation output;

- $r$, the initial number of replications; and

- $R$, the maximum number of replications.

The problem is then:

Find

$$
\min _{r \in\left\{r_{0}, r_{1}, \cdots\right\}} r
$$

where $r$ is the number of replications required, such that

$$
r \leq R
$$




$$
H W(r)=t_{r-1,1-\alpha / 2} \sqrt{\frac{S^{2}(r)}{r}} \leq H W .
$$

Here $H W(r)$ is the estimated half-width derived from $r$ replications,

$$
S^{2}(r)=\frac{1}{r-1} \sum_{j=1}^{r}\left(\bar{X}_{j}\left(n_{j}\right)-\overline{\bar{X}}(r)\right)
$$

is the variance of mean of the sample means,

$$
\bar{X}_{j}\left(n_{j}\right)=\frac{1}{n_{j}} \sum_{i=1}^{n_{j}} X_{i j} ; j=0,1,2, \ldots, r
$$

are the sample means, and

$$
\overline{\bar{X}}(r)=\frac{1}{r} \sum_{j=1}^{r} \bar{X}_{j}\left(n_{j}\right)
$$

is the grand mean.

Note that this optimization problem may be infeasible for a given choice of $H W, \alpha$, and $R$.

If this is the case, the user has the option of relaxing any or all of these three parameters, i.e., increasing the number of replications and/or settling for a less precise estimate. Note also that an estimate for the number of additional runs required at any stage $i+1$ is

$$
r_{i+1}=\left(\frac{H W(r)}{H W}\right)^{2} r_{i}-r_{i}
$$

If $r_{i+1}>R$, then let $r_{i+1}=R$. This is likely a more efficient approach than incrementing $r$ by a fixed amount. Note finally that the data required to solve this optimization problem is essentially the $r$-dimensional array of sample means $\left\{\bar{X}_{j}\left(n_{j}\right)\right\}$. 


\subsubsection{The steady-state simulation problem: replication/deletion approach}

This is the same as the terminating simulation problem, except that we must now determine suitable initial and terminating conditions for each replication by solving a second set of optimization problems. We are given user-defined values for $H W, \alpha, R$, and

- $\quad n$, the initial run length for each replication

- I, the MSER re-computation interval

- $\quad N$, the maximum number of such intervals (corresponding to a tentative stopping condition for a maximum run length of $n_{0}+N I$ )

For each replication $j=1, \ldots, r$, first solve the following problem:

Find

$$
\min _{n_{j} \in\left\{n_{0}, n_{0}+I, \ldots, n_{0}+N I\right\}} n_{j}
$$

where $n_{j}$ is the run length required on the $j^{\text {th }}$ replication, such that

$$
d_{j} *\left(n_{j}\right)=\underset{\left\lfloor n_{j} / k\right\rfloor d \geq 0}{\operatorname{argmin}}\left[\frac{S_{j}^{2}\left(n_{j}, d_{j}\right)}{n_{j}-d_{j}}\right] \leq\left\lfloor n_{j} / k\right\rfloor
$$

Here $d_{j} *\left(n_{j}\right)$ is the MSER-optimal truncation point for replication $\mathrm{j}$ with run length $n_{j}$, where $\lfloor$.$\rfloor is the floor function,$

$$
S_{j}^{2}\left(n_{j}, d_{j}\right)=\frac{1}{n_{j}-d_{j}} \sum_{i=d_{j}+1}^{n_{0}+n_{j}}\left(X_{i j}-\bar{X}_{j}\left(n_{j}, d_{j}\right)\right)^{2}
$$

is the truncated sample variance, and

$$
\bar{X}_{j}\left(n_{j}, d_{j}\right)=\frac{1}{n_{j}-d_{j}} \sum_{i=n_{j}-d_{j}+1}^{n_{j}} X_{i j}
$$


is the truncated sample mean.

Note that constraint Eq. (17) implies the existence of an optimal MSER truncation point on the output series $X_{1}, X_{2}, \ldots, X_{\left\lfloor n+n_{0} / k\right\rfloor}$. The best choice for $k$ is unclear, but we have achieved good results by requiring the truncation point to be within the first half of the output time series, i.e., $k=2$.

Furthermore, note that failure to satisfy constraint Eq. (17) implies that the run length is insufficient, or that the output is unstable. Without some insight into the nature of the output, there is no way of knowing which is the case. If constraint Eq. (17) is not satisfied, the user may choose to increase the value of $N$, or conclude the output is unstable and stop. There is no need to evaluate constraints Eq. (11) and Eq. (12) if constraint Eq. (17) is not satisfied.

Note also that when computing the confidence interval using this approach, one needs to account for that fact that MSER will yield replications of unequal sample size. See Sanchez, P. J., and White, K. P., Jr. (2011) for one approach this issue. If the desired $H W$ constraint is not achieved, additional runs may be attempted. Note finally that the data required is the output time series $X_{0}, X_{1}, \ldots, X_{n+n 0}$. For time-persistent statistics, we can construct this from the array of pairs $\left\{x_{i}, t_{i}\right\}$.

\subsubsection{The steady-state simulation problem: batch means approach}

This is the same as the replication/deletion problem, except that we must now implement batching. By construction, $r_{0}=R=1$, constraint Eq. (11) is automatically 
satisfied, and the subscript $j$ in constraint Eq. (17) is superfluous. Constraint Eq. (12) is replaced by

$$
H W\left(n, d^{*}\right)=t_{n-d^{*}-1,1-\alpha / 2} \sqrt{\frac{S_{B}^{2}\left(\left\lfloor n / B, d^{*}\right\rfloor\right)}{\lfloor n / B\rfloor}} \leq H W
$$

where

$$
S_{B}^{2}\left(\left\lfloor\left(n-d^{*}\right) / B\right\rfloor\right)=\frac{1}{\left.\left(n-d^{*}\right) / B\right\rfloor} \sum_{j=1}^{\left\lfloor\left(n-d^{*}\right) / B\right\rfloor}\left(Z_{j}-\bar{Z}\left(\left\lfloor\left(n-d^{*}\right) / B\right\rfloor\right)\right.
$$

is the standard deviation of the batch means using $B$ batches, given by

$$
Z_{j}=\frac{1}{B} \sum_{i=j+1}^{j+B} X_{i}
$$

Note that Arena has a well-documented algorithm for adjusting the batch size $B$ as a function of the run length that maintains $40>B \geq 20$. See Kelton et al. (2010, pp.326-327), for the algorithm and rationale. 


\section{Chapter 3: MSER Implementation Issues}

In this chapter we consider various options for implementing MSER truncation logic within alternative software environments. Broadly, we distinguish between two such options. One is to compute and update a truncation point while simulating a model (i.e., online analysis). The other is to analyze the output data obtained after simulating the model (i.e., post-analysis).

With respect to online analysis, we distinguish between external and internal implementation approaches. We provide an example of the external approach by outlining development of a dynamic linked library (DLL) that performs MSER calculations. This DLL can be called from within models built in Windows-based commercial languages such as ProModel and MedModel. For internal implementations, we identify the software development environments associated with a wide range of the most popular commercial DES languages, including Arena, AutoMod, SIMUL8, ProModel (MedModel), FlexSim, Simio, ExtendSim, and SimCAD. With respect to post-analysis, we review five different languages in which we have written standalone programs in which we implement MSER: $\mathrm{R}, \mathrm{C}++$, Matlab, SAS, and VBA.

This chapter provides background leading to several selective implementations. These implementations were developed as part of this research and are distributed online via the MSER Laboratory. The details of these implementations and the Lab are provided in Chapters 4-6 and the Appendices. 


\subsection{Online Analysis}

Using online analysis, MSER statistics are continuously updated during each simulation run. These statistics are used to determine a truncation point following the logic provided in Section 2.4. If such a point exists, the simulation terminates and output statistics are reported, allowing comparison of the estimated steady-state mean and corresponding confidence interval with and without truncation. If such a point does not exist, this fact is reported instead. Online analysis can be implemented in two different ways.

\subsubsection{External Approach}

DES software increasingly is extendable using external modules, such as dynamic linked libraries (DLL) and Component Object Models (COM). In particular, on a Windows operating system DLL's have the flexibility to provide new functions and variables in order to obtain intended simulation objectives (e.g., additional complex computation). A single DLL can contain multiple new functions and multiple programs can share the same DLL. We note, however, that DLL's must be used with caution. Any modification to an existing DLL must not adversely affect a previously linked application. Therefore, we need to emphasize the careful documentation of the DLL. This is essential to maintain programming intent and any logic to consist of inside functions. Without this practice, it is difficult to use DLL across different applications, as a compiled file does not demonstrate how it works or what it accomplishes. 


\subsubsection{How to build DLL linked with ProModel (MedModel)}

ProModel (http://www.promodel.com/) has a function, $\operatorname{XSUB(~),~which~is~used~call~}$ external DLL files. This command provides modelers with more versatile power to control simulation models, such as performing a complex calculation separately without burdening simulating a model. Furthermore, one standard and representative DLL will be portable to additional simulation models. To use this feature, the modeler must know how to code DLL files in a high-level programming language. The exemplar DLL file is written in $\mathrm{C} / \mathrm{C}++$, but it can be written in other programming languages as well. Furthermore, its portability will be very useful to be foster reusability continuously (ProModel, 2011).

Those who are new to Promodel and the usage of XSUB( ) can refer to the manual in Promodel. However, this command is considered as an advanced option so that we would like to explain general ideas here. One of the strengths of an XSUB( ) external subroutine call is in enhancing the capability of ProModel through the users' programming skills. That is, as long as the programing language (i.e., $\mathrm{C} / \mathrm{C}++, \mathrm{VB}$, and Pascal) is supportive, the logic called with $\mathrm{XSUB}()$ enables a modeler to test every intention, such as complex file IO and statistical analysis.

Its principal limitation is that, while $\mathrm{XSUB}()$ is being executed, the simulation is temporarily halted so it is important that the computation inside the external subroutine doesn't take too long. A Windows 32-bit compiler must compile the logic inside the DLL and a user must understand Windows platform knowledge.

The function representing the logic only takes one parameter, a void pointer (a generic pointer). However, it may access multiple parameters through structure. That is why the function should include a structure. This is a strict condition to implement a user's 
intention. In addition to MSER DLL in a following chapter, a basic example of DLL usage is explained (ProModel, 2011).

Syntax:

$\operatorname{XSUB}(<\mathrm{file} I D>$, <ordinal function number $>$ or $<$ function name $>\{$, <parameter1>, <parameter2>...\})

Example

XSUB(MSER，1，5) or XSUB(MSER_raw， “Log_B_of_output”，10，5)

The components of the four elements in $\mathrm{XSUB}()$ are briefly explained in syntax. The argument $<$ file ID $>$ is an identifier to build a simulation model and link its output with a DLL file. Figure 3.1 shows how to designate a file ID and set up its type as DLL. When DLL is ready, we need to specify it into "File Name".

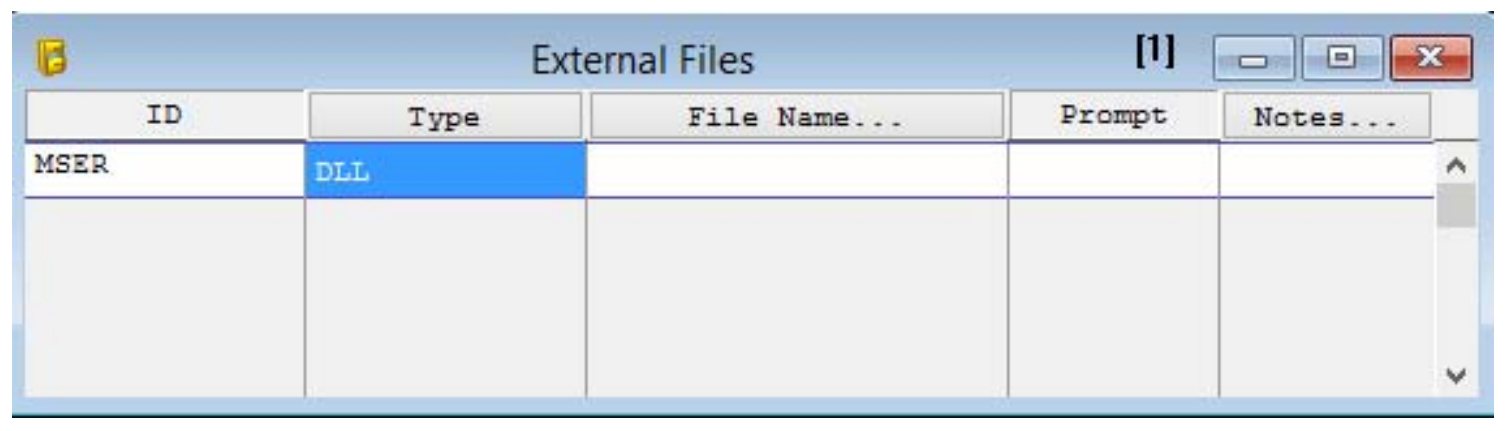

Figure 3.1 Example of DLL usage in ProModel

The argument $<$ ordinal function number $>$ or $<$ function name $>$ is used to set up which function inside a DLL file becomes interactive with ProModel. As we build a DLL file, multiple functions can be constructed to perform output analysis. For example, we want to find a truncation point, MSER statistics, or a truncated mean value. If we build different functions to compute them, we need to remember their order and then use the ordinal 
number for relevant components of $\mathrm{XSUB}()$. As an alternative, we can use the defined names inside DLL for XSUB() components. The argument $<$ parameters $>$ is any set of input values generated from ProModel to a DLL file. It will be passed to the function of DLL.

\subsubsection{Internal Approach}

The internal approach can be achieved in several different ways. First, the software developer can incorporate MSER logic for release in a version upgrade. This clearly is the best approach, but is limited by the ability and willingness of the software house to provide internal developers' time to create and test the upgrade. Second, a static library can be written in a software language specific to the commercial simulation suite. This is the second best approach, but at present is officially supported by only one software company, ExtendSim. Third, a submodel or subprocess can be developed to compute the MSER truncation point and associate statistics, such as in Arena. Such a submodel is reusable given in-detail explanations of submodel inputs, outputs, and operation. We pursue the second two approaches in Chapters 5.

\subsubsection{Software development environments}

The main purpose of this session is review how commercial DES are created and which languages would be used in an internal approach. We include the most popular software adopted in academia as well as industry. Most software environments are built on objectoriented programming languages such as $\mathrm{C}++, \mathrm{C} \#$, and Java. However, simulation-specific languages also are employed (Arena, for example, is built in the simulation language SIMAN, which itself was originally programmed in Fortran and then later reprogrammed in C). We determined which development language has been used in each simulation suite 
based on information in the suites' users manuals. Where these manuals are inadequate to make this determination, we also consulted each company's job postings. As most of software houses need to hire new programmers, we can infer which language is used for specific simulation software. We list the languages for each simulation environment as follows:

- Arena-SIMAN

- AutoMod - proprietary language

- SIMUL8 - proprietary language

- ProModel (MedModel) - MS Studio GUI and its proprietary language

- FlexSim $-\mathrm{C}++$ based proprietary language

- Simio - C\#

- ExtendSim - C-based proprietary language, ModL

- $\operatorname{SimCAD}-\mathrm{C} \#$

All of these DES software applications run only on MS Windows OS (and the same is true of all but a few agent-based simulation applications and open-source simulation languages). That is why more and more simulation GUIs have adopted the Window's ribbon-style interfaces. Thus, we expect that a single versatile DLL in a single objectoriented programming language to be usable across the majority of these environments.

\subsubsection{Current Features for Dealing with the Start-up Problem}

After understanding basic and fundamental structure of each software suite, we observe different approaches to deal with a warm-up period across multiple simulation software 
environments. We test Arena, AutoMod, SIMUL8, ProModel, FlexSim, Simio, and SimCAD. Almost all of these have features for specifying a warm-up period during run setup. The specifics for each language are summarized, as well as the corresponding interface.

\subsection{Arena}

Arena (https://www.arenasimulation.com/) was originally developed by Dennis Pegden at Systems Modeling Corporation and later acquired by Rockwell Software (Banks 1998; Kelton et al., 2010). It is an extensible simulation and animation software package that provides a complete simulation environment supporting all steps in a simulation study. Arena combines the modeling power and flexibility of the SIMAN simulation language with a GUI interface for drag-and-drop model building as well as simulation run animation.

In Arena, the warm-up period is defaulted to zero unless otherwise specified on the "Replications Parameters" tab in "Run Setup" menu shown in Figure 3.2. Arena has a good feature to take advantage of both replication/deletion and batch means approaches to steady-state simulation by using "Initialize Between Replication", a feature which is not seen in other software by default. 


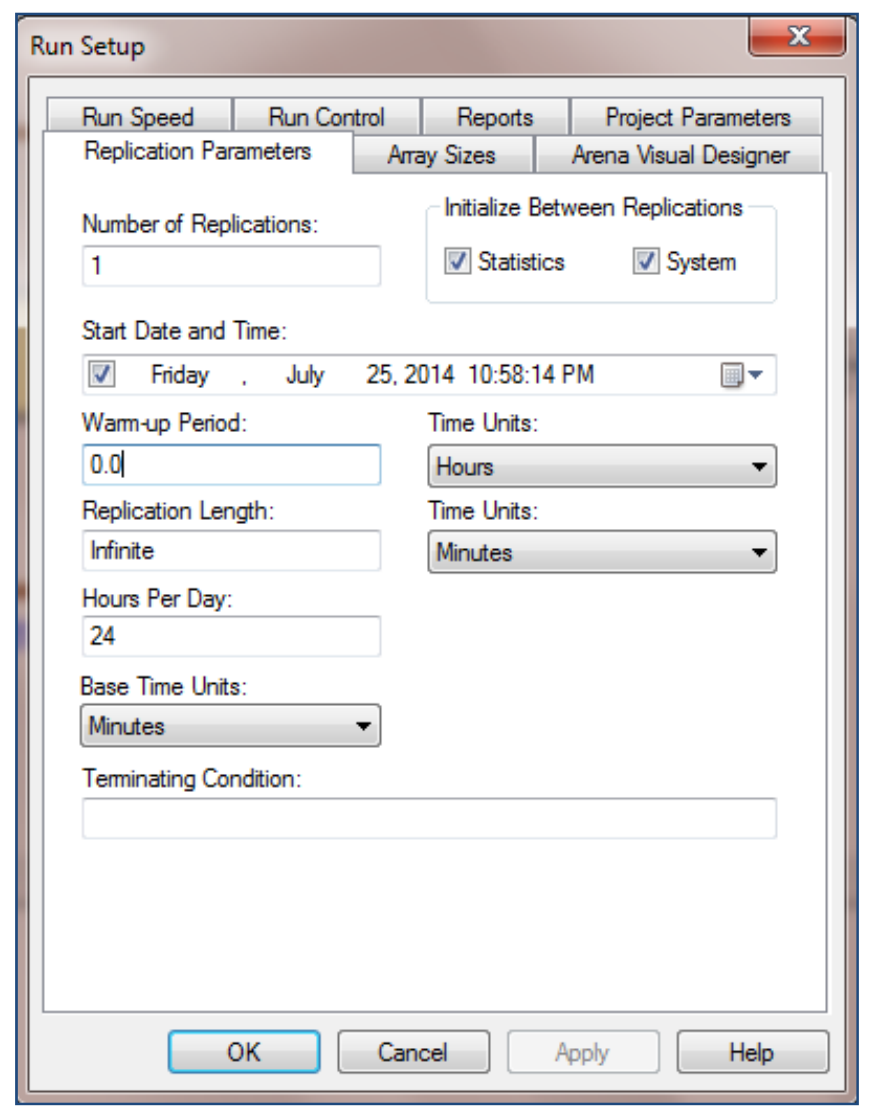

Figure 3.2 Specifying Warm-up Period in Arena's Run Setup Dialogue

\subsection{AutoMod}

AutoMod is a graphical simulation software environment providing true-to-scale 3D simulation developed by Applied Materials (http://www.appliedmaterials.com/global-

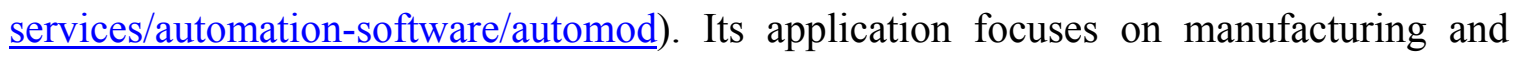
distribution operations (i.e., semiconductor industry). AutoMod puts much importance on output analysis and clearly promotes using replication/deletion method to compute its confidence interval. Comprehensive explanations of determining warm-up period are followed inside its manual - an entire chapter is devoted to addressing the warm-up problem — which is a rare case among current simulation software manuals. The AutoMod 
suite includes a companion program, AutoStat, designed specifically to implement Welch's procedure, in particular.

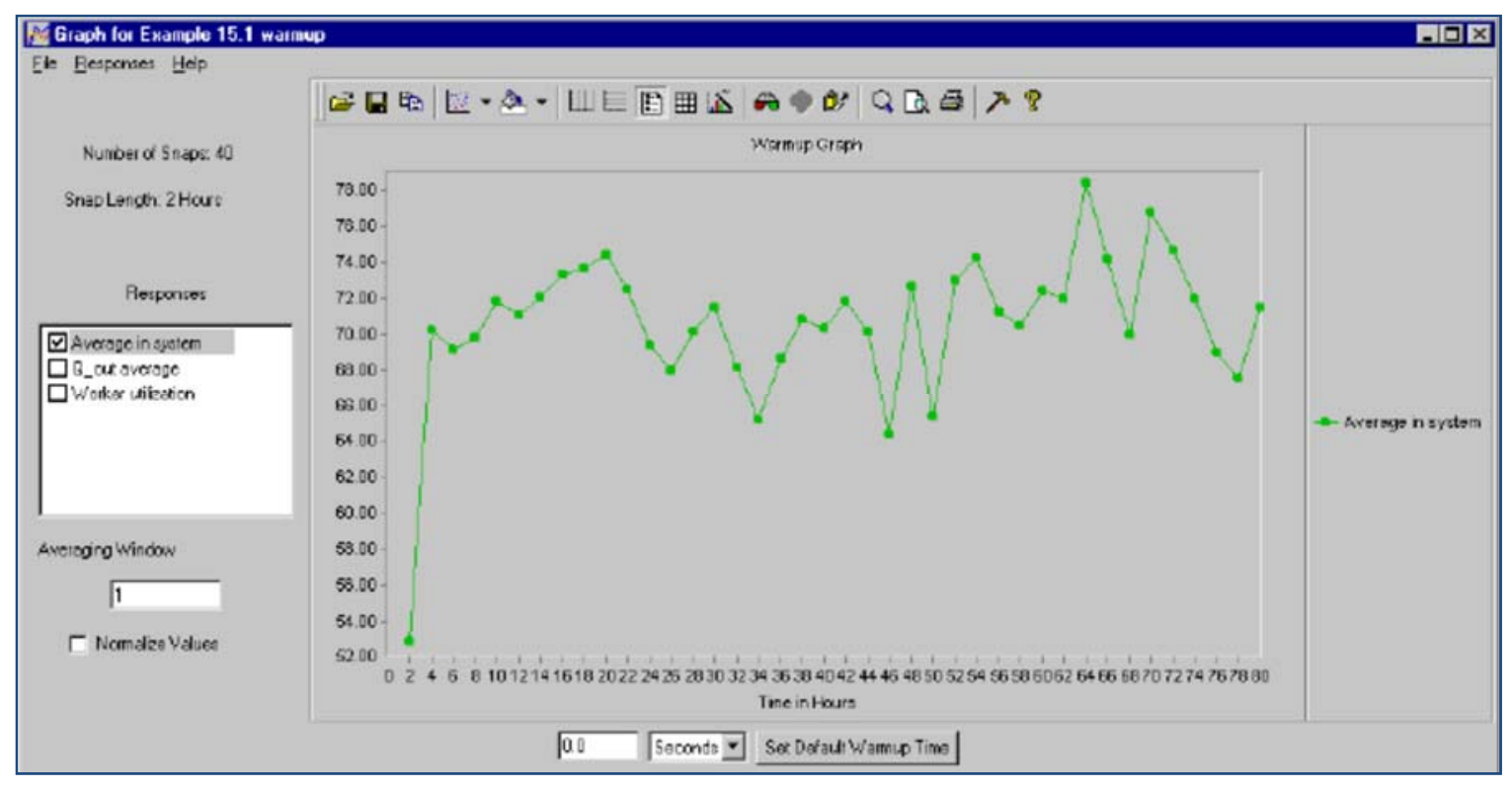

Figure 3.3 Warm-Up Period Determination in AutoMod

\subsection{SIMUL8}

SIMUL8 (http://www.simul8.com/) is a process-based DES that helps an analyst to build high-level simulation model, widely adopted as a teaching language by educational institutions in the UK. Like Arena, it has an option to specify warm-up period, as shown in Figure 3.4. As shown in Figure 3.5, SIMUL8 also includes a routine to estimate the number of simulation replications required to achieve a specified minimum precision in specified output statistics (KPI's). It is noteworthy that Hoad and Robinson (2011) relate lessons learned in their effort to implement MSER in SIMUL8. 


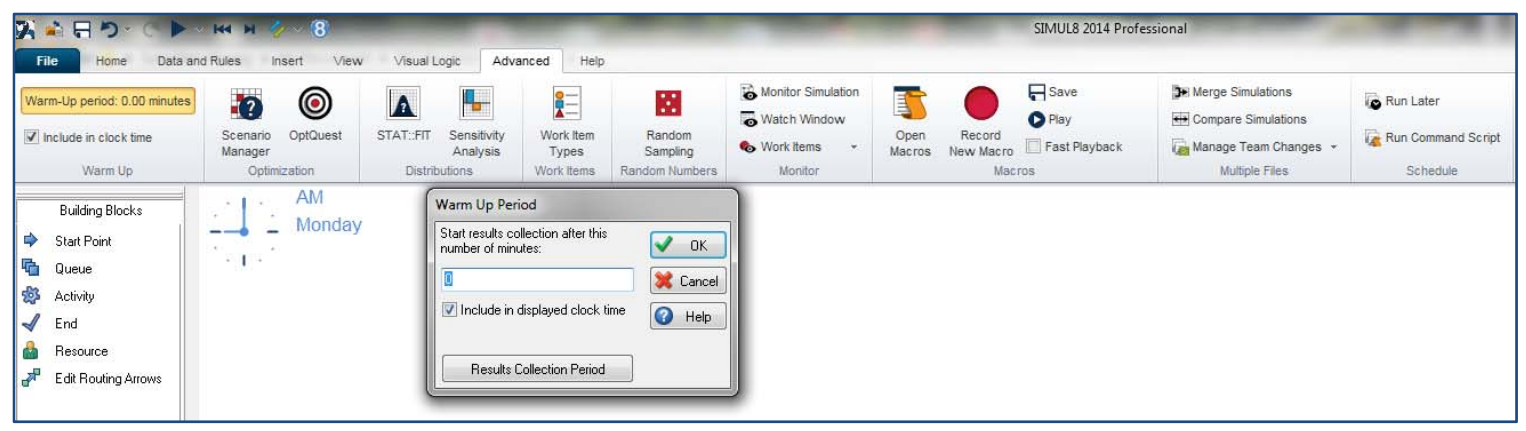

Figure 3.4 Warm-Up Period in SIMUL8

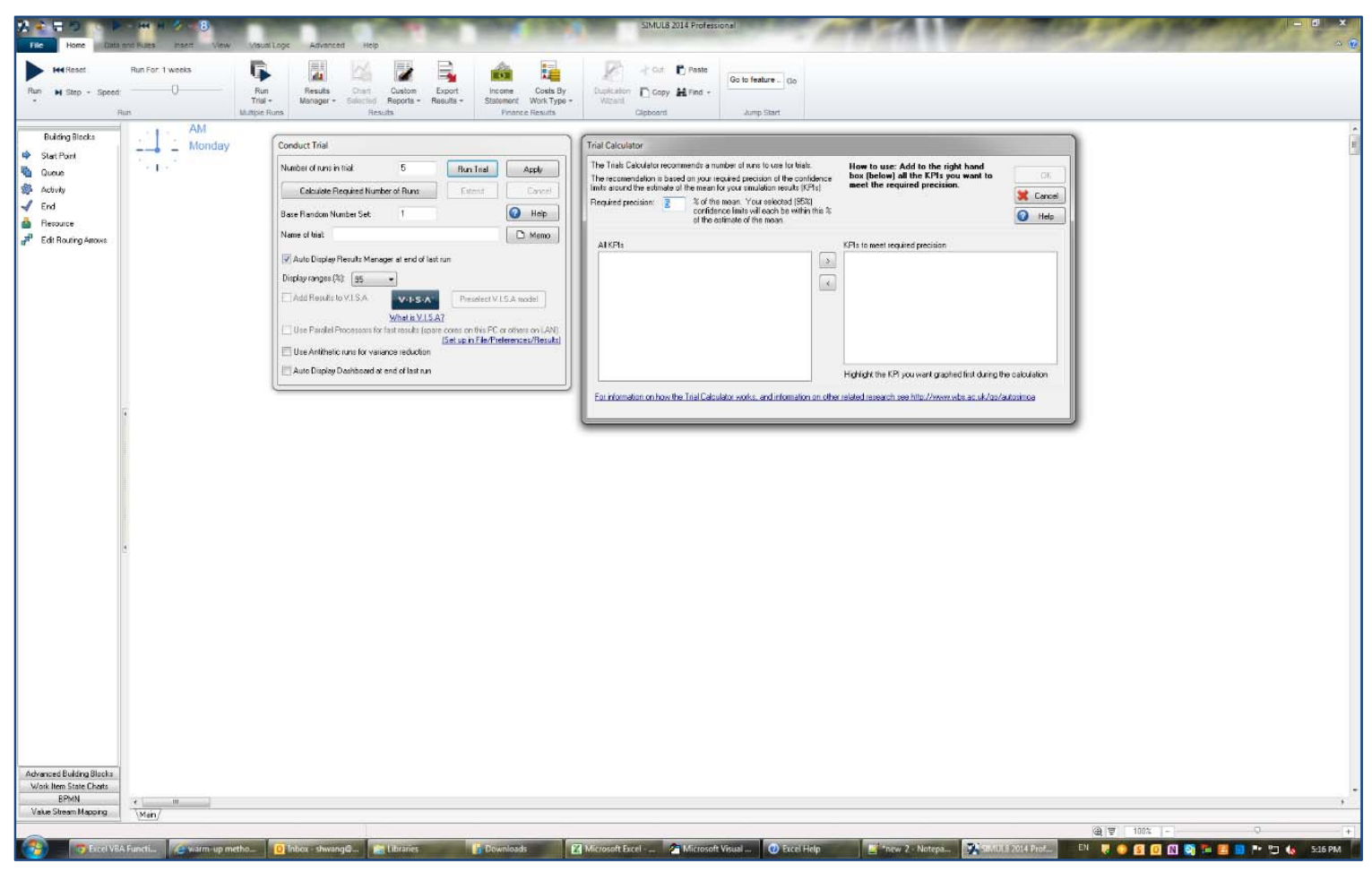

Figure 3.5 Output Analysis Support in SIMUL8

\subsection{ProModel and MedModel}

ProModel is an older DES suite that aims to provide insights on planning, designing, and improving existing or new manufacturing, supply chain, and other discrete-event 
systems. It also has a healthcare specialized version, MedModel. Its peculiar feature is the incorporation of a location-based modeling approach that allows a user to calculate spatial components simultaneously (e.g., travel distances of entities and resources). As
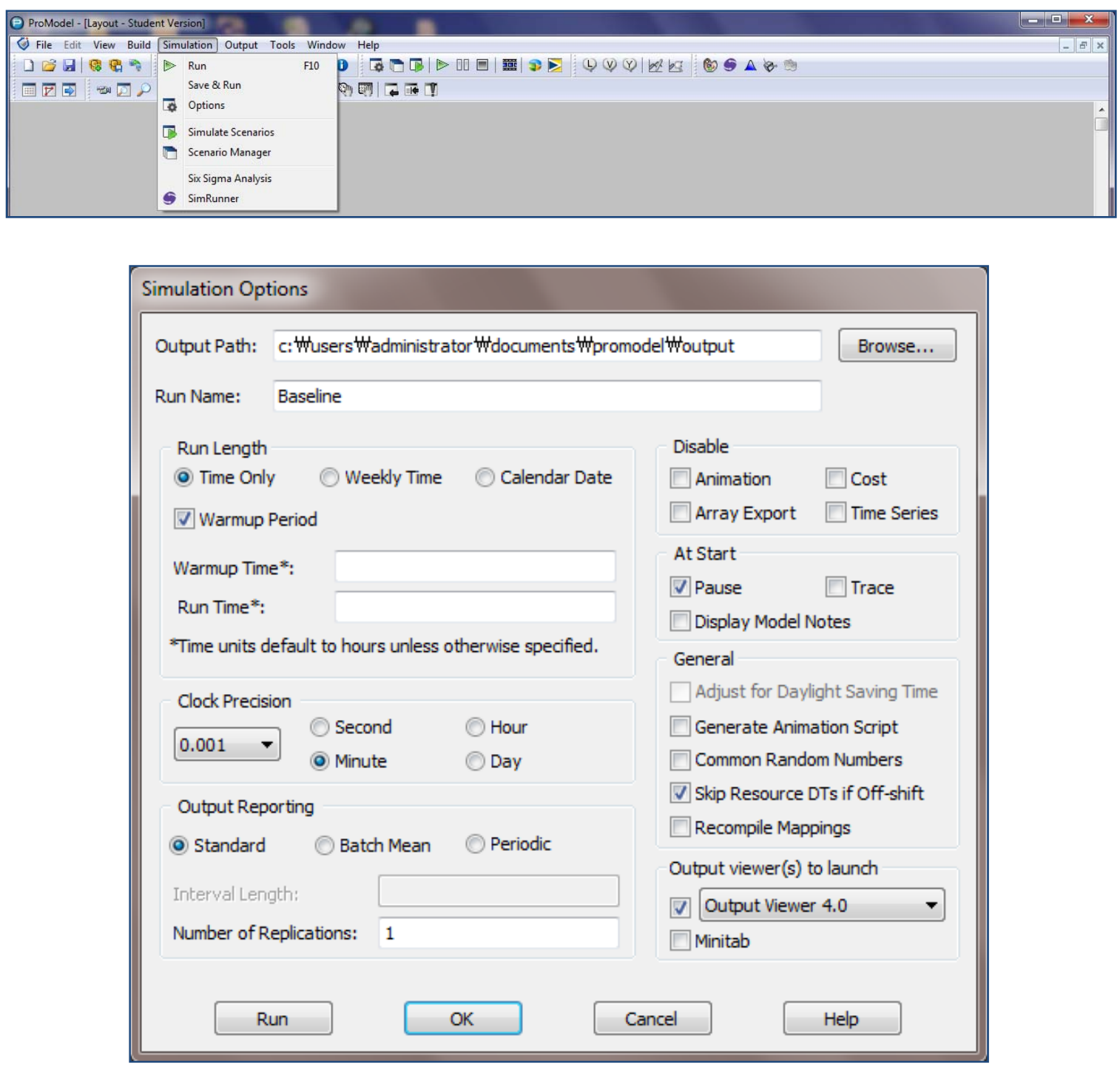

Figure 3.6 Specifying a Warm-Up Period in ProModel

shown in Figure 3.6, like most of the simulation software described, ProModel has an option to set up a warm-up time at a given simulation clock time under the simulation option window. Additionally, it allows specification of a warm-up period based on the 
number of processed entities (e.g., WARMUP). Thus, it provides dual modes to control a warm-up period.

\subsection{FlexSim}

FlexSim was founded in 1993 by Bill Nordgren (Co-Founder Promodel Corporation, 1988) along with Roger Hullinger and Cliff King. Its strength lies in 3D-modeling capability. Most of the features inside FlexSim are similar to ProModel. It supports its own language, Flexscript, as well as $\mathrm{C}++$ when a modeler builds a simulation model. Figure 3.7 shows the dialog for setting a warm-up period in FlexSim

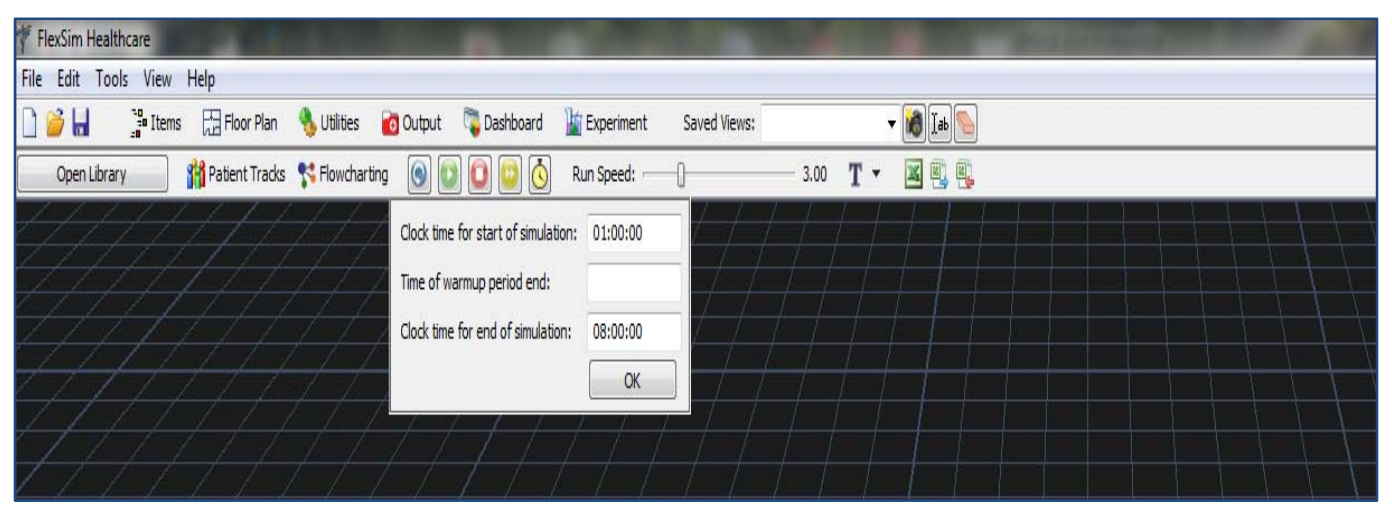

Figure 3.7 Specifying a Warm-Up Period in FlexSim

\subsection{Simio}

Developed in 2006 by Dennis Pegden, Arena users can easily adopt Simio. The major difference is that Simio is a $3 \mathrm{D}$ object-based modeling environment simulation package which is written in a C\# and .NET environment (http://www.simio.com/products/ and

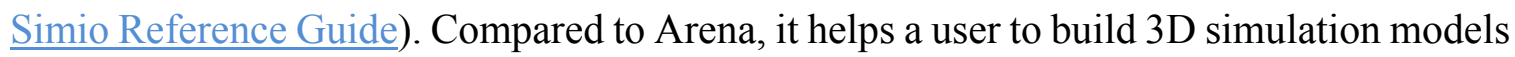
easily. Its 3D library is directly linked with Google Warehouse and allows any relevant 3D symbols to be added in a simulation model. 
Most features in Arena also are available in Simio and, as shown in Figure 3.8, a warmup period is implemented in "Experiments" after building a model. The Experiment Properties asks a user to determine a warm-up period as well as a confidence level. To access this option, the user can select the "Navigation window" first and then choose "Experiments". To specify the warm-up period, Simio provides related properties of the "Experiment" in Table 3.1.

\begin{tabular}{|c|c|c|c|}
\hline \multicolumn{3}{|c|}{ Browse } & $>$ \\
\hline \multicolumn{4}{|c|}{ Navigation: ModelWVExeriment1 } \\
\hline \multicolumn{3}{|c|}{ 1]. WarehouseExample } & $\Delta$ \\
\hline \multicolumn{3}{|c|}{$P$ ModelEntity } & $\equiv$ \\
\hline \multicolumn{3}{|c|}{ Model } & \\
\hline \multicolumn{3}{|c|}{ [1] Experiments } & \\
\hline \multicolumn{3}{|c|}{$\equiv$ Experiment1 } & \\
\hline \multicolumn{2}{|r|}{ MyServer } & & $\nabla$ \\
\hline \multicolumn{4}{|c|}{ Properties: Experiment1 (Experiment) } \\
\hline \multirow[t]{8}{*}{$\Xi$} & \multicolumn{3}{|l|}{ Analysis } \\
\hline & $\Xi$ Warm-up Period & 0 & \\
\hline & Units & Hours & \\
\hline & Default Replications & 10 & \\
\hline & Confidence Level & $95 \%$ & \\
\hline & Upper Percentile & $75 \%$ & \\
\hline & Lower Percentile & $25 \%$ & \\
\hline & Primary Response & & \\
\hline \multirow{3}{*}{$\begin{array}{l}\oplus \\
\square\end{array}$} & Advanced Options & & \\
\hline & General & & \\
\hline & Name & Experiment1 & \\
\hline
\end{tabular}

Figure 3.8 Specifying a Warm-Up Period in Simio 
Table 3.1 Properties of Warm-Up Period Control in Simio

\begin{tabular}{|c|c|c|}
\hline Property & $\begin{array}{l}\text { Valid } \\
\text { Entry }\end{array}$ & Description \\
\hline $\begin{array}{l}\text { Warm-up } \\
\text { Period }\end{array}$ & Real & $\begin{array}{l}\text { By default, Simio removes the information before a warm-up } \\
\text { period in order to obtain an estimate unaffected by an initial } \\
\text { condition. }\end{array}$ \\
\hline $\begin{array}{l}\text { Default } \\
\text { Replications }\end{array}$ & Integer & $\begin{array}{l}\text { This feature is useful to obtain results from multiple simulation } \\
\text { runs. It can specify the different replication numbers of each } \\
\text { different scenario. If a user does not change any value, the } \\
\text { default replication is } 1 \text {. }\end{array}$ \\
\hline $\begin{array}{l}\text { Confidence } \\
\text { Level }\end{array}$ & $\begin{array}{l}90 \% \\
95 \% \\
98 \% \\
99 \%\end{array}$ & $\begin{array}{l}\text { After running multiple replications, Simio will calculate } \\
\text { confidence interval of half-width statistics of average results } \\
\text { across replications. }\end{array}$ \\
\hline
\end{tabular}

\subsection{ExtendSim}

ExtendSim (http://www.extendsim.com/) has an open structure to help users modify its library (Banks, 1998), a feature which attracts us to focus on this package in implementation of an automatic MSER calculation. Additionally, the syntax of its programming language, ModL, is very similar to $\mathrm{C}$, and allows the modeler to save output data in memory instead of a hard disk, facilitating faster computation time. As shown in Figure 3.9, a warm-up period can be implemented in ExtendSim using the "Clear Statistics" option in the statistics library.

\subsection{SimCAD}

SimCAD (https://www.createasoft.com/) is a DES environment that provides userfriendly features to create a model. It advertises that even simulation novices can build a model without spending a long time to figure it out. However, it appears that a user tends to follow built-in functions exclusively. Even though it can foster model building, some features do not reflect the notion of statistics or methodologies from systems engineering. 


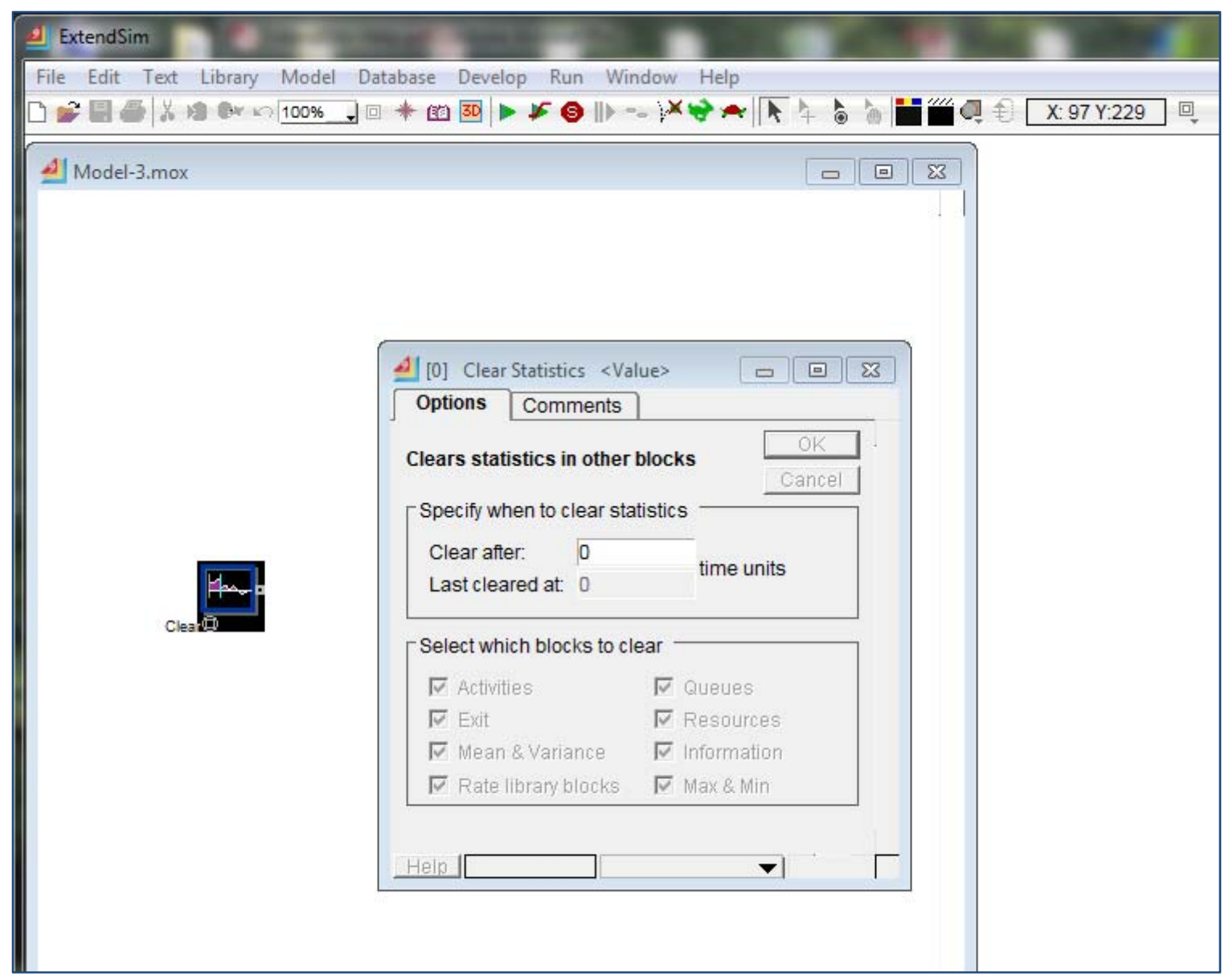

Figure 3.9 Warm-Up Period in ExtendSim using Clear Statistics under Statistics library

Furthermore, it does not clearly include a warm-up period as a basic option. We observed that an application specialist in SimCAD did not know how to handle a warm-up period. When we had a later conversation with a lead development engineer, he suggested how to achieve the same functionality of a warm-up period. Apparently, it can deal with the warmup period, but requires the end user to write additional code. The SimCAD GUI is shown in Figure 3.10. 


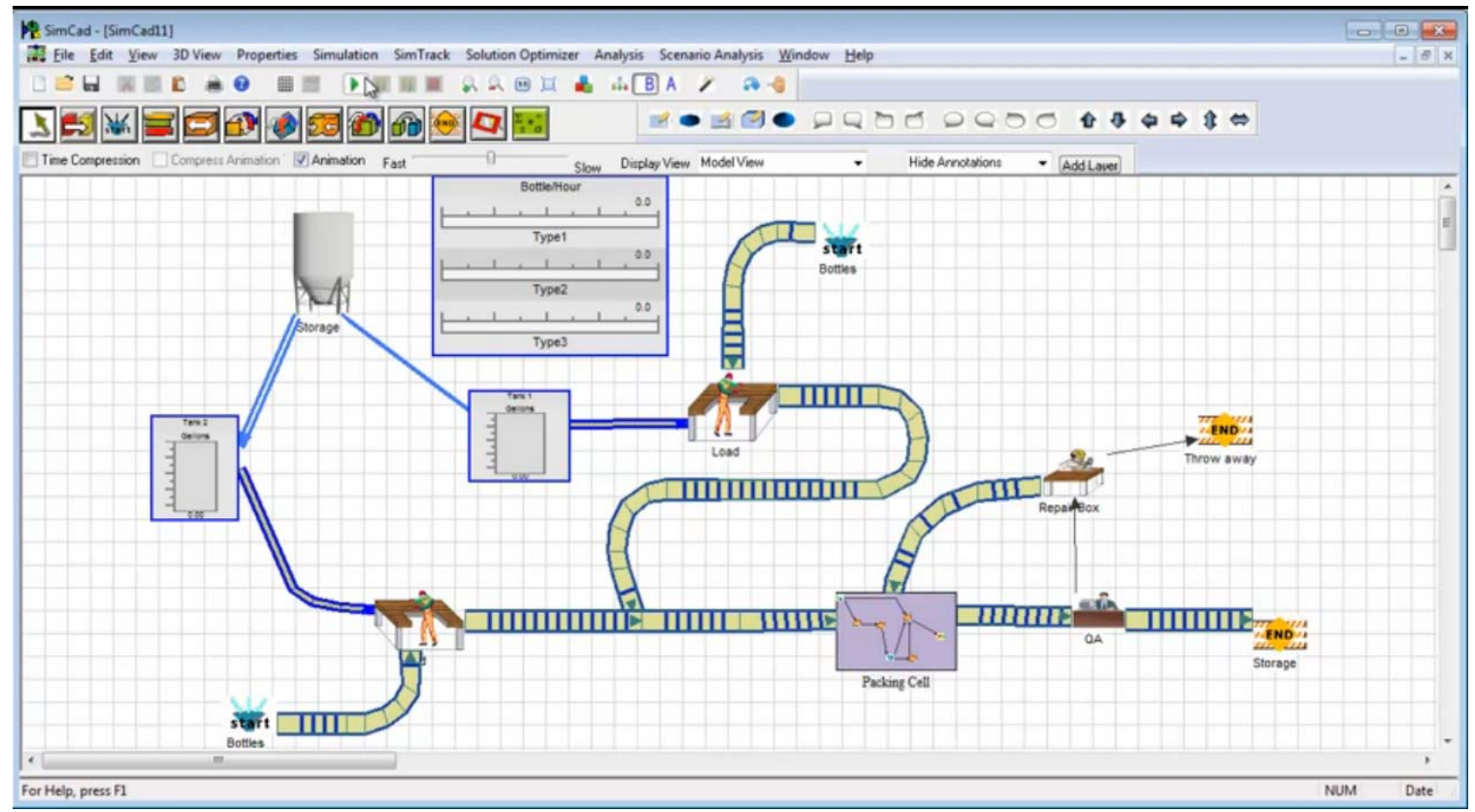

Figure 3.10 GUI of SimCAD 


\subsection{Post analysis}

Heretofore MSER logic has been implemented by individuals in custom-built, datadriven postprocessors. In contrast to the Arena and ExtendSim online implementations provided in Chapter 4, these codes operate on output sequences generated by prior simulation runs, rather than continuously while simulating a model. Law (2015) provides a notable example in his test applications of MSER. In these examples, Law imported simulation output to an Excel spreadsheet developed by Katy Hoad of the University of Warwick. This spreadsheet incorporates a VBA macro to perform all MSER calculations.

To promote more widespread adoption of MSER, we developed MSER codes in several popular programming languages. These codes are given in Chapter 6 and also are available online at the MSER Laboratory. Each code required us to implement logic using alternate programming syntax and built-in functions. While run times varied on test cases, all of the applications yielded identical results and the identical cases, as expected.

Five different applications were written: two in the open-source code applications, $\mathrm{R}$ and $\mathrm{C} / \mathrm{C}++$, and three in the proprietary software applications, Matlab, SAS, and VBA. Each implementation has its own strength of computing an optimal truncation point by using the concept of an array, which makes these distinct from each other. Once we understand the common workflow to calculate MSER, the difference in what language it is written is minor. That is, we just need to know and exchange specific built-in functions inside each and follow language specific syntax. Using these built-in functions may or may not be efficient computationally. If the data sets are not large, however, the functions tend to work better than using user-defined functions based on loop and conditional statements. 
An overview of these computing languages is provided in the following section. We describe each pertinent programming language or application to implement and run MSER so it is useful to illustrate key features before accounting for syntax in different codes. The following part will explain the history, background, and features from each programming language.

\subsubsection{R}

$\mathrm{R}$ is known as a statistical computing language that supports publication-quality graphics. Based on the S language, R is part of the GNU freeware project, an open sourceprogramming environment. Because it is freeware, more academic institutions tend to use $\mathrm{R}$ rather than commercial alternatives. In addition, $\mathrm{R}$ can run on a wide variety of popular operating systems such as Windows, Linux, and MacOS. User contributions to R enrich its functionalities along with research development and it is very flexible to interact with other languages such as $\mathrm{C}, \mathrm{C}++$, and FORTAN. Source: What is R?: Introduction to $\mathrm{R}$ (http://www.r-project.org/)

\subsubsection{C/C++}

Dennis Ritchie created C in 1972 at Bell Laboratories and Bjarne Stroustrup developed $\mathrm{C}++$ in the early 1980s, also at Bell Laboratories. Among the programming languages discussed here, $\mathrm{C} / \mathrm{C}++$ are the only languages to compile the code before running the logic. Generally speaking, $\mathrm{C}++$ is superset of $\mathrm{C}$. Thus codes written in $\mathrm{C}$ can usually be transported to $\mathrm{C}++($ some exceptions exist). Both $\mathrm{C}$ and $\mathrm{C}++$ demand very rigorous and strict coding, but are very fast to execute (Prata, 2003 and 2005). 


\subsubsection{Matlab}

As the name suggests, Matlab is useful to manipulate matrix operations for numerical computations and visual representations. Matlab is intended to support technical computing. It advertises that the mastery of its language, tools, and built-in functions can help users obtain the results efficiently. We also witness that more and more engineers and students areadopting this technical language. Source: Matlab primer (http://www.mathworks.com/help/pdf_doc/matlab/getstart.pdf)

\subsubsection{SAS}

SAS is proprietary software to solve problems from real business to academic research. Its exemplar tasks include file IO (data entry, retrieval, and management), ODS (output delivery system for presentation of report and pertinent graphics), statistical and mathematical analysis, and functionality for operations management/research problems such as business planning, forecasting, decision, and others.

To perform MSER calculation, we use a specific module to support array and matrix manipulation, SAS/IML (Interactive Matrix Language). This is a complete programming language with a dynamic, interactive environment for programmers, statisticians, researchers, and high-end analysts. After obtaining data or processed information, this interactive language is designated for more complex and sophisticated analysis to explore target data sets. Its user interface is similar to SAS, as well as Matlab. However, creating one's own SAS/IML modules becomes much easier than using SAS by itself. Every 
application can be run either interactively or in batch. Furthermore, it can adopt R code via the IML server. Source: SAS 9.3 Language Reference (http://support.sas.com/documentation/cdl/en/lrcon/65287/PDF/default/lrcon.pdf) and SAS/IML fact sheet (http://www.sas.com/resources/factsheet/sas-iml-factsheet.pdf)

\subsubsection{VBA}

Excel, the spreadsheet included in MS Office, is equipped with the Visual Basic for Applications (VBA) language. Whenever extra analysis or calculation cannot be performed by built-in functions, VBA is the last source to count on. VBA is based on the Basic programming language and relatively easy to learn. However, its speed of execution is sacrificed for the stake of simplicity. VBA also supports an array format and its dynamic properties can be used for the development of MSER inside Excel. When a user opens the Excel workbook, the user can open Visual Basic Editor on the Developer Tab and build various functions to meet the objectives of a modeler.

\subsection{Merits of the alternative codes}

In summary, needless to say, the application written in $\mathrm{C} / \mathrm{C}++$ is more error prone and time consuming because we can only depend on their basic math library. In addition to this effort, we have to define types of all variables with care. However, it is cost effective and very fast to process long time series, which is a general benefit in high-level programming language. VBA is a more user-friendly interface to interact with end users, but demands more time to compute MSER statistics. We acknowledge that there might be room to optimize code performance in Visual Basic as we just use VBA to compute MSER. Three 
other applications will help whoever has some expertise or experiences among R, Matlab, and SAS to be familiar with MSER logic. These three applications develop their own array manipulation tools and use built-in functions to code MSER logic in simple ways. As long as the user knows and uses the right functions, it would reduce the time and effort to write applications with these. 


\section{Chapter 4. The MSER Laboratory}

Ideally, simulationists from industry and the academy collaborate to keep abreast of developments reported in the simulation literature. Commercial simulation software continues to incorporate new research findings, but typically lags in the pace of adoption because of the considerable investment required to revise current codes or to add new logic in a current version of software. One of the main goals of this research is to spur the implementation of MSER in commercial software and practical application.

To this end we have created a web-based laboratory that is an open and accessible resource for those who are interested in improving, applying, and extending the use of MSER. Included in the MSER Laboratory are (1) an archive of key research articles, (2) a repository of MSER codes that may be freely downloaded, and (3) a set of concrete and user-friendly examples that illustrate the application of these codes. The MSER Laboratory is hosted at the University of Virginia at http://faculty.virginia.edu/MSER/.

We have observed that increasingly researchers are publishing their sample data sets as well as their codes. However, it is not an easy task to decipher code written in a language with which one is not familiar. That is why we facilitate the implementation of MSER in well-known simulation software such as ExtendSim, Arena, and ProModel/MedModel (see Chapter 5), as well provide MSER postprocessors in written in R, SAS, Matlab, VBA, and $\mathrm{C} / \mathrm{C}++$ (see Chapter 6$)$. We seek continuity of this beginning by maintaining an up-to-date 
development in MSER from those who make progress. Furthermore, we appreciate receiving any constructive comments to improve the quality of this site.

In this chapter we take a very brief guided tour through screen shots of the Lab as it currently is configured, while additional details are provided in later chapters. The content in this site gives the general idea of MSER (Figure 4.1), its history related to research articles (Figure 4.2), sample codes as well as sample test sets (Figure 4.3) and the basic math derivation of the MSER statistic (Figure 4.4).

These implementations in commercial simulation software are distinct from the current, arbitrary warm-up determination because these:

- Determine a truncation point automatically

- Minimize unnecessary user input

- Provide figures and tables to support this determination

All information is downloadable by any interested users and the site will help researchers exchange and update any new developments. 


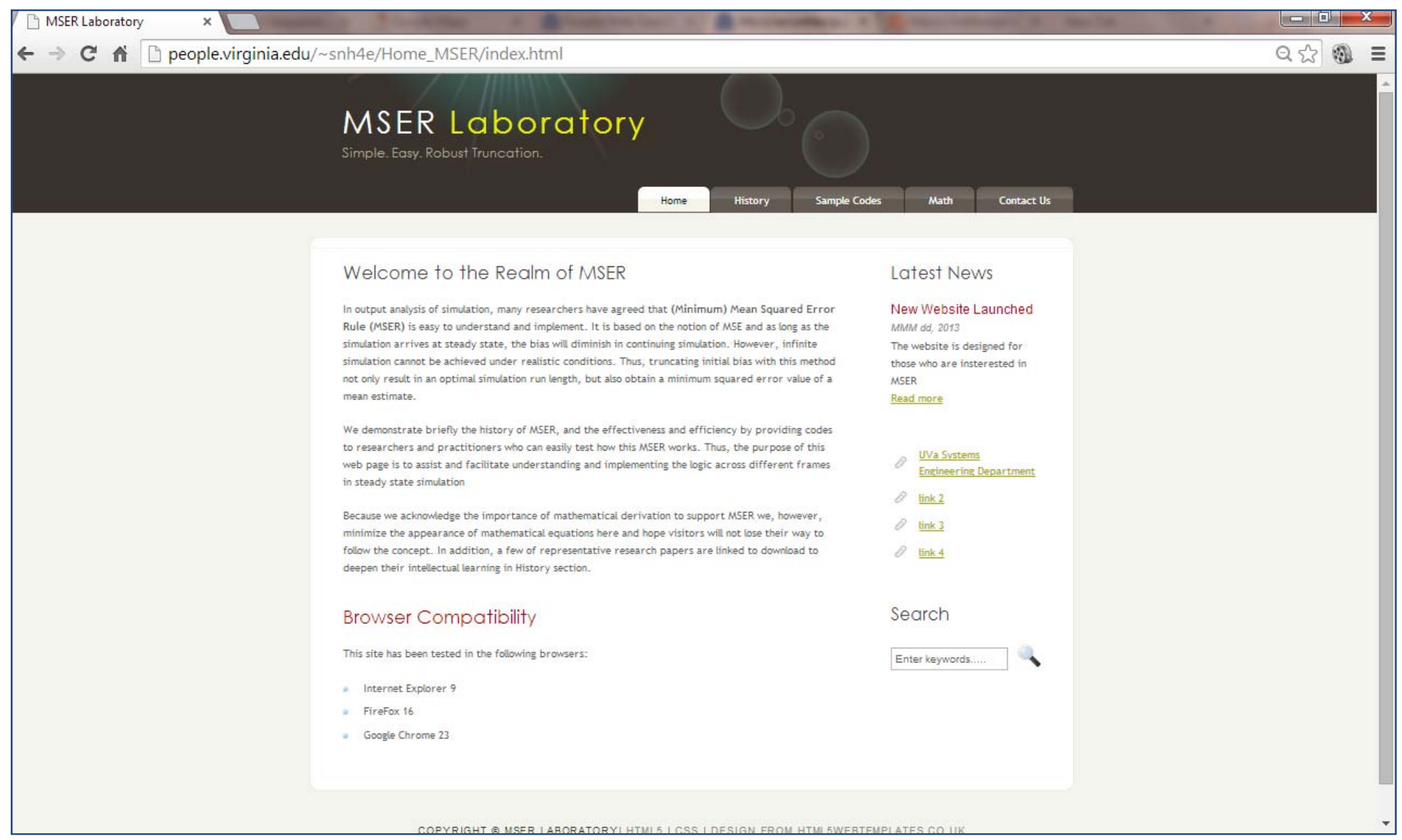

Figure 4.1. Introduction of MSER Laboratory Web page 


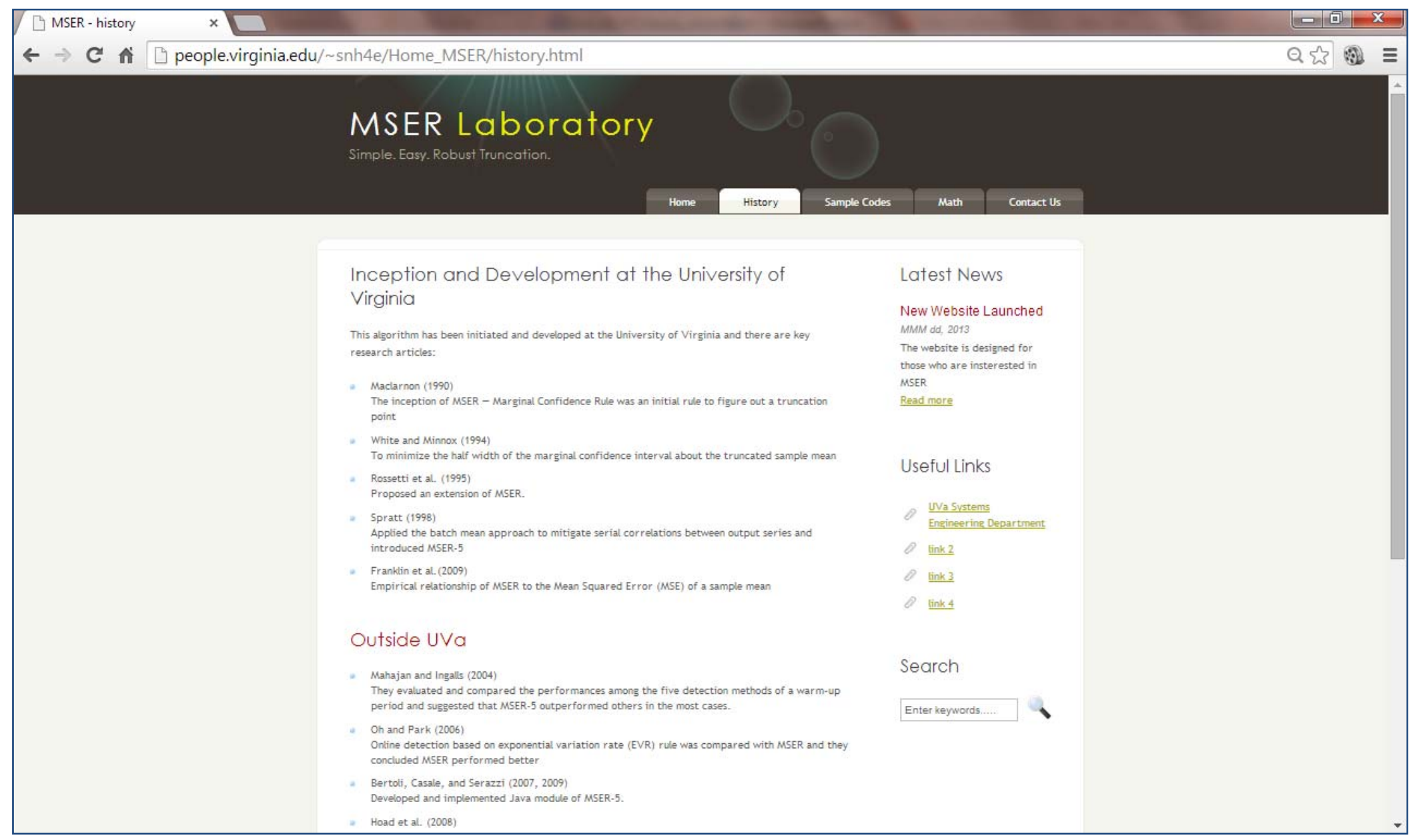

Figure 4.2. History of MSER Laboratory Web page 


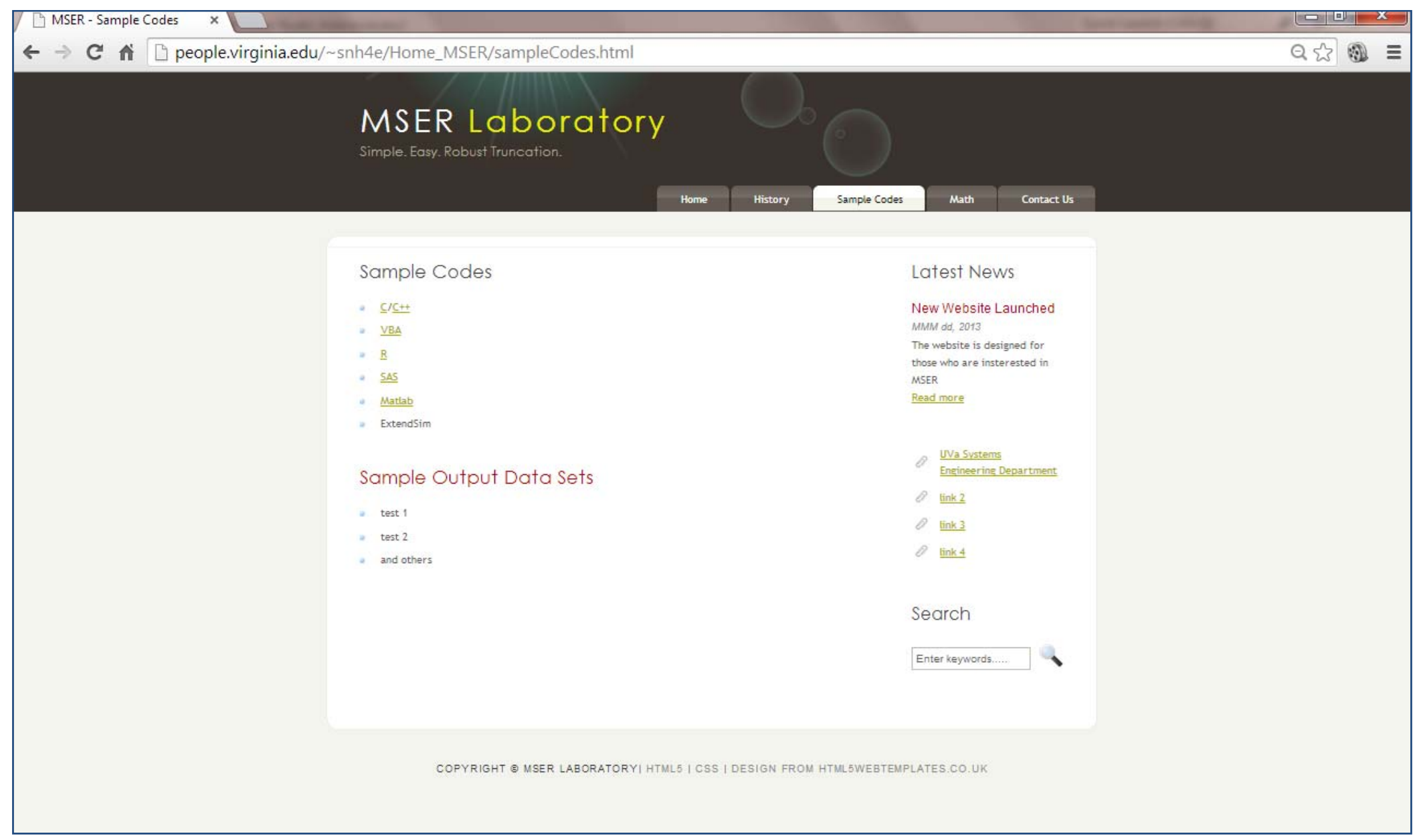

Figure 4.3. Sample Codes of MSER Laboratory Web page 


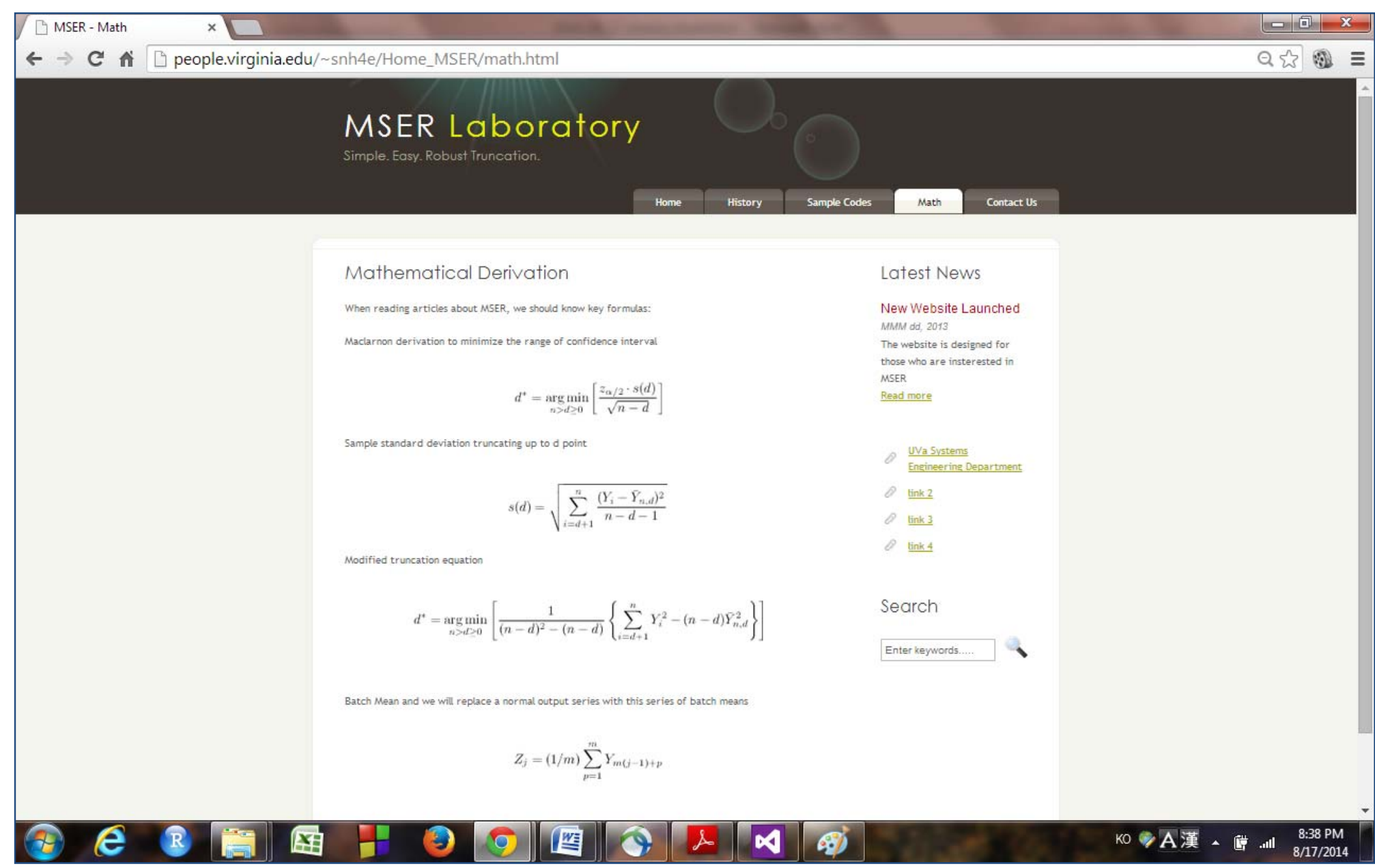

Figure 4.4. Math of MSER Laboratory Web page 


\section{Chapter 5. Implementation of MSER in Commercial Software}

Before selecting appropriate simulation software suites, we must check whether the software package can update the MSER-statistic in a designated way while it saves the output to memory. After reviewing current discrete-event simulation software (see Chapter 3), we chose three representative software packages - ExtendSim, Arena, and ProModel— with which we can calculate the MSER statistic online. In this Chapter we present examples of the incorporation of MSER logic in the model process flow diagrams, together with the corresponding source code or module structure needed to support implementation for each of these languages.

\subsection{ExtendSim Implementation}

ExtendSim (originally named "Extend"), from Imagine That, Inc., is a general-purpose software suite for continuous, discrete-event, and hybrid simulation (Banks 1998, Krahl 2012). After we confirmed its capability to store output data sets, we collaborated with Dave Krahl at Imagine That and appreciated his efforts and time, even under his tight schedule, on the creation of a MSER library in ExtendSim. The MSER algorithm is implemented in ExtendSim's C-based ModL language. Only minor modifications relating to the user interface and variable initialization were required to convert the algorithm from ANSII C to ModL. The MSER block is fully integrated into ExtendSim and can be used in any ExtendSim model in the future. 
The library collects data continuously from any relevant blocks and is designed to compute a MSER statistic, a truncation point, and a mean estimate associated with the truncation point. Another feature in this library includes the function to set up the computing frequency via a user input. The default batch size is 5 and batch size can be varied via setting different dialog parameters.

\subsubsection{ExtendSim Process Flow}

Figure 6.1 depicts a tandem queue model in ExtendSim, with the MSER module included to collect statics on the waiting time in Queue 2 tied with the GUI for the MSER calculator.

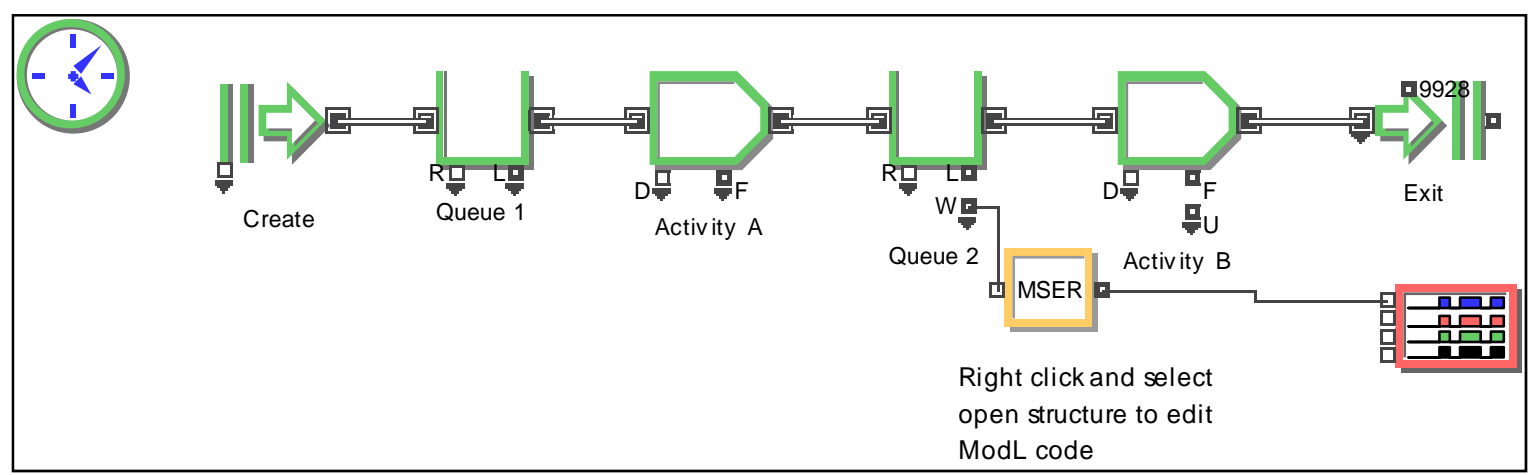




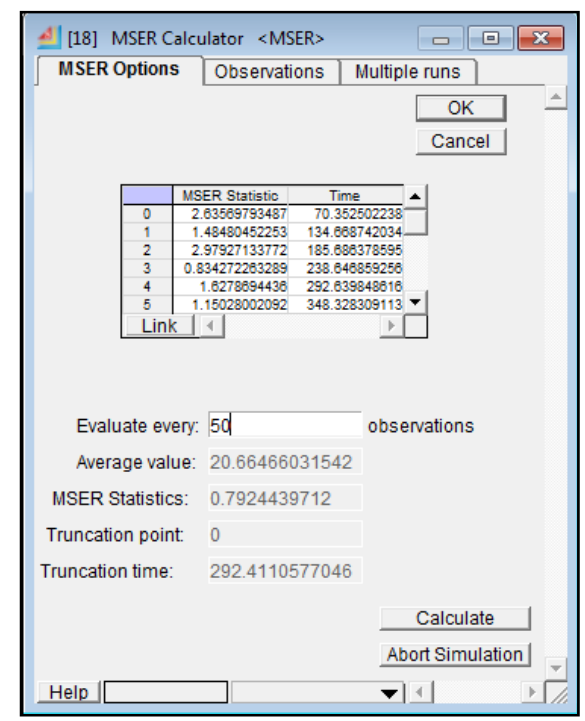

Figure 5.1 MSER Implementation and GUI in ExtendSim

\subsubsection{ExtendSim Code}

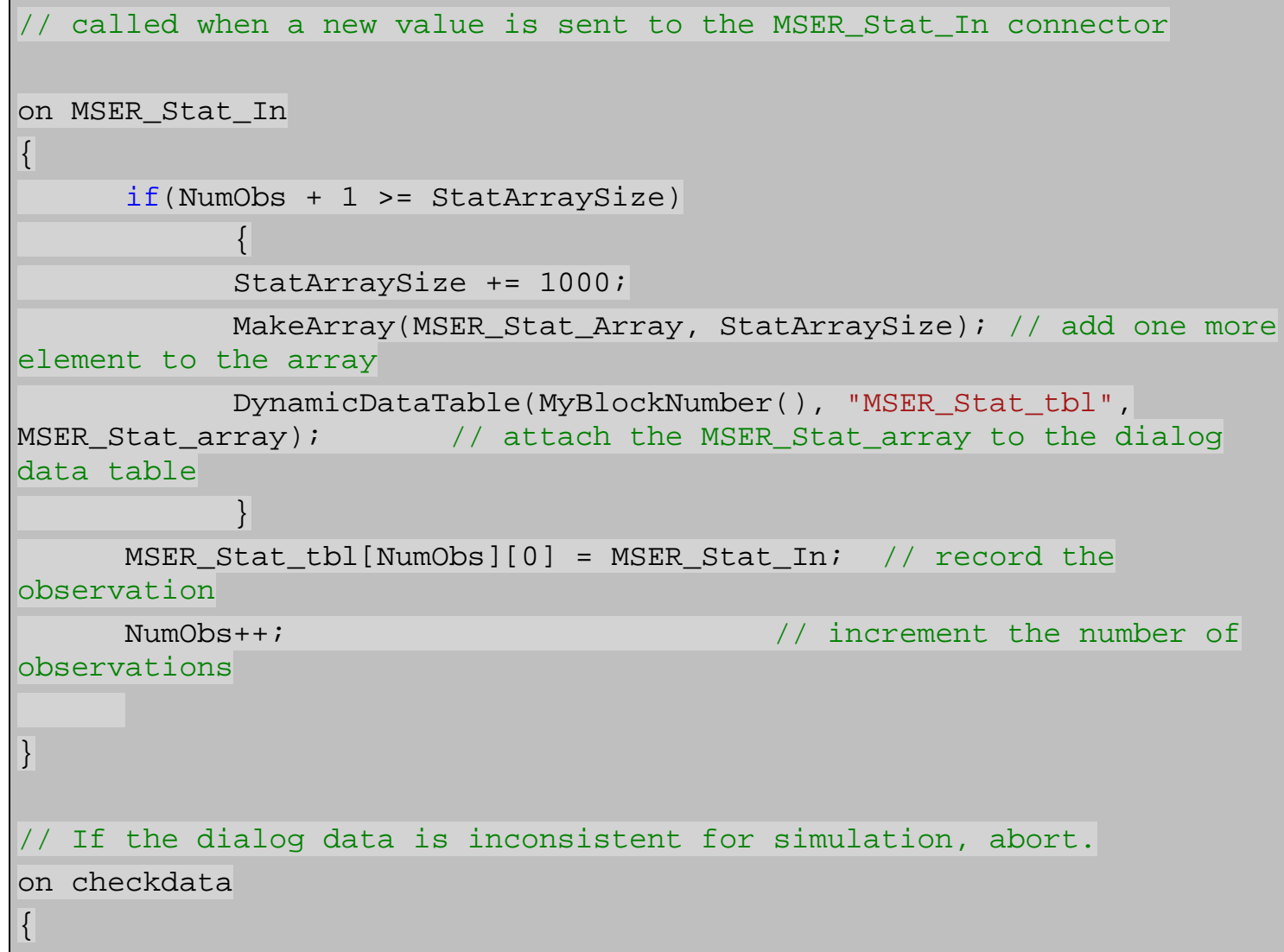




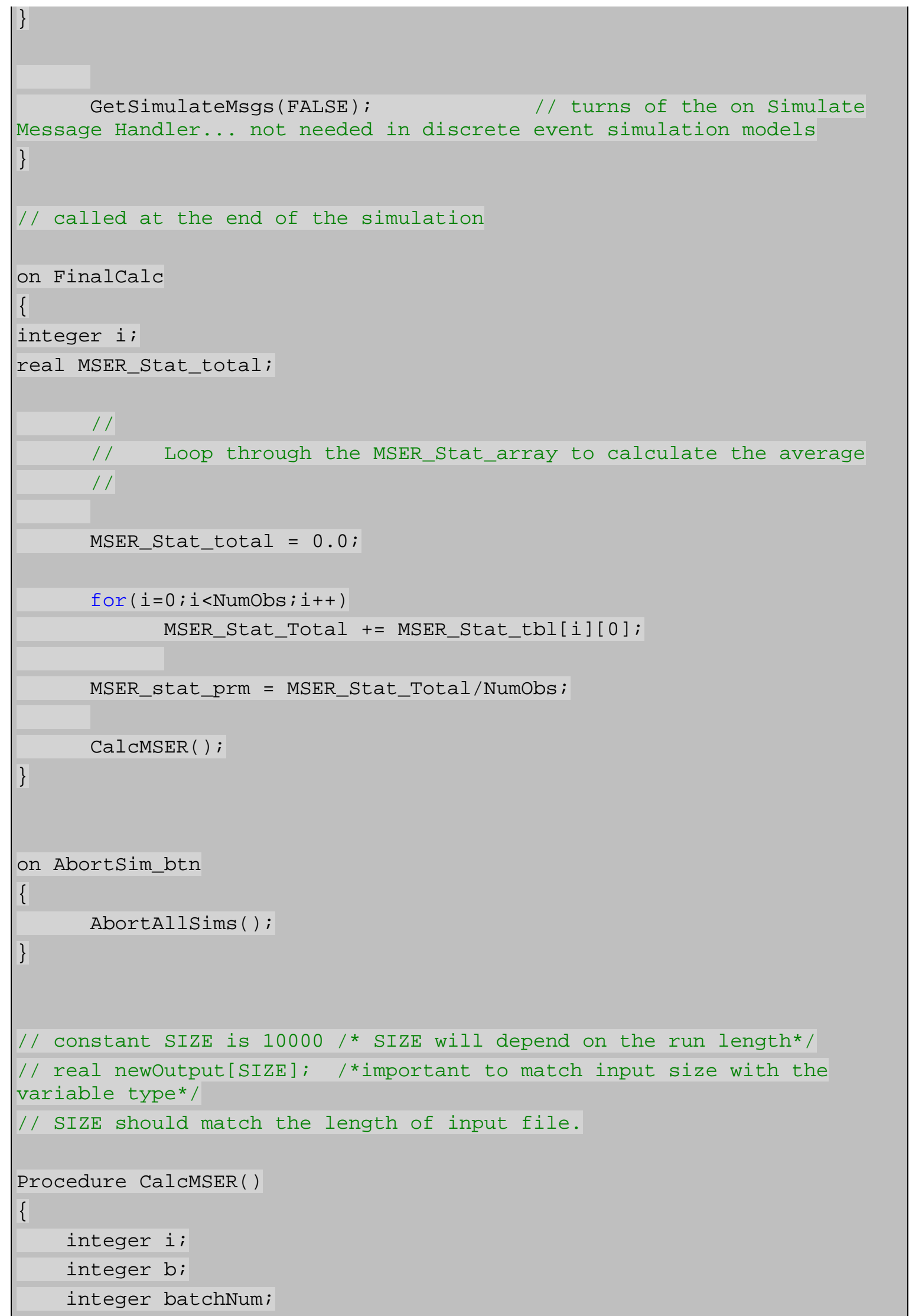




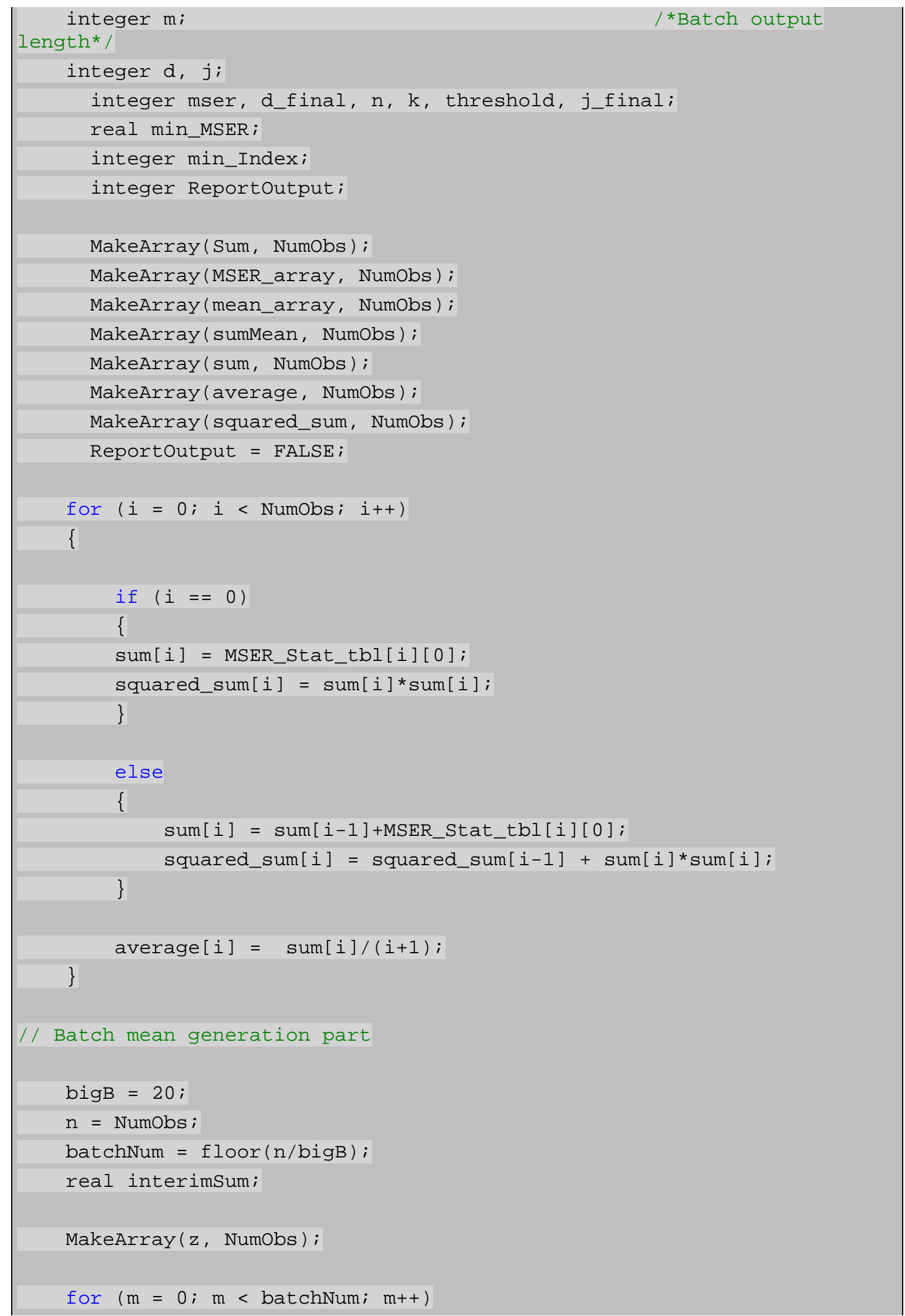




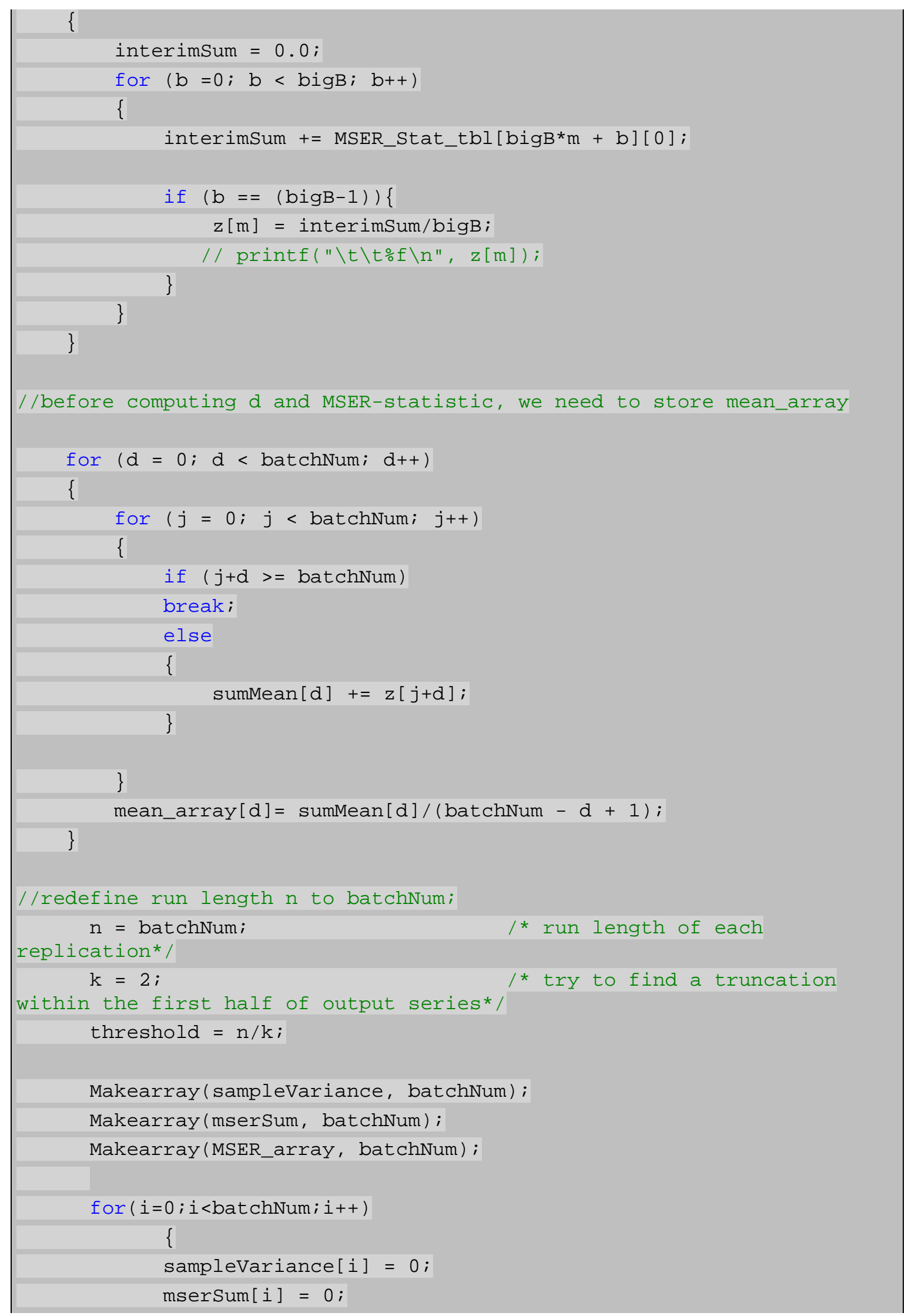




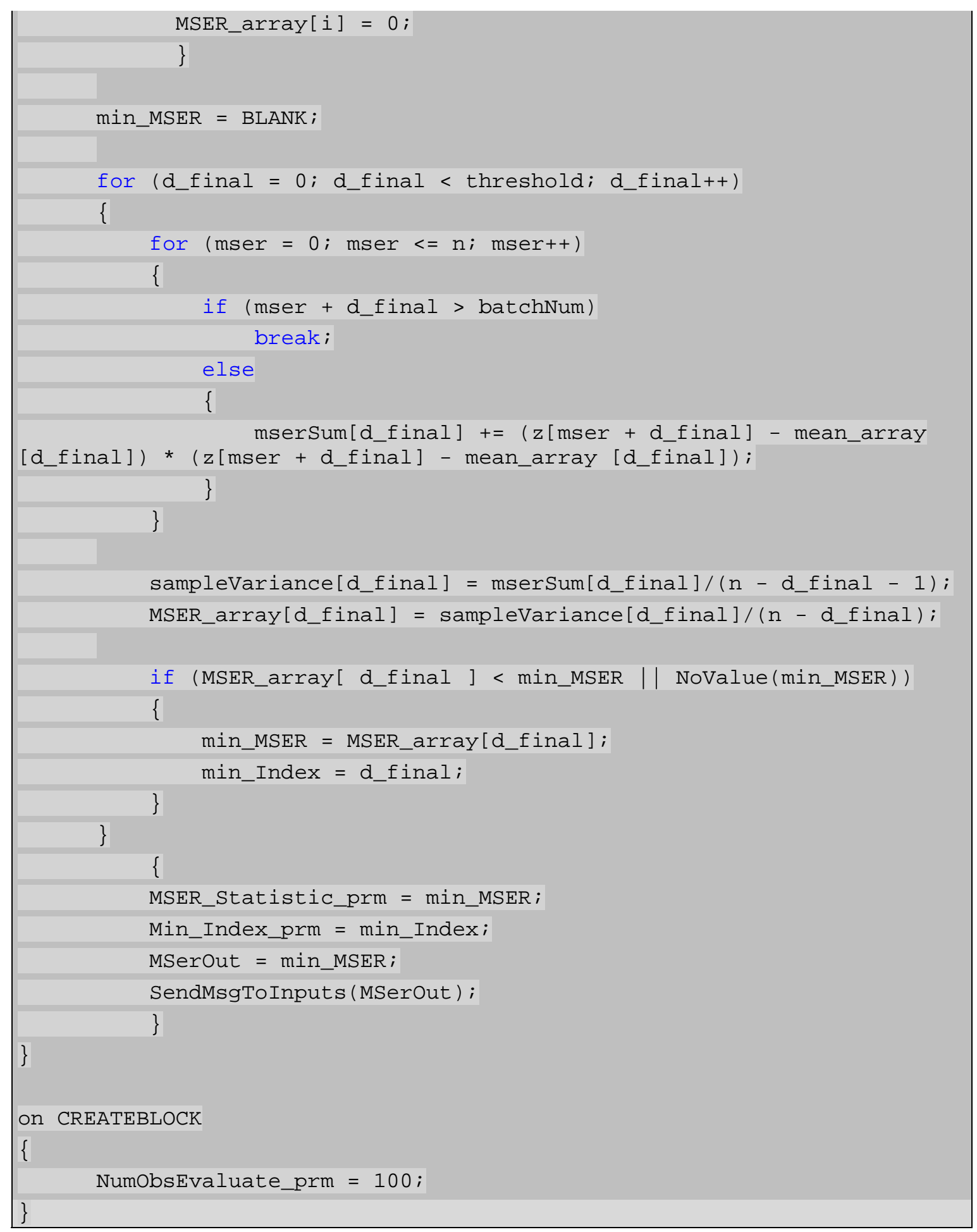

\subsection{Arena Submodel Implementation}


Although it is desirable to develop MSER as a built-in function in Arena, as has done in ExtendSim, we have as yet been unable to persuade Rockwell Software to invest in this functionality. As an immediate alternative, we chose to implement the MSER calculation logic inside an Arena submodel (Kelton, et al. 2010). This submodel is generic and can be inserted in any Arena project. The MSER-optimal point can be calculated for any output by assigning this attribute as the input to the MSER submodel.

\subsubsection{Arena Process Flow}

Figure 5.2 depicts an M/M/1 queue in Arena with a MSER submodel included to collect statistics on the waiting time in queue defined in the "Service" process module. The wait time in queue is assigned to an attribute before the corresponding entity enters the submodel named "MSER Module". In models more complex than this simple queue, we can add copies of the submodel to compute MSER statistics for multiple attributes or variables anywhere in the process flow. For instance, if a researcher wants to compute the wait time in both queues in a tandem queue model, she/he needs to include these two submodels, one for the output of each service process.

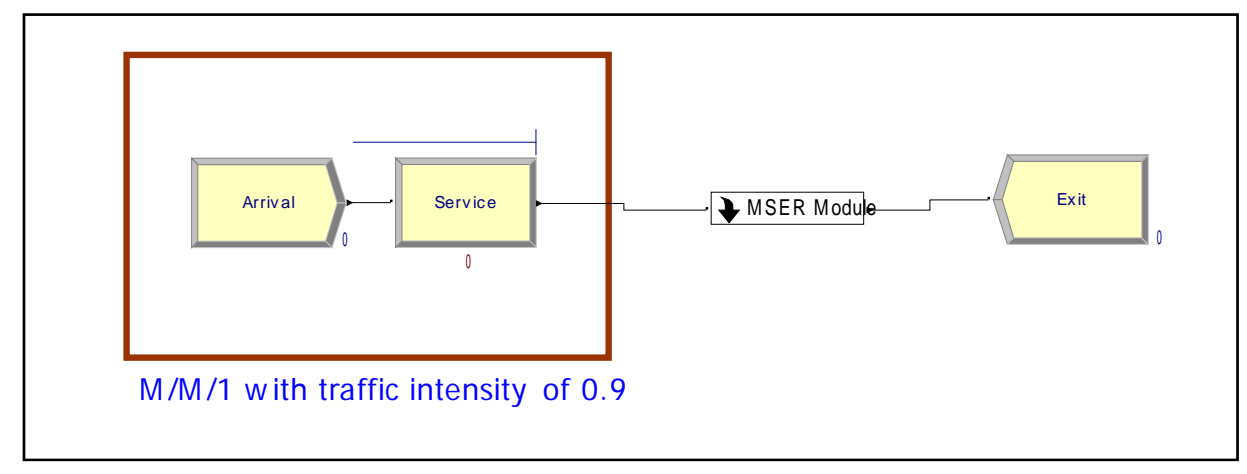

Figure 5.2 Main model of an M/M/1 queue in Arena with the MSER module included to collect statistics on the waiting time in Service queue 


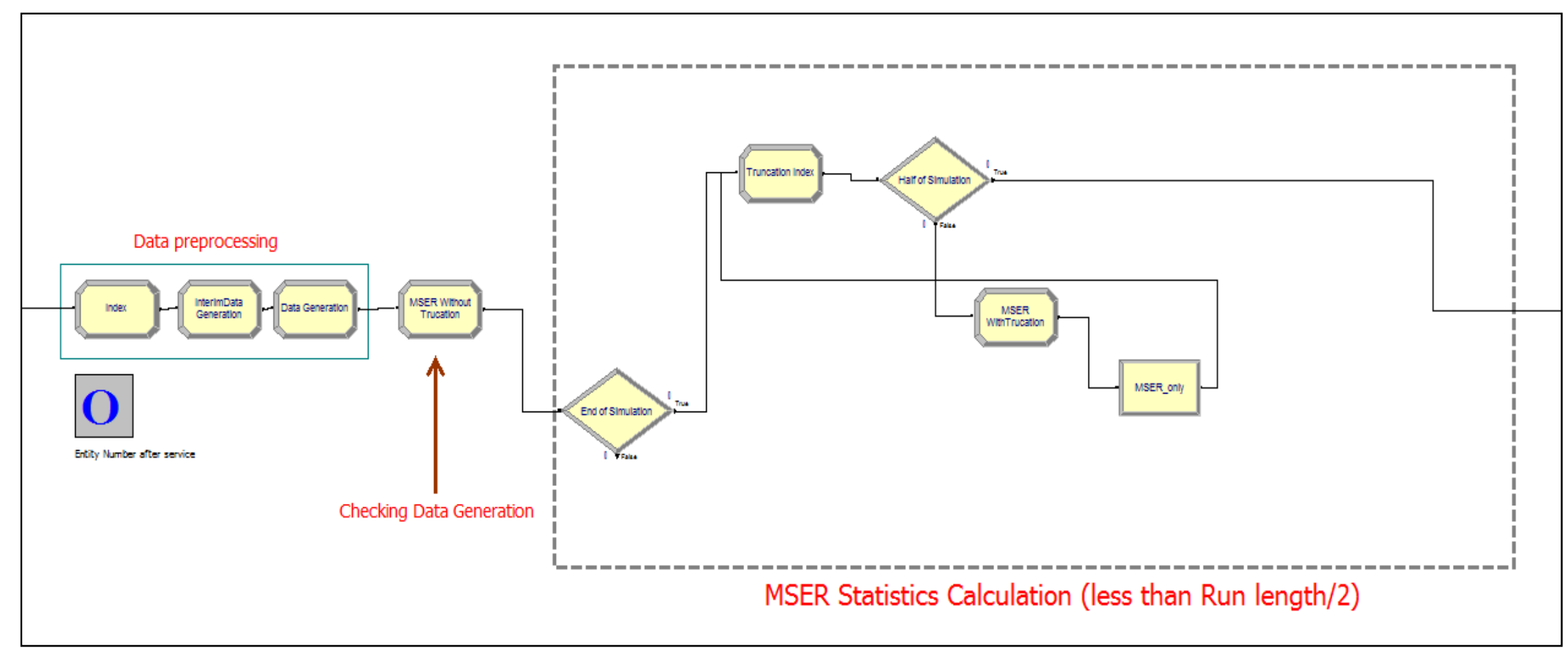

Figure 5.3 Details of the MSER calculation in the Arena 


\subsubsection{Arena MSER Modules}

Figure 5.3 shows the Arena process flow that implements the MSER computation. Table 5.1 lists the global and local variables and arrays used by the submodel. The User's Guide in Appendix I provides more details and a fully worked-out example.

Table 5.1. Global, Local variables and Arrays

\begin{tabular}{|c|c|c|c|}
\hline No. & Variable (array) name & Location of block & Usage \\
\hline 1 & v_Counter & Index & $\begin{array}{l}\text { Record entity number } \\
\text { v_Counter }+1\end{array}$ \\
\hline 2 & v_X & $\begin{array}{l}\text { InterimData } \\
\text { Generation }\end{array}$ & Record entity wait time \\
\hline 3 & $\mathrm{v} \_\mathrm{X} 2$ & $\begin{array}{l}\text { InterimData } \\
\text { Generation }\end{array}$ & Record (entity waiting time) ${ }^{2}$ \\
\hline 4 & v_inter_cumX & $\begin{array}{l}\text { InterimData } \\
\text { Generation }\end{array}$ & Holder for summation of $\mathrm{v} \_\mathrm{X}$ \\
\hline 5 & $\mathrm{v}$ _inter_cumX2 & InterimData & Holder for summation of $\mathrm{v} \_\mathrm{X} 2$ \\
\hline 6 & v_Mean & DataGeneration & v_cumX(v_Counter) $/ v_{\text {v_Counter }}$ \\
\hline 7 & $v_{\text {_ }}$ cumX & DataGeneration & v_inter_cumX(v_counter $)$ \\
\hline 8 & $\mathrm{v}_{-}$cumX2 & DataGeneration & $\mathrm{v}$ _inter_cumX2(v_counter) \\
\hline 9 & v_MSER_test & $\begin{array}{ll}\text { MSER } & \text { Without } \\
\text { Trucation } & \end{array}$ & $\begin{array}{l}\text { (v_cumX2(v_Counter) - v_Counter * } \\
\text { v_Mean(v_Counter }) * \\
\left.\text { v_Mean }\left(v_{-} \text {_Counter }\right)\right) /\left(\left(v_{-} \text {_counter - }\right.\right. \\
\left.0) *\left(v_{-} \text {counter }-0\right)\right)\end{array}$ \\
\hline 10 & StopRule & $\begin{array}{l}\text { End of Simulation } \\
\text { (Decision block) }\end{array}$ & $\begin{array}{l}\text { Global variable to check the end of } \\
\text { simulation, } 10000 \text { that confirm } \\
\text { StopRule }=\text { v_Counter }\end{array}$ \\
\hline 11 & v_Truncation & Truncation Index & $\mathrm{v}_{\text {_Truncation }}+1$ \\
\hline 12 & v_MSER_final & MSER WithTruncation & 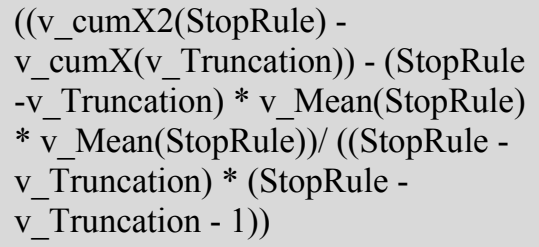 \\
\hline
\end{tabular}

\subsection{Promodel/Medmodel Implementation}

Promodel/Medmodel is equipped with a function call to use DLL so computing additional statistic can be feasible as mentioned previously. We test $\mathrm{M} / \mathrm{M} / 1$ with traffic 
intensity of 0.9. As shown in Figure 5.4, this simple model consists of three nodes representing entity arrival, processing, and exit, as well as a waiting queue for processing.

\subsubsection{Promodel/Medmodel Process Flow}

After compiling the DLL to compute MSER statistic, this file is listed in "External Files" as "DLL". It is saved in a working directory, or the model needs to be told the directory where it is compiled. $\mathrm{XSUB}()$ uses Med.dll to compute MSER the statistic aDuration that records time in system, as shown in Figure 5.5.

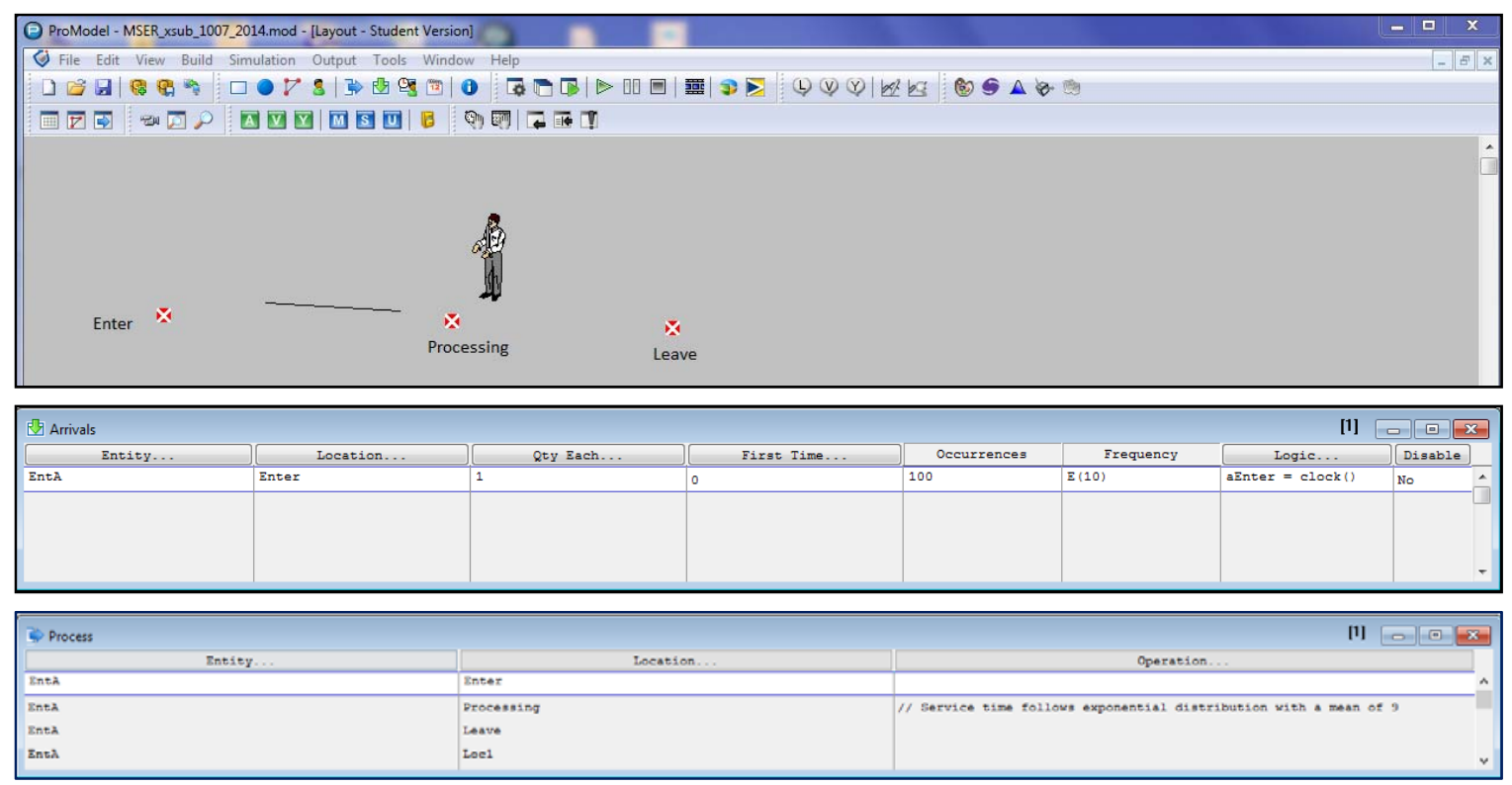

Figure 5.4 M/M/1 Model in ProModel

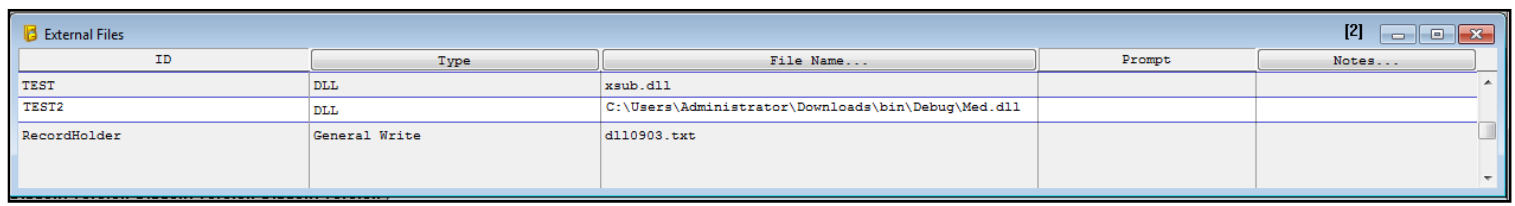

\begin{tabular}{|l|l|l|r|}
\hline Users > Administrator > Downloads > bin > Debug & & \\
\hline Name & Date modified & Type & Size \\
$\square$ libMed.dll.a & $9 / 3 / 20148: 20 \mathrm{PM}$ & A File & $2 \mathrm{~KB}$ \\
$\square$ libMed.dll.def & $9 / 3 / 20148: 20 \mathrm{PM}$ & DEF File & $1 \mathrm{~KB}$ \\
\hline O Med.dll & $9 / 3 / 20148: 20 \mathrm{PM}$ & Application extension & $25 \mathrm{~KB}$ \\
\hline
\end{tabular}




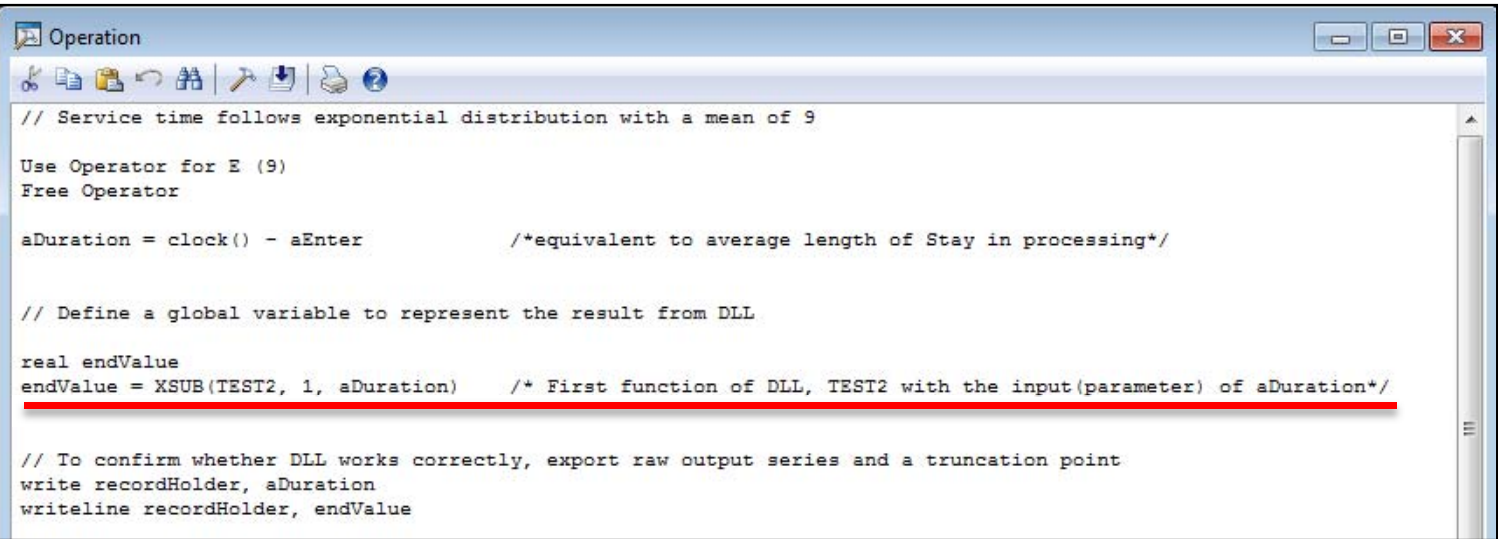

Figure 5.5 DLL Usage in ProModel

\subsubsection{ProModel DLL Code}

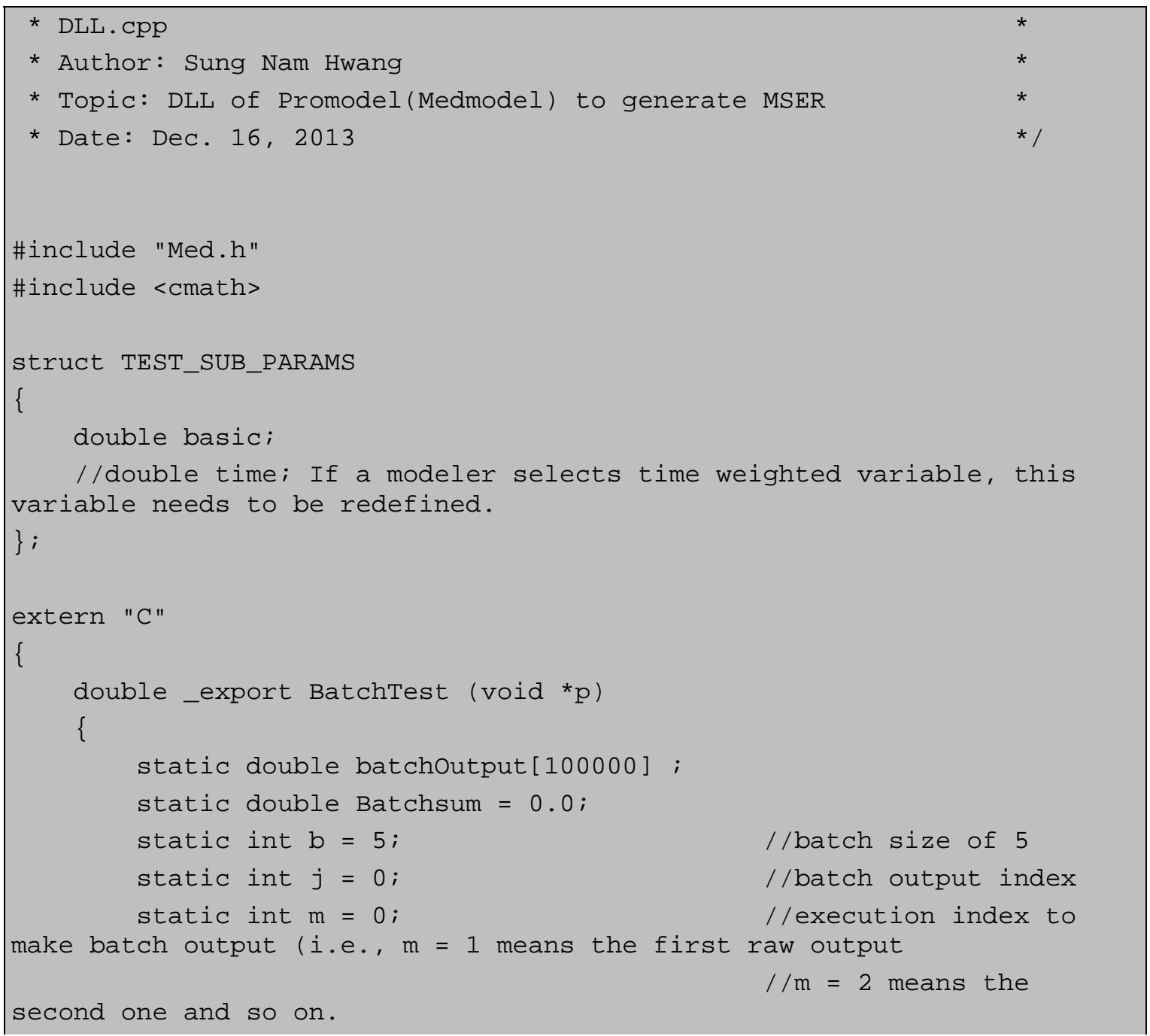




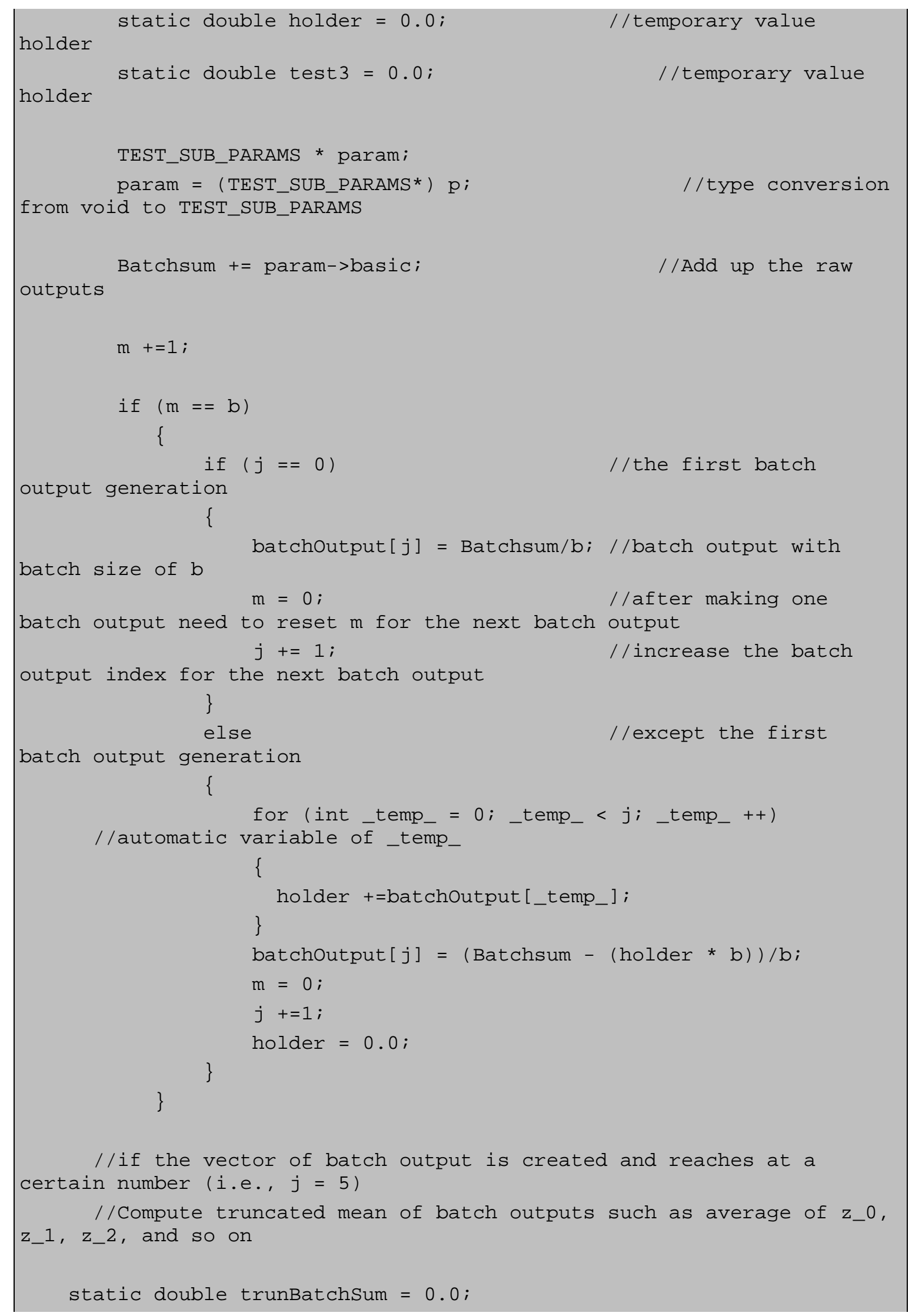




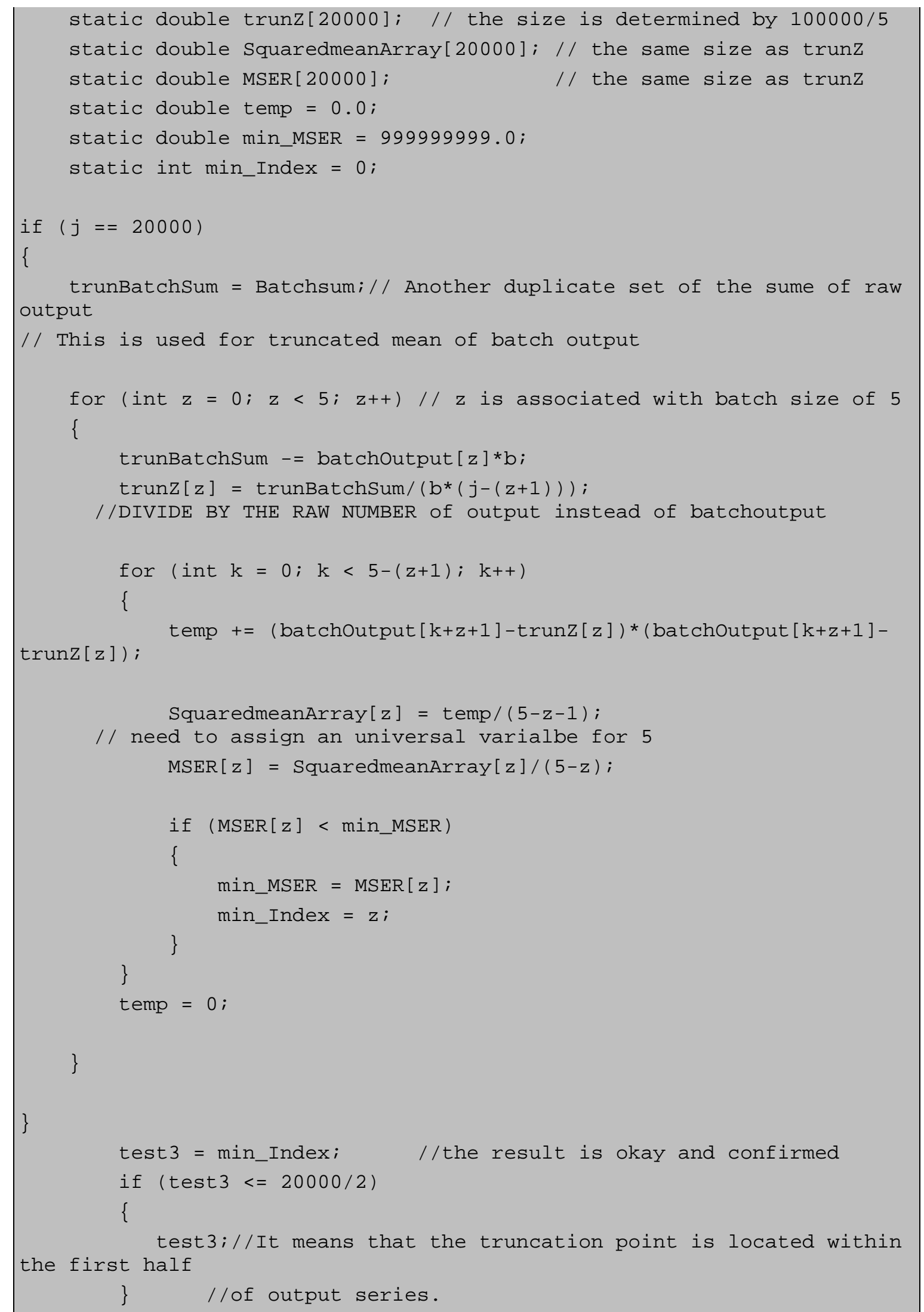




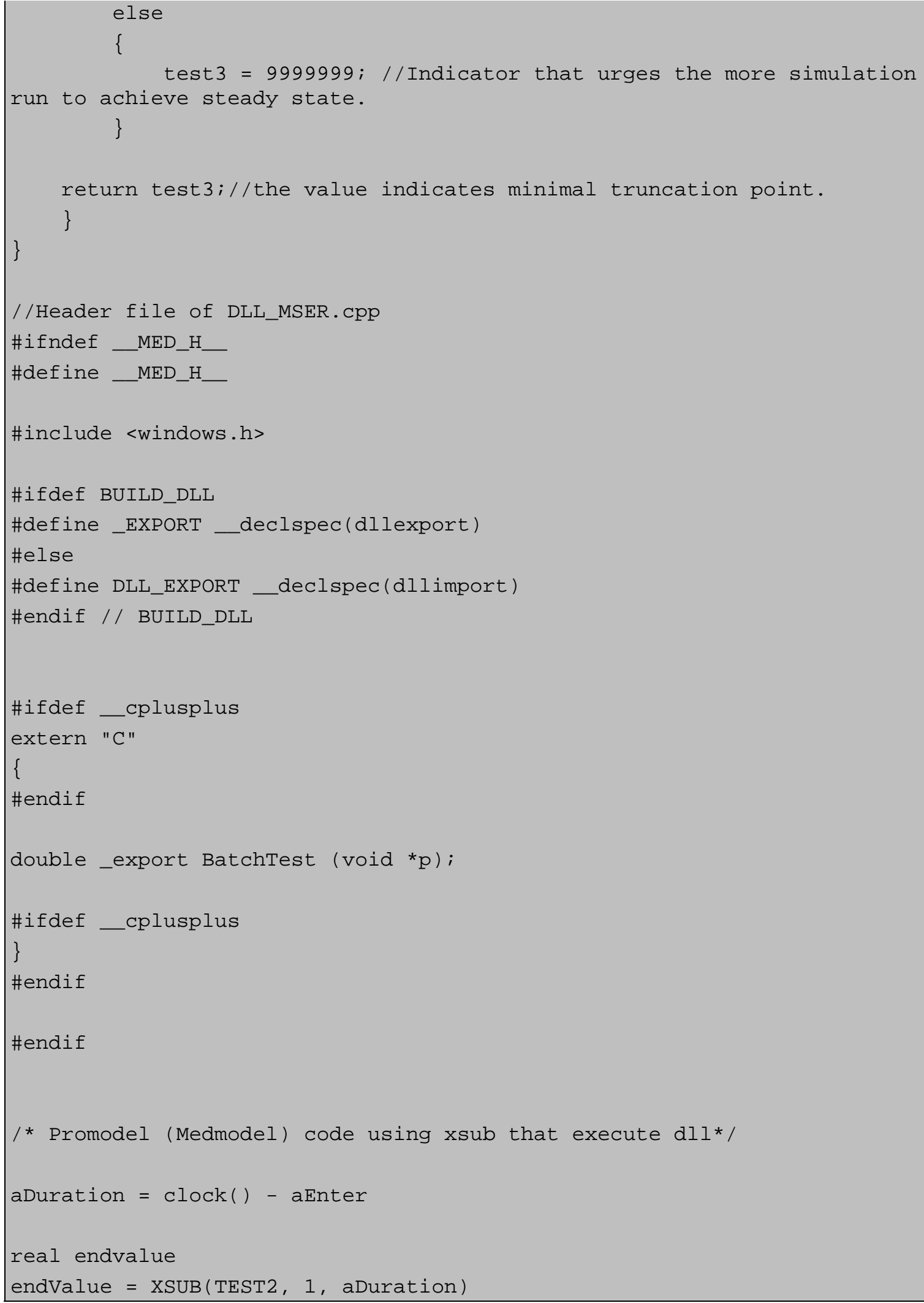




\section{Chapter 6. Implementation in Post Analysis Codes}

The MSER Laboratory currently includes five post-analysis codes written in R, SAS, Matlab, VBA, and $\mathrm{C} / \mathrm{C}++$. In this Chapter, we demonstrate the application of each of these codes on a simple example. The corresponding outputs also are provided.

Each code reads data from a file (either Simple2.csv or simple2.txt). These data are the output of one replication of a normal white noise process with superimposed deterministic bias initialized at declining from 15 to 0 with a slope of -0.1 . The batch size is set at $b=5$ and the run length is $n=10,000$ observations. The results from all five applications are identical. The sample mean without truncation is 0.106268 , the MSERoptimal truncated mean is -0.005395563 , and the MSER truncation point is $d^{*}=30$.

\subsection{R Source Code}

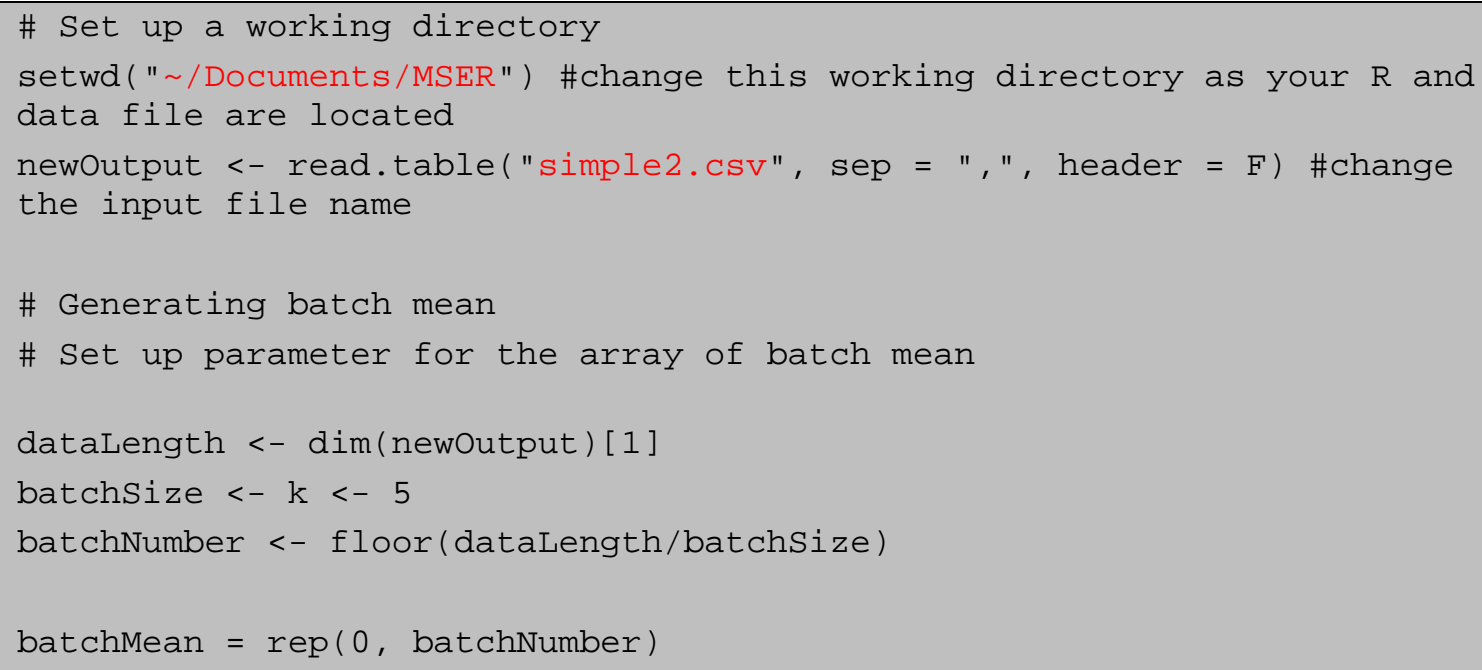




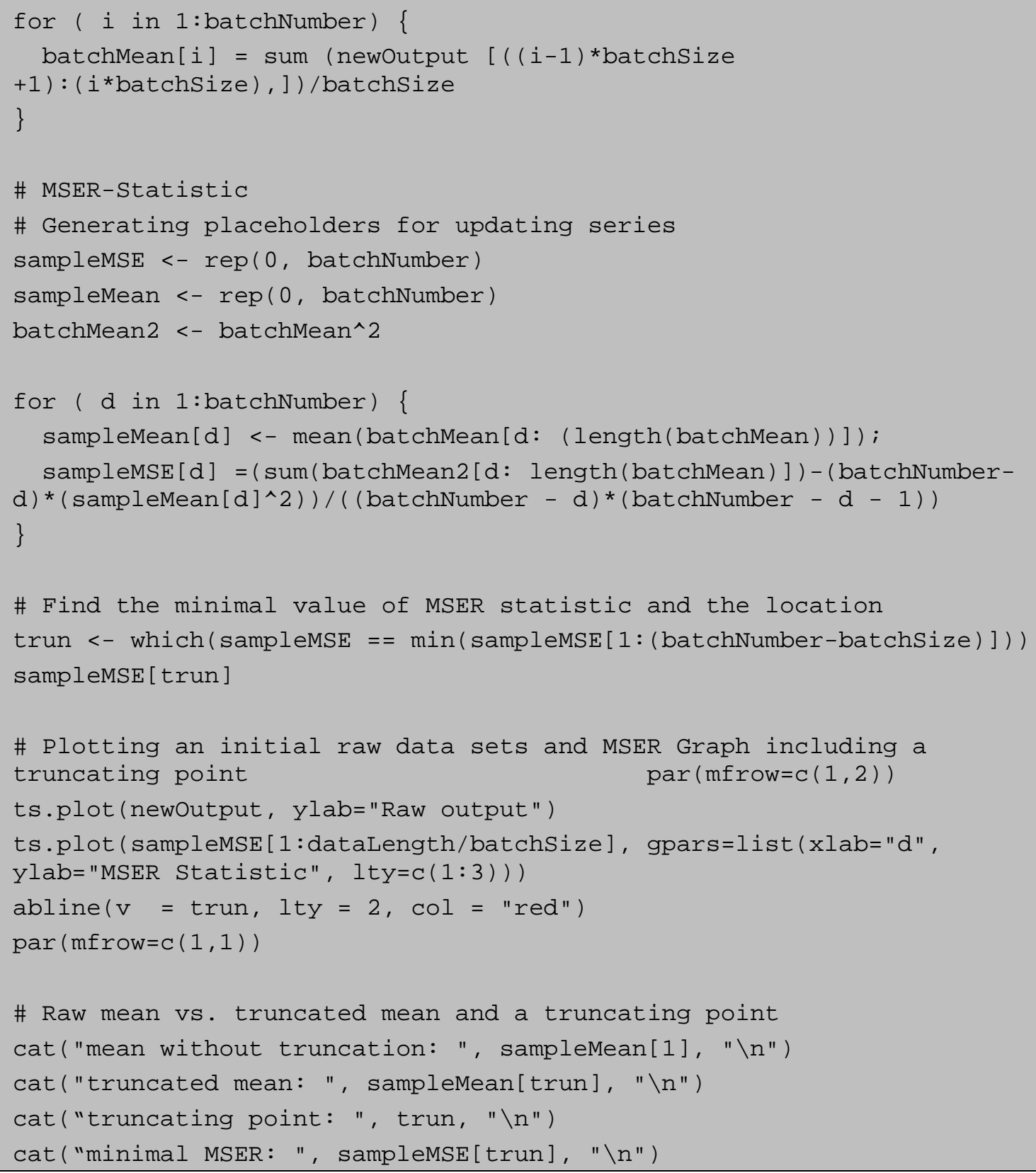




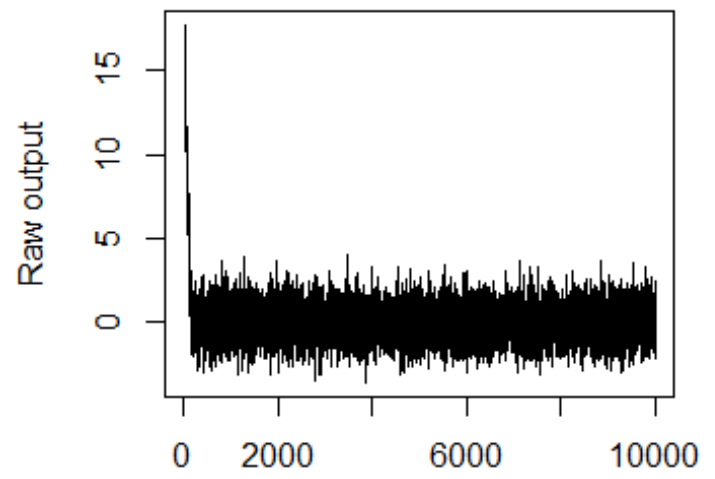

Time

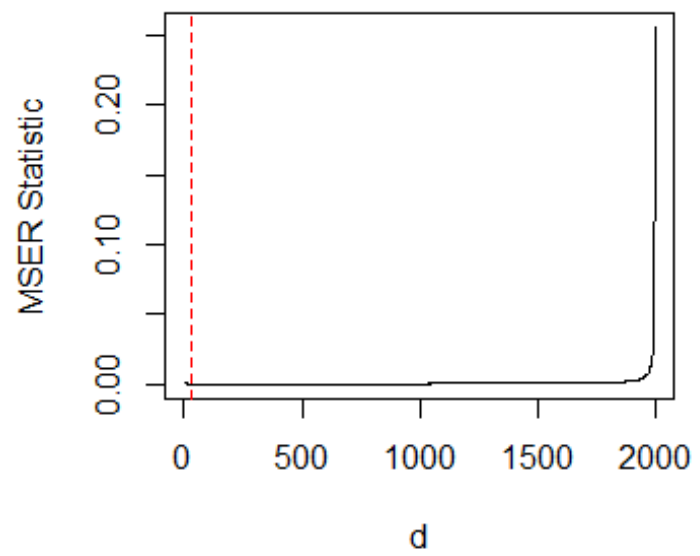

Figure 6.1 Output and MSER Plot in R

\subsection{The SAS Source Code}

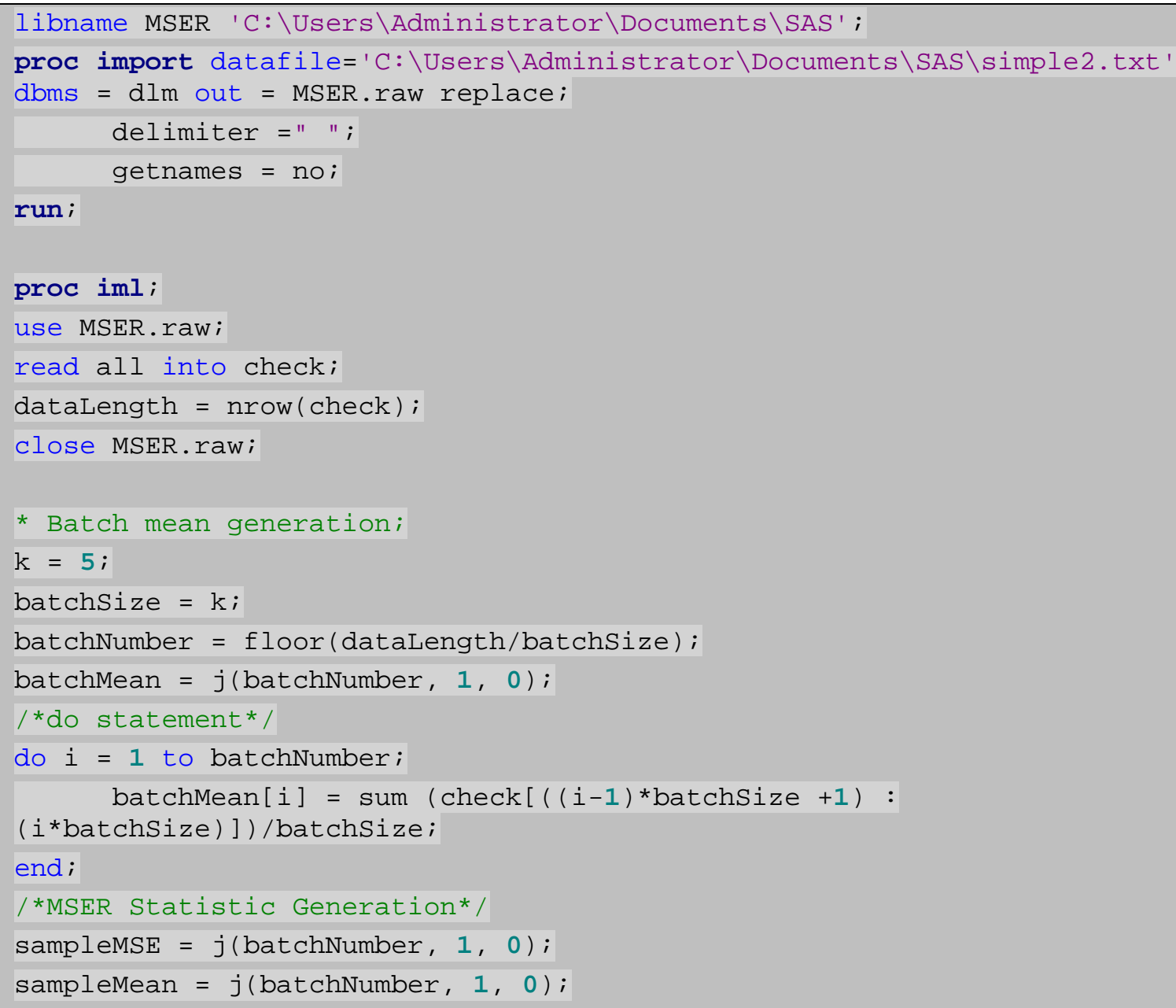



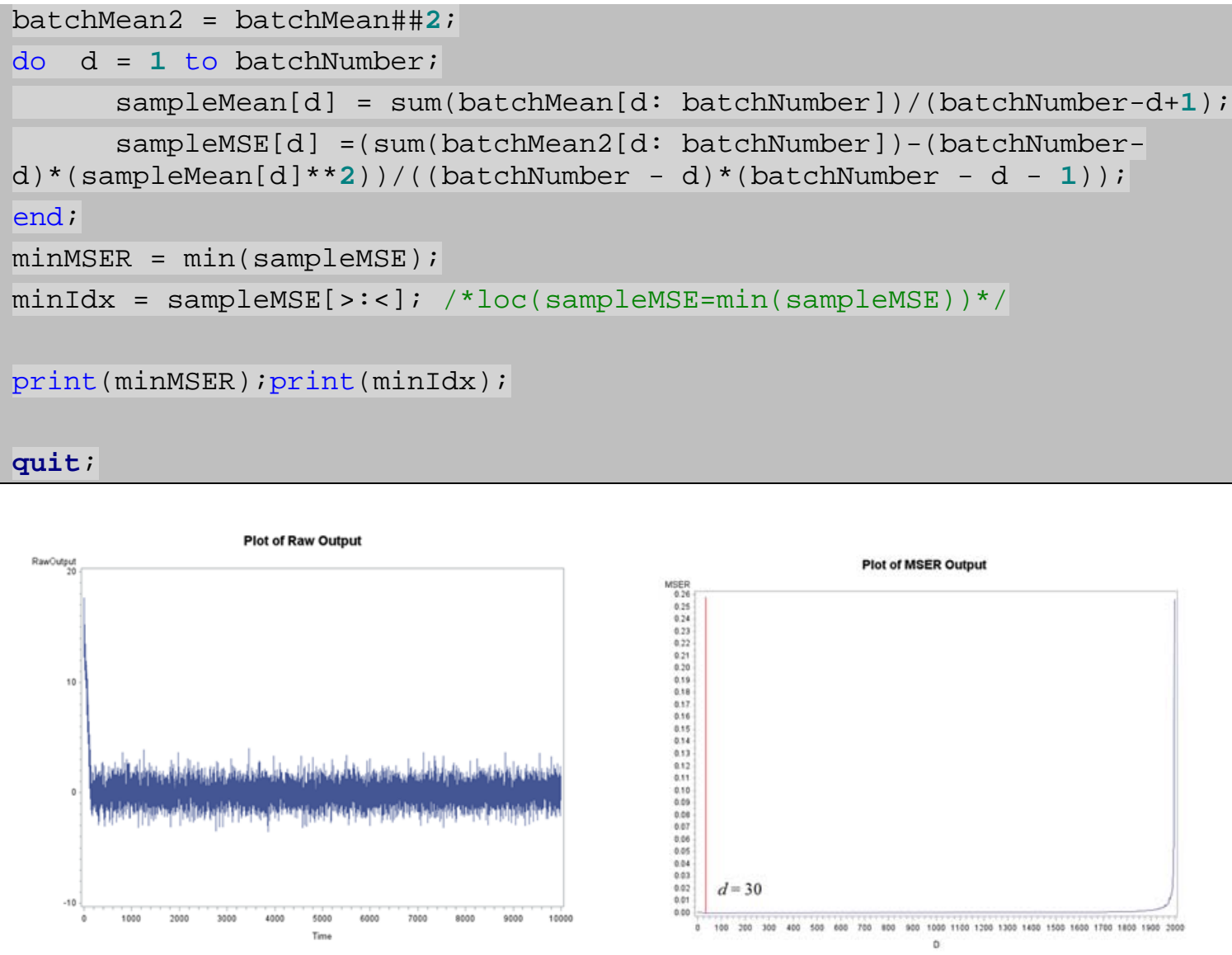

Figure 6.2 Output and MSER Plot in SAS

\subsection{Matlab Source Code}

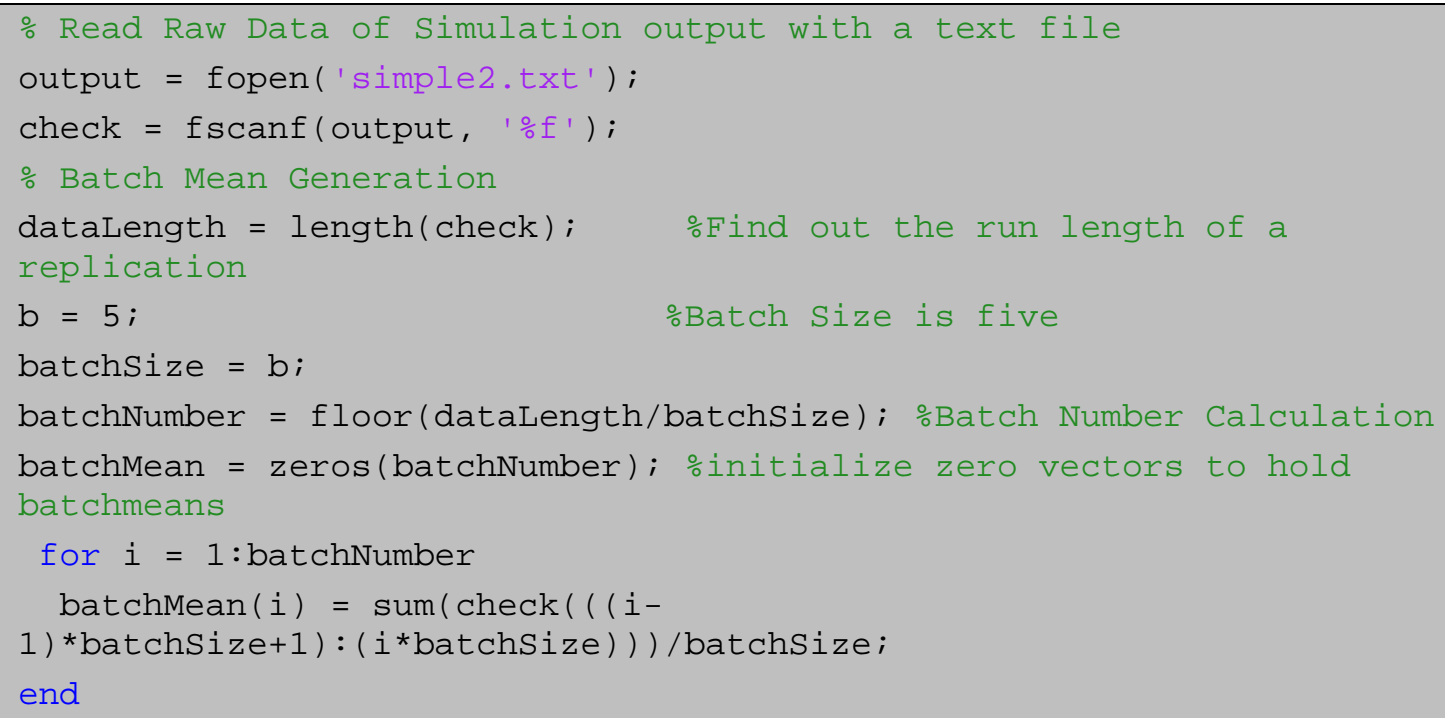



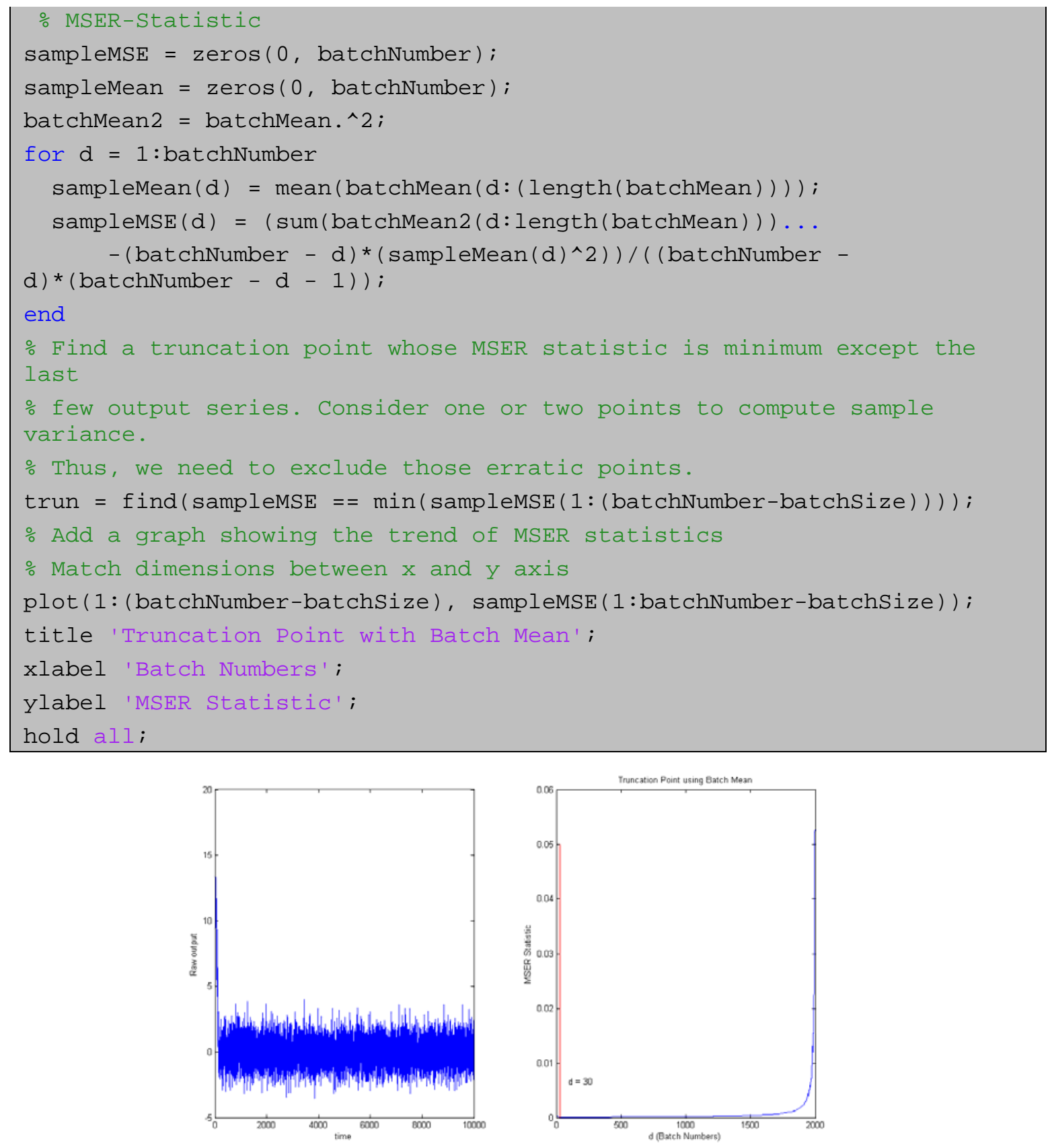

Figure 6.3 Output and MSER Plot in Matlab

\subsection{VBA Source Code}

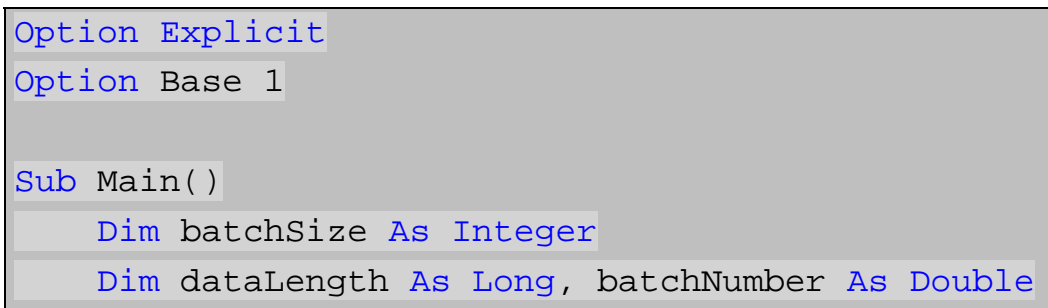




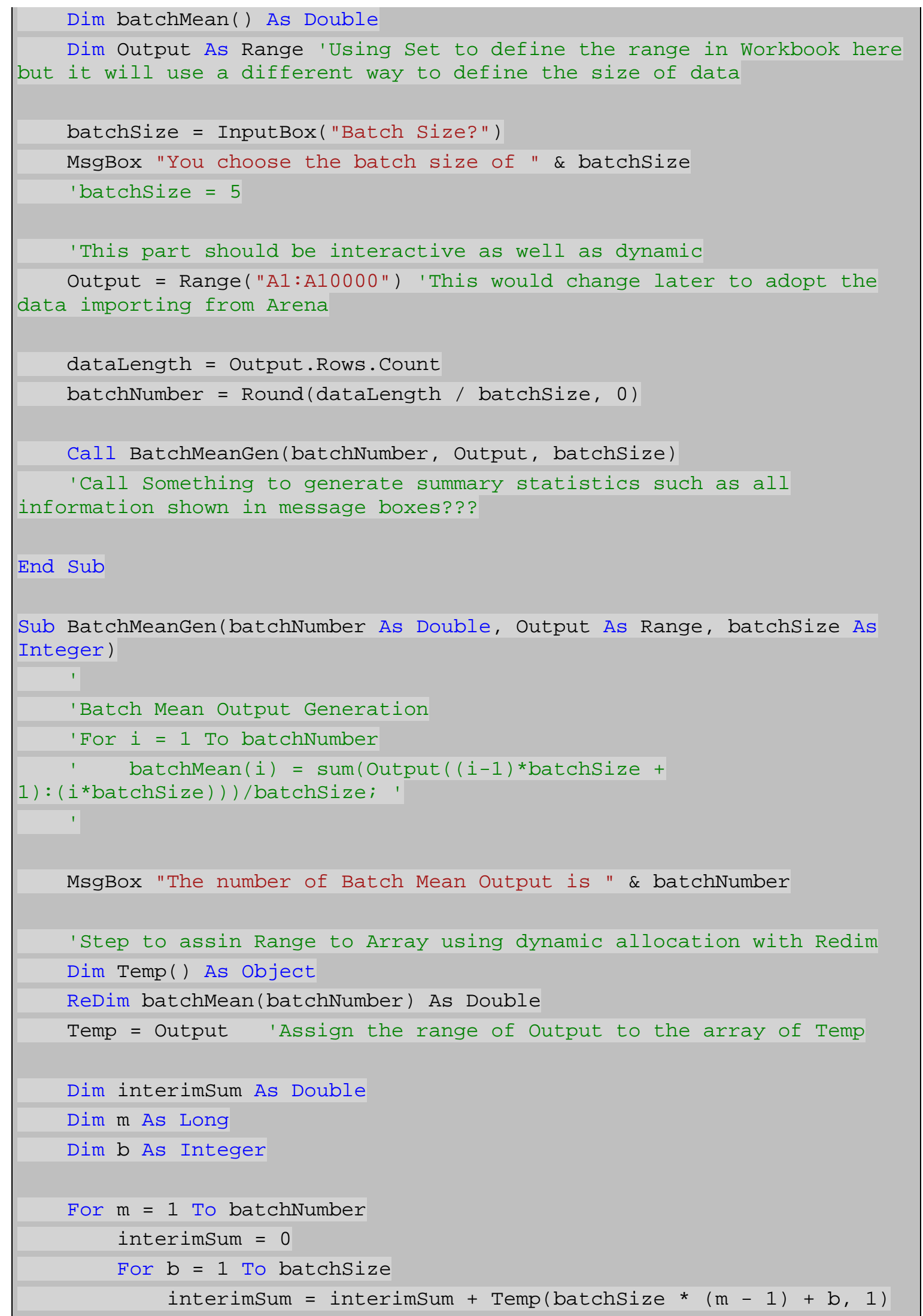




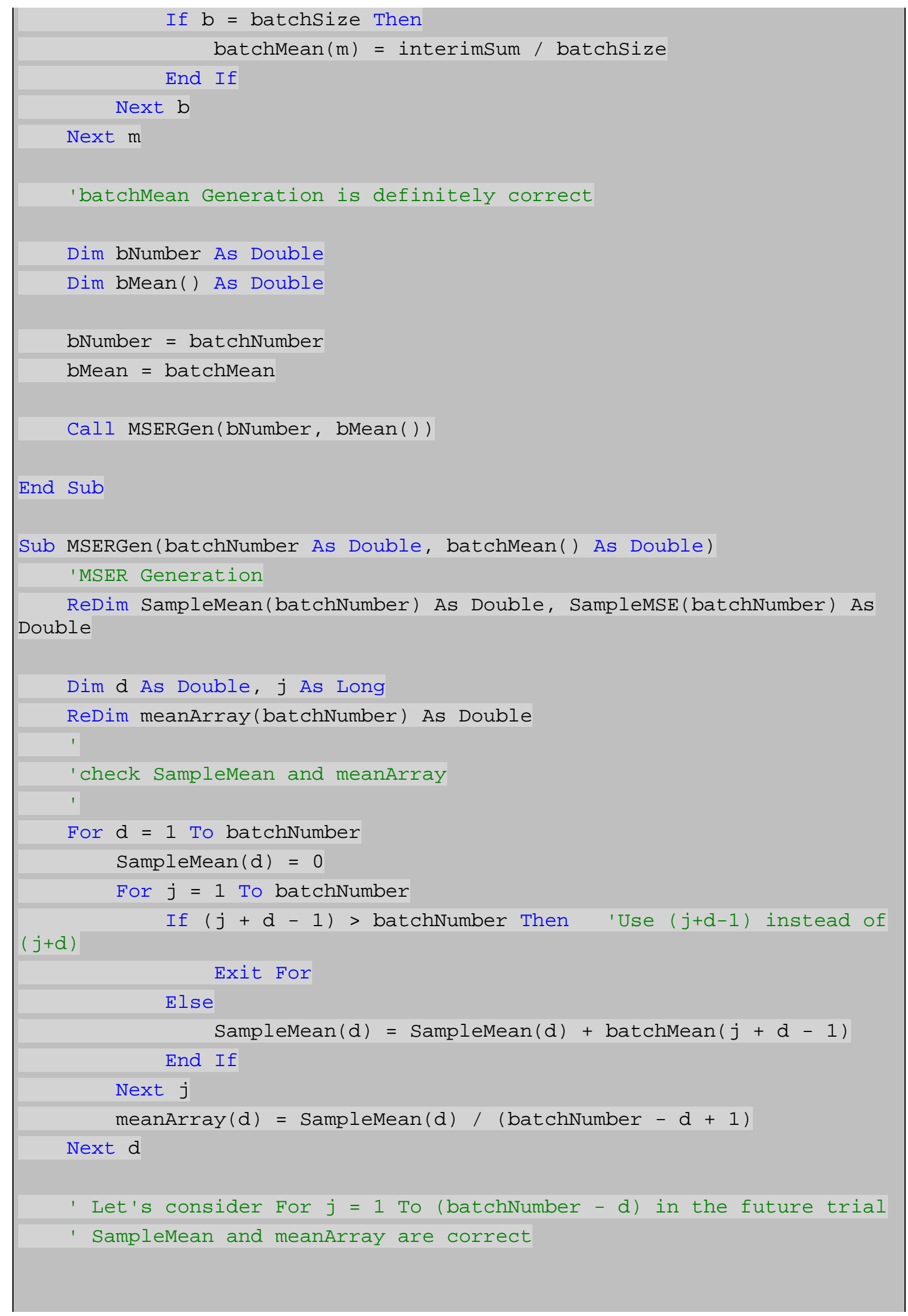




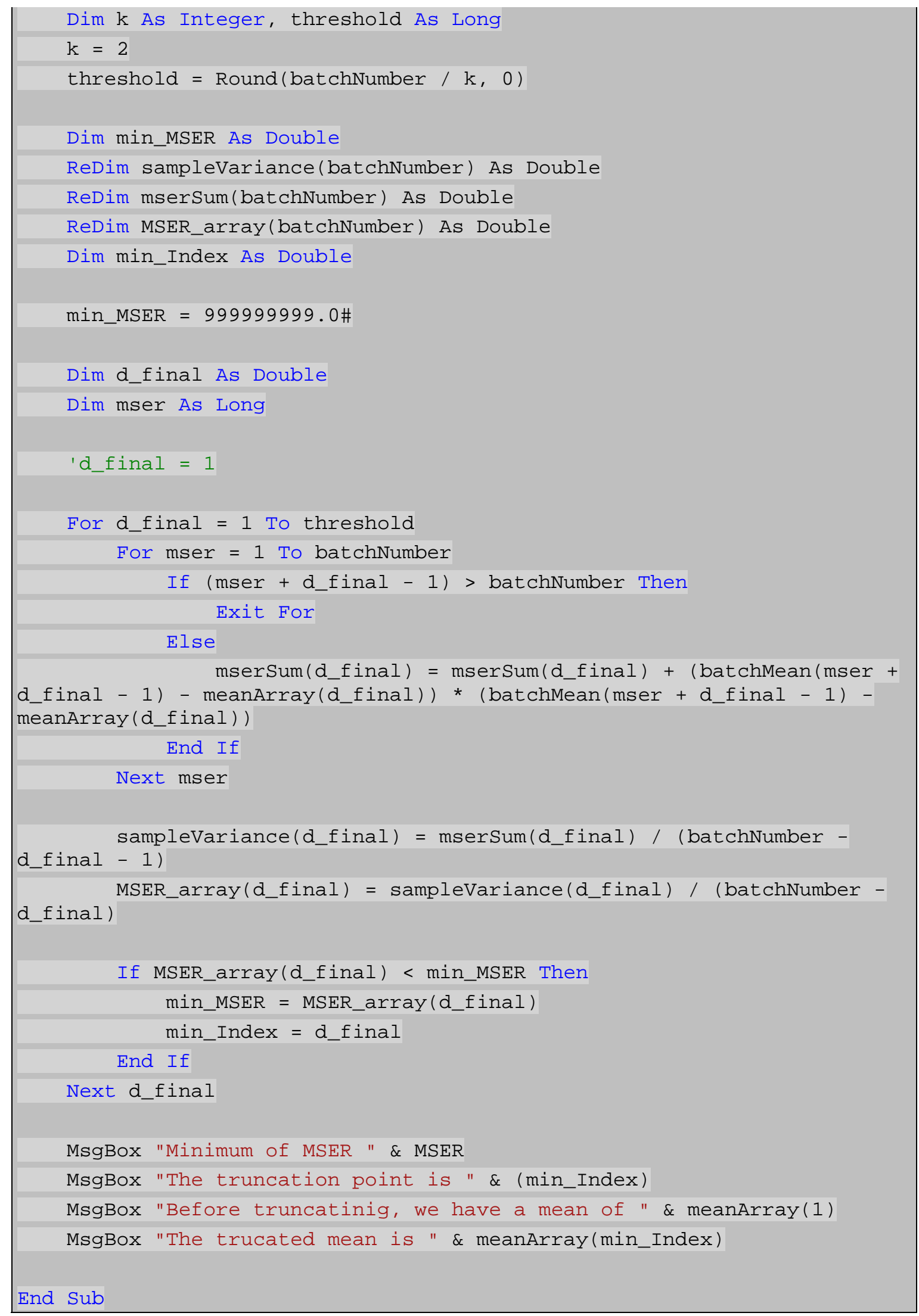



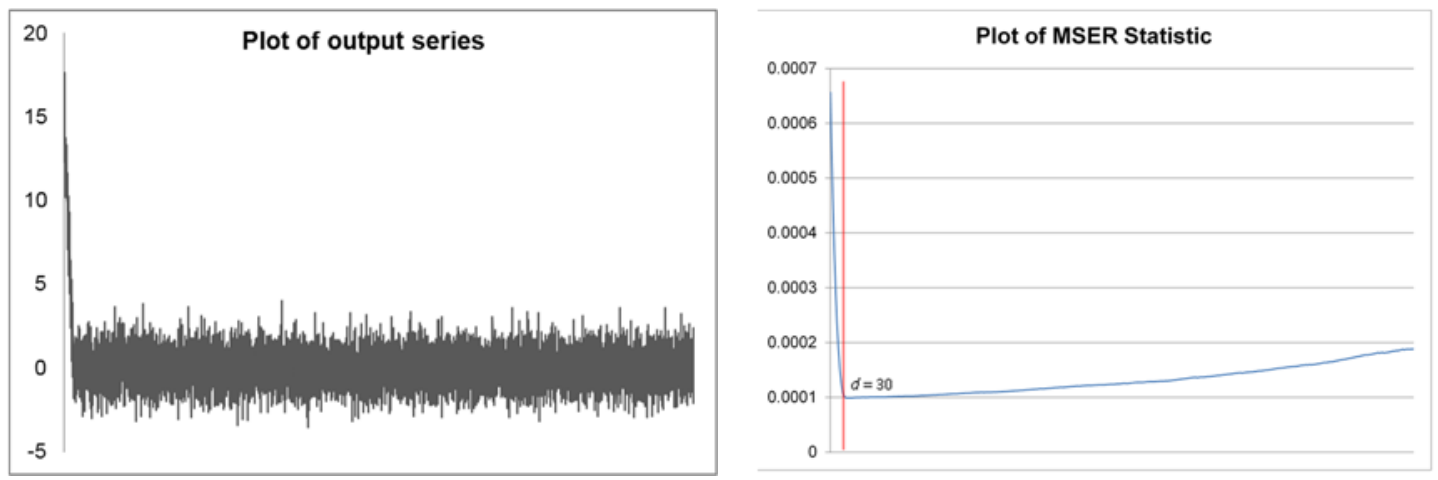

Figure 6.4 Output and MSER Plot in Excel VBA

\subsection{Source Code}

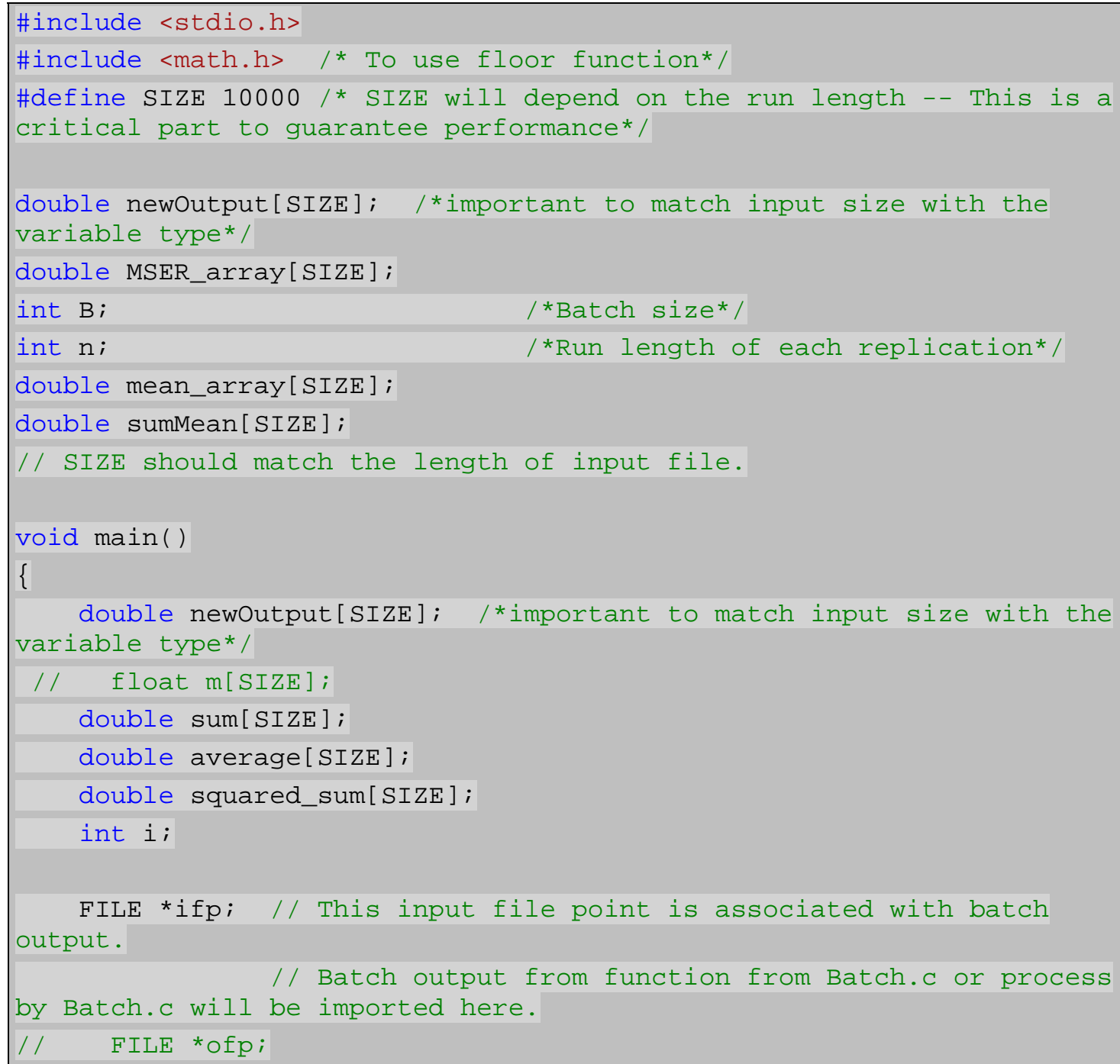




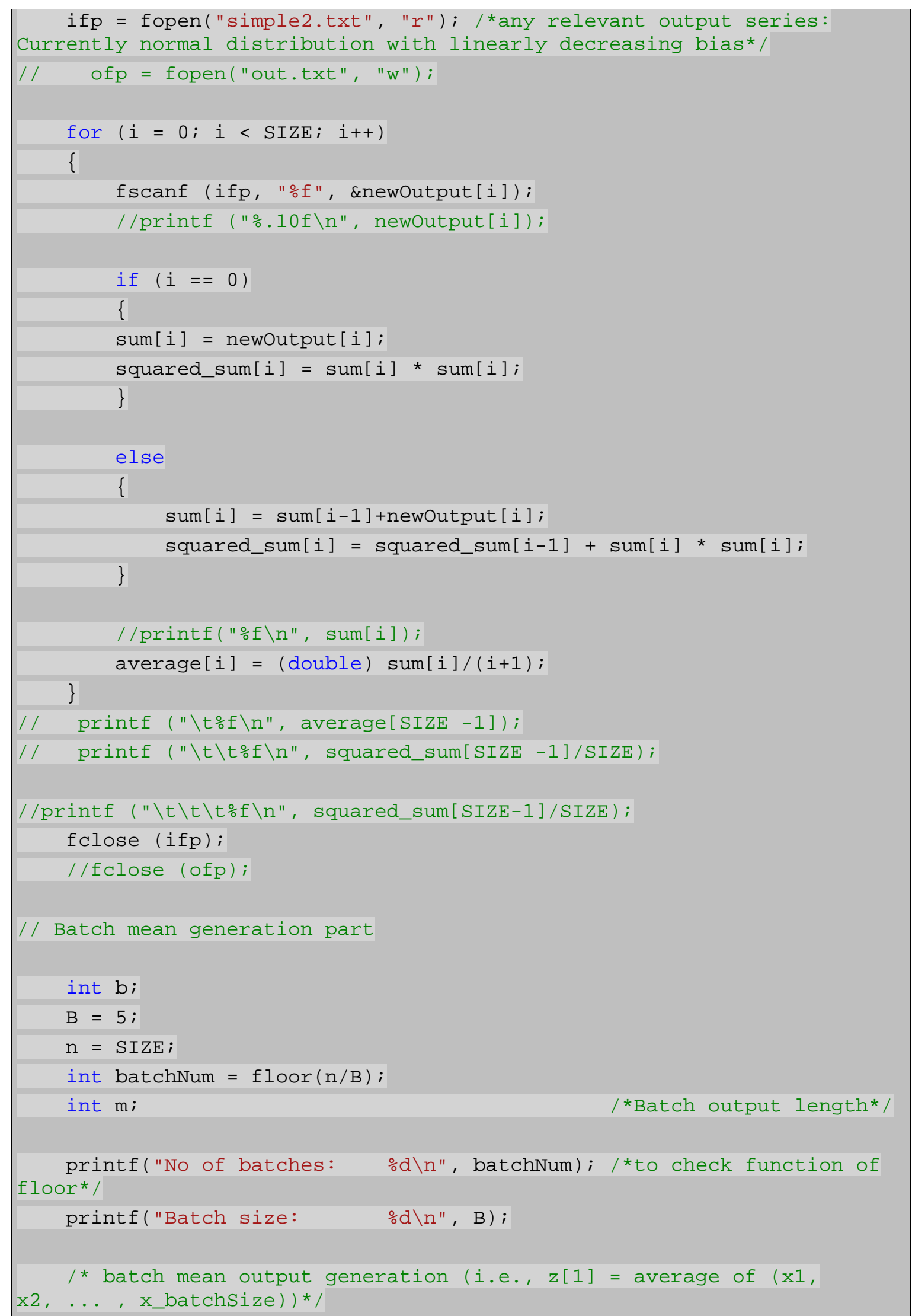




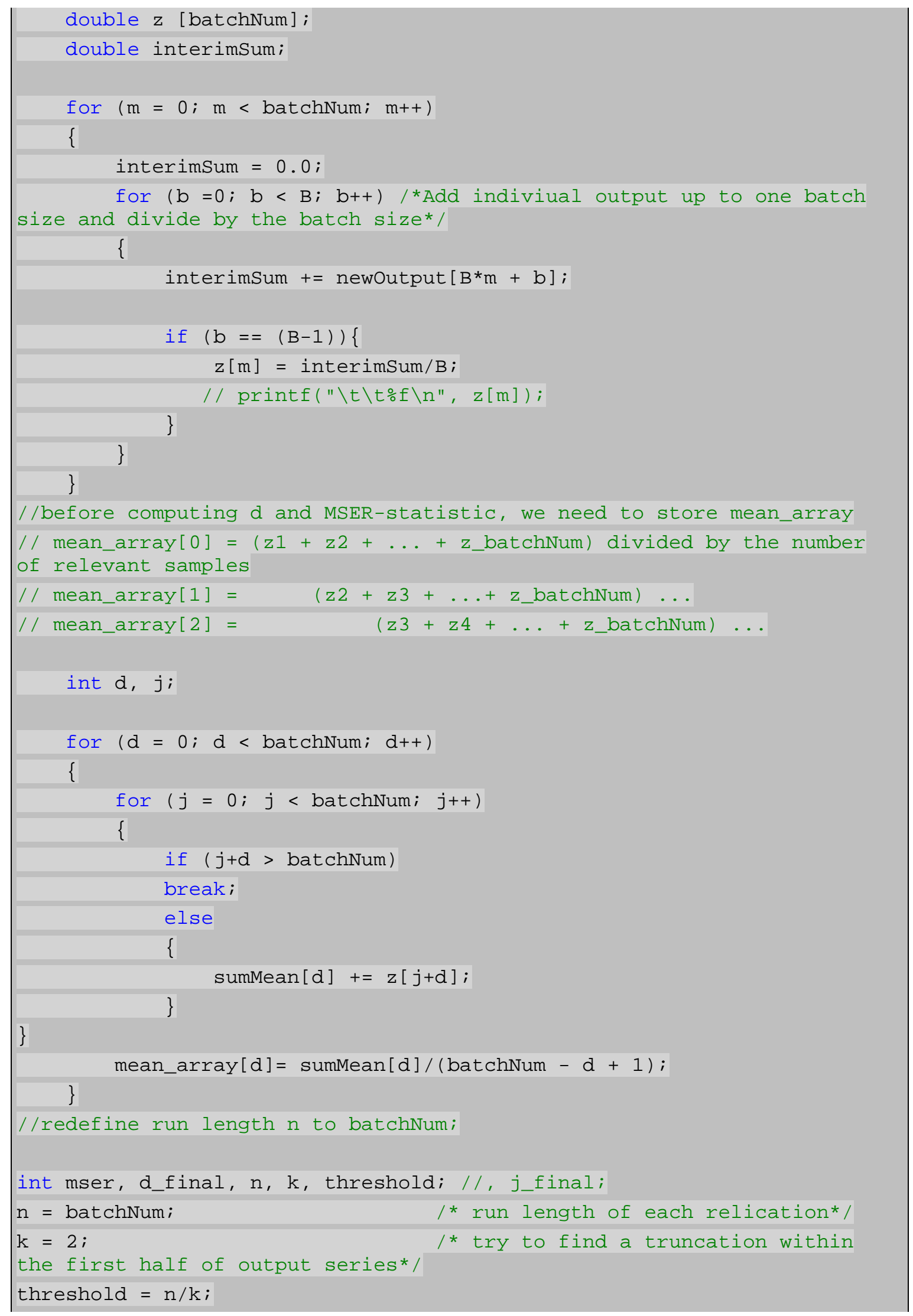




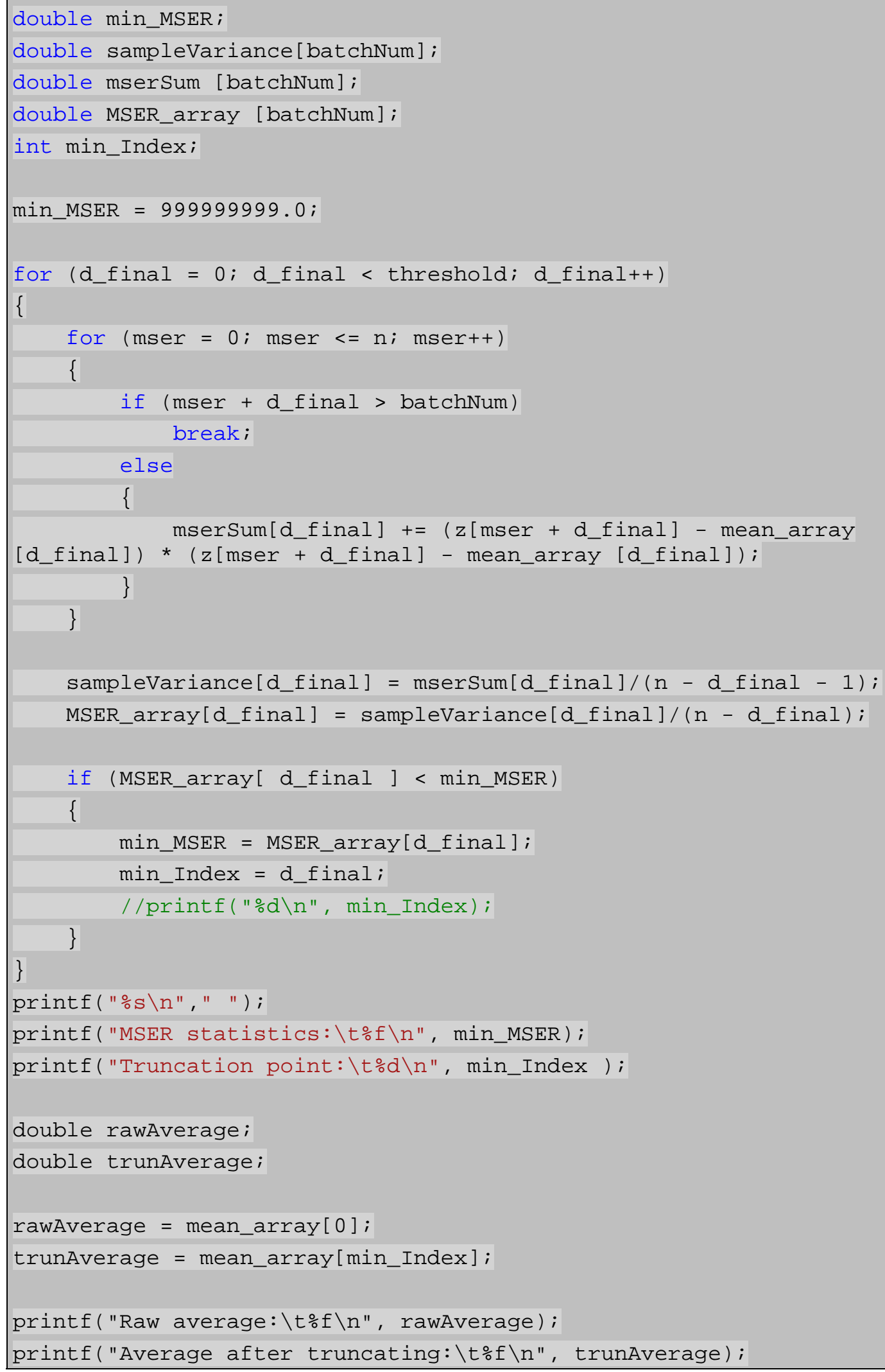




\section{6. $\mathrm{C}++$ Source Code}

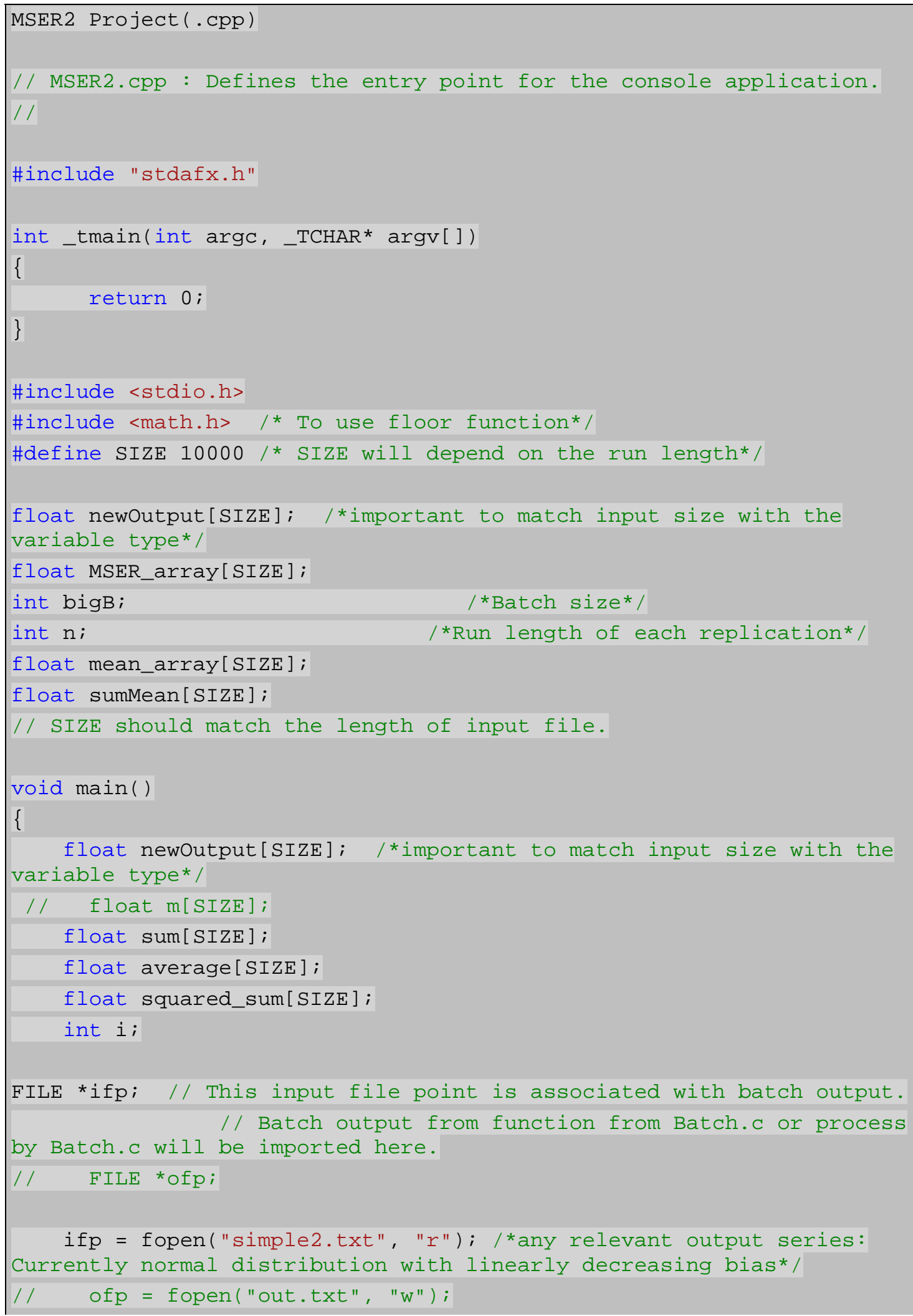




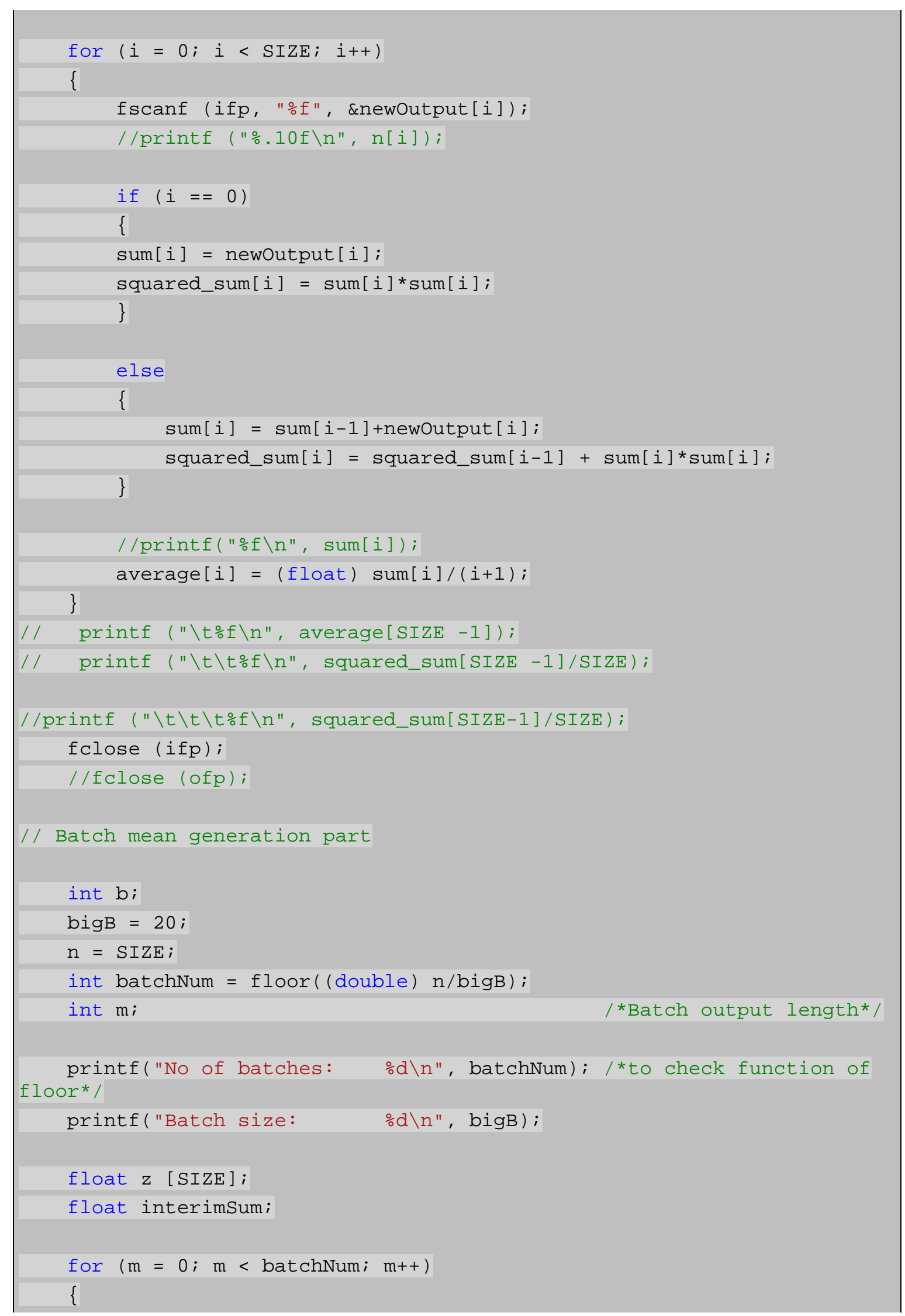




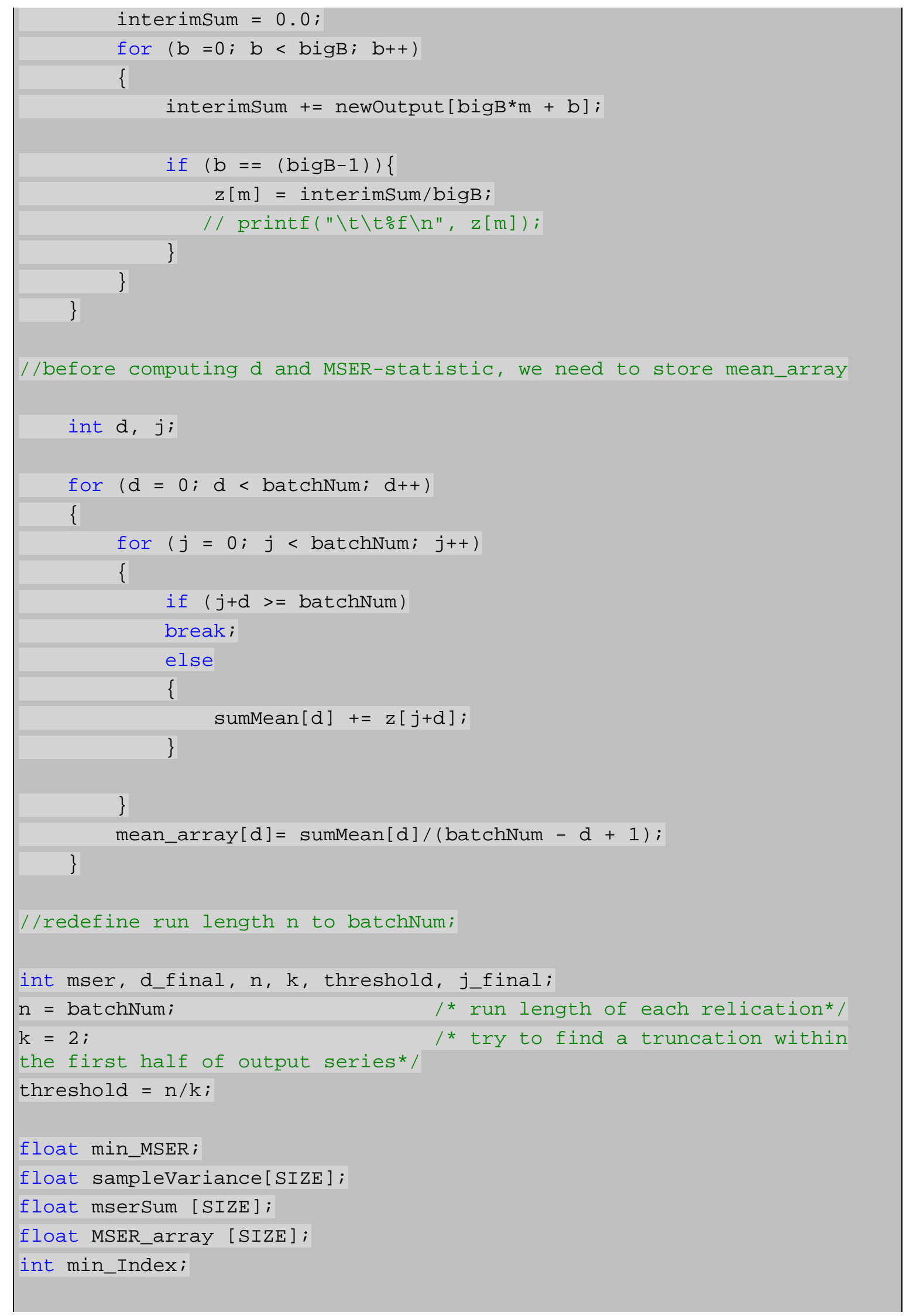




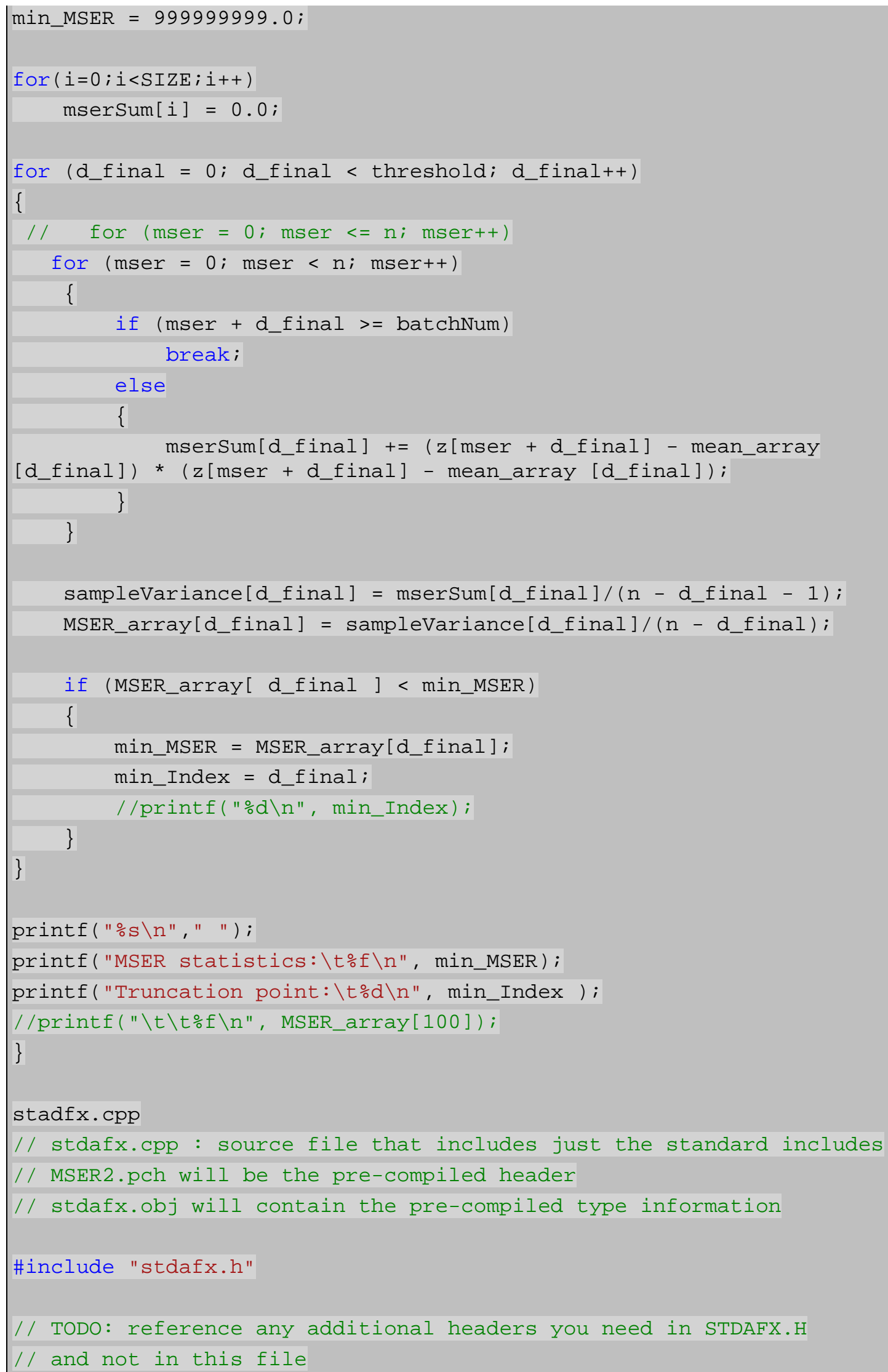




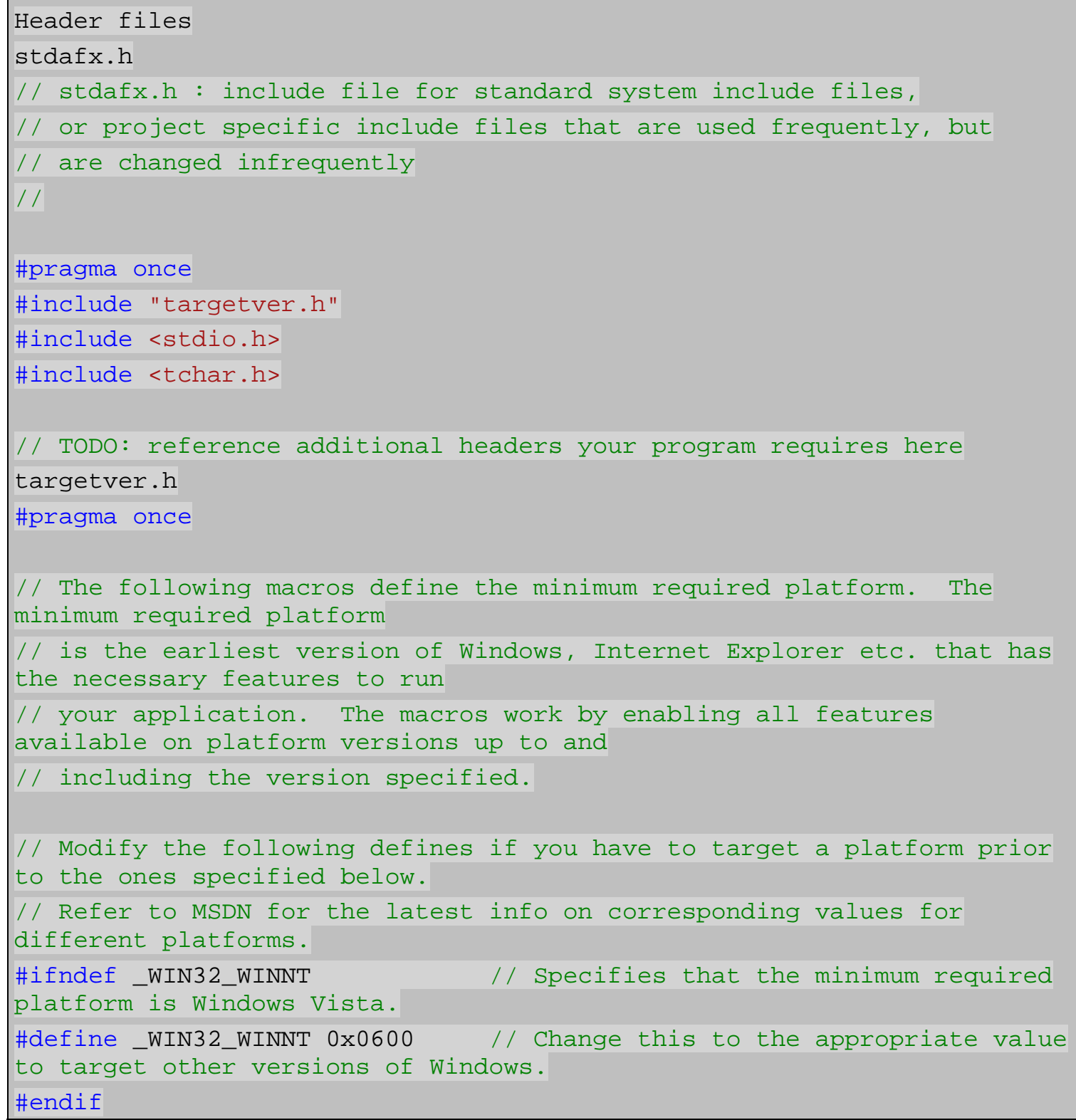




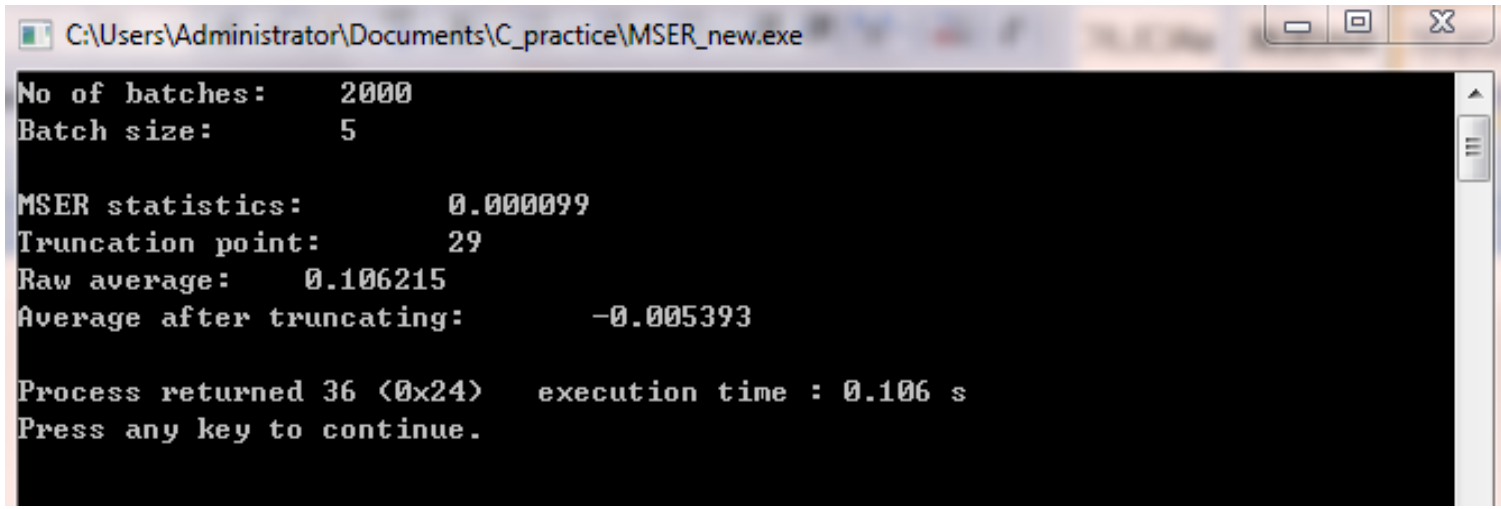

Figure 6.5 Output Results in Console by $\mathrm{C} / \mathrm{C}++$ 


\section{Chapter 7. Parameterization Issues, Analyses, and Results}

While MSER is widely accepted as the most robust approach to initializing steady-state simulations, there remain a number of open questions regarding its application. These include:

- the choice of simulation run length $n$,

- the choice of batch size $b$, and

- the maximum acceptable optimal truncation point $d_{\max }$ on the range of a given run length $\left[0 \leq d_{\max } \leq n\right]$

- the incorporation of the overlapping batch means.

One purpose of this research is to provide insight on the relationships among these parameters and guidance regarding their selection. To this end, we explore these relationships empirically on a selection of test problems using a replication/deletion analysis framework. We estimate the sampling distributions of the truncated means and corresponding truncation points, test for correlation, and compare response surfaces for varying batch sizes and run lengths. Before proceeding to the test problems, however, we consider a fundamental and perhaps unresolvable difficulty inherent in choosing an adequate run length (see White and Hwang, 2015).

\subsection{Choosing the Run Length of a Nonterminating Simulation}

While intuition may derive from the system being simulated and/or the purpose of the simulation study, determining an appropriate run length for a nonterminating simulation 
typically is a process of trial and error. Framed as an optimization problem in Chapter 2, the global objective is to discover the number of observations needed to achieve both the accuracy and precision desired in the estimated steady-state simulation outputs with the minimum computing effort. For output series that are potentially nonstationary or cyclical, run lengths must first and foremost be long enough to convince one that this is or is not the case; for output series that are slow to converge in distribution, practical constraints on time and computing budgets may necessitate settling for shorter run lengths and confidence intervals wider than desired.

We distinguish between the two typical output analysis frameworks. If batch-means is adopted for output analysis then the run length should be sufficient to provide a sample of steady-state observations (after truncation sufficient to mediate initialization bias) large enough to form nearly uncorrelated batch means yielding a desired level of confidence in the grand mean. If replication/deletion is adopted, then each of the replication run lengths should be adequate to guarantee that MSER can determine an appropriate truncation point. In this case, the accuracy and precision of the estimate is a function of the number of replications run. The interested reader should see Hoad, et al. (2007) for a review of the literature and an empirical comparison of alternative procedures for determining the number of replications required.

The replication/deletion framework provides the insights we seek regarding MSER parameterization. MSER works by successively considering the leading observation $x_{d}$ in the output series $\left\{x_{d}, \ldots, x_{n}\right\}$, i.e., the sequence remaining after the initial $d$-1 observations have been truncated. This observation is a candidate for deletion if the MSE in the estimate of the steady-state mean decreases for the reserved sequence $\left\{x_{d+1}, \ldots, x_{n}\right\}$. For MSER to 
succeed in determining an optimal truncation point for any single replication, however, the algorithm has to "see" enough of the steady-state response in the reserved sequence in order to make a correct determination. We note that, symptomatically, as the sample size $n$ - $d$ becomes small with continued truncation, the MSER statistic can behave erratically (Rossetti, Delaney, and White, 1995; Spratt, 1998; and Hoad et al., 2008). In other words, there is some shortest sequence $\left\{x_{\operatorname{dmax}}, \ldots, x_{n}\right\}$ that is "sufficiently large and representative" of a sample drawn from the steady-state distribution of $X_{t}$ for use in determining a truncation point.

If MSER determines that $d^{*}>d_{\max }$, then by construction the algorithm should return a message that an optimal truncation point cannot be determined for the given output sequence. In such cases the MSER statistic decreases over entire range $\left[0, d_{\text {max }}\right]$, indicating that the output sequence increases (in trend) or decreases over this same range. This will be the case for one of two reasons. Either (1) the output is inherently unstable (such as a queue with traffic intensity $\varrho \geq 1$ ) and MSER will not converge irrespective of run length, or (2) the process is stable, but the run length $n$ is insufficient to achieve a detectable steady state. Without addition computing, it is impossible to tell which is the case based on the output alone.

To illustrate the inherent problem, consider output sequences for two different systems given in Figure 7.1. First, consider run lengths of $n \leq 500$. The MSER statistic will have a minimum value in the neighborhood of $n$ for both Output A (Red) and Output B (Blue). MSER therefore will fail to return a truncation point for either output. 


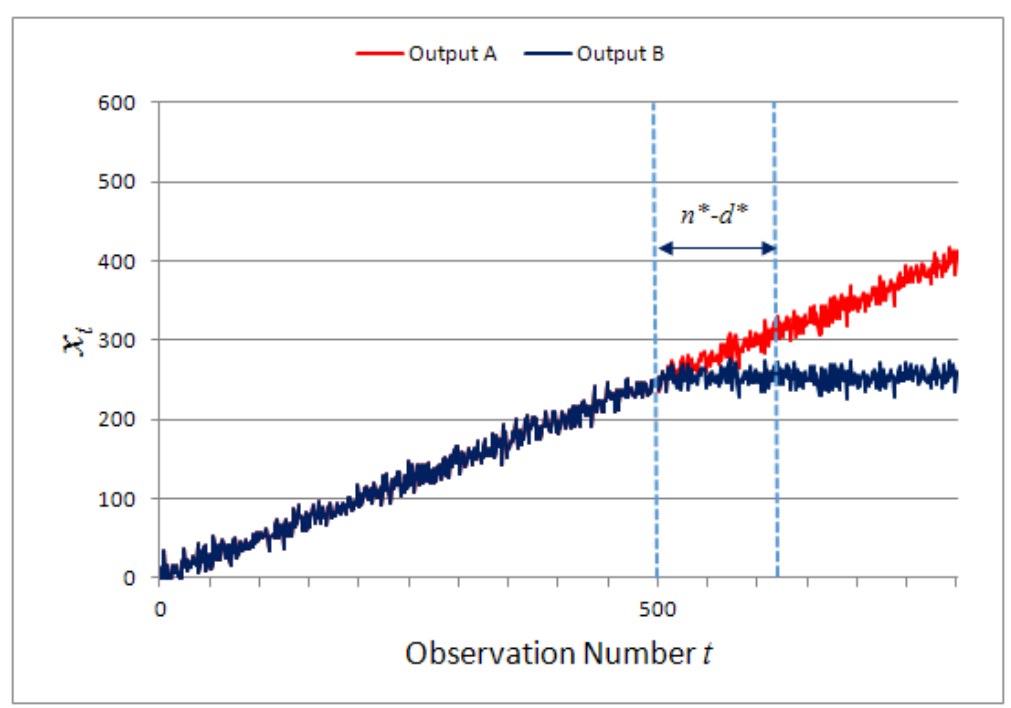

Figure 7.1 Hypothetical simulation output sequences illustrating one potential consequence of an inadequate run length

Second, consider run lengths of $n \geq 500$. MSER will still fail to return a truncation point for Output A. However, if $n$ is sufficiently large, MSER will return optimal truncation point in the neighborhood of $d^{*}=500$ for Output B. It is unlikely that MSER will find an optimal truncation for values all values of $n \geq 500$ and the question is, "What is the minimum run length $n^{*}$ such that MSER finds a correct truncation point for Output B?" This almost certainly will depend on the unique properties of the specific output sequence under consideration, most especially the degree of sequential correlation.

Now consider output sequences for two different systems given in Figure 7.2. These are possible extensions of the outputs in Figure 7.1. Obviously, MSER will yield the same results for run lengths $n \leq 800$. If $n$ is sufficiently large, however, MSER will conclude (1) that Output A (Red) has stabilized, and will return optimal truncation point in the neighborhood of $d^{*}=800$; and (2) Output B (Blue) is unstable and fail to return a truncation point for this output. Again, the question of how large is sufficient to draw either conclusion remains unclear. 


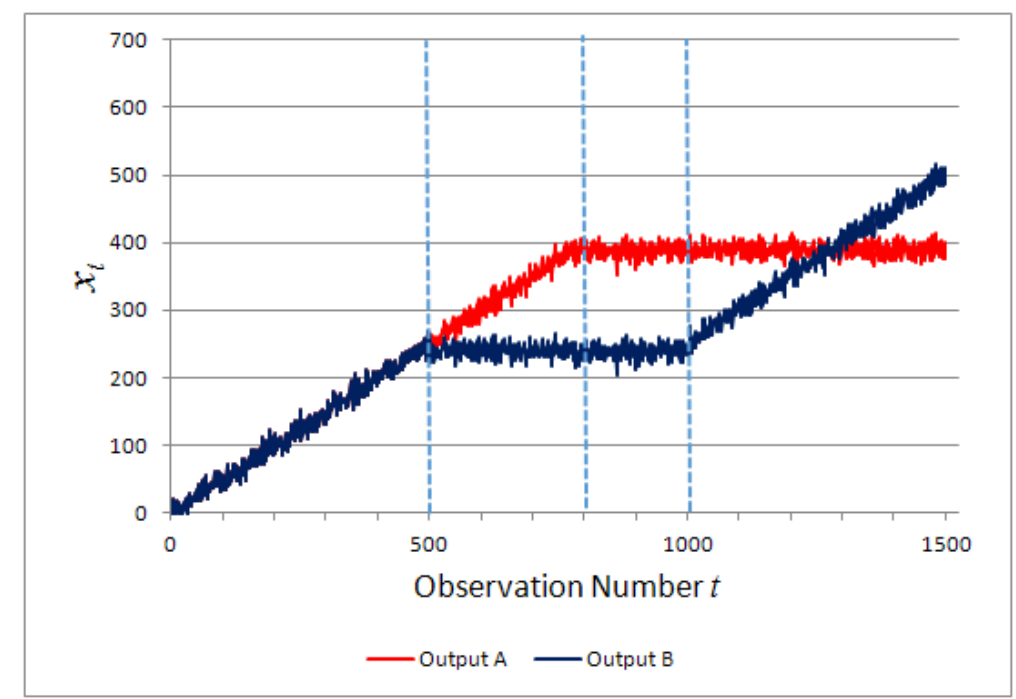

Figure 7.2 Hypothetical extensions of the simulation output sequences in Figure 7.1 illustrating further potential consequences of an inadequate run length

From these examples we see that MSER can potentially change its determinations regarding the location and the even the existence of a suitable truncation point depending on the run length chosen. While in our experience Output B in the second example is a pathological case, the conclusion remains that the performance of MSER depends on choosing a sufficient run length. And without knowledge beyond the output sequences alone, there are no guarantees that this is or is not the case.

\subsection{Test Models and Results}

We performed empirical tests with differing batch sizes to determine if any discernable trends or correlations exist among mean estimates, truncation points, batch sizes, and run lengths, using three different forms of output. These test outputs included (1) the response of a uniform white-noise process in steady-state with a superimposed linearly-decreasing deterministic transient, (2) the delay times in an M/M/1 queue, and (3) the response of an $\operatorname{EAR}(1)$ process. 


\subsubsection{Model 1: Uniform Distribution with Superimposed Deterministic Bias}

\subsubsection{Model Description}

The first test model is

$$
X_{t}=A_{t}+\varepsilon_{t}
$$

for $t=0,1, \ldots, n$, where

$$
A_{t}= \begin{cases}0.1(150-t) & , t=0,1,2, \ldots, 150 \\ 0 & , t>150\end{cases}
$$

and

$$
\varepsilon \tau \sim \operatorname{UNIF}(0,1)
$$

This is a special case of the family of models variously explored by Cash et al. (1992), Spratt (1998), White et al. (2000), and Hoad et al. (2008). As illustrated in Figure 7.3, the model consists of two parts: (1) At, a deterministic, additive, initial transient that declines linearly from 15 at 0 time units to 0 at 150 time units and (2) $\varepsilon$, a uniform white-noise process in steady-state, i.e., observations sampled randomly from a uniform distribution between zero and one. In steady state, $X_{l}=\varepsilon_{t}$ so that $E\left[X_{t}\right]=0.5$ and $\operatorname{VAR}\left[X_{t}\right]=1 / 12$.

We selected this model as the baseline for testing MSER performance because of its transparency - the transient expires at $t=150$ and steady state comprises observations that are positive recurrent on the continuous range $\varepsilon_{t} \in[0,1]$. These white-noise observations are uncorrelated by definition and therefore the mean transient (White and Robinson, 2010) settles relatively quickly after truncation. Additionally, the state-transient sequence $A_{t}$ includes transient (nonrecurrent) observations on the discrete ranges and $A_{t} \in[1.1,1.2$, $\ldots, 15]$ for $t<140$ and $A_{t} \in[0,0.1,0.2, \ldots, 1] \subset[0,1]$ for $t \geq 150$. Therefore, as apparent 
in Figure 7.3, we can easily detect near-optimal truncation point $d$ visually, somewhere on the range $d=t \in[140-150]$, depending on the values realized for the noise on this range for any particular output sequence. Because the transient observations are linearly decreasing (in trend), the probability that observations $x_{t} \leq 1$ for all $t>d$ increases (in trend) from 0 to 1 as $d$ increases on this range.

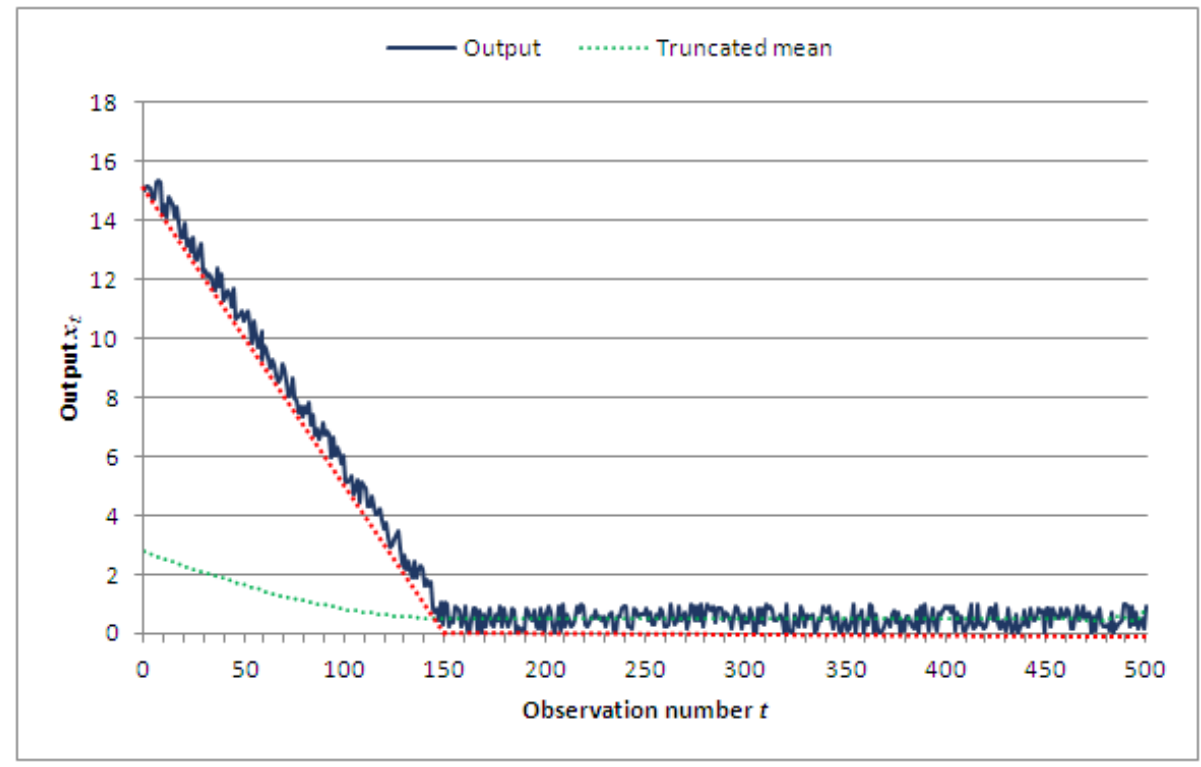

Figure 7.3 Output for a representative replication of the first model

It is important to note the MSER truncation point $d^{*}$ may differ from the "true" truncation point ( $d=150$ for this model). This is because MSER will retain observations from the transient sequence, or delete observations from the steady-state sequence, if (and only if) this improves the estimate in the sense of minimizing the MSE. Determining the "true" truncation point is used as a performance criterion in several studies (see, for example, Hoad et al. (2008) and Law (2015)), where failure to select the "true" truncation point is viewed as a potential shortcoming of MSER - i.e., as "consistent underestimation or overestimation of the true end of the initial. Clearly, this ignores the fact that MSER 
leads to superior estimates in the sense of MSE by not selecting the "true" point (White and Hwang, 2015).

\subsubsection{Results: Batch size effects for long runs}

To explore the combined effect of batching and run length on MSER performance, we ran 1,000 independent replications of Model 1 with batch sizes $b=1,5$, and 10. Initially, we chose a run length of $n=10,000$ observations, noting that this run length should be several orders of magnitude longer than the state-transient sequence regardless of the batch size.

Table 7.1 and Figure 7.4 confirm the effectiveness of MSER truncation for the first model in terms of both the accuracy and precision of the estimated steady-state mean. While the confidence intervals for batch sizes of 5 and 10 do not quite cover the true mean, all of the estimates are accurate to three significant figures. Note, also, that the small errors in the estimates are all negative, while the biasing observations are greater than the steadystate mean. From this we conclude that for this instance

(1) all batch sizes remove all of the transient observations, and

(2) estimation errors are an artifact of sampling after the biasing effect of the initial transient has been removed.

As shown in Figure 7.5, the sampling distributions of the mean are nearly normal, as predicted by the Central Limit Theorem, and nearly identical for all batch sizes. From this we conclude that for this instance

(3) modest batching has no significant effect on the quality of estimates. 
Table 7.1 95\% confidence intervals for the mean and variance for the truncated mean output as a function of batch size for batch sizes $b=1,5$, and 10 for run length $n=10,000$

\begin{tabular}{|c|c|c|c|}
\hline Truncated Mean & Batch Size 1 & Batch Size 5 & Batch Size 10 \\
\hline Sample Mean & 0.499825 & 0.499789 & 0.499777 \\
\hline Upper limit & 0.500000 & 0.499965 & 0.499955 \\
\hline Lower limit & 0.499649 & 0.499614 & 0.499602 \\
\hline Sample Std Dev & 0.002850 & 0.002851 & 0.002829 \\
\hline Upper limit & 0.002960 & 0.002961 & 0.002959 \\
\hline Lower limit & 0.002711 & 0.002712 & 0.002711 \\
\hline
\end{tabular}

Table 7.2 shows that the mean number of observations truncated (as distinct from the number of batches truncated) is an increasing function of batch size. For batch size of $b=1$, the mean truncation is between $t=145$ and $t=150$, as anticipated. For batch sizes of $b=5$ and 10 , the means are modestly larger. These differences are statistically significant at the $95 \%$ confidence level with greater truncation for larger batches on average.

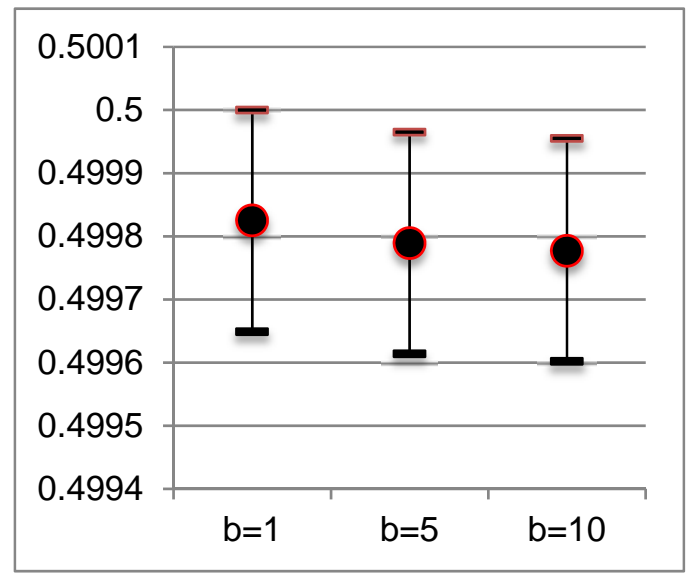

(a)

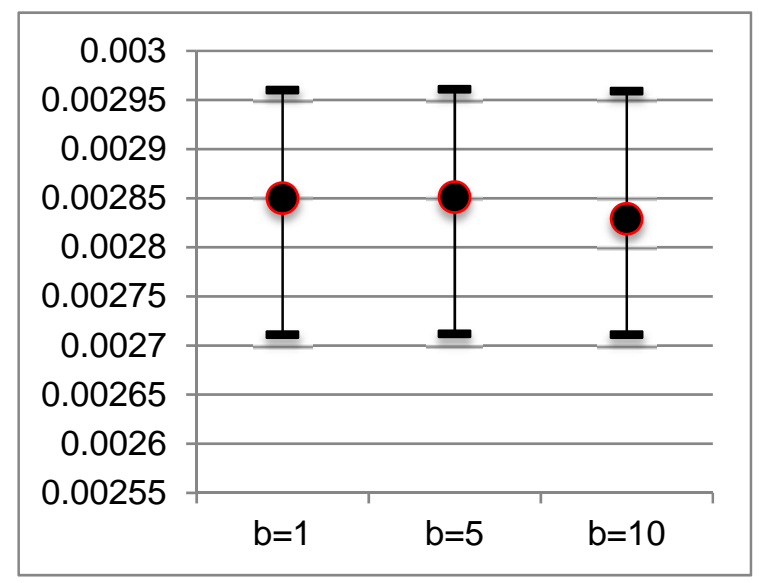

(b)

Figure $7.495 \%$ confidence intervals for (a) the truncated means for run lengths $n=10000$ and batch sizes $b=1,5,10$ and (b) the sample standard deviation in the estimated means for run lengths $n=10000$ and batch sizes $b=1,5,10$ 

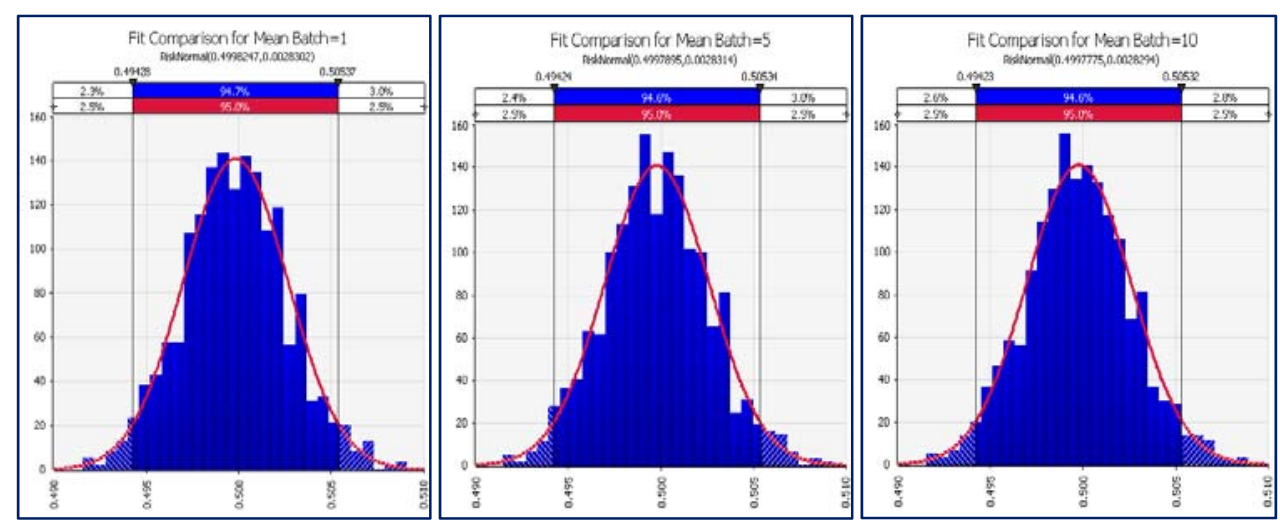

Figure 7.5 Fits to the steady-state sampling distributions of the mean for 1000 replications for run length $n=10,000$. Fits for all three batch sizes tested are nearly identical for batch sizes $b=1,5$, and 10

Table 7.2 . 95\% confidence intervals for the mean and variance for the number of observations truncated for batch sizes $b=1,5$, and 10 for run length $n=10,000$

\begin{tabular}{|l|r|r|r|}
\hline \multicolumn{1}{|c|}{ Observations Truncated } & Batch Size 1 & \multicolumn{1}{c|}{ Batch Size 5 } & Batch Size 10 \\
\hline Sample Mean & 146.9190 & 152.5950 & 157.8500 \\
\hline Upper limit & 147.0289 & 155.2085 & 159.1441 \\
\hline Lower limit & 146.8091 & 151.9817 & 156.5159 \\
\hline Sample Std Dev & 1.7708 & 9.8858 & 21.1764 \\
\hline Upper limit & 1.8520 & 10.5571 & 22.1477 \\
\hline Lower limit & 1.6964 & 9.4688 & 20.2872 \\
\hline
\end{tabular}

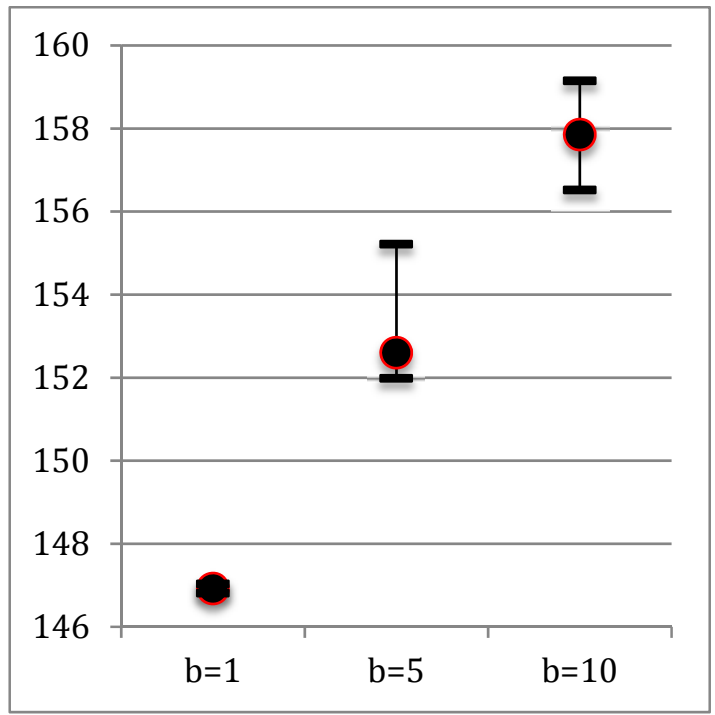

(a)

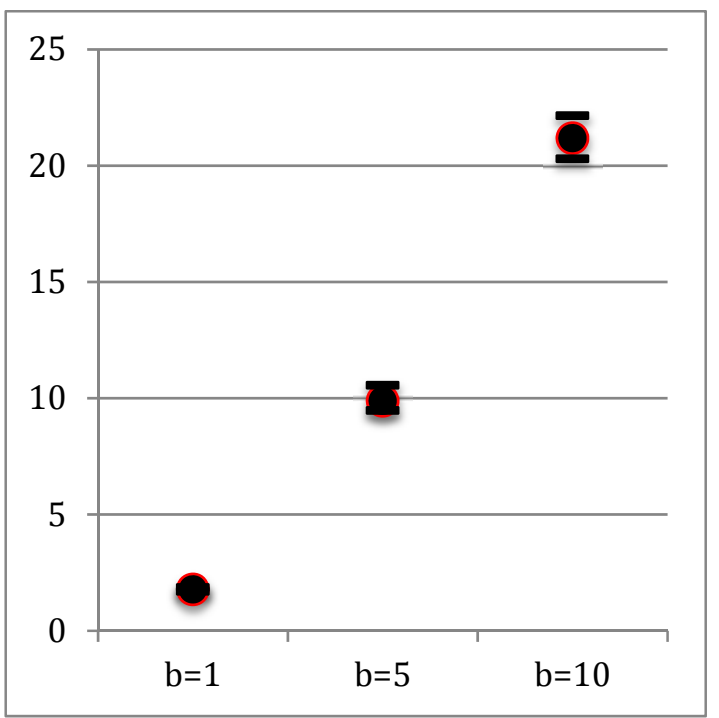

(b)

Figure 7.6 95\% confidence intervals for (a) the MSER mean and (b) the standard deviation in the MSER truncation point for run lengths $n=10000$ and batch sizes $b=1,5,10$ 
The scatter diagram in Figure 7.7, the frequency histogram in Figure 7.8, and the correlation coefficients in Table 7.3 illustrate the relationship between the truncated means and the corresponding total number of observations truncated. We conclude that for this instance

(4) increasing batch sizes increases both the variance and spread of the truncated observations, without systematically affecting the accuracy of the estimated mean,

(5) the mean estimate is uncorrelated with the number of observations truncated for all the batch sizes, and

(6) the success of a truncation procedure in terms of the accuracy of the estimate cannot be imputed from the truncation point alone.

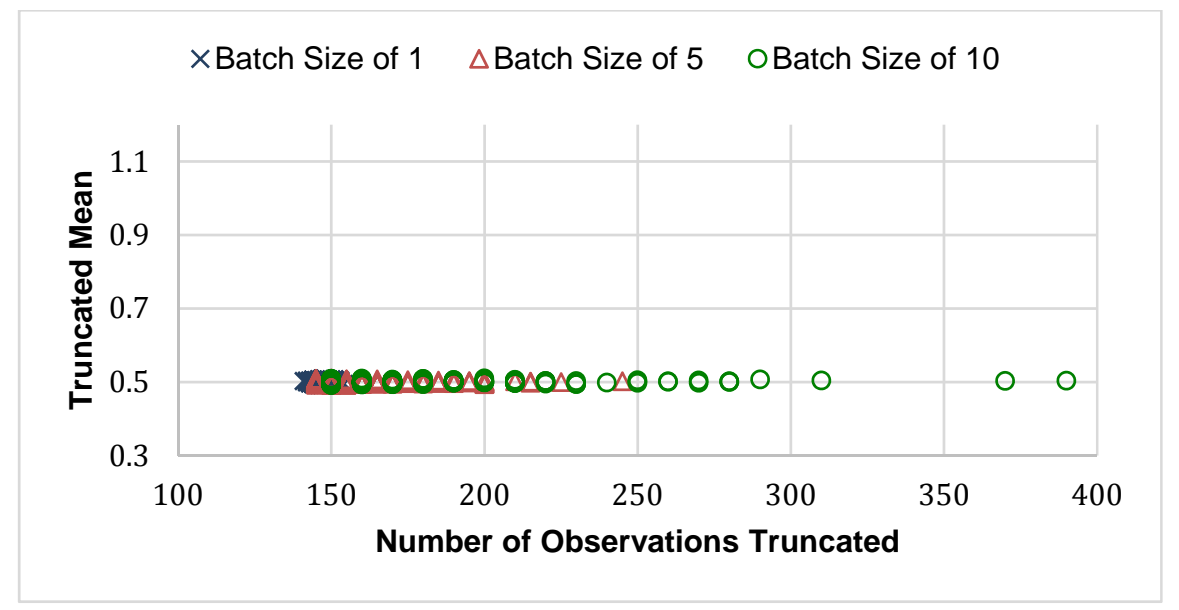

Figure 7.7 Scatterplots of the truncated mean vs. the number of observations truncated for batch sizes $b=1,5$, and 10 for run length $n=10,000$ 


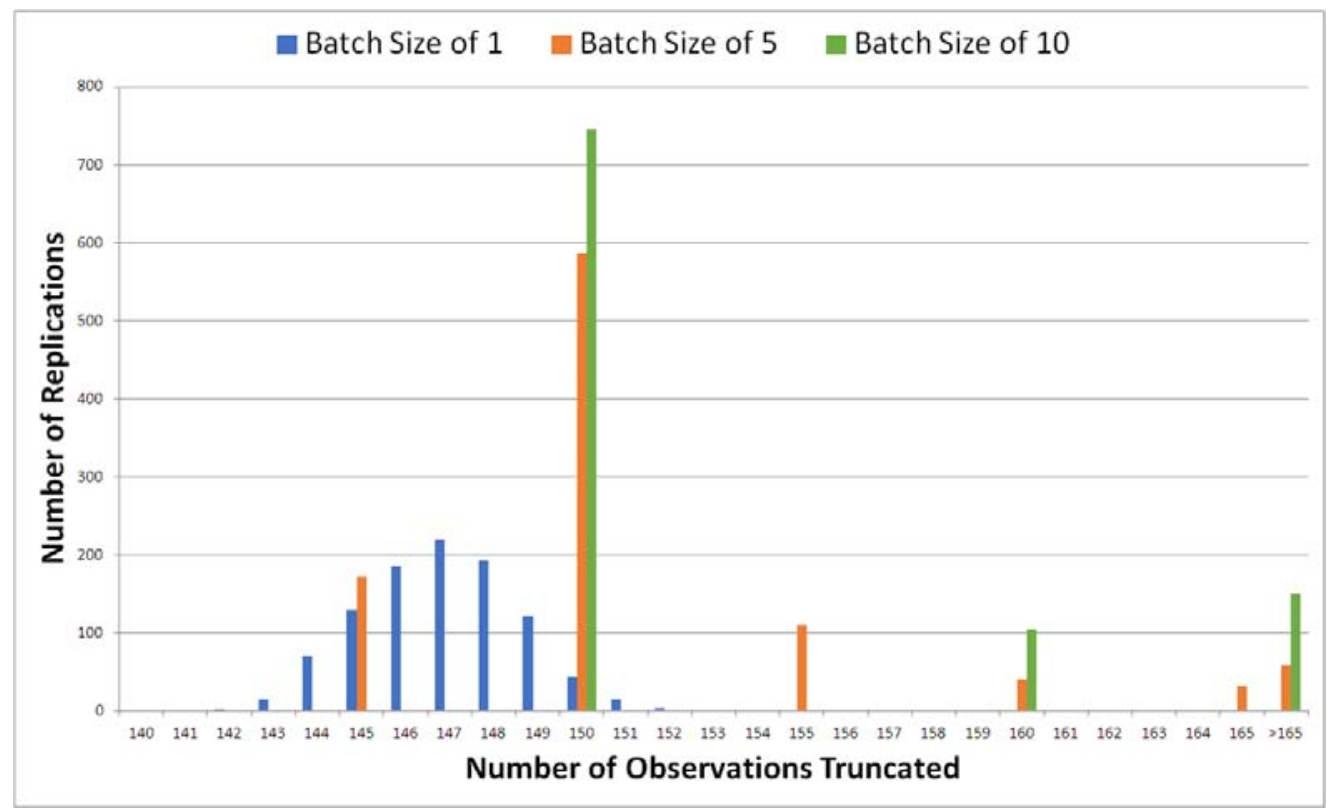

Figure 7.8 Frequency distribution of the total number of observations truncated as a function of batch size for batch sizes $b=1,5$, and 10 for run length $n=10,000$

Table 7.3 Correlation between the truncated mean and the number of observations truncated for batch sizes $b=1,5$, and 10 for run length $n=10,000$.

\begin{tabular}{cccc} 
Linear Correlation Table & Trunc Batch=1 & Trunc Batch=5 & Trunc Batch=10 \\
\hline \hline Mean Batch $=\mathbf{1}$ & -0.054 & -0.011 & 0.075 \\
Mean Batch $=\mathbf{5}$ & -0.046 & -0.015 & 0.072 \\
Mean Batch $=\mathbf{1 0}$ & -0.045 & -0.014 & 0.076
\end{tabular}

As can be seen in Figure 7.8, increasing the batch size tends to increase the number of observations truncated in part because of the increased granularity — with larger batches there are fewer candidate truncation points to consider. But this trend also reflects the difference between the "true" and MSER truncation points. To further explore, consider output for replication 958. The MSER truncation points for batches of $b=1,5$, and 10 are $d^{*}=148,190$, and 590 respectively, where 590 is the largest truncation point observed across all replications of with all batch sizes. 
The batch means and MSER statistic for that replication are plotted against the first 500 observations in Figure 7.9. Figure 7.10 provides a closer look these data for $b=10$ on the range $t \in[100,1500]$, i.e., batches 10 through 150 . The mean for batch number 59 is 0.1524185 , which is nearly 4 standard deviations below the steady-state mean. Given the length of the reserved sequence for this truncation point (941 batches), MSER can improve the estimate of the mean by deleting this observation.

Finally, we note that in general there are two effects of batching. First, the sequence is whitened, decreasing any serial correlation in the batch means. Second, the sequence is smoothed, altering the form of the steady-state distribution, decreasing any skew, and making the resulting distribution more nearly normal. These effects are consequences of the Central Limit Theorem.
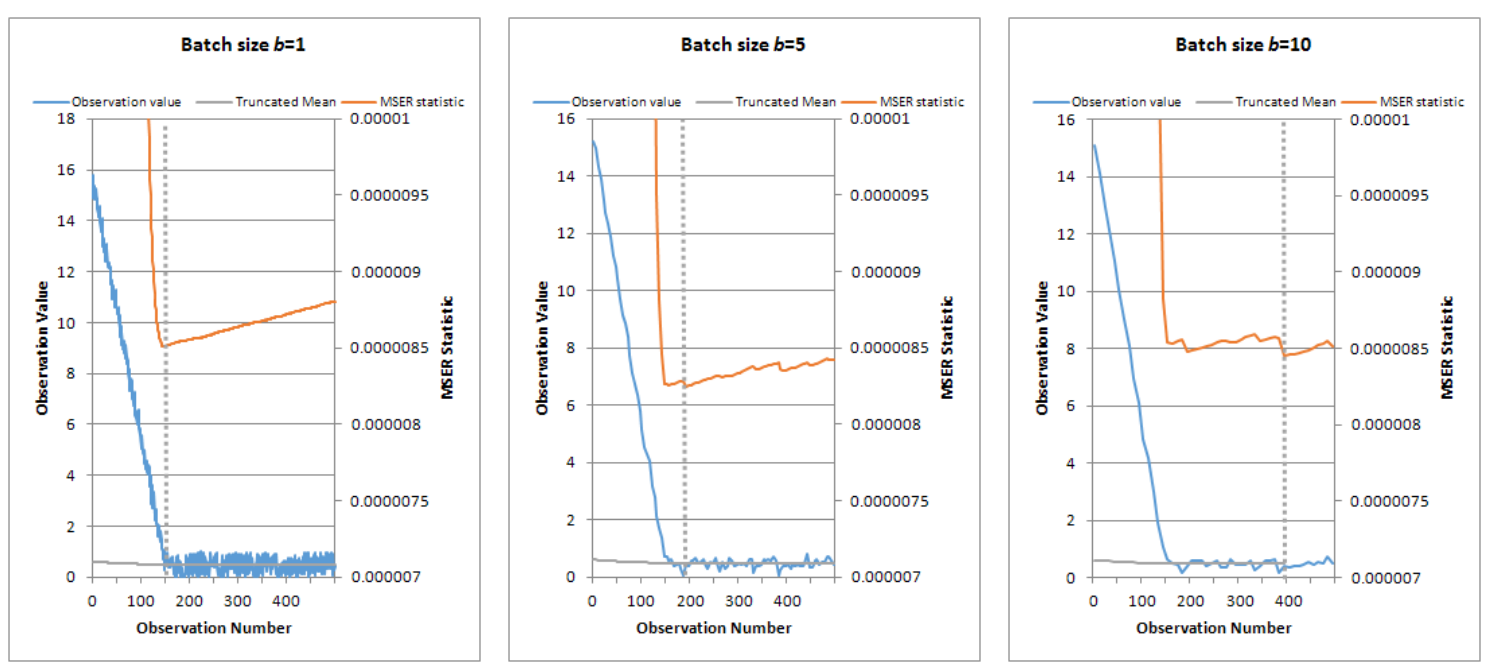

Figure 7.9 Batched means and MSER statistic for the first 500 observations for replication 958 for batch sizes $b=1,5$, and 10 for run length $n=10,000$ 


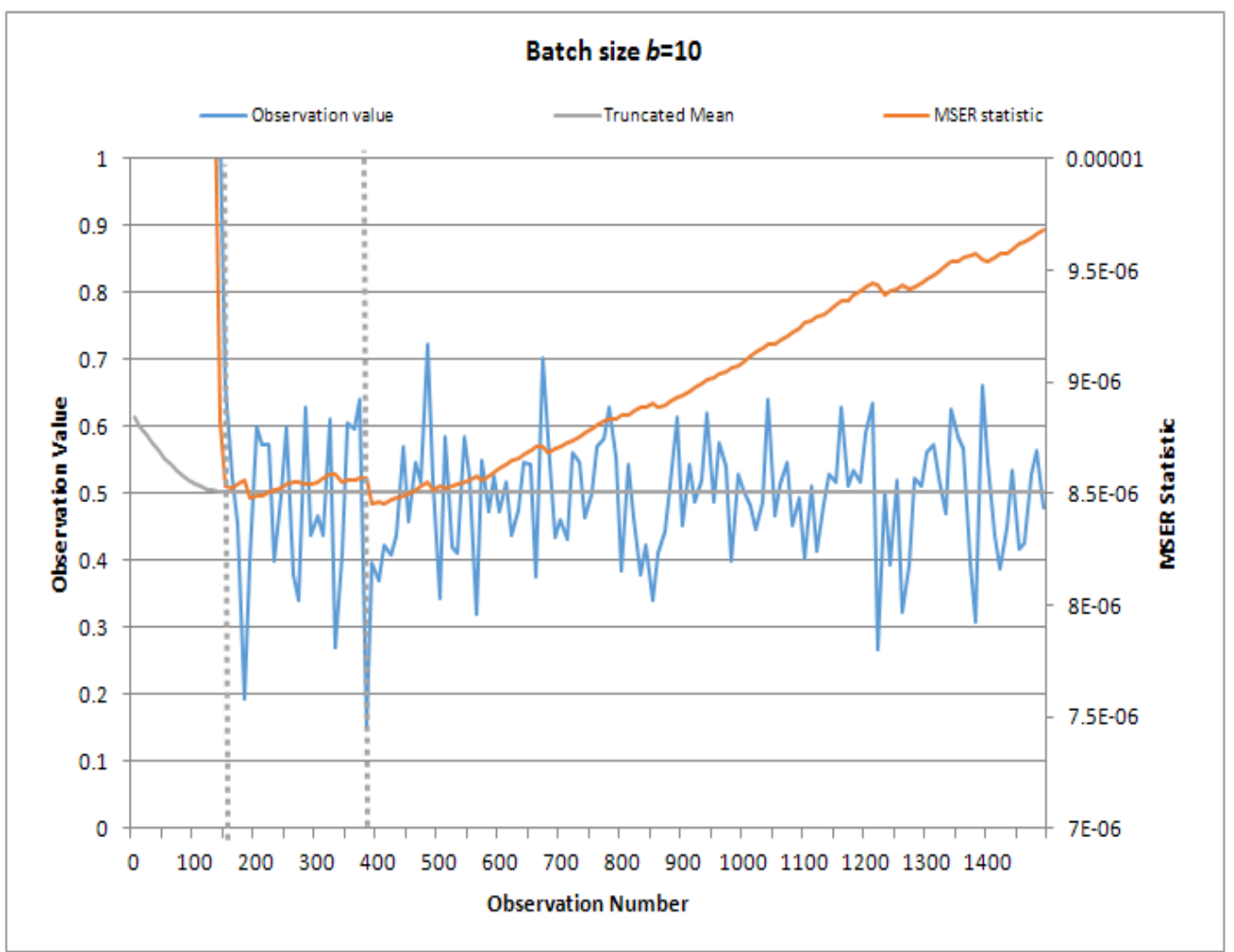

Figure 7.10 Batch means and MSER statistic for the first 1500 observations for replication 958 for batch sizes $b=10$ and run length $n=10,000$

For Model 1 specifically, the whitening effect is moot, since the steady-state sequences are already white without batching. However, the second effect can be further characterized. Remember that the batched output is the random variable

$$
Z_{j}=(1 / b) \sum_{p=1}^{b} X_{b(j-1)+p}=(1 / b) Y_{p}
$$

The $X$ 's in this case are independent and identically distributed $\operatorname{UNIF}(0,1)$. Therefore the sums $Y$ are independent random variables from an Irwin-Hall distribution (also known as the uniform sum distribution) of degree $b$ with density function

$$
f_{Y}(y ; b)=\frac{1}{2(n-1) !} \sum_{k=0}^{b}(-1)^{k}\left(\begin{array}{l}
b \\
k
\end{array}\right)(y-k)^{n-1} \operatorname{sgn}(y-k)
$$


This random variable has support $[0, b]$, mean $b / 2$, variance $b / 12$, and skew 0 .

Scaling by the constant $b$, the distribution of the batched observations is therefore

$$
f_{Z}(z ; b)=\frac{1}{2 b(n-1) !} \sum_{k=0}^{b}(-1)^{k}\left(\begin{array}{l}
b \\
k
\end{array}\right)(y-k)^{n-1} \operatorname{sgn}(y-k)
$$

with support $[0,1]$, mean $1 / 2$, variance $1 / 12 b$, and skew 0 . Note that the support, mean, and skew are constant, while the variance is inversely proportional to the batch size. This density function is a symmetric, piecewise-polynomial spline. Special cases include $\operatorname{UNIF}(0,1)$ for $b=1$ and $\operatorname{TRI}(0,0.5,1)$ for $b=2$. As $b$ increases, the distribution approximates $\operatorname{NORM}\left(\frac{1}{2}, \frac{1}{\sqrt{12 b}}\right)$. This is illustrated for replication 958 in Figure 7.11, which shows the estimated steady-state distribution (after truncation), and Table 7.4, which provides summary statistics, for $b=1,5$, and 10 .
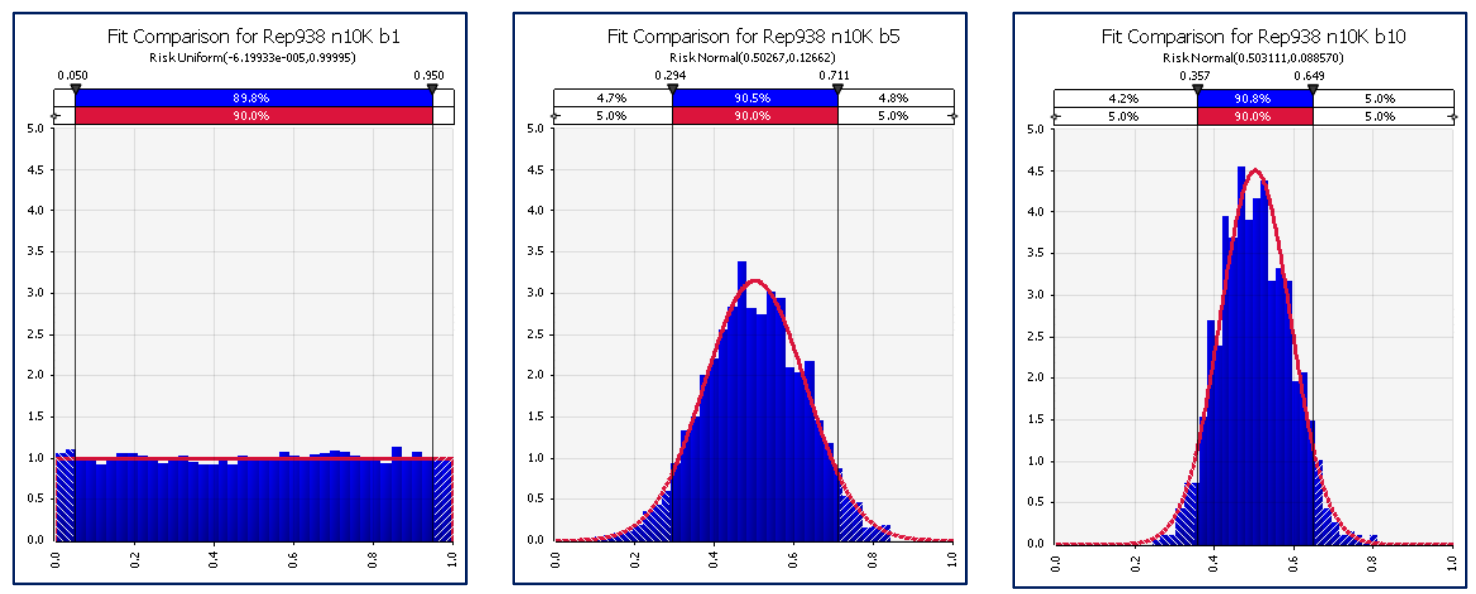

Figure 7.11 Estimated steady-state distribution (after truncation) for a single representative

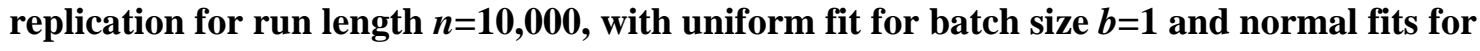
batch sizes $b=5$ and 10 
Table 7.4 Summary statistics for the steady-state data given in Figure 7.11.

\begin{tabular}{|l|r|r|r|}
\hline & \multicolumn{1}{|c|}{$b=1$} & \multicolumn{1}{c|}{$b=5$} & \multicolumn{1}{c|}{$b=10$} \\
\hline Mean & 0.50246 & 0.50267 & 0.50511 \\
\hline Mode & $\approx 0.85641$ & $\approx 0.55255$ & $\approx 0.48050$ \\
\hline Median & 0.50874 & 0.50217 & 0.50017 \\
\hline Std Dev & 0.28949 & 0.12662 & 0.08857 \\
\hline Variance & 0.085804 & 0.016055 & 0.007845 \\
\hline$b^{*}$ Var & 0.085804 & 0.080165 & 0.078446 \\
\hline $1 / 12$ & 0.085555 & 0.085555 & 0.085555 \\
\hline Skewness & -0.0271 & -0.0582 & 0.1771 \\
\hline Kurtosis & 1.7926 & 2.8118 & 2.8455 \\
\hline
\end{tabular}

Interestingly, we can show that for this model the MSER statistic itself is independent of the batch size and decreases linearly as a function of run length. Let $n b=n / b$ be the number of batches in the reserved sequence and let $\operatorname{Var}_{b}=1 / 12 b$ be the variance for this sequence. The MSER statistic is then

$$
\operatorname{MSER}(n, b)=\operatorname{Varb} / n b=(1 / 12 \mathrm{~b}) /(n / b)=1 / 12 n .
$$

\subsubsection{Results: Batch size effects for short runs}

The preceding results suggest that mean estimates are both empirically and theoretically insensitive to batch size for Model 1 . These results are derived using a run length that is several orders of magnitude longer than the state-transient sequence, however, and one might anticipate that results for much shorter runs will differ. To explore this intuition, we repeated the analysis with run lengths of $n=175,200,300$, and 500 observations. 
Results are displayed in Figures 7.12-7.15. The overall effect of reducing runs lengths is to rotate the centerline of the scatter plots from one that is essentially horizontal to one that is increasingly vertical, implying an increasingly negative correlation between the number of observations truncated and the run length. The average and spread of the number of observations deleted both decrease; the average and spread of the estimated steady-state mean both increase. The strength of this effect increases as the batch size increases.

For $n=500$, the larger batch sizes continue to yield additional truncation beyond the $[140,150]$ range in some instances, for the same reasons illustrated earlier. The difference is that the largest truncation points are now for $b=5$, instead of $b=10$.

For $n=500$ and $n=200$, the results are essentially identical. Nearly all of the truncation points are on the anticipated range $[140,150]$, none are less than 140 , and comparatively few are greater than 150. The smallest truncation points are now associated with the largest batch sizes - a complete reversal from the results obtained with long runs. Indeed, for $b=5$ almost all of the truncation points are 145 or 150 ; for $b=10$ the truncation point is 140 for all 1000 replications.

For $n=175$, the effects of very short sequences are most pronounced. The truncation points for $b=1$ rarely exceed 150 . For $b=5$ all of the truncation points are 145 . For $b=10$ almost all are 140, with a very few at 150 .

Note that for $n=175$ and $b=10$, all of the estimates suffer from the effect of bias, some acutely. This is because algorithm sees very little data-a total of 17 batches, only two of which are in steady state. Depending on the realization of the noise process, MSER bases its estimate on either two or three batch means. For those few replications where a third 
(nonrecurrant) observation is retained, estimates are about twice the steady-state mean. For this combination of batching and run length, correlation between the truncation point and error in the mean estimate is strongest, with greater truncation leading to greater error in the mean estimates.

All of the effects noted are consequences of the relative weight MSER gives the sample size of the reserved sequence $\left(n_{b}-d\right)$ and the residuals of the observations $\left(x_{t}-\bar{x}\right)$. For larger samples, MSER is relatively less sensitive to the sample size; for small samples MSER is relatively more sensitive to the sample size. Therefore, we conclude for Model 1 that

(1) to the degree that batching reduces the effective sample size, it is not recommended for small samples and provides no discernable benefit for large samples, and

(2) even with very little steady-state data, the MSER-indicated truncation points are themselves very reasonable and indeed optimal in terms of the mean estimates for most cases.

(3) the choice of $d_{\text {max }}$ is a binding concern only if $(n-d) / n$ is close to 1 - the choice of $n$ is likely dominated by the need for estimates with acceptable accuracy and precision.

Finally, and perhaps most importantly, all of the results above use all of the simulation replications, which is equivalent to setting the maximum truncation point at $d_{\max }=n$. What happens if, instead, we impose the recommend threshold $d_{\max }=n / 2$ and discard replications for which $d^{*}>d_{\max }$ ? We can see from the results that (1) for $n=10,000$ and 500 , the threshold has no effect on the results; (2) for $n=300$, however, a significant number of 
replications are discarded for $b=5$ and 10; and (3) for $n=200$ and 175, all of the replications will be discarded! We conclude that, for this model,

(4) the $d^{*} \leq d_{\max }=n / 2$ threshold provides significant protection against estimation errors resulting from run lengths that are too short' without over-truncation of replications with adequate run lengths. For run lengths that are approximately the same as ideal truncation point, however, the protection may be inadequate. A modestly lower threshold would be preferred. 


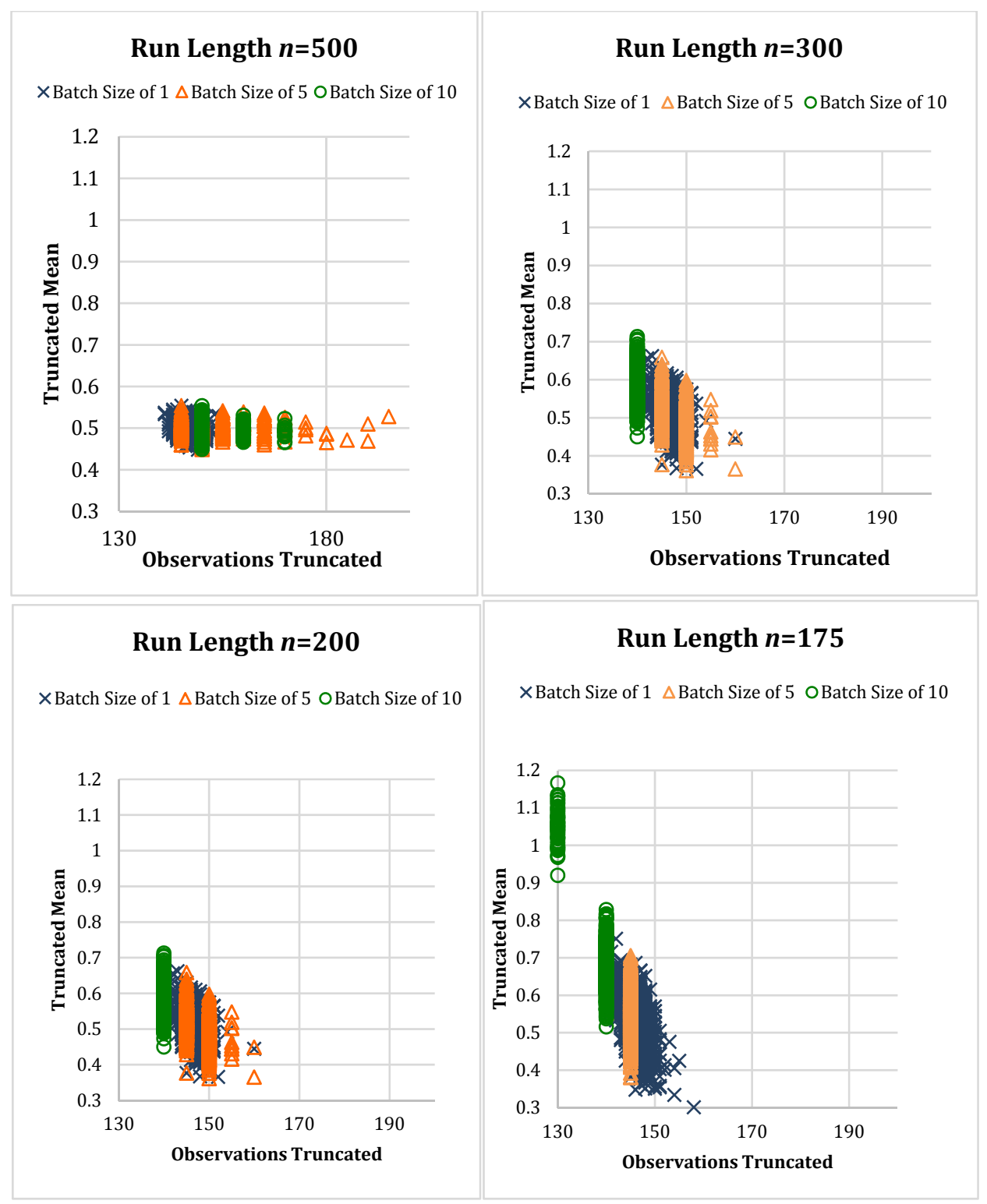

Figure 7.12 Scatterplots of the truncated mean vs. the number of observations truncated batch sizes $b=1,5$, and 10 for run lengths $n=175,200,500$, and 500 

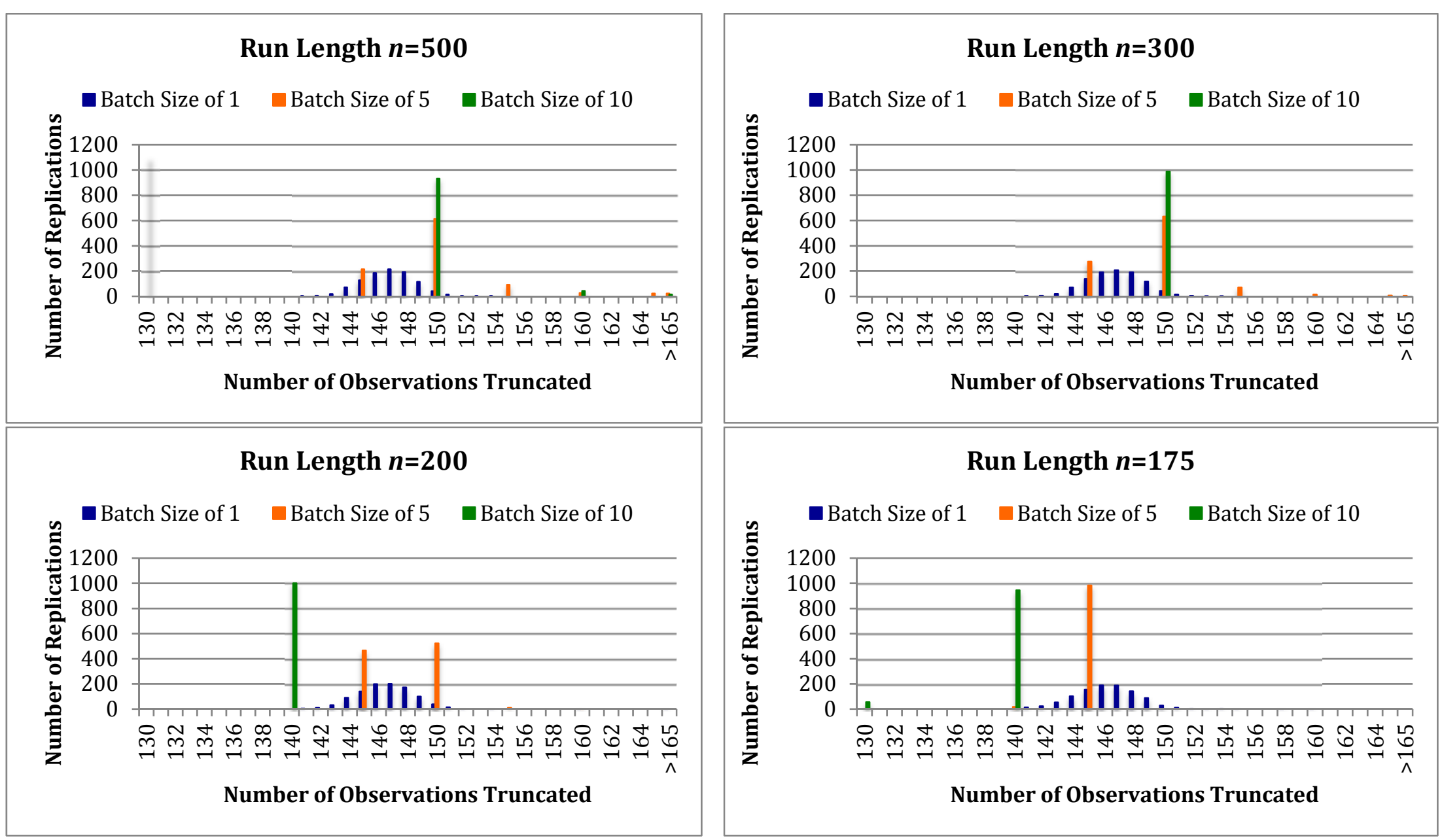

Figure 7.13 Frequency distribution of the total number of observations truncated as a function of batch size for batch sizes $b=1,5$, and 10 for run lengths of $n=175,200,500,500$ 
$n=175$
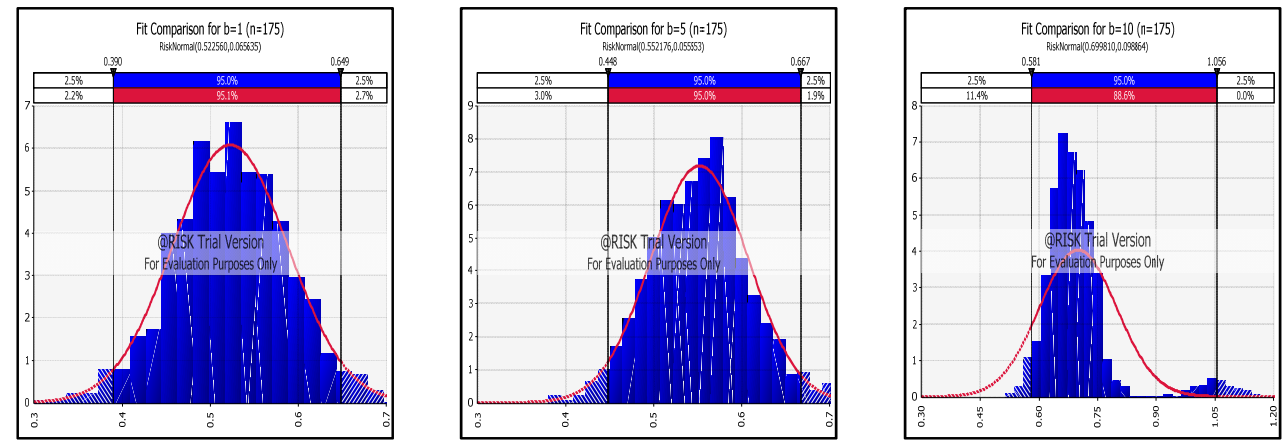

$n=200$
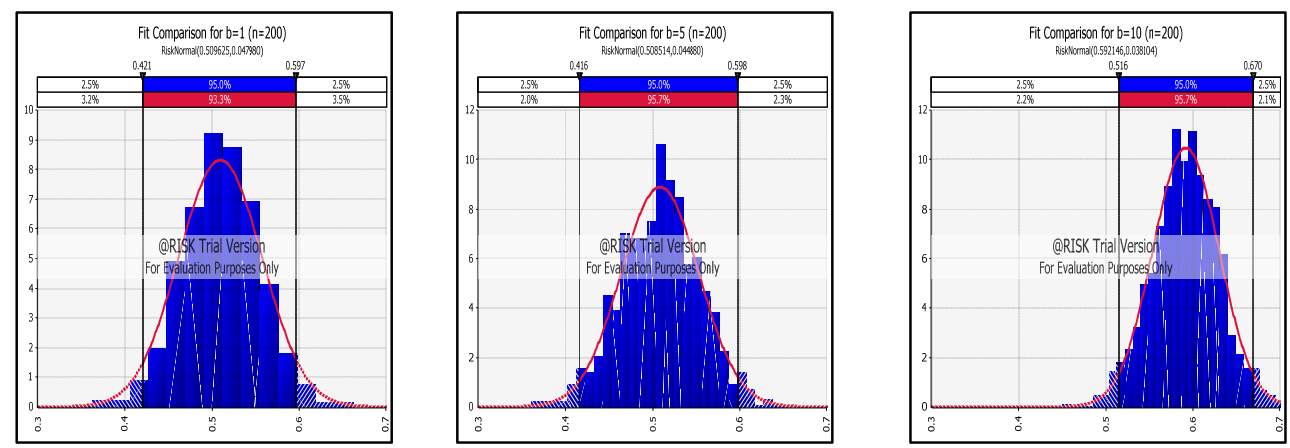

$n=300$
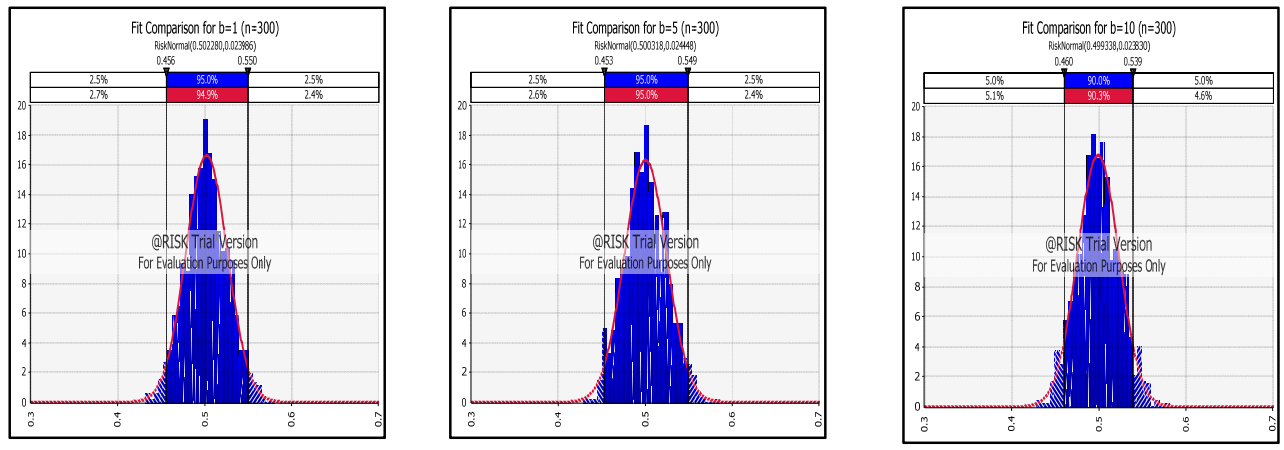

$n=500$
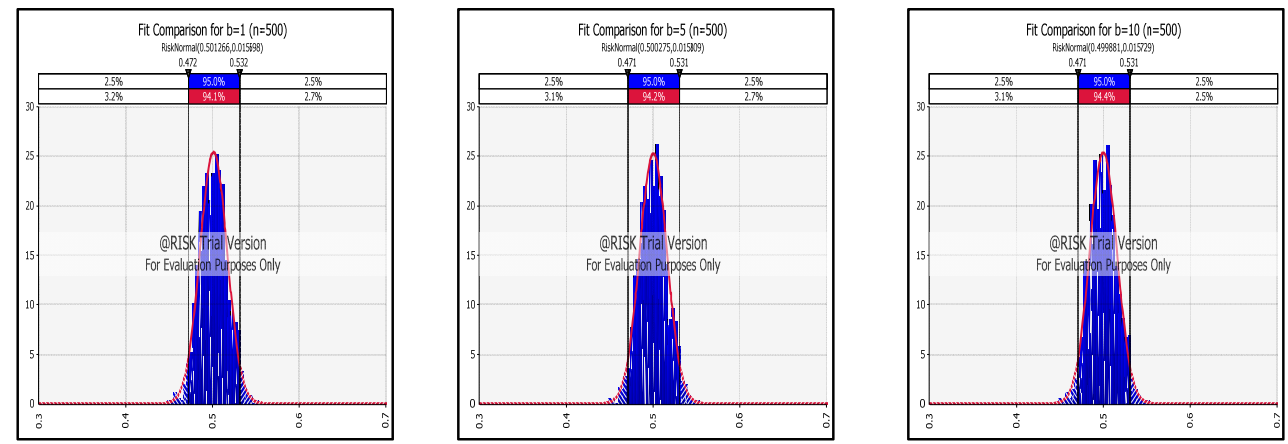

Figure $7.1495 \%$ confidence intervals of the mean for the truncated mean output as a function of batch size for batch sizes $b=1,5$, and 10 for run lengths of $n=175,200,300,500$ 
$n=175$

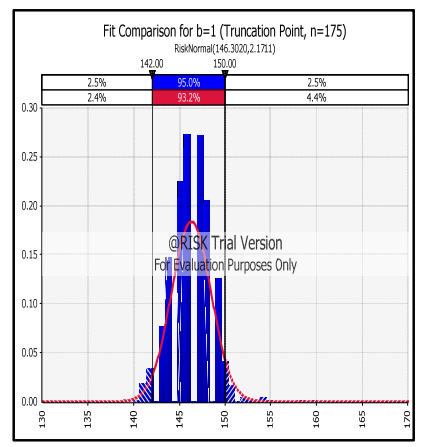

$n=200$

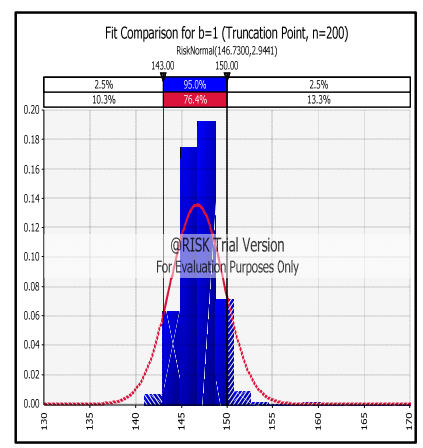

$n=300$

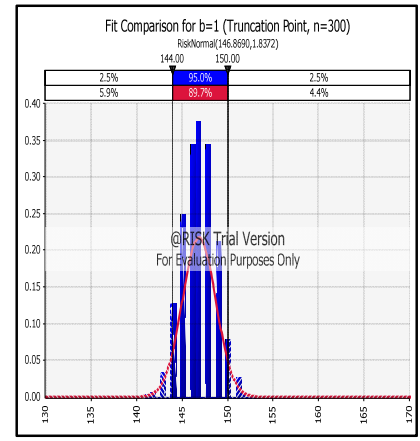

$n=500$

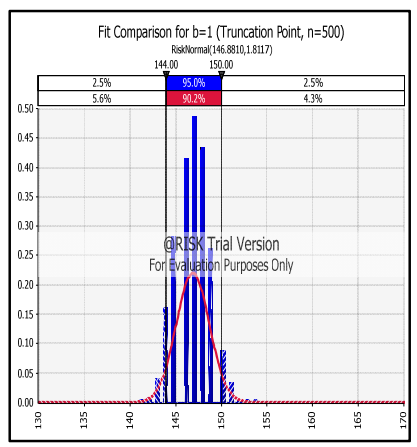

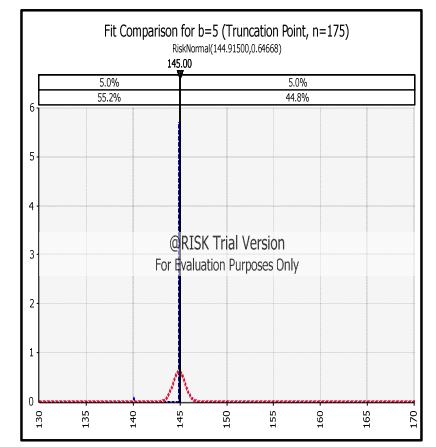
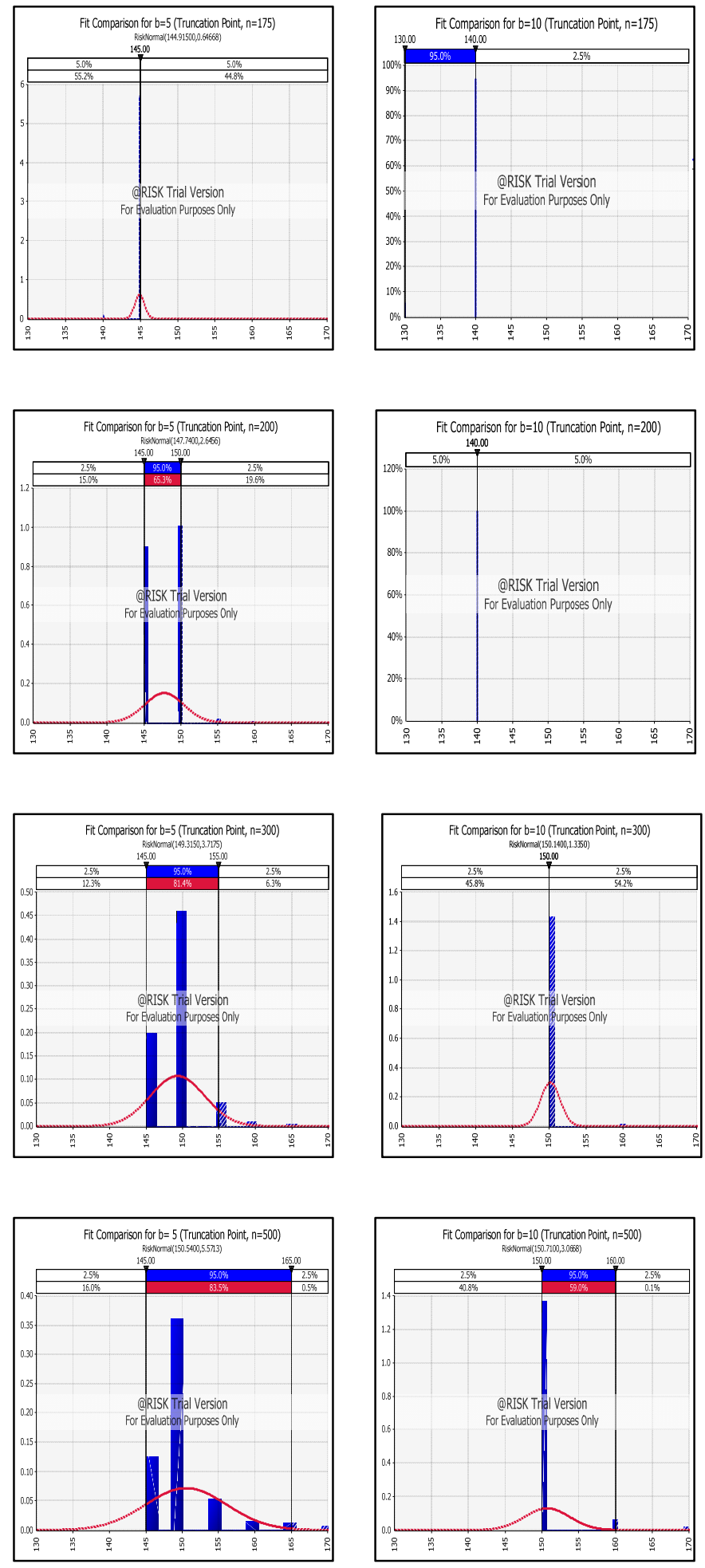

Figure 7.15 95\% confidence intervals for the mean for the number of observations truncated for batch sizes $b=1,5$, and 10 for run lengths of $n=175,200,300,500$ 


\subsubsection{Results: Overlapping Batch Means}

We applied OBM to Model 1 with run length $n=500$ for batch sizes $b=10,35,75$, and 150 observations. Figure 7.16 shows output for four representative replications, one at each batch size. Table 7.5 and Figure 7.17(a) compares the $95 \%$ confidence intervals on the truncated mean for each of the OBM estimates with the truncated mean for nonoverlapping approach with the single batch size $b=10$. All of the interval estimates cover the expected value of 0.5 . None of the differences among the mean estimates is significant at the 0.05 level, however the results suggest that OBM may be the superior approach, particularly for modestly sized batches. Model 1 simply isn't challenging enough to draw a definitive conclusion.
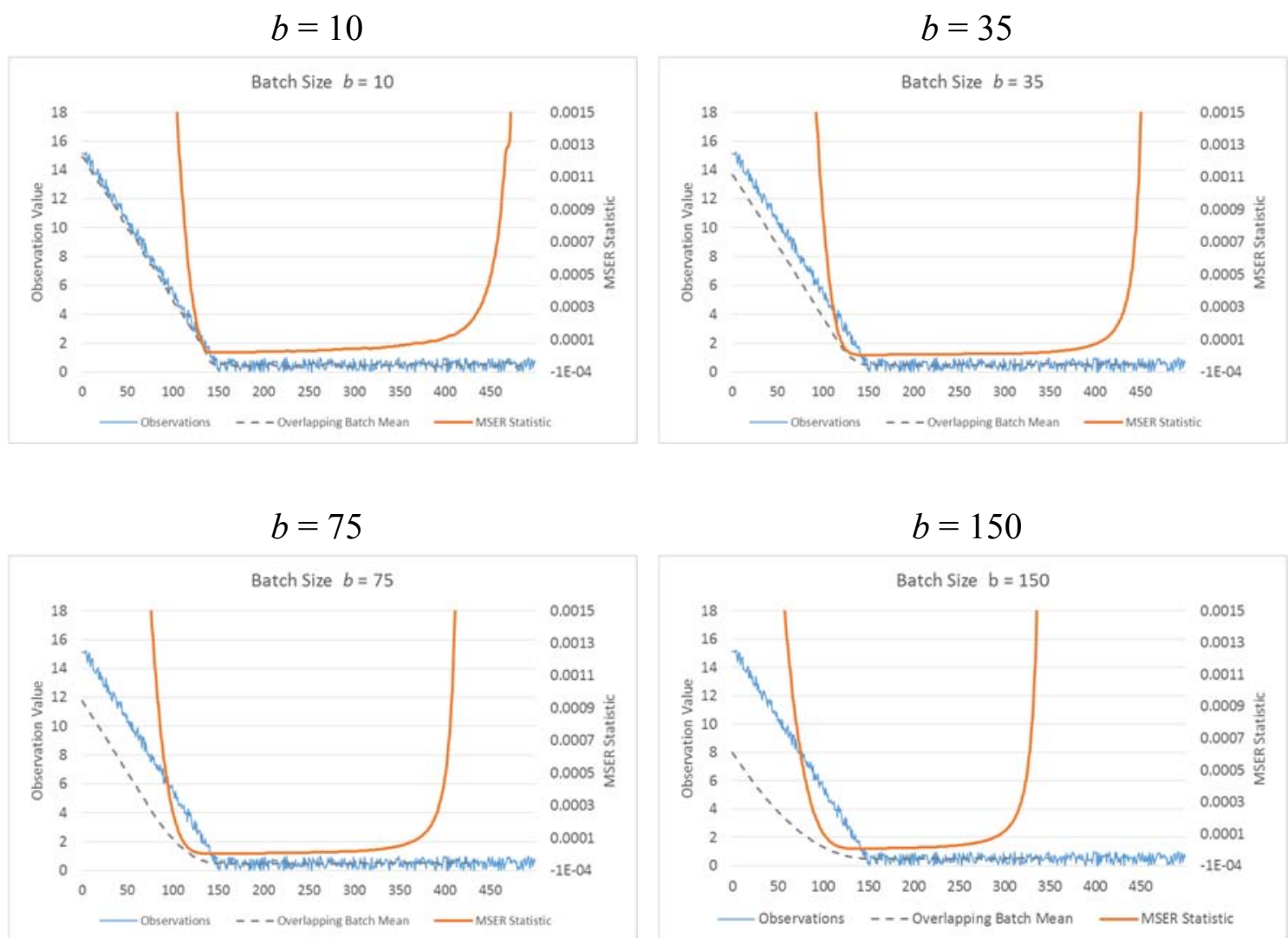

Figure 7.16 Representative Output, Overlapping Batch Mean, and MSER Statistic of Model $1(b=10,35,75$, and 150) 
Table 7.5 95\% confidence intervals for Model 1 on the truncated mean and the standard deviation for overlapping and non-overlapping batches

\begin{tabular}{|c|c|c|c|c|c|}
\hline & \multicolumn{4}{|c|}{ OBM } & NOBM \\
\cline { 2 - 6 } & OBM $b=10$ & OBM $b=35$ & OBM $b=75$ & OBM $b=150$ & NOBM $b=10$ \\
\hline Sample Mean & 0.50005 & 0.49993 & 0.49965 & 0.50028 & 0.49934 \\
\hline Upper limit & 0.50104 & 0.50096 & 0.50071 & 0.50138 & 0.50082 \\
\hline Lower limit & 0.49906 & 0.4989 & 0.4986 & 0.49919 & 0.49786 \\
\hline Sample Std D & 0.01602 & 0.01661 & 0.017 & 0.01762 & 0.02383 \\
\hline Upper limit & 0.01675 & 0.01737 & 0.01777 & 0.01843 & 0.02492 \\
\hline Lower limit & 0.01534 & 0.01591 & 0.01628 & 0.01688 & 0.02283 \\
\hline
\end{tabular}

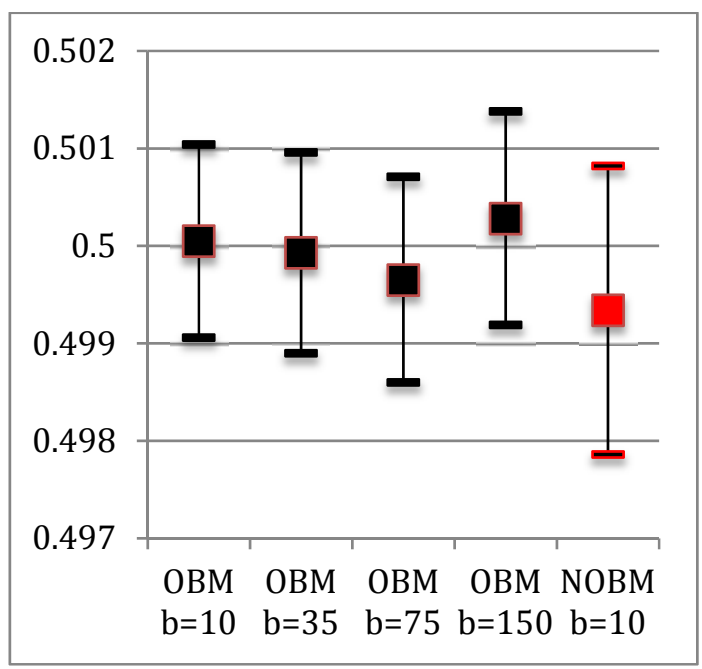

(a)

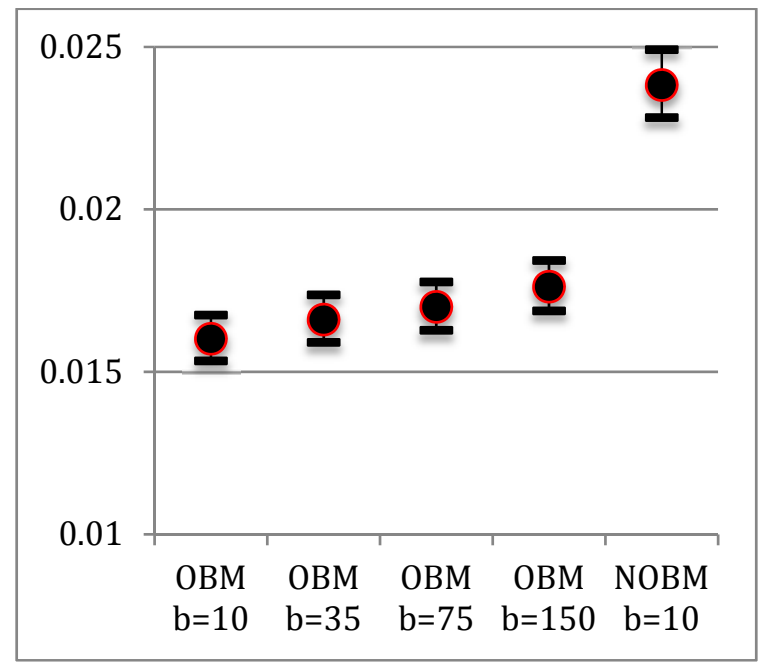

(b)

Figure 7.17 95\% confidence intervals for Model 1 on (a) the truncated mean for overlapping and non-overlapping batches and (b) the standard deviation for overlapping and non-overlapping batches

In contrast, Figure $7.17(\mathrm{~b})$ compares the $95 \%$ confidence intervals on the standard deviation in estimates for the same replications. While the differences among OBM estimates are not significant, OBM outperforms the non-overlapping approach in all cases. At least for this test case, OBM provides significantly greater precision in the estimate. This reflects the considerably larger sample size afforded by overlapping batches. 


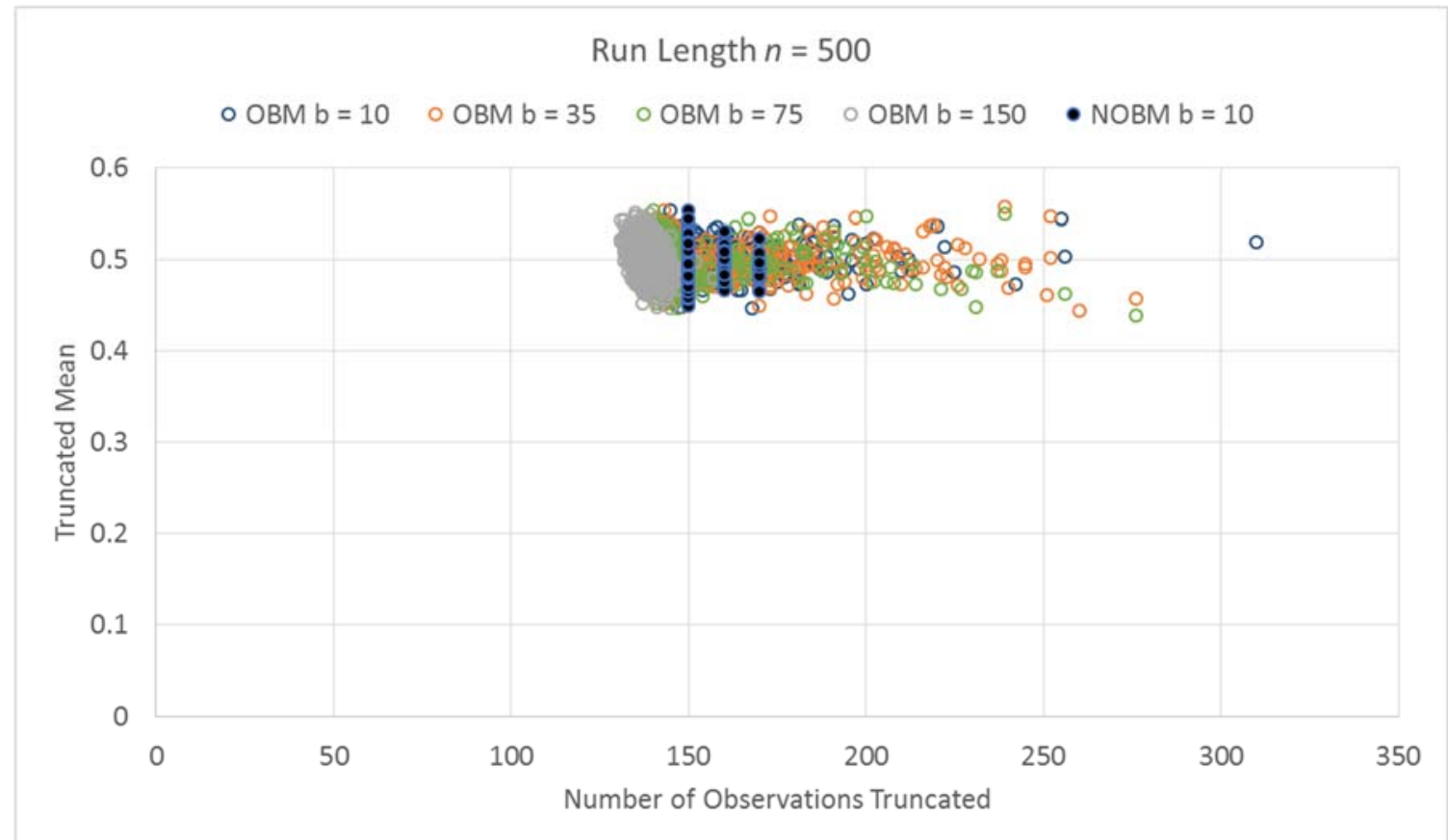

Figure 7.18 Scatterplots of the truncated mean vs. the number of observations truncated for OBM sizes b=10, 35, 75 and 150 for run length of $n=500$ for Model 1 (including NOBM batch size of 10)

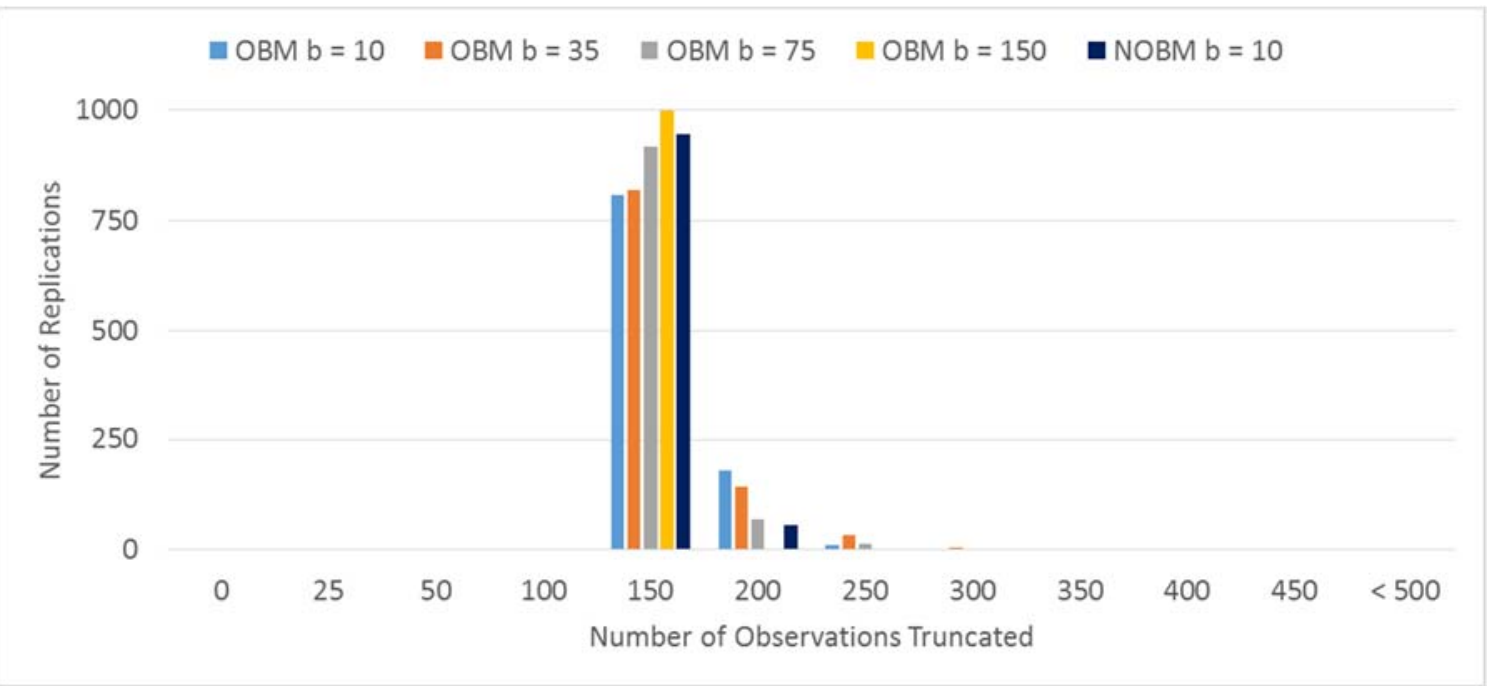

Figure 7.19 Frequency distribution of the number of observations truncated as a function of OBM sizes $b=10,35,75$, and 150 for run length of $n=500$ for Model 1 (including NOBM batch size of 10) 
Table 7.6. Correlation between the truncated mean and the number of observations truncated for OBM sizes $b=\mathbf{1 0}, \mathbf{3 5}, \mathbf{7 5}, 150$ for run length of $n=500$ for Model 1

\begin{tabular}{|l|l|l|l|l|}
\hline & Trunc Batch $=10$ & Trunc Batch =35 & Trunc Batch =75 & Trun Batch $=150$ \\
\hline Mean Batch $=10$ & -0.006655114 & 0.001668587 & 0.002054254 & -0.177174472 \\
\hline Mean Batch $=35$ & 0.000478214 & -0.0644325 & -0.087803961 & -0.282730256 \\
\hline Mean Batch $=75$ & -0.003975614 & -0.073941266 & -0.156501886 & -0.302717128 \\
\hline Mean Batch $=150$ & -0.027110964 & -0.046266742 & -0.076909778 & -0.346795267 \\
\hline
\end{tabular}

\subsubsection{Model 2: Waiting Time in an $M / M / 1$}

The second test model is an M/M/1 queue where our interest is in estimating the mean waiting-time in queue. This is the problem examined by Law (2015) and reexamined by White and Hwang (2015). We used this second model to ascertain the performance of MSER with the same batch sizes used in Model 1. In addition, we explored the sensitivity of estimates to changes in traffic intensity, the quotient

$$
\rho=\frac{\lambda}{\mu}
$$

where $\lambda$ is arrival rate, $\mu$ is service rate, and the expected waiting time in queue is

$$
E\left(W_{q}\right)=\frac{\lambda}{\mu(\mu-\lambda)} .
$$

The theoretical expected values of waiting time in queue for four different traffic intensities are summarized in Table 7.7. Clearly, the autocorrelation of successive observations of the waiting time is a function of the traffic intensity. For very low $\rho$, customers typically arrive at an empty and idle queue and do not have to wait for service. As the traffic intensity increases, customers experience increasingly longer waits on average. For $\rho \geq 1$, the demand for service exceeds the supply, the queue is unstable, and average waiting times become infinite. One might anticipate the effects of decreasing $\rho$ to be similar to those encountered by increasing the run length $n$. 
Table 7.7. Theoretical Waiting Time in Queue by Traffic Intensity

\begin{tabular}{lcccc}
\hline & \multicolumn{5}{c}{ Traffic Intensity $(\rho)$} \\
\cline { 2 - 5 } & 0.6 & 0.7 & 0.8 & 0.9 \\
\hline Waiting Time in Queue & 9 & 16.33 & 32 & 81 \\
\hline
\end{tabular}

\subsubsection{Results for Model 2 with traffic intensity of 0.90}

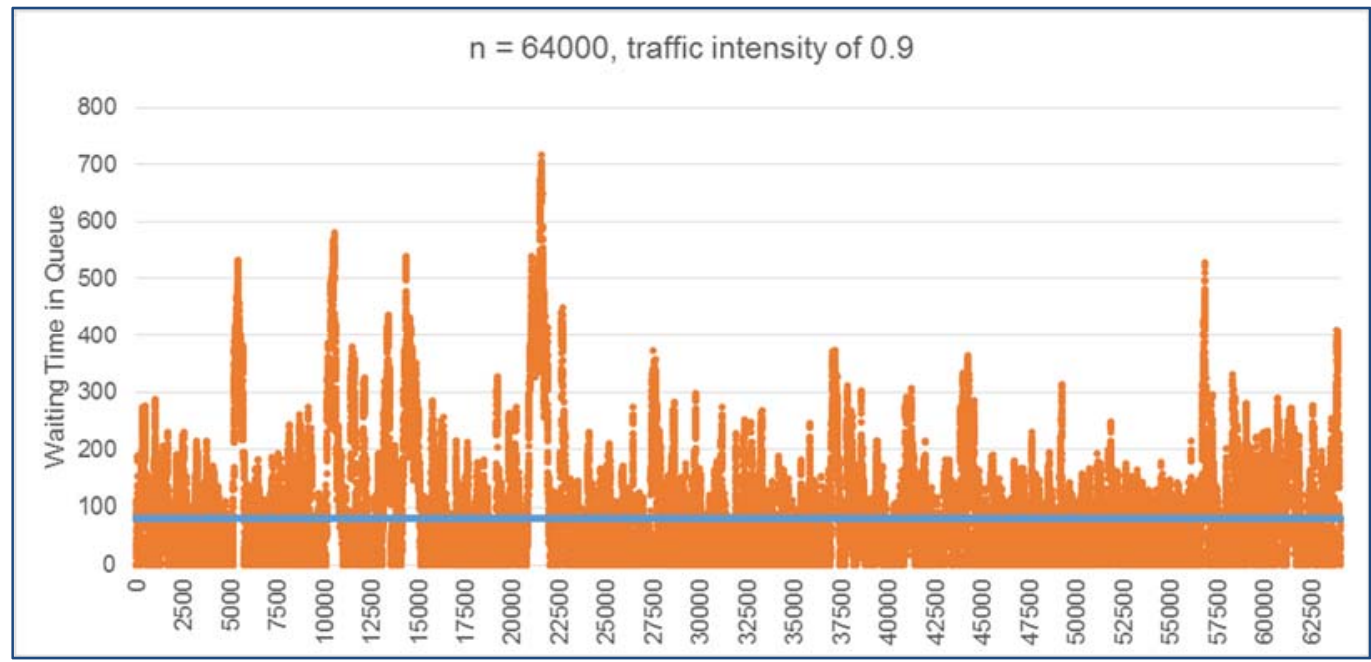

Figure 7.20 Representative Output of Waiting-Time in an $\mathrm{M} / \mathrm{M} / 1$ with Traffic Intensity of $0.9\left(n=64000\right.$; Blue line: $\left.E\left(W_{q}\right)=81\right)$

Figure 7.20 provides a typical output series from $\mathrm{M} / \mathrm{M} / 1$ queue with traffic intensity of $\rho=0.9$, initialized empty and idle $\left(x_{0}=0\right)$, for a run length of $n=64,000$. The output is said to be regenerative, in that it comprises independent cycles each beginning with a customer that experiences zero waiting. The system regenerates at when it returns to this same zero state and a new cycle begins. The duration of each cycle is random, as is the peak waiting time. Decreasing the traffic intensity will result in an increasing number of cycles and generally shorter peak waiting times.

We begin our analysis with the application of MSER to Model 2 and explore the combined impact of alternative batch sizes $(b=1,5$, and 10) and run lengths $(n=64,000$, $32,000,16,000,8,000,4,000,2000$, and 1,000). Our analysis follows the same steps 
introduced in the analysis of Model 1. For $\mathrm{M} / \mathrm{M} / 1$ with $\rho=0.9$, Figure 7.21 compares the confidence intervals on the sample means, Figure 7.22 compares the confidence intervals on the MSER truncation points, Figure 7.23 compares scatterplots of the truncated mean vs. the number of observations truncated, Table 7.8 provides the corresponding correlation coefficients, and Figure 7.24 compares the frequency histograms of the number of observations truncated.

Without truncation, the raw sample mean is 80.75 and insensitive to batch size. While the raw estimate does not provide coverage, the absolute estimation error is less than $1 \%$. The truncated is mean 79.39 and also insensitive to batch size. While the truncated estimate likewise does not provide coverage, the absolute estimation error is less than $2 \%$. The improvement result from truncation is very modest, but the difference in estimates is statistically significant at $\alpha=0.05$.

As the run length deceases, the raw estimate decreases more slowly than the truncated estimate. For run lengths of $n=8,000$ and below, the truncated means are exceptionally poor and far worse than the raw estimates. In general, the number of observations truncated decreases with as the run length decreases.

For the larger run lengths, we see that the mean estimates for this problem are quite good without truncation. Truncation improves on these estimates only for $n=64,000$ and then only very modestly. Truncation is contraindicated for smaller $n$. While there is negative trend in the estimated mean for larger truncation points, the correlation is negligible, but increasing in decreasing run length and increasing batch size. 

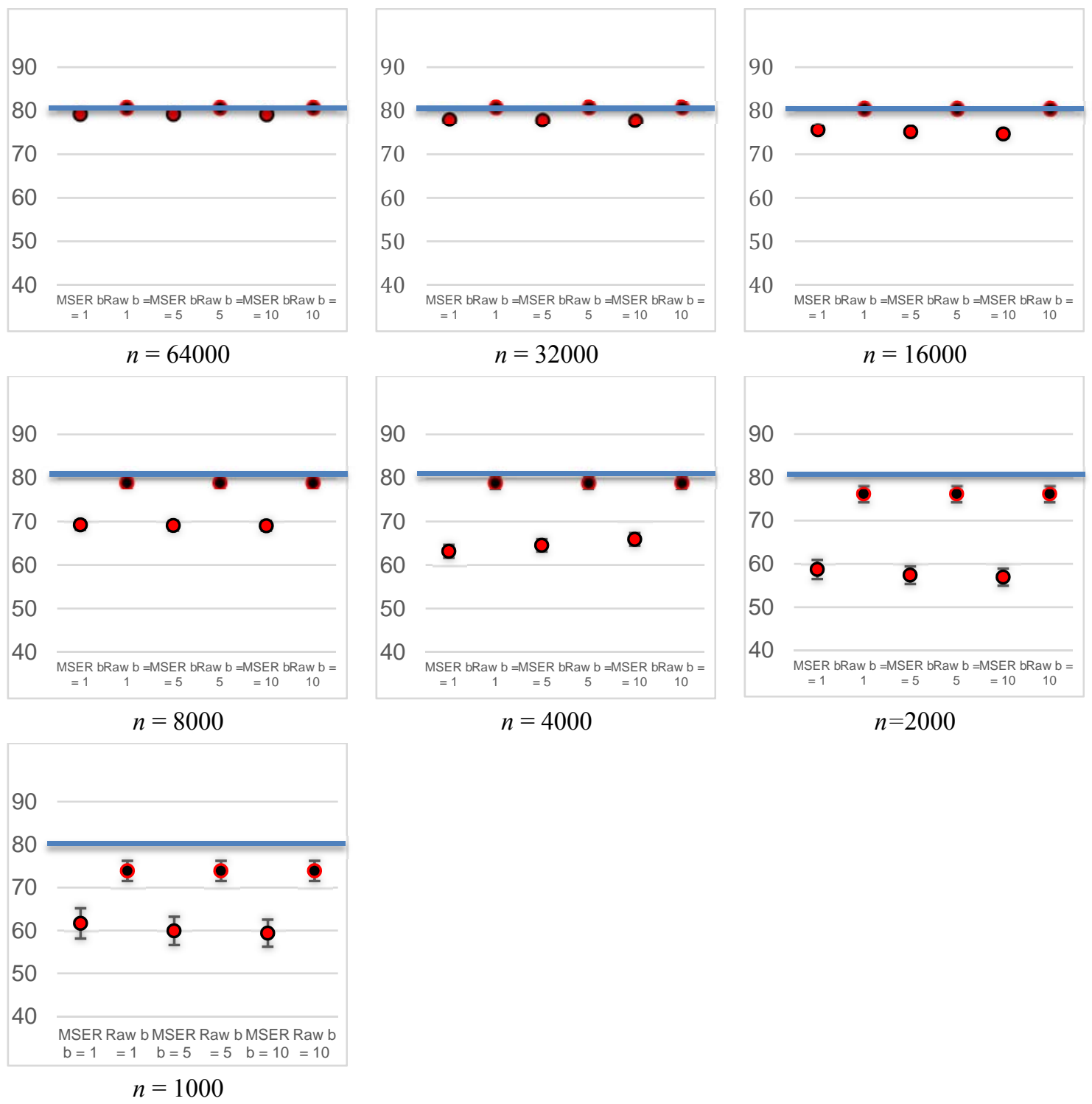

Figure $7.2195 \%$ confidence intervals for the mean and the truncated mean as a function of batch size $(b=1,5$, and 10) and run length $(n=1000,2000,4000,8000,16000,32000$, and 64000) for Model 2 with traffic intensity of 0.9 (Theoretical mean of 81, Blue line) 

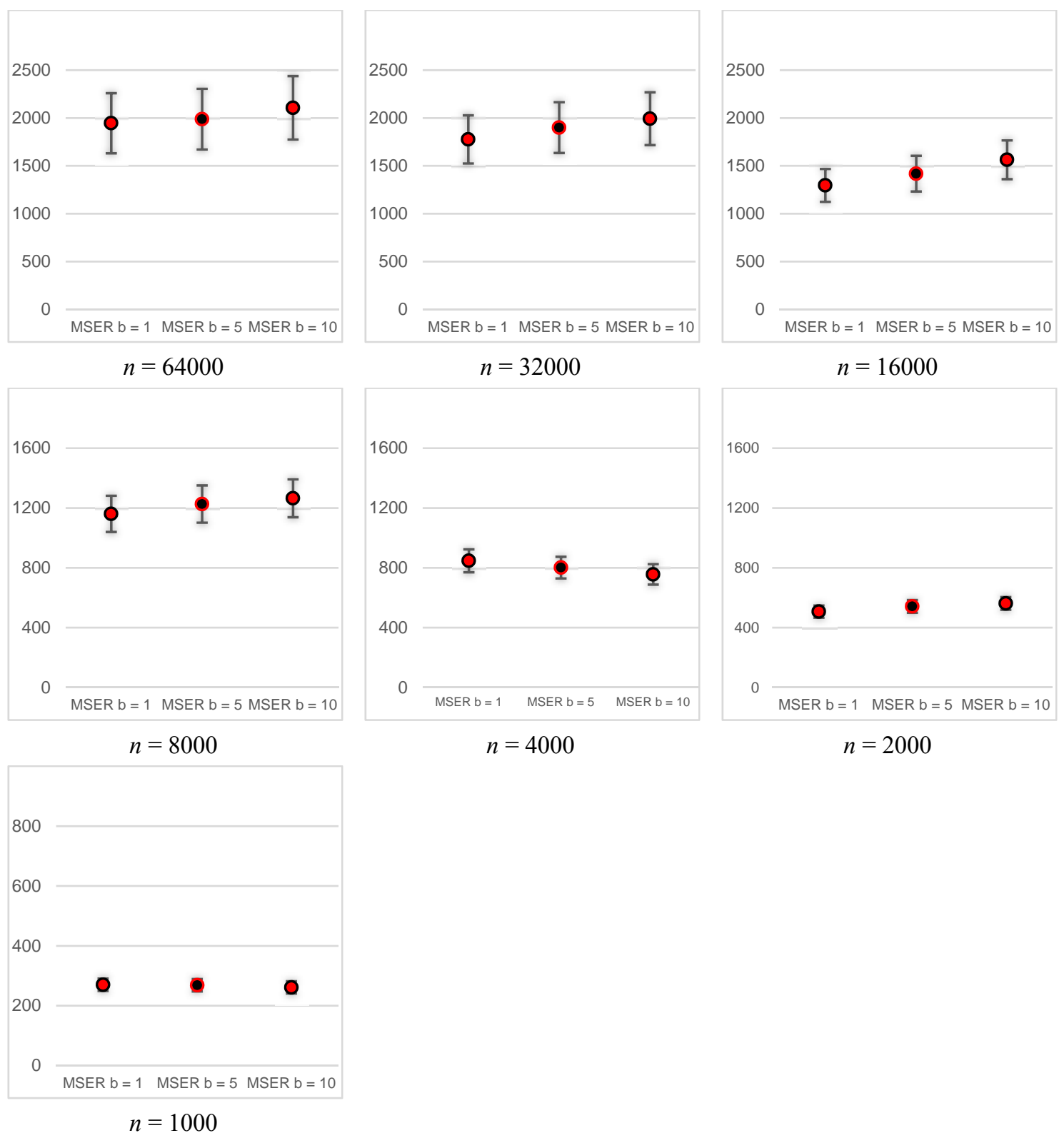

Figure $7.2295 \%$ confidence intervals for the mean number of observations truncated as a function of batch size $(b=1,5$, and 10) and run length $(n=1000,2000,4000,8000,16000$, 32000, and 64000) for Model 2 with traffic intensity of 0.9 

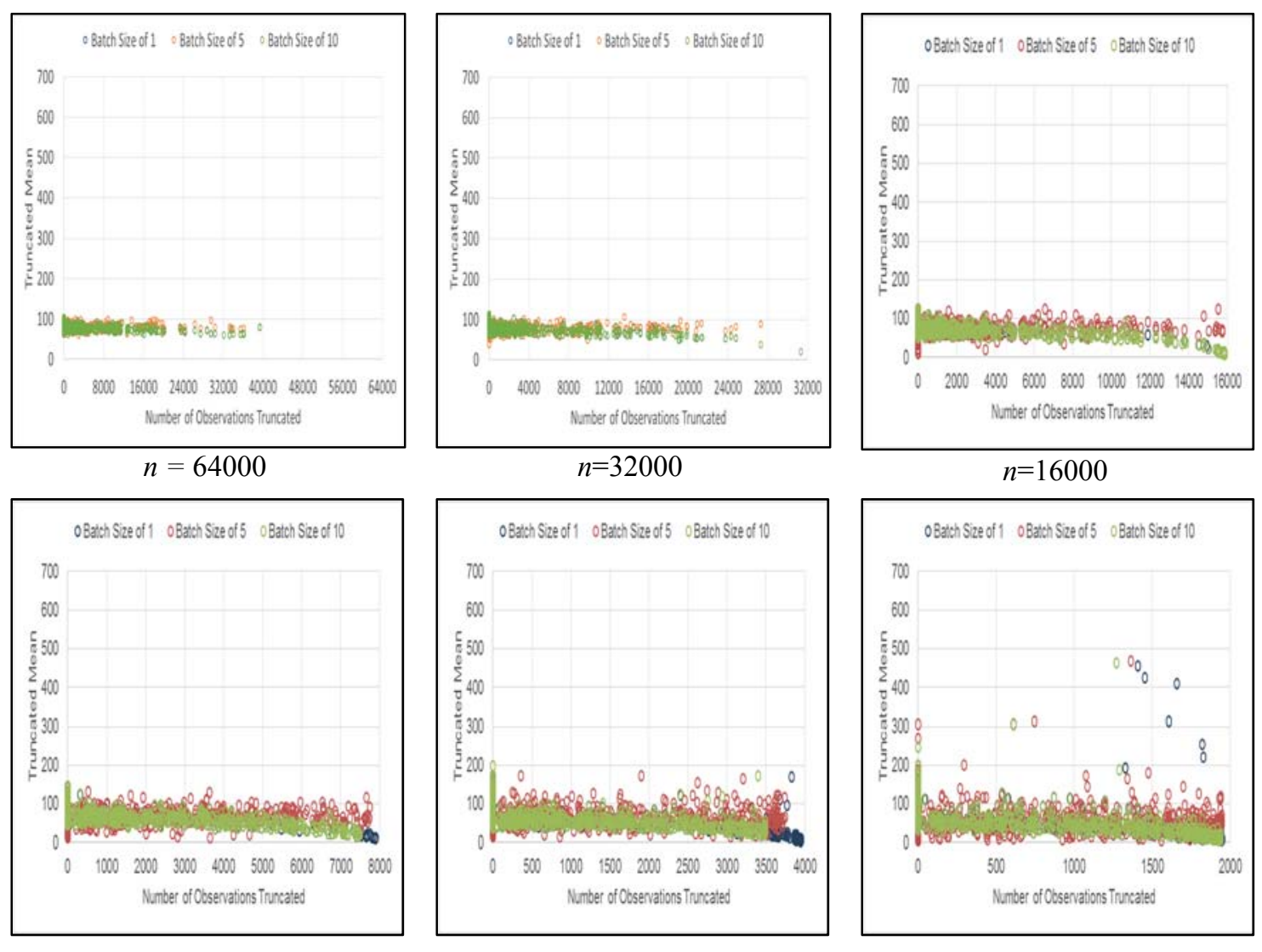

$n=4000$

$n=2000$

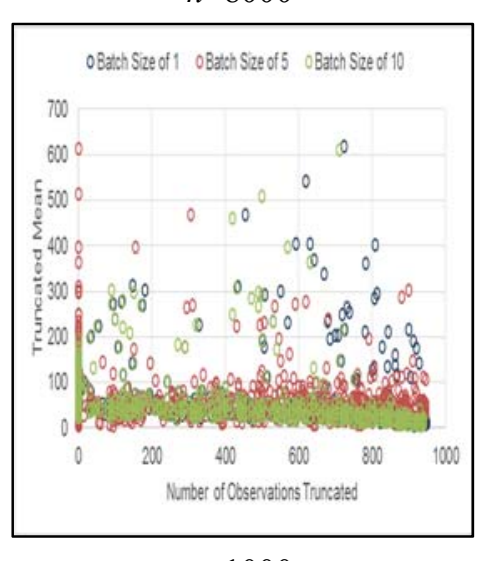

$$
n=1000
$$

Figure 7.23 Scatterplots of the truncated mean vs. the number of observations truncated for batch sizes $b=1,5$, and 10 for run length of $n=1000,2000,4000,8000,16000,32000$, and 64000 for Model 2 with traffic intensity of 0.9 
Table 7.8 Correlation between the truncated mean and the number of observations truncated for batch sizes $b=1,5$, and 10 for run length of $n=1000,2000,4000,8000,16000,32000$, and 64000 for Model 2 with traffic intensity of 0.9 .

\begin{tabular}{|l|l|l|l|}
\hline & $\begin{array}{l}\text { Trunc } \\
\text { Batch } \\
=1\end{array}$ & $\begin{array}{l}\text { Trunc } \\
\text { Batch } \\
=5\end{array}$ & $\begin{array}{l}\text { Trunc } \\
\text { Batch } \\
=10\end{array}$ \\
\hline $\begin{array}{l}\text { Mean } \\
\text { Batch } \\
=1\end{array}$ & $\mathbf{- 0 . 3 0 5 3}$ & -0.3019 & -0.2748 \\
\hline $\begin{array}{l}\text { Mean } \\
\text { Batch } \\
=5\end{array}$ & -0.3043 & $\mathbf{- 0 . 3 0 8 4}$ & -0.2809 \\
\hline $\begin{array}{l}\text { Mean } \\
\text { Batch } \\
=10\end{array}$ & -0.3003 & -0.3044 & $\mathbf{- 0 . 3 1 7 0}$ \\
\hline
\end{tabular}

\begin{tabular}{|l|l|l|l|}
\hline & $\begin{array}{l}\text { Trunc } \\
\text { Batch } \\
=1\end{array}$ & $\begin{array}{l}\text { Trunc } \\
\text { Batch } \\
=5\end{array}$ & $\begin{array}{l}\text { Trunc } \\
\text { Batch } \\
=10\end{array}$ \\
\hline $\begin{array}{l}\text { Mean } \\
\text { Batch } \\
=1\end{array}$ & $\mathbf{- 0 . 3 9 6 6}$ & -0.3687 & -0.3455 \\
\hline $\begin{array}{l}\text { Mean } \\
\text { Batch } \\
=5\end{array}$ & -0.4007 & $\mathbf{- 0 . 4 1 6 9}$ & -0.3976 \\
\hline $\begin{array}{l}\text { Mean } \\
\text { Batch } \\
=10\end{array}$ & -0.3854 & -0.4271 & $\mathbf{- 0 . 4 5 4 2}$ \\
\hline
\end{tabular}

\begin{tabular}{|l|l|l|l|}
\hline & $\begin{array}{l}\text { Trunc } \\
\text { Batch } \\
=1\end{array}$ & $\begin{array}{l}\text { Trunc } \\
\text { Batch } \\
=5\end{array}$ & $\begin{array}{l}\text { Trunc } \\
\text { Batch } \\
=10\end{array}$ \\
\hline $\begin{array}{l}\text { Mean } \\
\text { Batch } \\
=1\end{array}$ & $\mathbf{- 0 . 4 9 6 9}$ & -0.4433 & -0.4129 \\
\hline $\begin{array}{l}\text { Mean } \\
\text { Batch } \\
=5\end{array}$ & -0.4701 & $\mathbf{- 0 . 5 5 1 2}$ & -0.5107 \\
\hline $\begin{array}{l}\text { Mean } \\
\text { Batch } \\
=10\end{array}$ & -0.4570 & -0.5315 & $\mathbf{- 0 . 6 1 0 1}$ \\
\hline
\end{tabular}

$$
n=64000
$$

$$
n=32000
$$

\begin{tabular}{|l|l|l|l|}
\hline & $\begin{array}{l}\text { Trunc } \\
\text { Batch } \\
=1\end{array}$ & $\begin{array}{l}\text { Trunc } \\
\text { Batch } \\
=5\end{array}$ & $\begin{array}{l}\text { Trunc } \\
\text { Batch } \\
=10\end{array}$ \\
\hline $\begin{array}{l}\text { Mean } \\
\text { Batch } \\
=1\end{array}$ & $\mathbf{- 0 . 6 0 3 8}$ & -0.5261 & -0.4629 \\
\hline $\begin{array}{l}\text { Mean } \\
\text { Batch } \\
=5\end{array}$ & -0.5114 & $\mathbf{- 0 . 6 0 9 3}$ & -0.5238 \\
\hline $\begin{array}{l}\text { Mean } \\
\text { Batch } \\
=10\end{array}$ & -0.4389 & -0.5163 & $\mathbf{- 0 . 6 1 0 0}$ \\
\hline
\end{tabular}

$$
n=8000
$$

\begin{tabular}{|l|l|l|l|}
\hline & $\begin{array}{l}\text { Trunc } \\
\text { Batch } \\
=1\end{array}$ & $\begin{array}{l}\text { Trunc } \\
\text { Batch } \\
=5\end{array}$ & $\begin{array}{l}\text { Trunc } \\
\text { Batch } \\
=10\end{array}$ \\
\hline $\begin{array}{l}\text { Mean } \\
\text { Batch } \\
=1\end{array}$ & $\mathbf{- 0 . 6 1 8 9}$ & -0.5190 & -0.4686 \\
\hline $\begin{array}{l}\text { Mean } \\
\text { Batch } \\
=5\end{array}$ & -0.4419 & $\mathbf{- 0 . 5 7 1 4}$ & -0.5130 \\
\hline $\begin{array}{l}\text { Mean } \\
\text { Batch } \\
=10\end{array}$ & -0.2854 & -0.3969 & $\mathbf{- 0 . 5 2 9 5}$ \\
\hline
\end{tabular}

$$
n=4000
$$

\begin{tabular}{|l|l|l|l|}
\hline & $\begin{array}{l}\text { Trunc } \\
\text { Batch } \\
=1\end{array}$ & $\begin{array}{l}\text { Trunc } \\
\text { Batch } \\
=5\end{array}$ & $\begin{array}{l}\text { Trunc } \\
\text { Batch } \\
=10\end{array}$ \\
\hline $\begin{array}{l}\text { Mean } \\
\text { Batch } \\
=1\end{array}$ & $\mathbf{- 0 . 3 4 2 7}$ & -0.3867 & -0.4123 \\
\hline $\begin{array}{l}\text { Mean } \\
\text { Batch } \\
=5\end{array}$ & -0.3770 & $\mathbf{- 0 . 4 5 9 2}$ & -0.4741 \\
\hline $\begin{array}{l}\text { Mean } \\
\text { Batch } \\
=10\end{array}$ & -0.3774 & -0.4446 & $\mathbf{- 0 . 5 1 5 0}$ \\
\hline
\end{tabular}

$$
n=2000
$$

\begin{tabular}{|l|l|l|l|}
\hline & $\begin{array}{l}\text { Trunc } \\
\text { Batch } \\
=1\end{array}$ & $\begin{array}{l}\text { Trunc } \\
\text { Batch } \\
=5\end{array}$ & $\begin{array}{l}\text { Trunc } \\
\text { Batch } \\
=10\end{array}$ \\
\hline $\begin{array}{l}\text { Mean } \\
\text { Batch } \\
=1\end{array}$ & $\mathbf{- 0 . 0 8 2 0}$ & -0.2141 & -0.2591 \\
\hline $\begin{array}{l}\text { Mean } \\
\text { Batch } \\
=5\end{array}$ & -0.1102 & $\mathbf{- 0 . 2 2 7 0}$ & -0.2725 \\
\hline $\begin{array}{l}\text { Mean } \\
\text { Batch } \\
=10\end{array}$ & -0.1215 & -0.2397 & $\mathbf{- 0 . 2 8 0 5}$ \\
\hline
\end{tabular}

$$
n=1000
$$


- Batch Size of 1 - Batch Size of 5 - Batch Size of 10

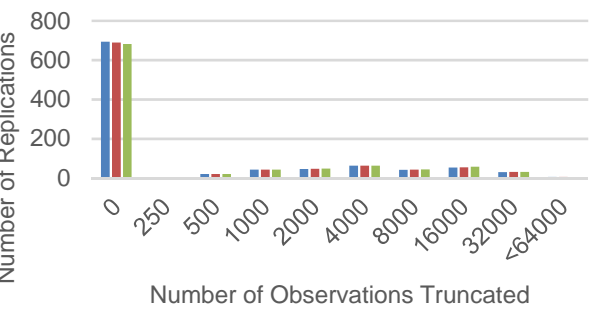

$$
n=64000
$$

Batch Size of 1 Batch Size of 5 Batch Size of 10

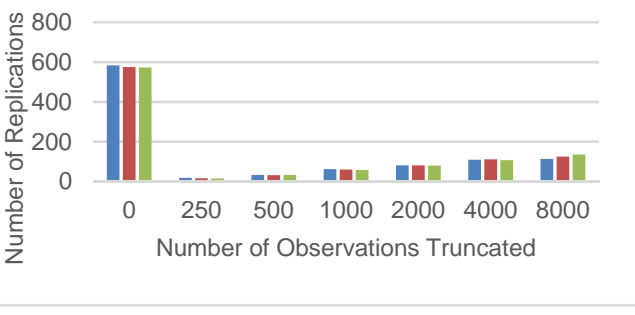

$n=8000$

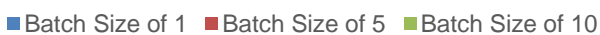

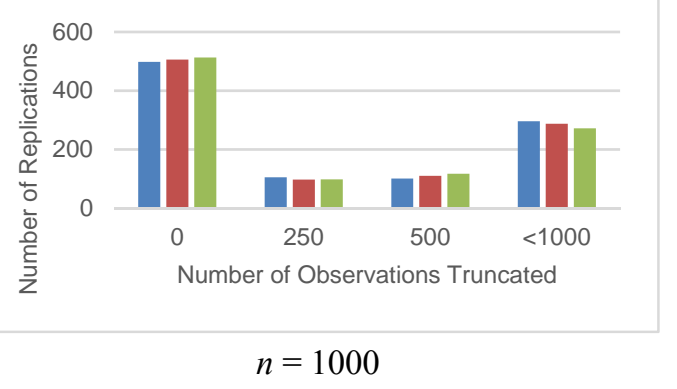

- Batch Size of 1 - Batch Size of 5 m Batch Size of 10

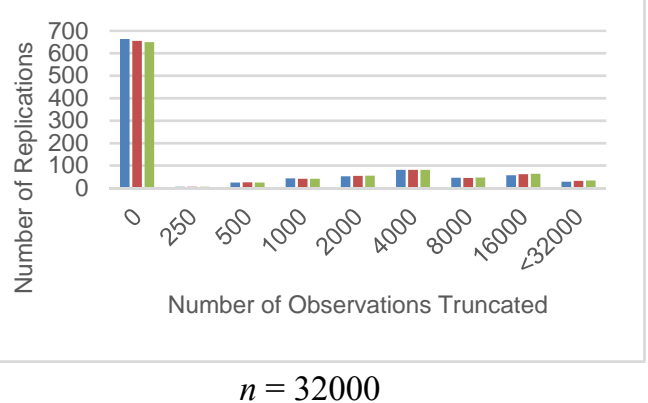

match Size of 1 Batch Size of 5 Batch Size of 10

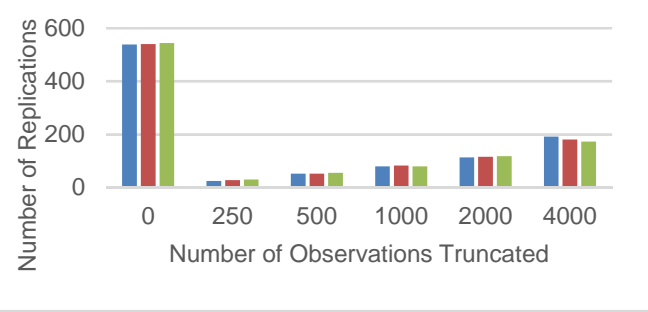

$n=4000$
- Batch Size of 1 - Batch Size of 5 match Size of 10

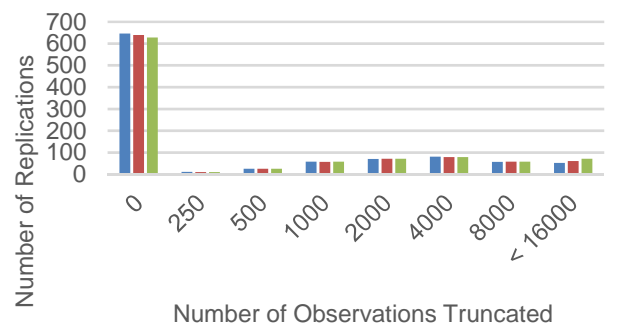

$$
n=16000
$$

-Batch Size of 1 Batch Size of 5

Batch Size of 10

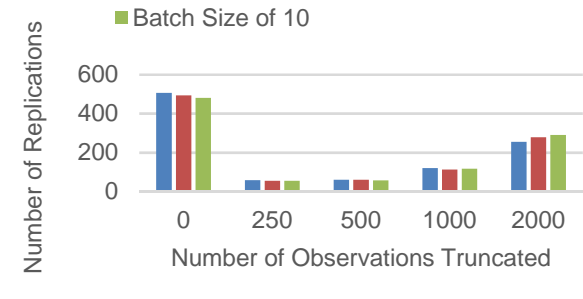

$n=2000$

Figure 7.24 Frequency distribution of the number of observations truncated as a function of batch sizes $b=1,5$, and 10 for run length of $n=1000,2000,4000,8000,16000,32000$, and 64000 for Model 2 with traffic intensity of 0.9 
7.2.2.2 Results for Model 2 with traffic intensity of $\mathbf{0 . 8 0}$

$$
n=16000 \text {, traffic intensity of } 0.8
$$

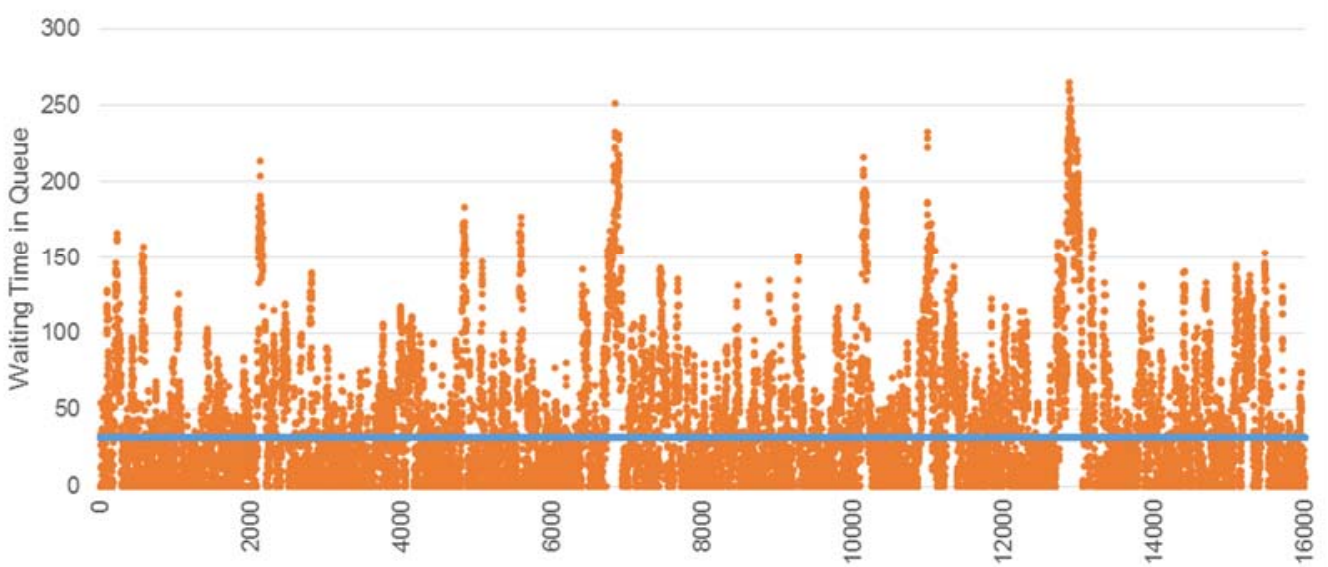

Figure 7.25 Example of M/M/1 with traffic intensity of $0.8(n=16000$, Blue line: $E(X))$
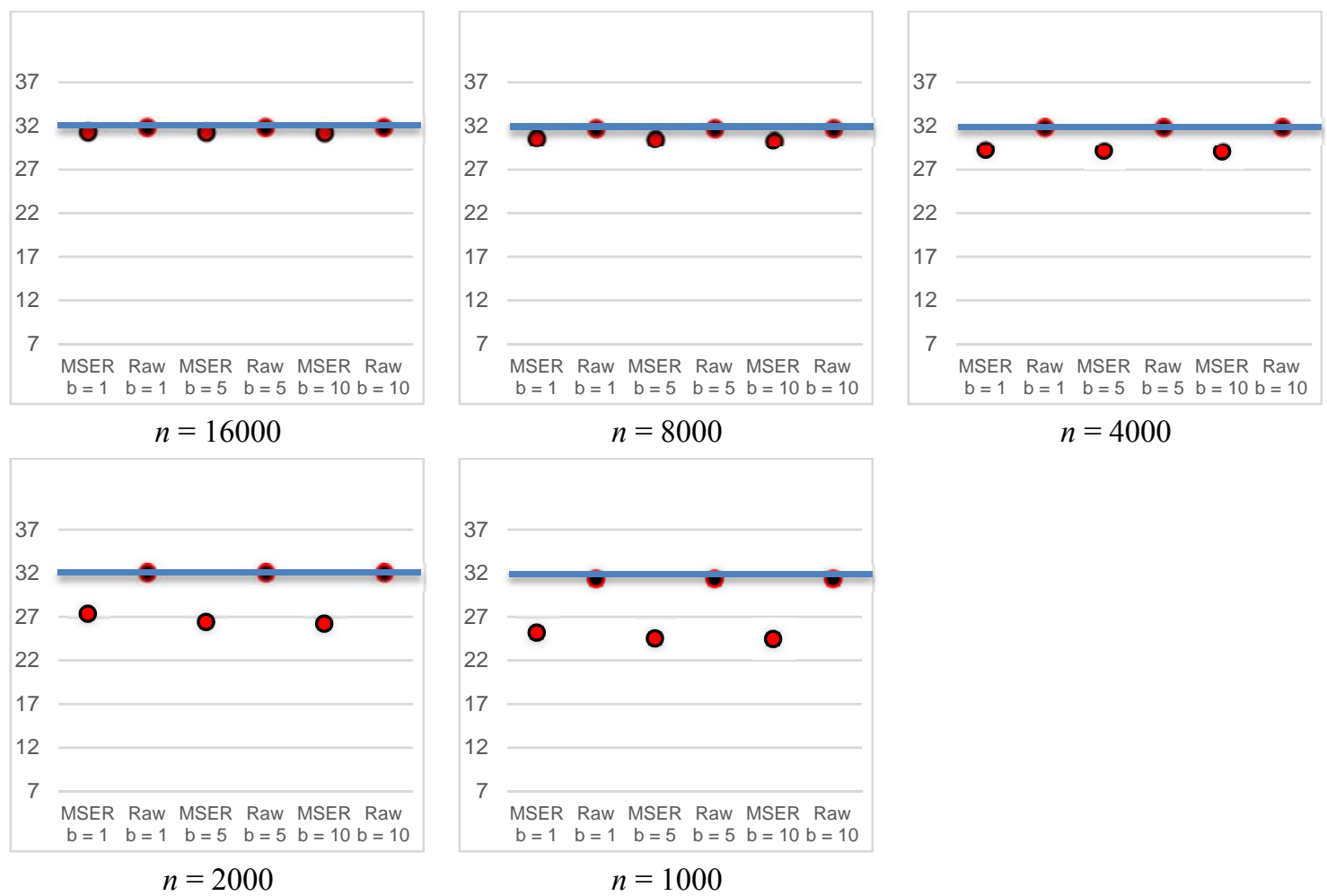

Figure $7.2695 \%$ confidence intervals of the mean for the truncated mean output as a function of batch size $(b=1,5$, and 10) and run length $(n=1000,2000,4000,8000$, and 16,000) for Model 2 with traffic intensity of 0.80 (Theoretical mean of 32, Blue line) 

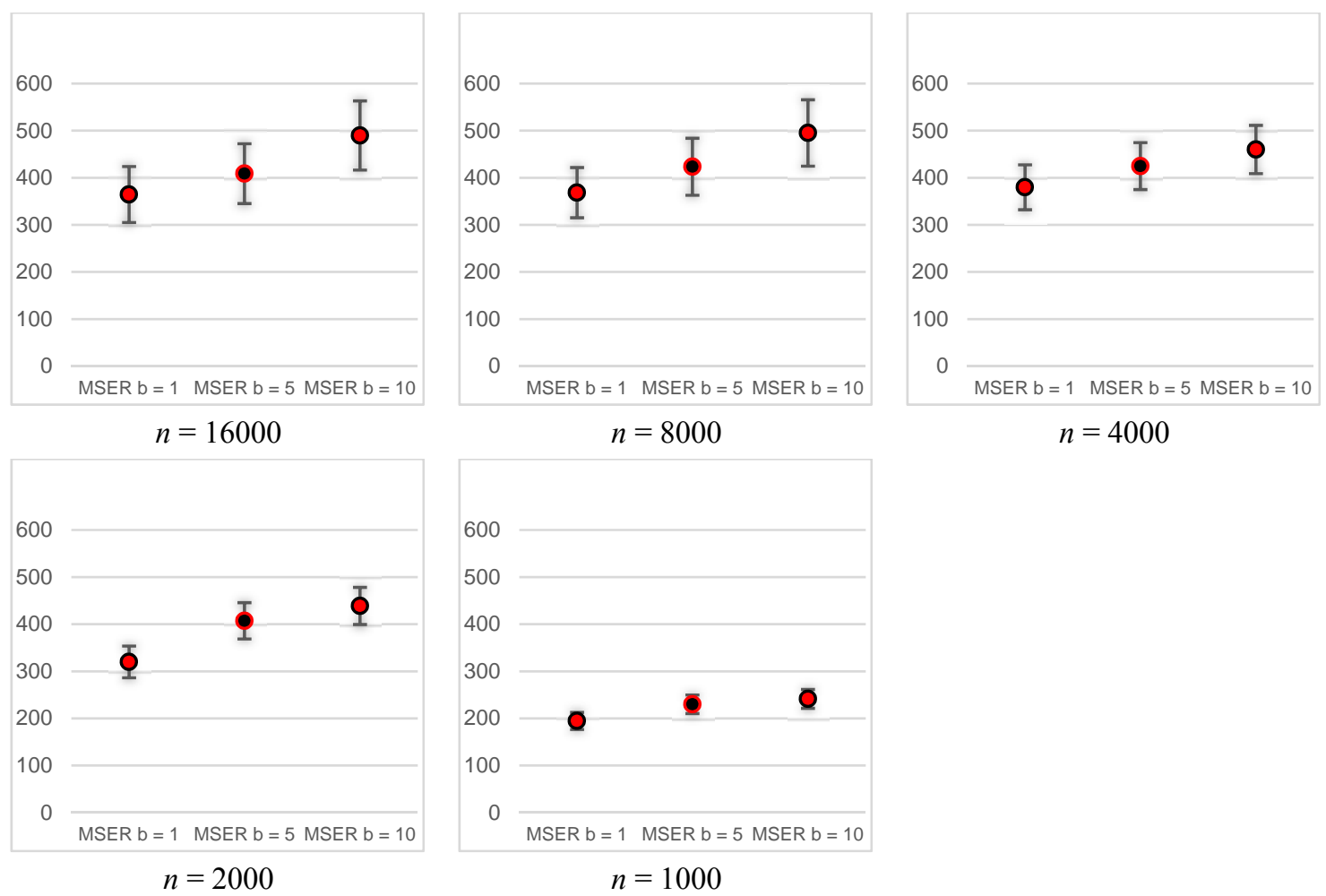

Figure $7.2795 \%$ confidence intervals for the mean number of observations truncated as a function of batch size $(b=1,5$, and 10$)$ and run length $(n=1000,2000,4000,8000$, and 16,000) for Model 2 with traffic intensity of 0.80 


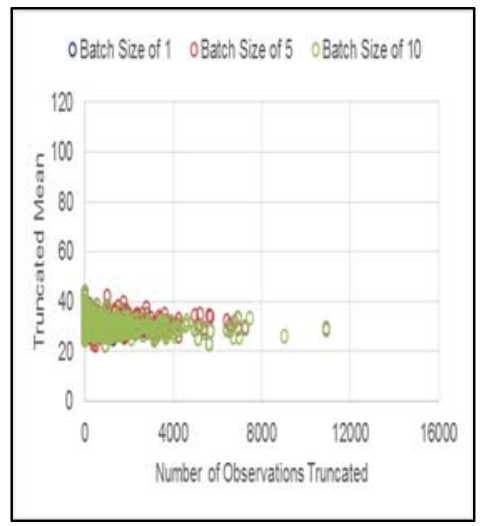

$n=16000$

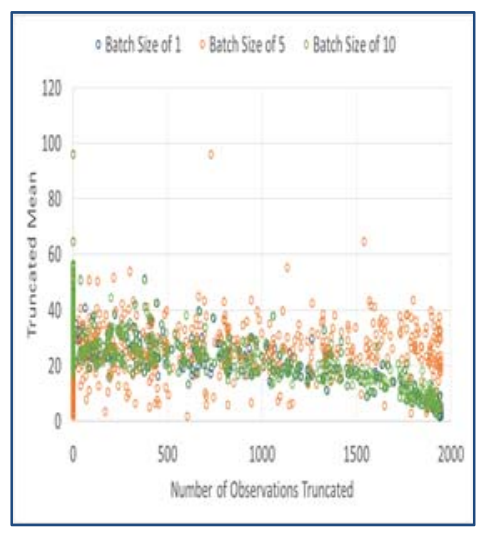

$n=2000$

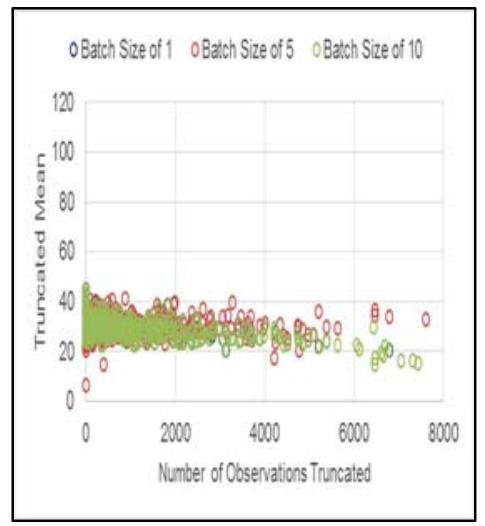

$n=8000$

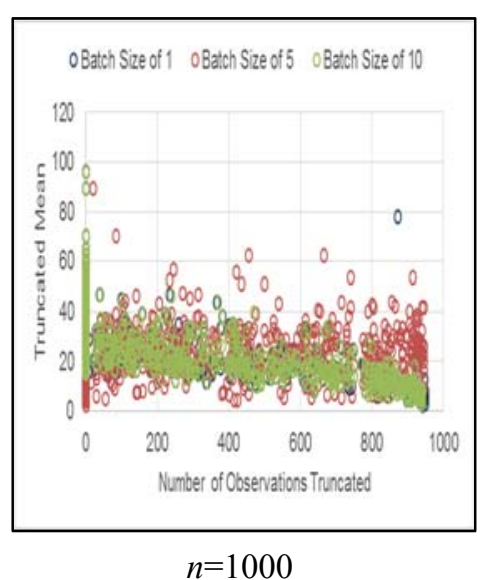

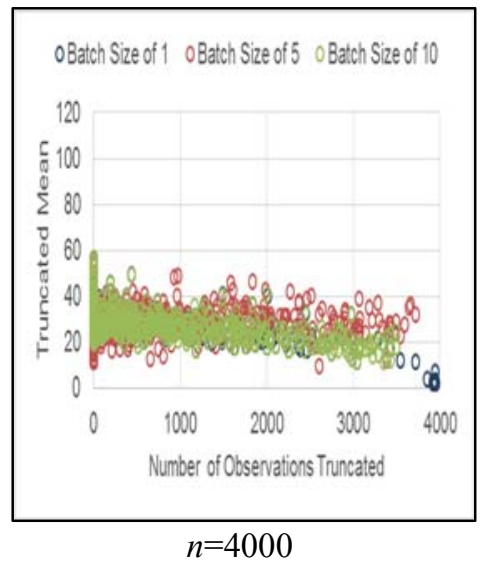

$n=4000$

Figure 7.28 Scatterplots of the truncated mean vs. the number of observations truncated for batch sizes $b=1,5$, and 10 for run length of $n=1000,2000,4000,8000$, and 16,000 Model 2 with traffic intensity of 0.80 
Table 7.9 Correlation between the truncated mean and the number of observations truncated for batch sizes $b=1,5$, and 10 for run length of $n=1000,2000,4000,8000$, and 16,000 for Model 2 with traffic intensity of 0.80

\begin{tabular}{|l|l|l|l|}
\hline & $\begin{array}{l}\text { Trunc } \\
\text { Batch } \\
=1\end{array}$ & $\begin{array}{l}\text { Trunc } \\
\text { Batch } \\
=5\end{array}$ & $\begin{array}{l}\text { Trunc } \\
\text { Batch } \\
=10\end{array}$ \\
\hline $\begin{array}{l}\text { Mean } \\
\text { Batch }= \\
1\end{array}$ & $\mathbf{- 0 . 2 3 0 9}$ & -0.2198 & -0.1629 \\
\hline $\begin{array}{l}\text { Mean } \\
\text { Batch }= \\
5\end{array}$ & -0.2305 & $\mathbf{- 0 . 2 5 0 9}$ & -0.1892 \\
\hline $\begin{array}{l}\text { Mean } \\
\text { Batch }= \\
10\end{array}$ & -0.2270 & -0.2467 & $\mathbf{- 0 . 2 7 1 8}$ \\
\hline
\end{tabular}

$n=16000$

\begin{tabular}{|l|l|l|l|}
\hline & $\begin{array}{l}\text { Trunc } \\
\text { Batch } \\
=1\end{array}$ & $\begin{array}{l}\text { Trunc } \\
\text { Batch =5 }\end{array}$ & $\begin{array}{l}\text { Trunc } \\
\text { Batch } \\
=10\end{array}$ \\
\hline $\begin{array}{l}\text { Mean } \\
\text { Batch } \\
=1\end{array}$ & $\mathbf{- 0 . 3 5 7 5}$ & -0.2954 & -0.1696 \\
\hline $\begin{array}{l}\text { Mean } \\
\text { Batch } \\
=5\end{array}$ & -0.3339 & $\mathbf{- 0 . 4 3 1 0}$ & -0.2472 \\
\hline $\begin{array}{l}\text { Mean } \\
\text { Batch } \\
=10\end{array}$ & -0.3162 & -0.3654 & $\mathbf{- 0 . 4 7 4 1}$ \\
\hline
\end{tabular}

\begin{tabular}{|l|l|l|l|}
\hline & $\begin{array}{l}\text { Trunc } \\
\text { Batch } \\
=1\end{array}$ & $\begin{array}{l}\text { Trunc } \\
\text { Batch } \\
=5\end{array}$ & $\begin{array}{l}\text { Trunc } \\
\text { Batch } \\
=10\end{array}$ \\
\hline $\begin{array}{l}\text { Mean } \\
\text { Batch } \\
=1\end{array}$ & $\mathbf{- 0 . 5 7 1 2}$ & -0.3760 & -0.3494 \\
\hline $\begin{array}{l}\text { Mean } \\
\text { Batch } \\
=5\end{array}$ & -0.4004 & $\mathbf{- 0 . 5 5 9 6}$ & -0.4806 \\
\hline $\begin{array}{l}\text { Mean } \\
\text { Batch } \\
=10\end{array}$ & -0.3643 & -0.4781 & $\mathbf{- 0 . 5 6 2 7}$ \\
\hline
\end{tabular}

\begin{tabular}{|l|l|l|l|}
\hline & $\begin{array}{l}\text { Trunc } \\
\text { Batch } \\
=1\end{array}$ & $\begin{array}{l}\text { Trunc } \\
\text { Batch } \\
=5\end{array}$ & $\begin{array}{l}\text { Trunc } \\
\text { Batch } \\
=10\end{array}$ \\
\hline $\begin{array}{l}\text { Mean } \\
\begin{array}{l}\text { Batch } \\
=1\end{array}\end{array}$ & $\mathbf{- 0 . 6 4 5 0}$ & -0.0322 & -0.0362 \\
\hline $\begin{array}{l}\text { Mean } \\
\text { Batch } \\
=5\end{array}$ & 0.0895 & $\mathbf{- 0 . 7 0 7 2}$ & -0.6257 \\
\hline $\begin{array}{l}\text { Mean } \\
\text { Batch } \\
=10\end{array}$ & 0.1010 & -0.6245 & $\mathbf{- 0 . 7 1 4 7}$ \\
\hline
\end{tabular}

$n=2000$

\begin{tabular}{|l|l|l|l|}
\hline & $\begin{array}{l}\text { Trunc } \\
\text { Batch } \\
=1\end{array}$ & $\begin{array}{l}\text { Trunc } \\
\text { Batch } \\
=5\end{array}$ & $\begin{array}{l}\text { Trunc } \\
\text { Batch } \\
=10\end{array}$ \\
\hline $\begin{array}{l}\text { Mean } \\
\text { Batch }= \\
1\end{array}$ & $\mathbf{- 0 . 5 8 4 0}$ & -0.5342 & -0.5021 \\
\hline $\begin{array}{l}\text { Mean } \\
\text { Batch }= \\
5\end{array}$ & -0.5326 & $\mathbf{- 0 . 6 4 7 1}$ & -0.6008 \\
\hline $\begin{array}{l}\text { Mean } \\
\text { Batch }= \\
10\end{array}$ & -0.4800 & -0.5831 & $\mathbf{- 0 . 6 4 8 6}$ \\
\hline
\end{tabular}

$n=4000$

$n=1000$ 
-Batch Size of 1 - Batch Size of 5 Batch Size of 10

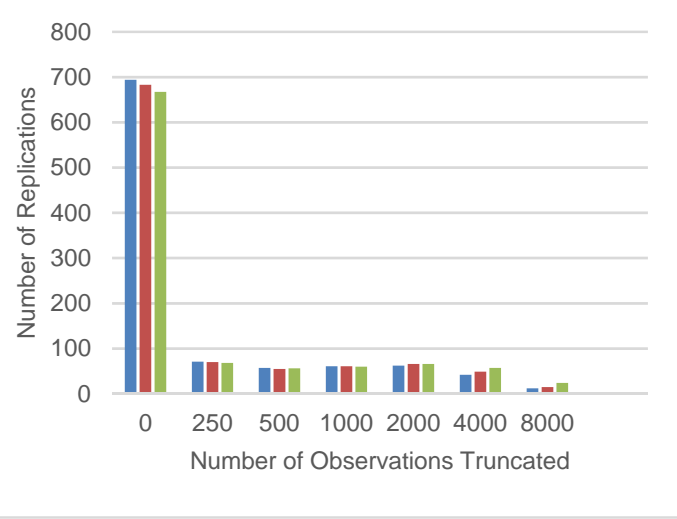

$$
n=16000
$$

-Batch Size of 1 -Batch Size of 5 Batch Size of 10

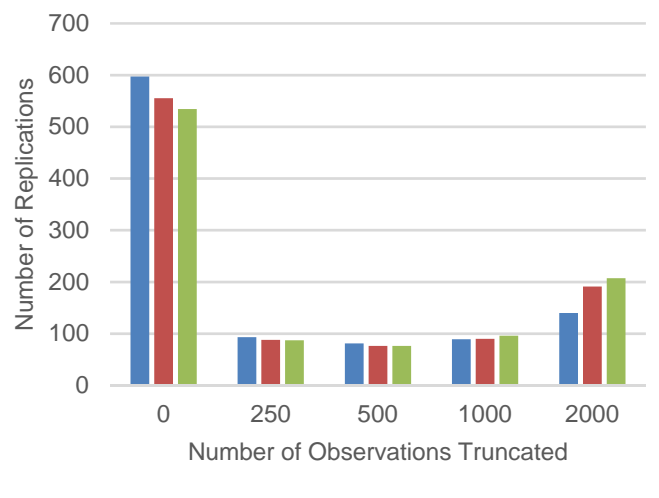

$n=2000$
- Batch Size of 1 - Batch Size of 5 Batch Size of 10

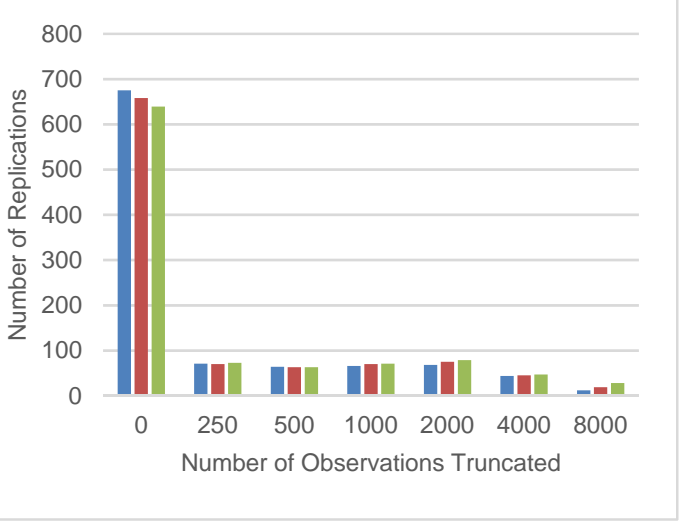

$n=8000$

-Batch Size of 1 -Batch Size of 5 Batch Size of 10

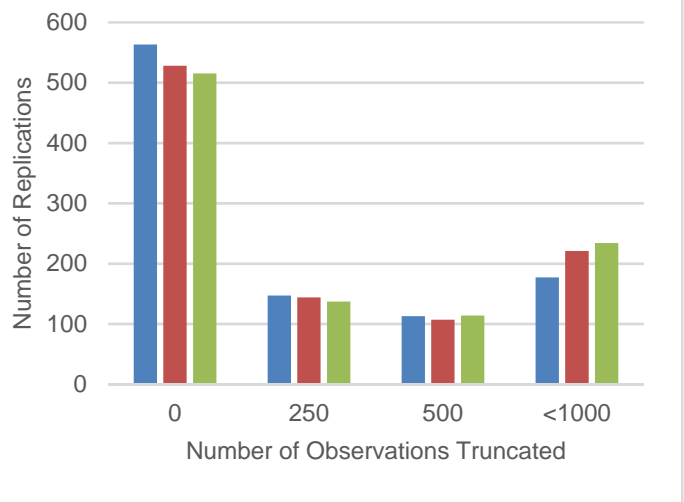

$n=1000$
- Batch Size of 1 - Batch Size of 5 - Batch Size of 10

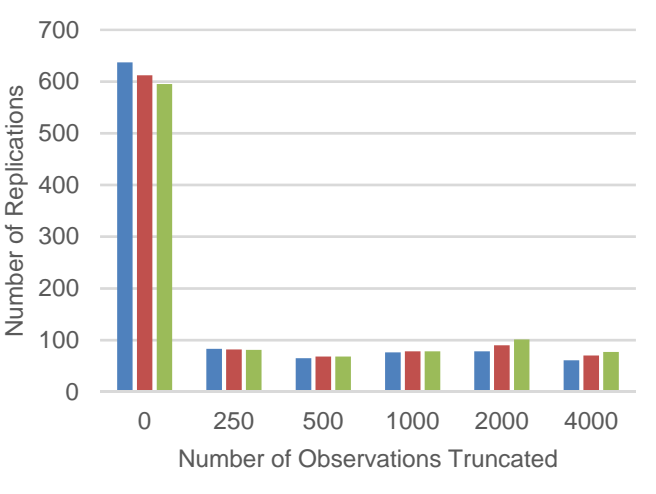

$n=4000$

Figure 7.29 Frequency distribution of the number of observations truncated as a function of batch sizes $b=1,5$, and 10 for run length of $n=1000,2000,4000,8000$, and 16,000 for Model 2 with traffic intensity of 0.80 
7.2.2.3 Results for Model 2 with traffic intensity of $\mathbf{0 . 7 0}$

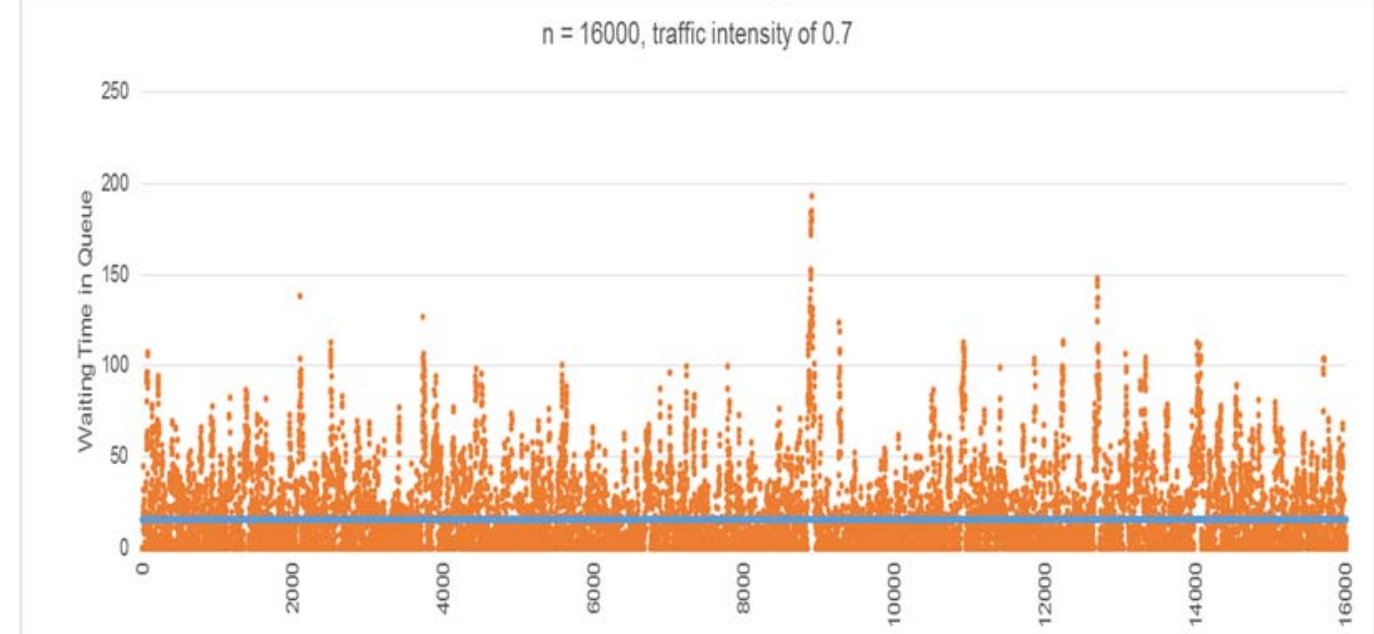

Figure 7.30 Example of $M / M / 1$ with traffic intensity of $0.70(n=16000$, Blue line: $E(X))$
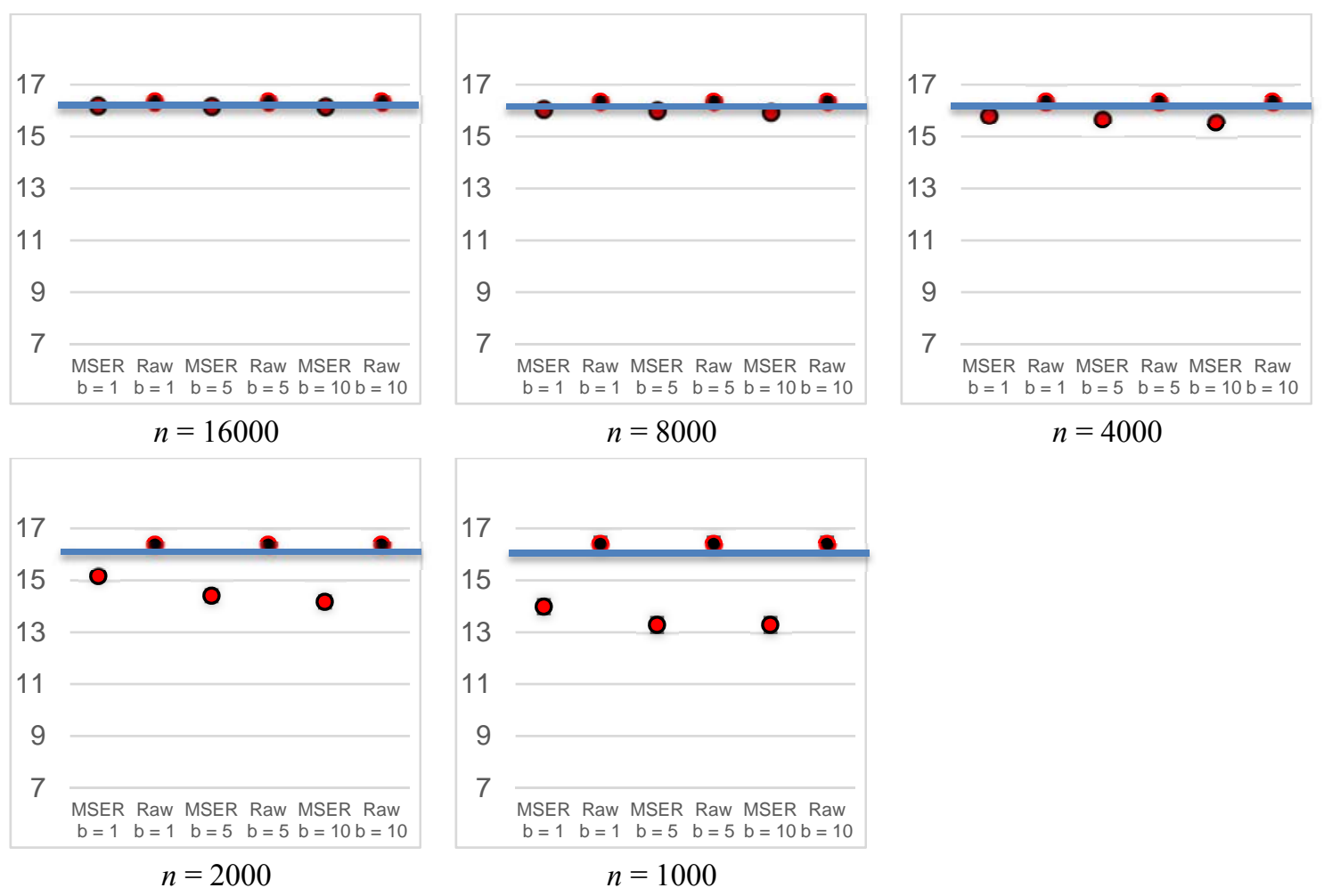

Figure $7.3195 \%$ confidence intervals of the mean for the truncated mean output as a function of batch size $(b=1,5$, and 10) and run length $(n=1000,2000,4000,8000$, and 16,000 ) for Model 2 with traffic intensity of 0.70 (Theoretical mean of 16.33, Blue line) 

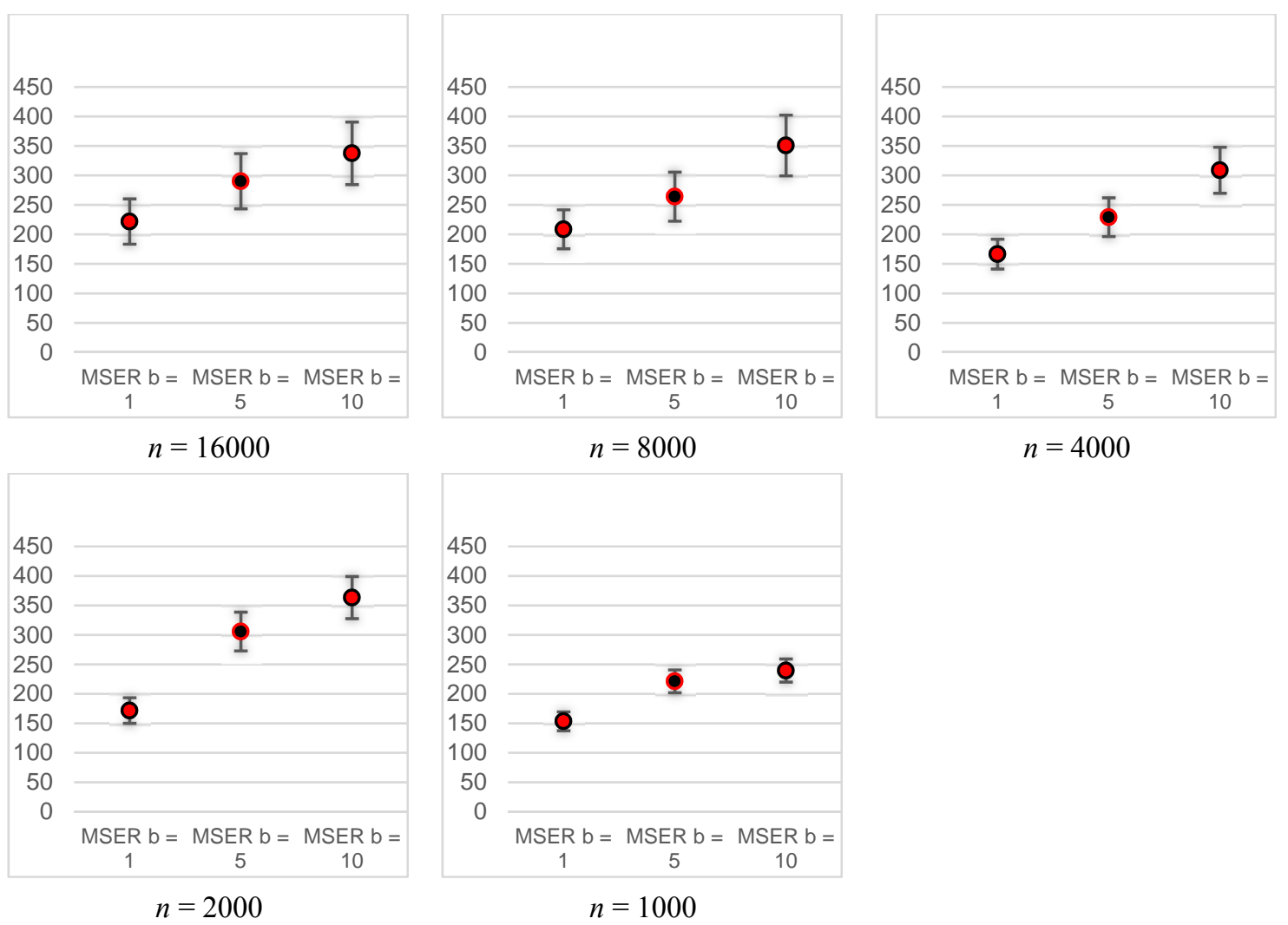

Figure $7.3295 \%$ confidence intervals for the mean number of observations truncated as a function of batch size $(b=1,5$, and 10$)$ and run length $(n=1000,2000,4000,8000$, and 16,000) for Model 2 with traffic intensity of 0.70 


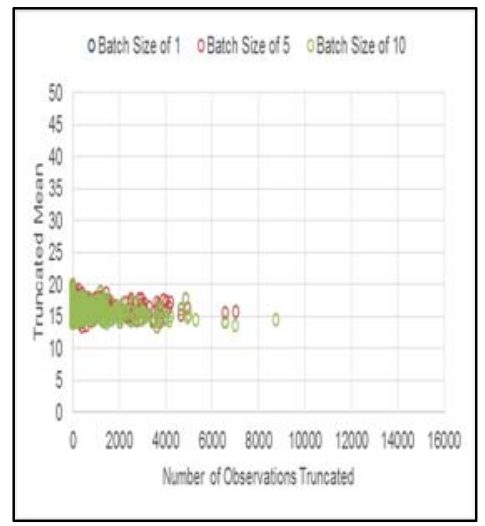

$n=16000$

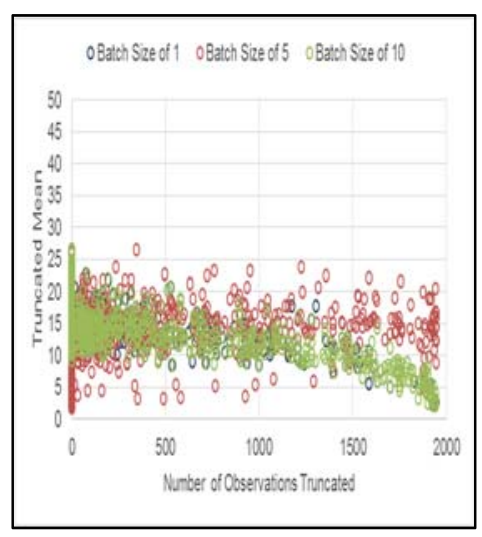

$n=2000$

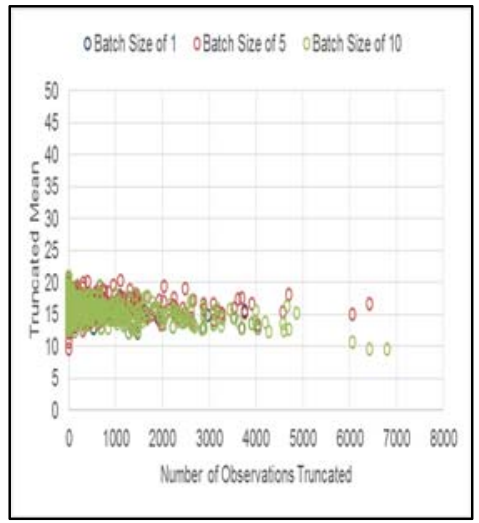

$n=8000$

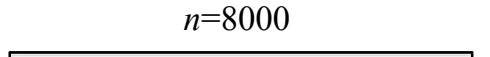

OBatch Size of 1 OBatch Size of 5 OBath Size of 10

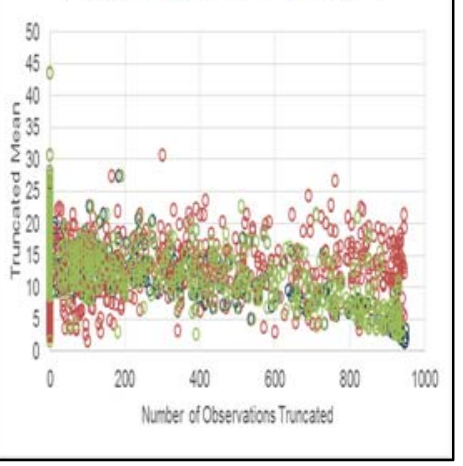

$n=1000$

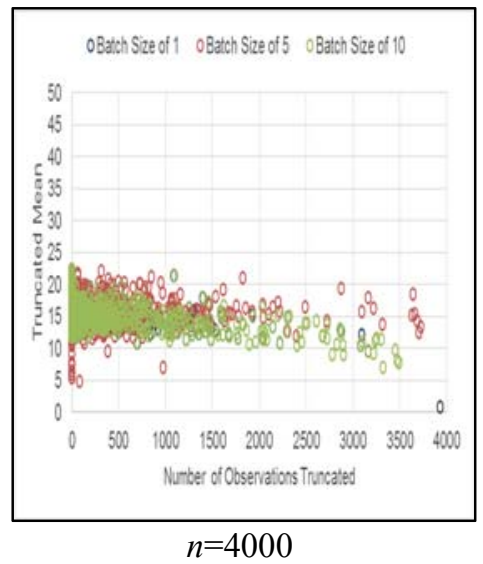

Figure 7.33 Scatterplots of the truncated mean vs. the number of observations truncated for batch sizes $b=1,5$, and 10 for run length of $n=1000,2000,4000,8000$, and 16,000 Model 2 with traffic intensity of 0.7 
Table 7.10 Correlation between the truncated mean and the number of observations truncated for batch sizes $b=1,5$, and 10 for run length of $n=1000,2000,4000,8000$, and 16,000 for Model 2 with traffic intensity of 0.70

\begin{tabular}{|l|l|l|l|}
\hline & $\begin{array}{l}\text { Trunc } \\
\text { Batch } \\
=1\end{array}$ & $\begin{array}{l}\text { Trunc } \\
\text { Batch } \\
=5\end{array}$ & $\begin{array}{l}\text { Trunc } \\
\text { Batch } \\
=10\end{array}$ \\
\hline $\begin{array}{l}\text { Mean } \\
\text { Batch } \\
=1\end{array}$ & -0.2111 & -0.1440 & -0.1260 \\
\hline $\begin{array}{l}\text { Mean } \\
\text { Batch } \\
=5\end{array}$ & -0.2043 & -0.2309 & -0.2020 \\
\hline $\begin{array}{l}\text { Mean } \\
\text { Batch } \\
=10\end{array}$ & -0.2032 & -0.2288 & -0.2547 \\
\hline
\end{tabular}

\begin{tabular}{|l|l|l|l|}
\hline & $\begin{array}{l}\text { Trunc } \\
\text { Batch } \\
=1\end{array}$ & $\begin{array}{l}\text { Trunc } \\
\text { Batch } \\
=5\end{array}$ & $\begin{array}{l}\text { Trunc } \\
\text { Batch } \\
=10\end{array}$ \\
\hline $\begin{array}{l}\text { Mean } \\
\text { Batch } \\
=1\end{array}$ & -0.2798 & -0.1877 & -0.0755 \\
\hline $\begin{array}{l}\text { Mean } \\
\text { Batch } \\
=5\end{array}$ & -0.2774 & -0.3459 & -0.2021 \\
\hline $\begin{array}{l}\text { Mean } \\
\text { Batch } \\
=10\end{array}$ & -0.2628 & -0.3292 & -0.3816 \\
\hline
\end{tabular}

\begin{tabular}{|l|l|l|l|}
\hline & $\begin{array}{l}\text { Trunc } \\
\text { Batch } \\
=1\end{array}$ & $\begin{array}{l}\text { Trunc } \\
\text { Batch } \\
=5\end{array}$ & $\begin{array}{l}\text { Trunc } \\
\text { Batch } \\
=10\end{array}$ \\
\hline $\begin{array}{l}\text { Mean } \\
\text { Batch } \\
=1\end{array}$ & -0.3720 & -0.1779 & -0.1079 \\
\hline $\begin{array}{l}\text { Mean } \\
\text { Batch } \\
=5\end{array}$ & -0.3015 & -0.4755 & -0.2573 \\
\hline $\begin{array}{l}\text { Mean } \\
\text { Batch } \\
=10\end{array}$ & -0.2865 & -0.3195 & -0.4833 \\
\hline
\end{tabular}

$$
n=16000
$$

$$
n=8000
$$

$n=4000$

\begin{tabular}{|l|l|l|l|}
\hline & $\begin{array}{l}\text { Trunc } \\
\text { Batch } \\
=1\end{array}$ & $\begin{array}{l}\text { Trunc } \\
\text { Batch } \\
=5\end{array}$ & $\begin{array}{l}\text { Trunc } \\
\text { Batch } \\
=10\end{array}$ \\
\hline $\begin{array}{l}\text { Mean } \\
\text { Batch } \\
=1\end{array}$ & -0.5000 & -0.2143 & -0.1666 \\
\hline $\begin{array}{l}\text { Mean } \\
\text { Batch } \\
=5\end{array}$ & -0.3846 & -0.7016 & -0.5412 \\
\hline $\begin{array}{l}\text { Mean } \\
\text { Batch } \\
=10\end{array}$ & -0.3682 & -0.5999 & -0.7216 \\
\hline
\end{tabular}

$n=2000$

\begin{tabular}{|l|l|l|l|}
\hline & $\begin{array}{l}\text { Trunc } \\
\text { Batch } \\
=1\end{array}$ & $\begin{array}{l}\text { Trunc } \\
\text { Batch } \\
=5\end{array}$ & $\begin{array}{l}\text { Trunc } \\
\text { Batch } \\
=10\end{array}$ \\
\hline $\begin{array}{l}\text { Mean } \\
\text { Batch } \\
=1\end{array}$ & -0.6094 & -0.4215 & -0.4041 \\
\hline $\begin{array}{l}\text { Mean } \\
\text { Batch } \\
=5\end{array}$ & -0.4924 & -0.6921 & -0.6256 \\
\hline $\begin{array}{l}\text { Mean } \\
\text { Batch } \\
=10\end{array}$ & -0.4924 & -0.6921 & -0.6256 \\
\hline
\end{tabular}

$n=1000$ 
- Batch Size of 1 - Batch Size of 5 Batch Size of 10

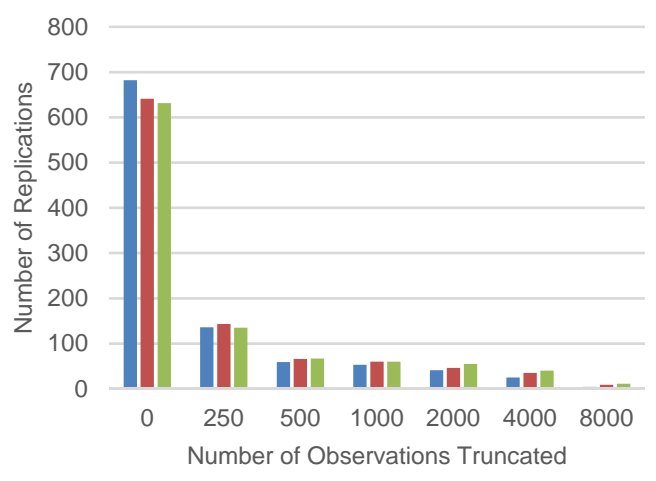

$n=16000$

-Batch Size of 1 Batch Size of 5 Batch Size of 10

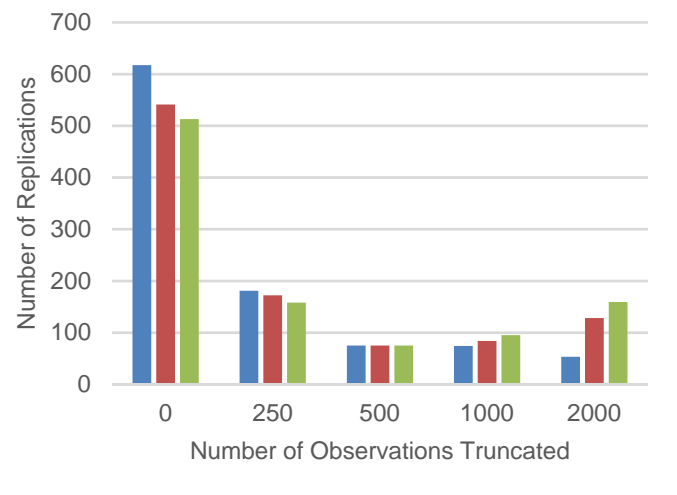

$n=2000$
- Batch Size of 1 - Batch Size of 5 - Batch Size of 10

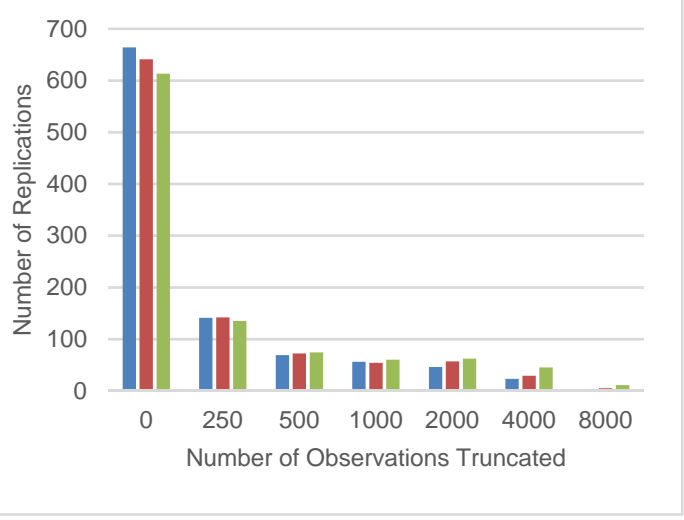

$n=8000$

- Batch Size of 1 Batch Size of 5 Batch Size of 10

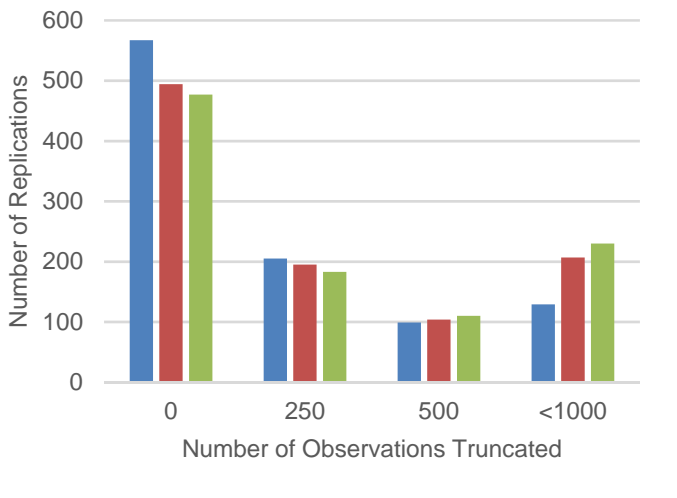

- Batch Size of 1 - Batch Size of 5 Batch Size of 10

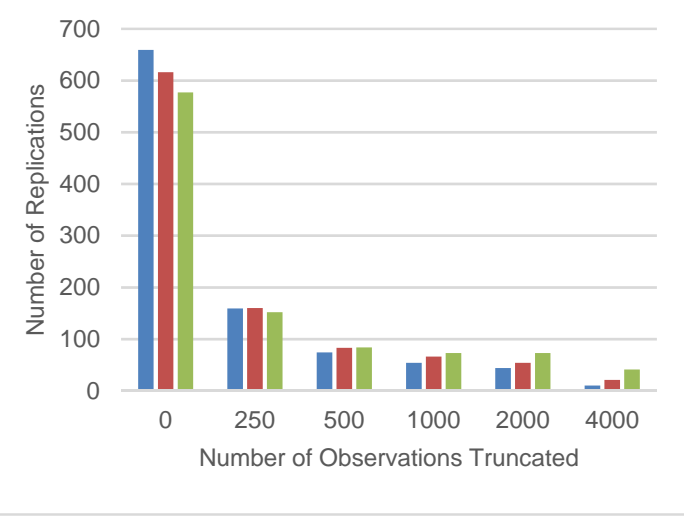

$n=4000$

$n=1000$

Figure 7.34 Frequency distribution of the number of observations truncated as a function of batch sizes $b=1,5$, and 10 for run length of $n=1000,2000,4000$, 8000 , and 16,000 for Model 2 with traffic intensity of 0.70 
7.2.2.4 Results for Model 2 with traffic intensity of 0.60

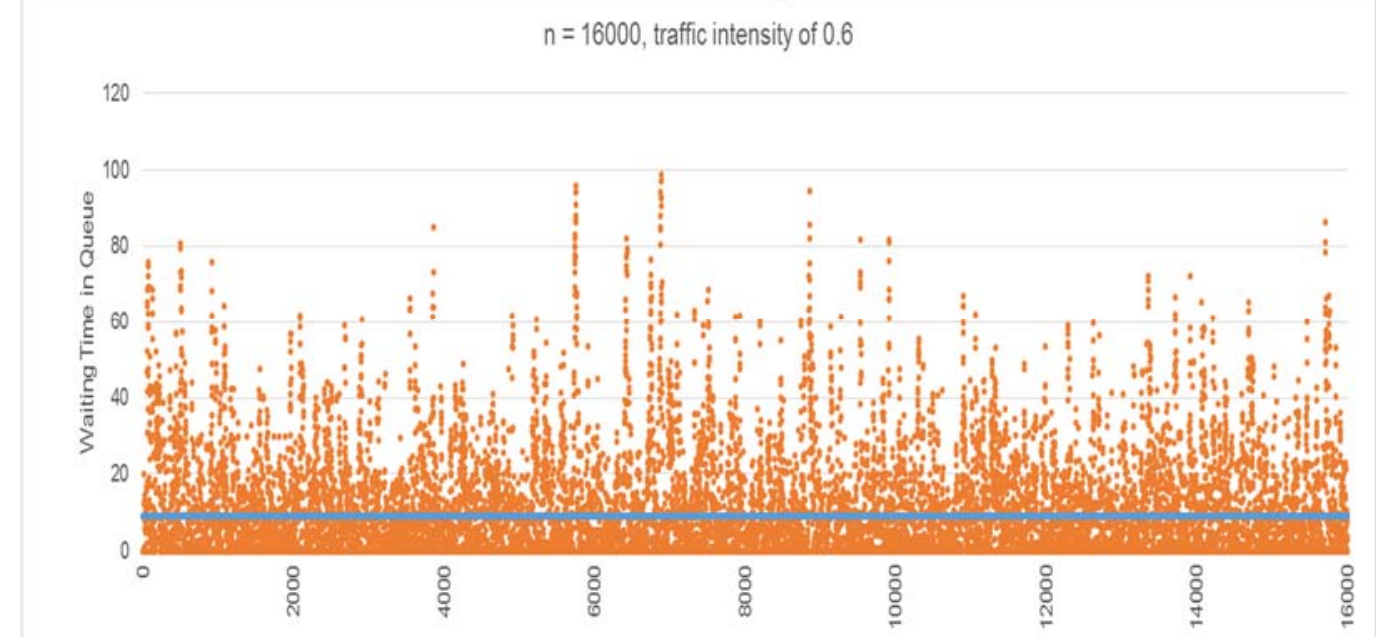

Figure 7.35 Example of $M / M / 1$ with traffic intensity of $0.60(n=16000$, Blue line: $E(X))$
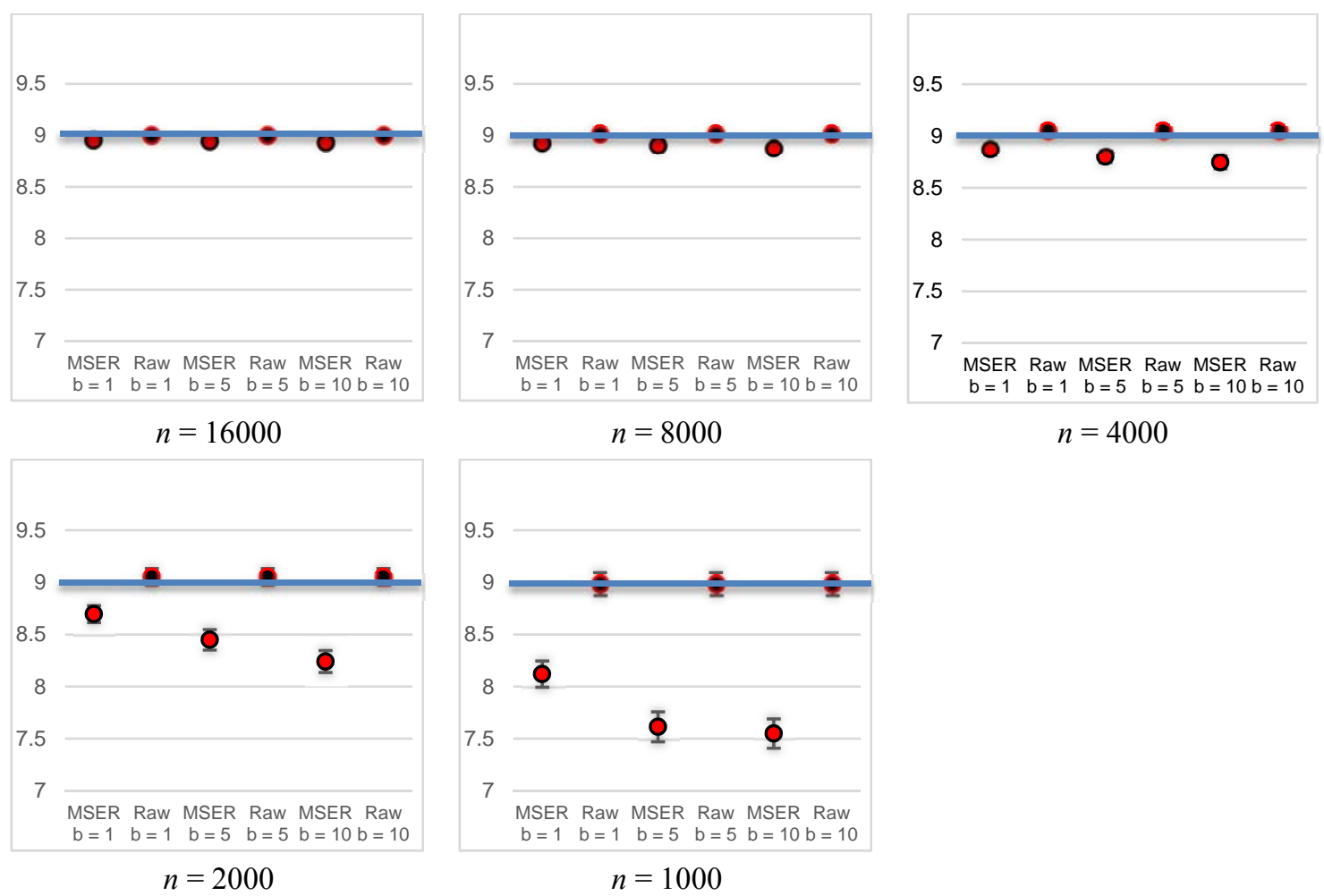

Figure.7.36 95\% confidence intervals of the mean for the truncated mean output as a function of batch size $(b=1,5$, and 10) and run length $(n=1000,2000,4000,8000$, and 16,000) for Model 2 with traffic intensity of 0.60 (Theoretical mean of 9, Blue line) 

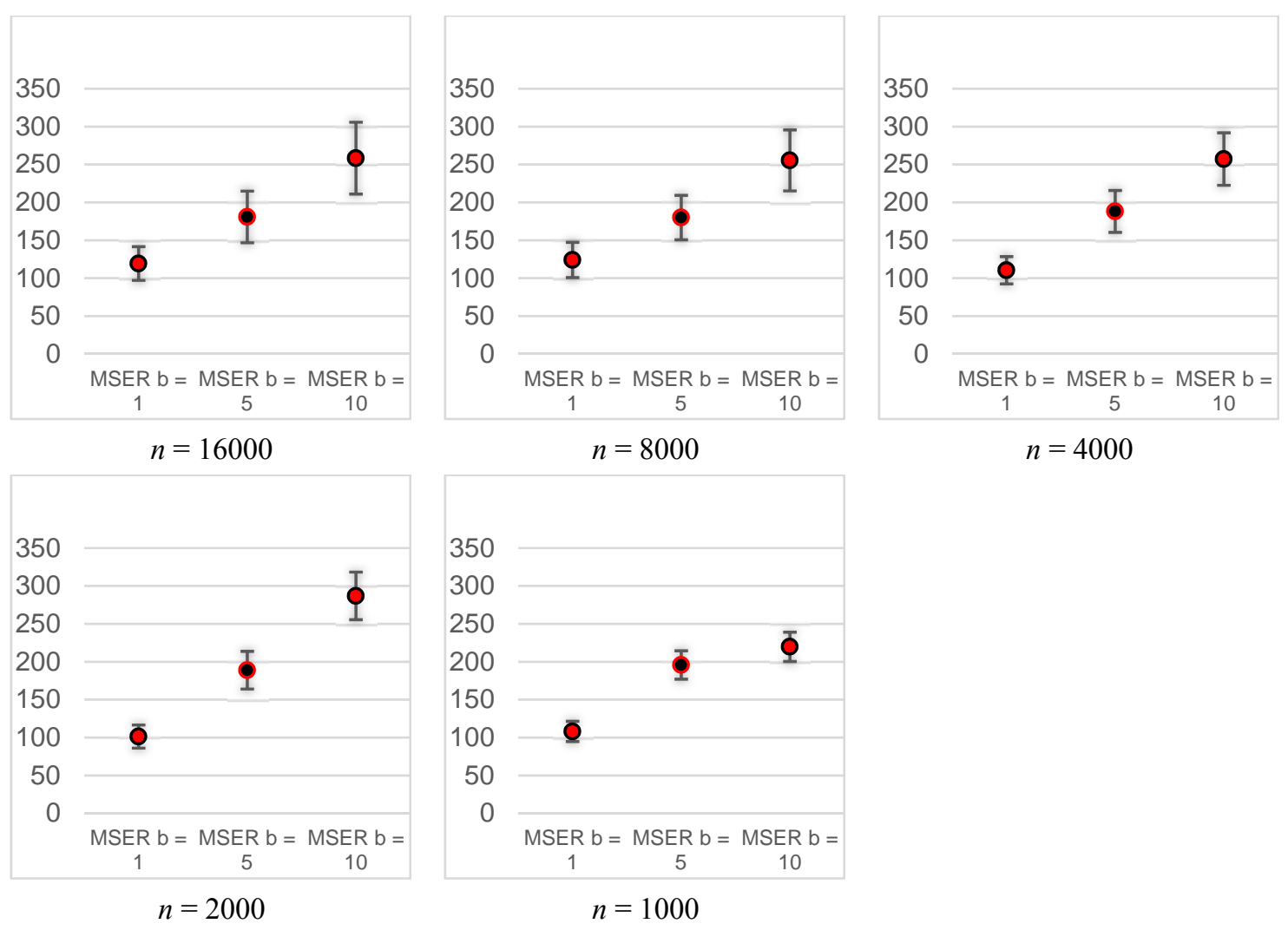

Figure $7.3795 \%$ confidence intervals for the mean number of observations truncated as a function of batch size $(b=1,5$, and 10) and run length $(n=1000,2000,4000,8000$, and 16,000) for Model 2 with traffic intensity of 0.60 


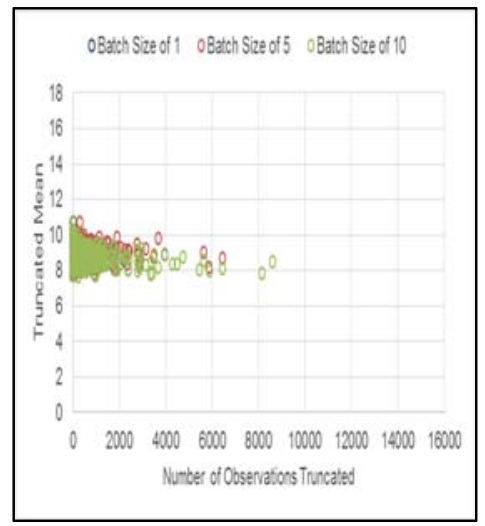

$n=16000$

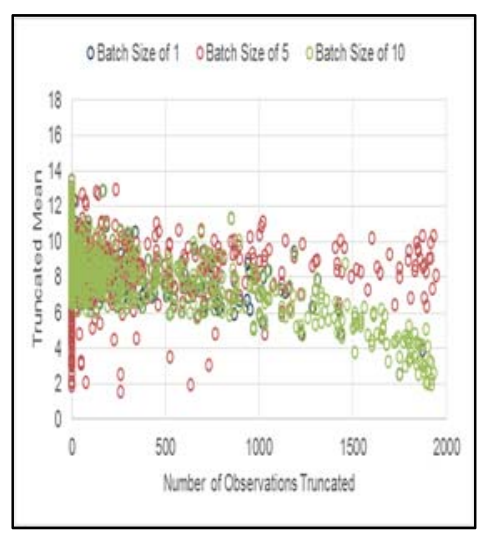

$n=2000$
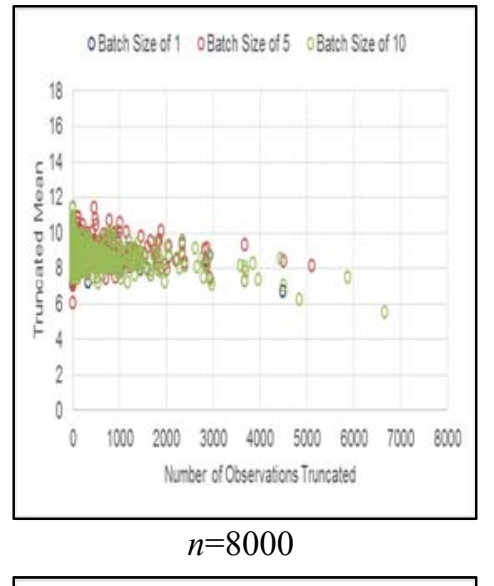

OBatch Sze of 1 OBatch Sze of 5 OBach Sze of 10

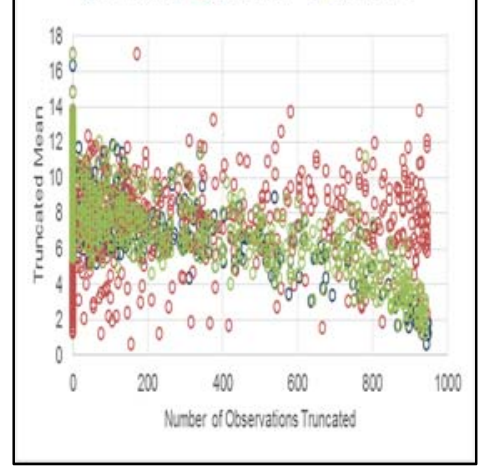

$n=1000$ $n=8000$

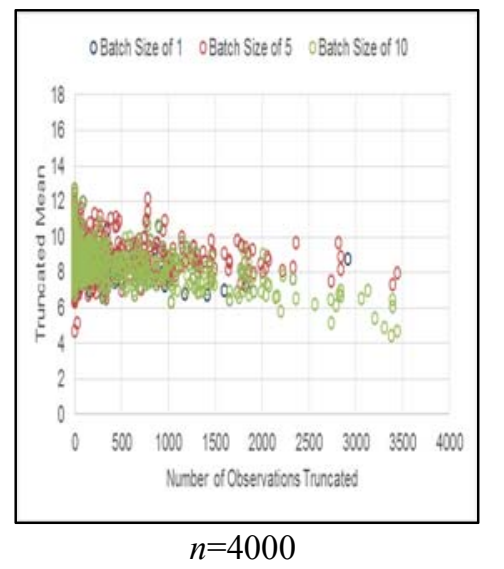

Figure 7.38 Scatterplots of the truncated mean vs. the number of observations truncated for batch sizes $b=1,5$, and 10 for run length of $n=1000,2000,4000,8000$, and 16,000 Model 2 with traffic intensity of $\mathbf{0 . 6 0}$ 
Table 7.11 Correlation between the truncated mean and the number of observations truncated for batch sizes $b=1,5$, and 10 for run length of $n=1000,2000,4000,8000$, and 16,000 for Model 2 with traffic intensity of 0.60

\begin{tabular}{|l|l|l|l|}
\hline & $\begin{array}{l}\text { Trunc } \\
\text { Batch } \\
=1\end{array}$ & $\begin{array}{l}\text { Trunc } \\
\text { Batch } \\
=5\end{array}$ & $\begin{array}{l}\text { Trunc } \\
\text { Batch } \\
=10\end{array}$ \\
\hline $\begin{array}{l}\text { Mean } \\
\text { Batch } \\
=1\end{array}$ & -0.1597 & -0.1056 & -0.0320 \\
\hline $\begin{array}{l}\text { Mean } \\
\text { Batch } \\
=5\end{array}$ & -0.1599 & -0.2199 & -0.1122 \\
\hline $\begin{array}{l}\text { Mean } \\
\text { Batch } \\
=10\end{array}$ & -0.1553 & -0.2128 & -0.2509 \\
\hline
\end{tabular}

\begin{tabular}{|l|l|l|l|}
\hline & $\begin{array}{l}\text { Trunc } \\
\text { Batch } \\
=1\end{array}$ & $\begin{array}{l}\text { Trunc } \\
\text { Batch } \\
=5\end{array}$ & $\begin{array}{l}\text { Trunc } \\
\text { Batch } \\
=10\end{array}$ \\
\hline $\begin{array}{l}\text { Mean } \\
\text { Batch } \\
=1\end{array}$ & -0.2120 & -0.1465 & -0.0421 \\
\hline $\begin{array}{l}\text { Mean } \\
\text { Batch } \\
=5\end{array}$ & -0.2130 & -0.2720 & -0.1367 \\
\hline $\begin{array}{l}\text { Mean } \\
\text { Batch } \\
=10\end{array}$ & -0.1965 & -0.2565 & -0.3522 \\
\hline
\end{tabular}

\begin{tabular}{|l|l|l|l|}
\hline & $\begin{array}{l}\text { Trunc } \\
\text { Batch } \\
=1\end{array}$ & $\begin{array}{l}\text { Trunc } \\
\text { Batch } \\
=5\end{array}$ & $\begin{array}{l}\text { Trunc } \\
\text { Batch } \\
=10\end{array}$ \\
\hline $\begin{array}{l}\text { Mean } \\
\text { Batch } \\
=1\end{array}$ & -0.2819 & -0.1425 & -0.1271 \\
\hline $\begin{array}{l}\text { Mean } \\
\text { Batch } \\
=5\end{array}$ & -0.2939 & -0.4185 & -0.3418 \\
\hline $\begin{array}{l}\text { Mean } \\
\text { Batch } \\
=10\end{array}$ & -0.2920 & -0.4008 & -0.5182 \\
\hline
\end{tabular}

$$
n=16000
$$

$$
n=8000
$$

$n=4000$

\begin{tabular}{|l|l|l|l|}
\hline & $\begin{array}{l}\text { Trunc } \\
\text { Batch } \\
=1\end{array}$ & $\begin{array}{l}\text { Trunc } \\
\text { Batch } \\
=5\end{array}$ & $\begin{array}{l}\text { Trunc } \\
\text { Batch } \\
=10\end{array}$ \\
\hline $\begin{array}{l}\text { Mean } \\
\text { Batch } \\
=1\end{array}$ & -0.4056 & -0.1680 & -0.0967 \\
\hline $\begin{array}{l}\text { Mean } \\
\text { Batch } \\
=5\end{array}$ & -0.3121 & -0.6541 & -0.4410 \\
\hline $\begin{array}{l}\text { Mean } \\
\text { Batch } \\
=10\end{array}$ & -0.2541 & -0.5291 & -0.7204 \\
\hline
\end{tabular}

$$
n=2000
$$

\begin{tabular}{|l|l|l|l|}
\hline & $\begin{array}{l}\text { Trunc } \\
\text { Batch } \\
=1\end{array}$ & $\begin{array}{l}\text { Trunc } \\
\text { Batch } \\
=5\end{array}$ & $\begin{array}{l}\text { Trunc } \\
\text { Batch } \\
=10\end{array}$ \\
\hline $\begin{array}{l}\text { Mean } \\
\text { Batch } \\
=1\end{array}$ & -0.6309 & -0.3548 & -0.2981 \\
\hline $\begin{array}{l}\text { Mean } \\
\text { Batch } \\
=5\end{array}$ & -0.4903 & -0.7493 & -0.5802 \\
\hline $\begin{array}{l}\text { Mean } \\
\text { Batch } \\
=10\end{array}$ & -0.4631 & -0.6305 & -0.7329 \\
\hline
\end{tabular}

$$
n=1000
$$


-Batch Size of 1 - Batch Size of 5 Batch Size of 10

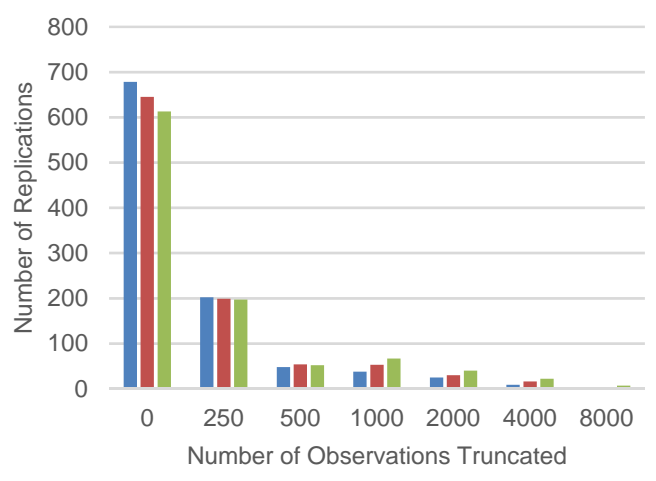

$$
n=16000
$$

- Batch Size of 1 - Batch Size of 5 Batch Size of 10

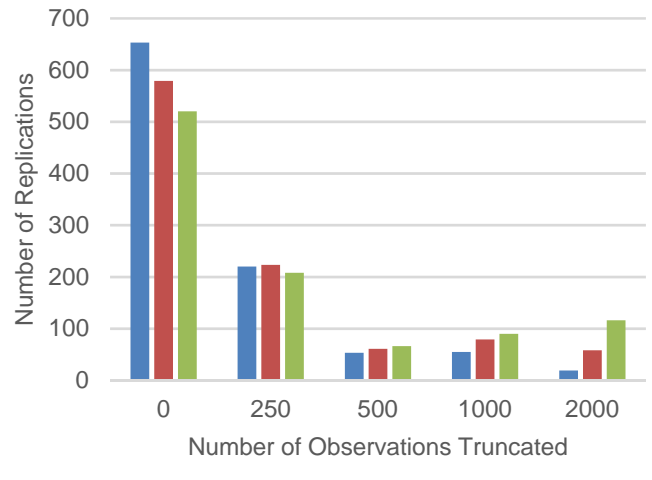

$n=2000$
- Batch Size of 1 - Batch Size of 5 Batch Size of 10

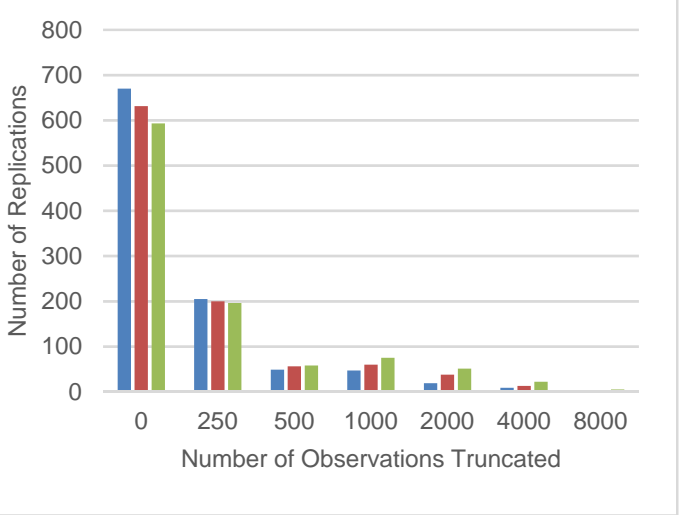

$n=8000$

- Batch Size of 1 Batch Size of 5 Batch Size of 10

700

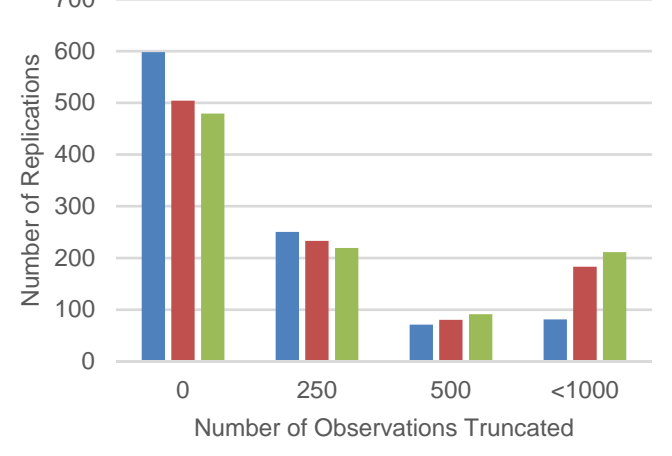

- Batch Size of 1 - Batch Size of 5 - Batch Size of 10

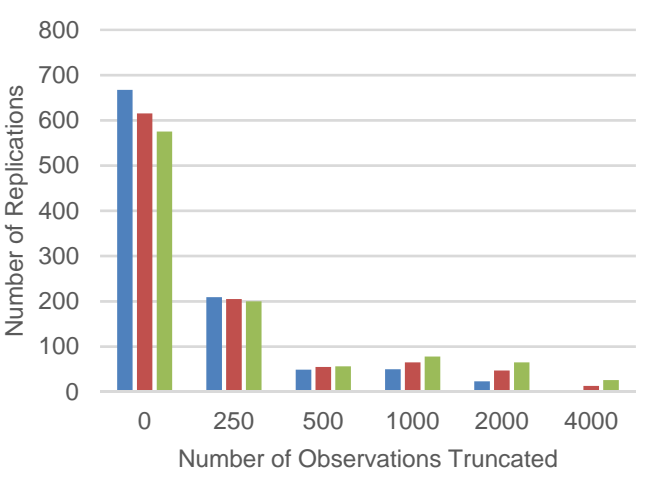

$n=4000$

$$
n=1000
$$$$
n=500
$$

Figure 7.39 Frequency distribution of the number of observations truncated as a function of batch sizes $b=1,5$, and 10 for run length of $n=1000,2000,4000,8000$, and 16,000 for Model 2 with traffic intensity of 0.60 


\subsubsection{Result for Model 2: Simulation run length effect}

With the traffic intensity the same, correlations are plotted against five different run lengths in Figure 7.40 (note that for $\rho=0.9$ the scale for run length differs from the other panels to allow visualization). The grey bars denote approximate inflection points in these graphs. For example, for traffic intensity $\rho=0.9$, the correlations decreases monotonically from $n=8,000$ to $n=64,000$ and then increases monotonically $n=8,000$ to $n=1,000$ for all three batch sizes, with smaller batch sizes generally associated with lesser correlation for all run lengths. As we saw for Model 1, insufficient run lengths appear to be associated with increasing correlations. Because two traffic intensities require shorter run lengths to converge to the steady-state mean, the bars move to the right as traffic intensities decrease.

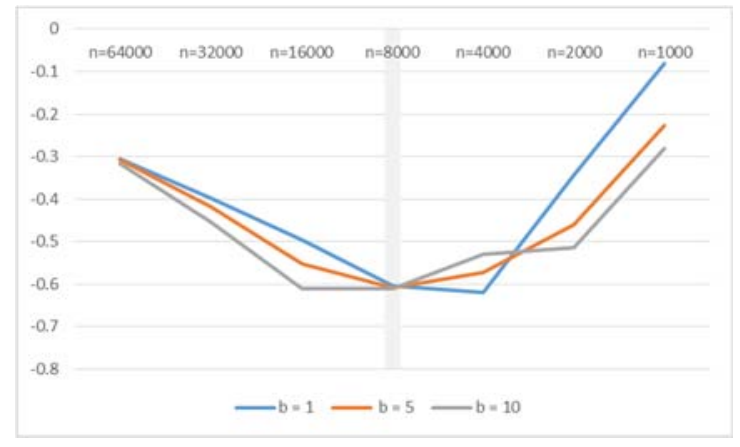

$$
\rho=0.9
$$

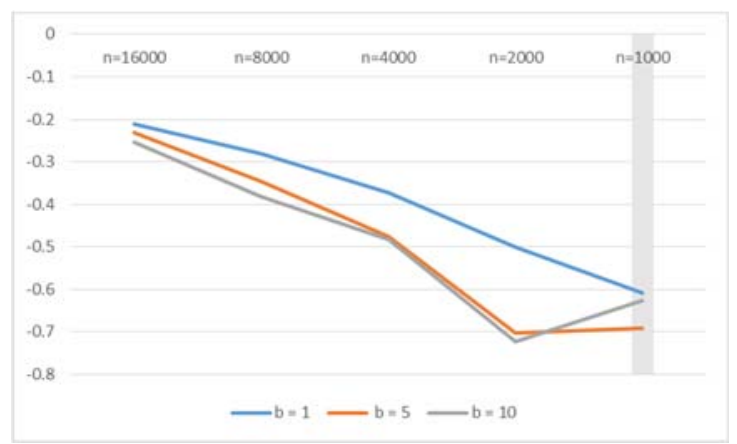

$$
\rho=0.7
$$

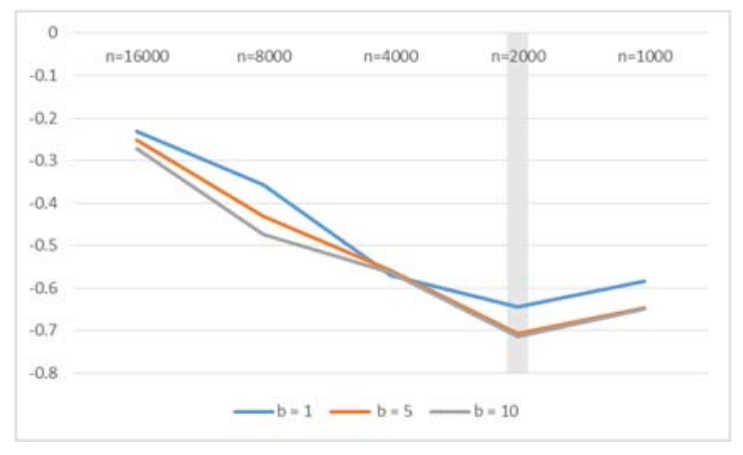

$\rho=0.8$

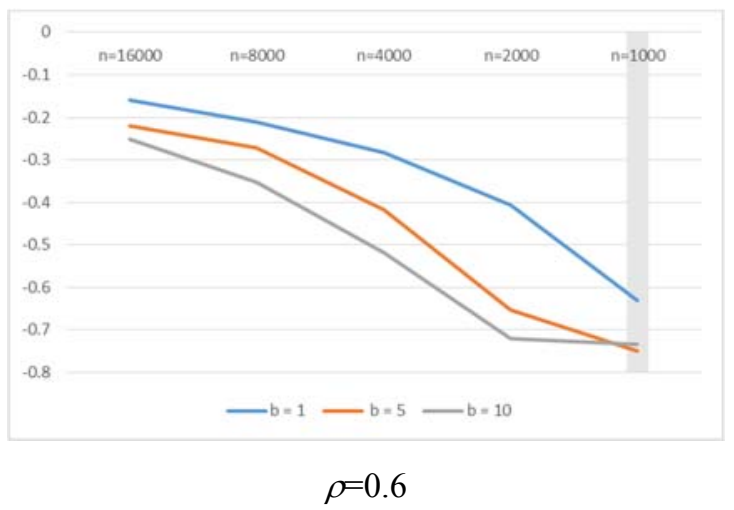

Figure 7.40 Correlation between truncated means and observations truncated as a function of run length and traffic intensity 


\subsubsection{Result for Model 2: Traffic intensity effect}

We also consider how traffic intensities (i.e., 0.6, 0.7, 0.8 and 0.9) affect correlation while the simulation length stays the same.

(1) $n=16000$

Under the same condition of simulation lengths, correlations from the smaller traffic intensities tend to become weaker. That is applicable for all three batch sizes tested.

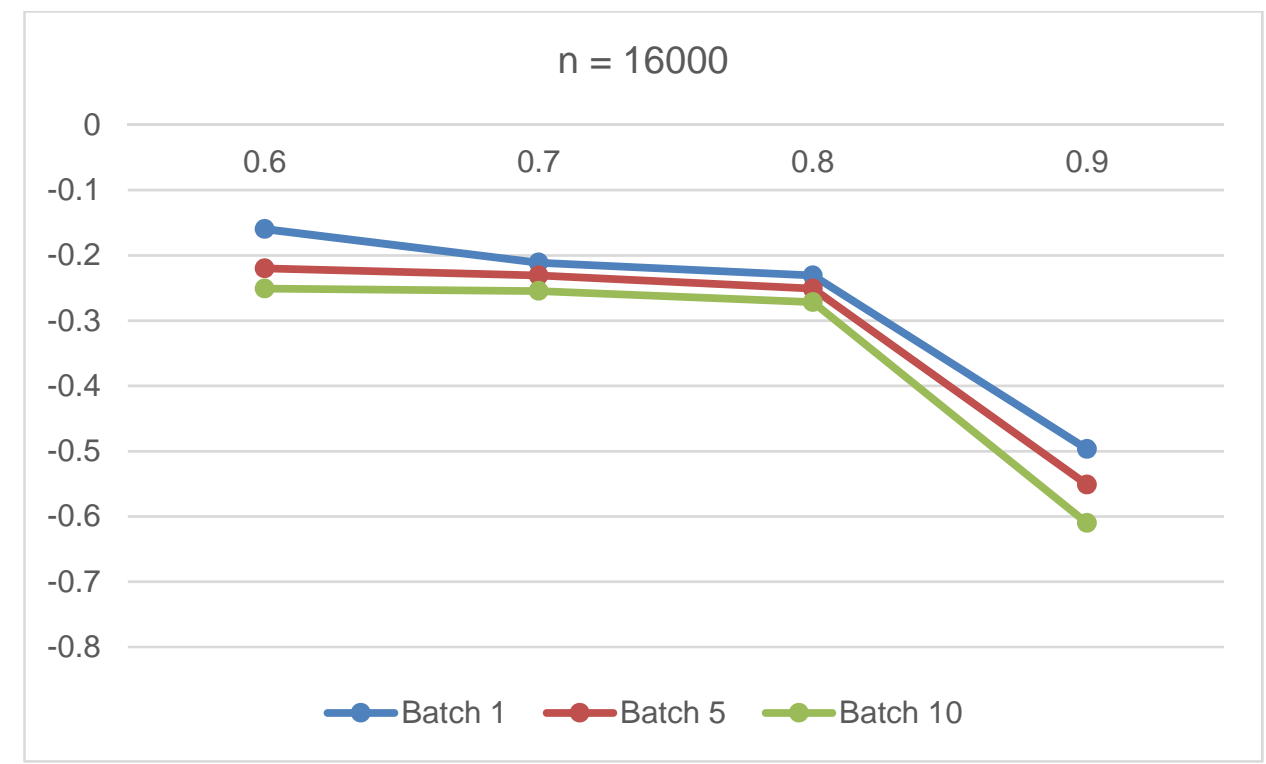

Figure 7.41 Correlation between truncated means and observations truncated by different traffic intensity (Run length of 16000)

(2) $n=8000,4000,2000$, and 1000

The same patterns are shown in the case of $n=8000$ compared to $n=16000$. However, the run lengths of 2000 and 1000 do not conform the same trends as the run lengths of 16000 and 8000 , while $n=4000$ shows mixed trends between the two groups where $n$ is 16000 and 8000 , vs. $n$ is 2000 and 1000 .

We note that the run lengths of 16000 and 8000 ascertain that the longer run length can mitigate influences of traffic intensities except for traffic intensity of 0.9 , regardless of batch sizes. Thus, we can conclude that traffic intensity of 0.9 would benefit from increasing the 
run length. For the run lengths of 2000 and 1000, the correlations between truncated means and observations truncated appear to be counterintuitive as the higher traffic intensity is associated with the lower correlations. However, these trends might be true when long waiting times are infrequently realized during the relatively short simulation runs in the lower traffic intensity such as $0.6,0.7$, and 0.8 . On the other hand, the traffic intensity of 0.9 might build up longer queues even for short runs.
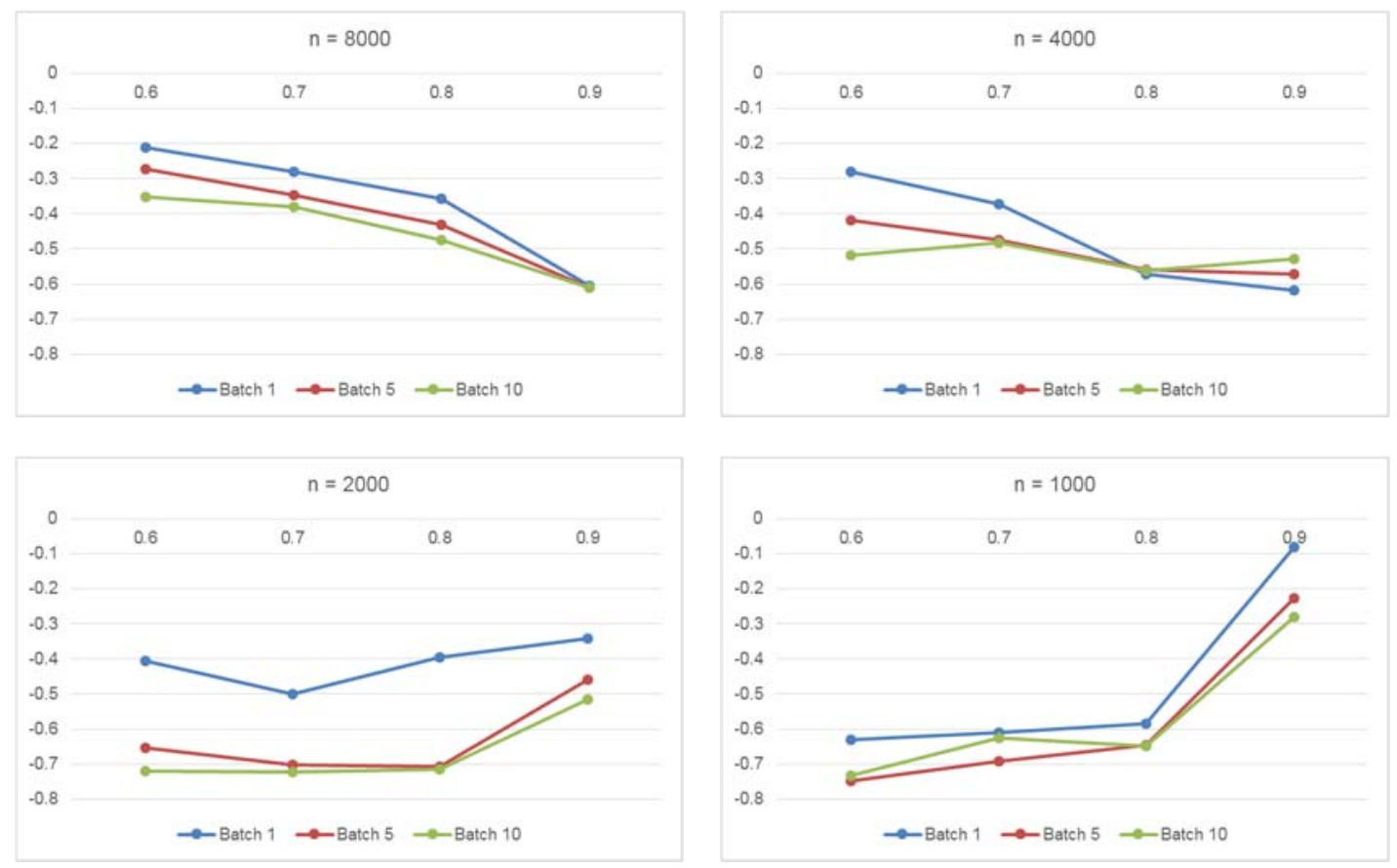

Figure 7.42 Correlation between truncated means and observations truncated by different traffic intensity (Run length of 8000, 4000, 2000, and 100)

\subsubsection{Results: Overlapping Batch Means for M/M/1 with Traffic Intensity of 0.90 .}

We applied OBM to Model 2 with run length $n=64000$ with the highest traffic intensity of 0.9 among $\mathrm{M} / \mathrm{M} / 1$ models for batch sizes $b=10,50,100$, and 200 observation and Figure 7.43 shows four representative cases with original outputs, OBM, and MSER statistic. By increasing batch sizes of OBM, we observed different trends among the four tested cases: 
- Small batch size of 10: The result is almost identical to NOBM because the preprocessed net output series takes closest resemblance for an original output series.

- Batch sizes larger than 10 (i.e., 50, 100, and 200): Based on the results, the difference from a theoretical mean value becomes apparent by increasing batch sizes. This finding can be attributable to (1) regenerative cycles in $\mathrm{M} / \mathrm{M} / 1$ and (2) irregular peaks to influence multiple OBM. Impacts from any outliers in waiting time can remain strong because OBM keeps using these values multiple times compared to NOBM's one usage and go property that any big or small number can influence only one batch mean.

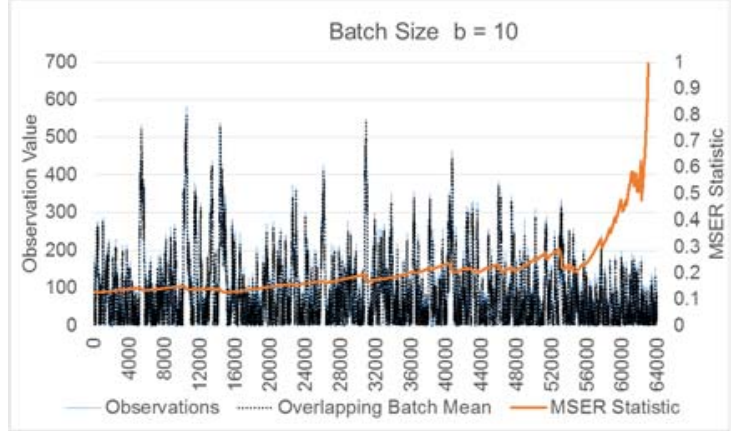

$b=10$

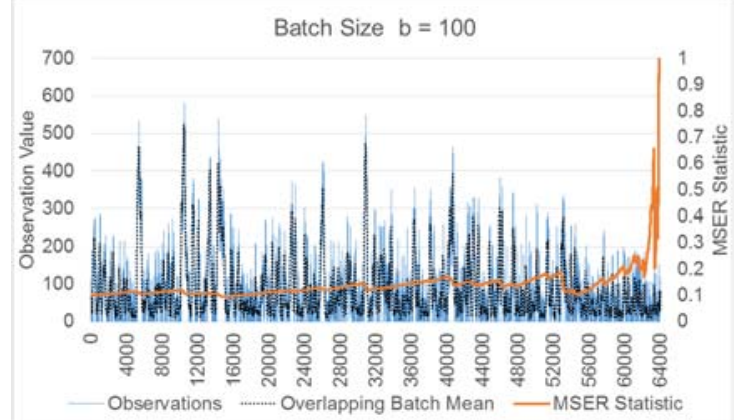

$b=100$
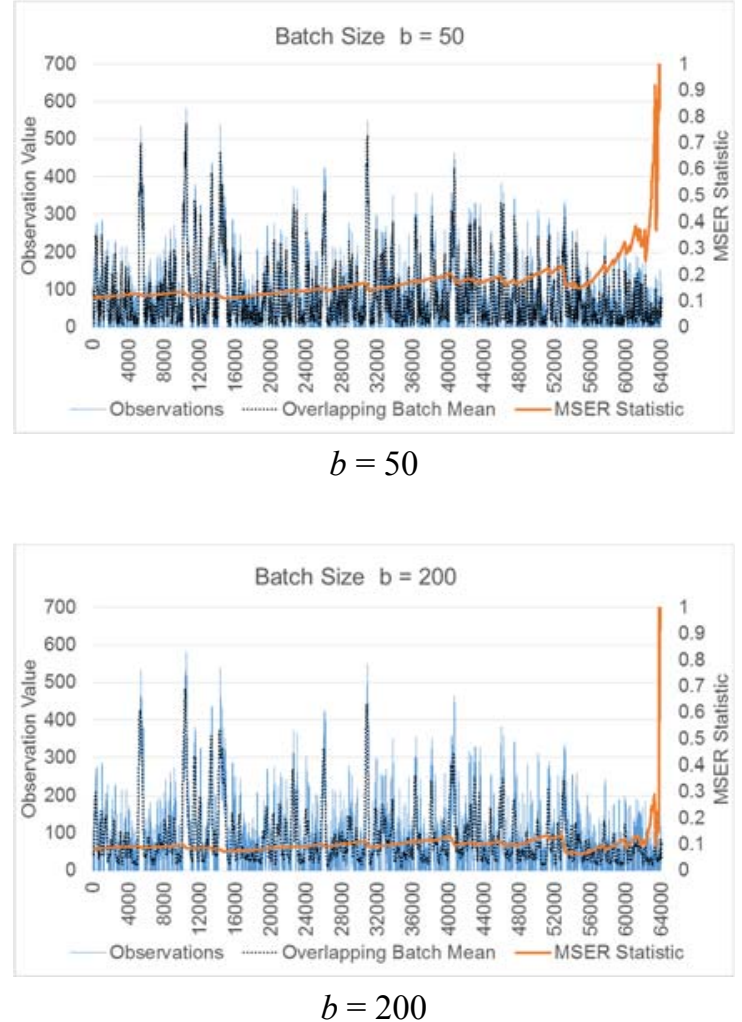

Figure 7.43 Representative Output, Overlapping Batch Mean, and MSER Statistic of Model 2 with traffic intensity of $0.90(b=10,50,100$, and 200) 
We summarize the confidence intervals of the mean estimates and truncation points in Table 7.12 and Figure 7.44 that accounts for our finding. For Model 1 and 3 (following section), OBM tends to outperform NOBM because both cases the output clearly converges to certain mean estimates. However, Models 2 here provides another aspects to be considered. As long as the output takes on a regenerative pattern, OBM might lead to a wrong conclusion about mean estimate for testing models.

Table 7.12 95\% confidence intervals for Model 2 on the truncated mean and the standard deviation for overlapping and non-overlapping batches

\begin{tabular}{|c|c|c|c|c|c|}
\hline & \multicolumn{4}{|c|}{ OBM } & \multirow{2}{*}{$\frac{\mathrm{NOBM}}{\mathrm{NOBM} b=10}$} \\
\hline & OBM $b=10$ & OBM $b=50$ & OBM $b=100$ & $\mathrm{OBM} b=200$ & \\
\hline Sample Mean & 79.162030 & 76.821921 & 74.199907 & 74.199907 & 79.310097 \\
\hline Upper limit & 79.635371 & 77.633984 & 75.211400 & 75.211400 & 79.736781 \\
\hline Lower limit & 78.688690 & 76.009859 & 73.188415 & 73.188415 & 78.883414 \\
\hline Sample Std D & 7.627800 & 13.086239 & 16.300019 & 16.300019 & 6.875926 \\
\hline Upper limit & 7.977651 & 13.686444 & 17.047626 & 17.047626 & 7.191293 \\
\hline Lower limit & 7.307526 & 12.536779 & 15.615620 & 15.615620 & 6.587222 \\
\hline
\end{tabular}

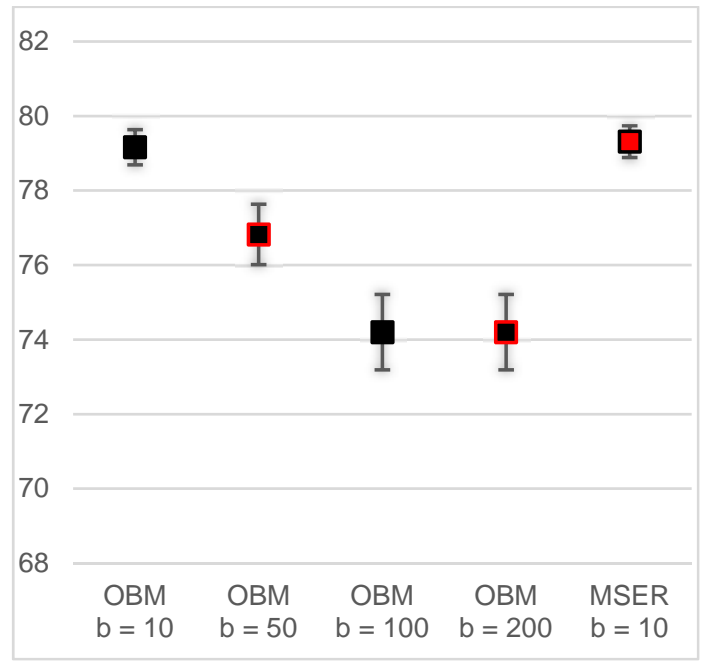

(a)

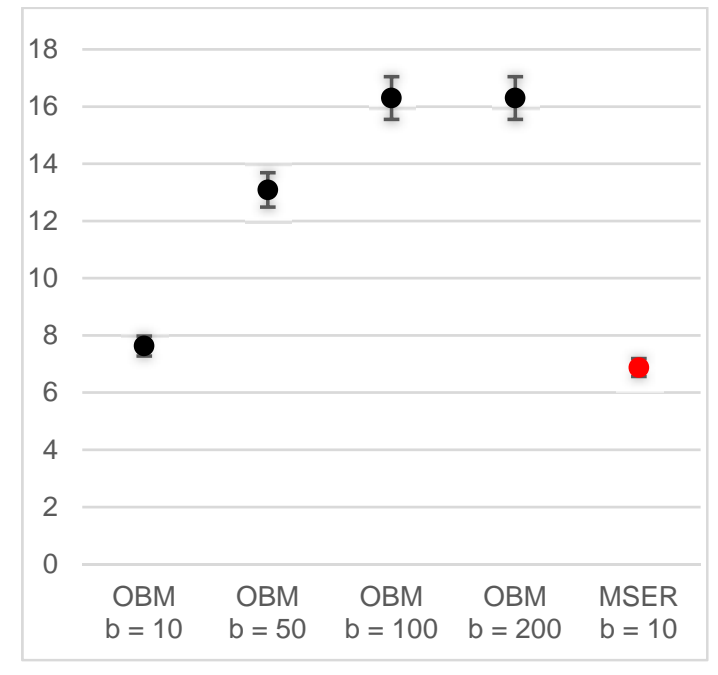

(b)

Figure 7.44 95\% confidence intervals for Model 3 on (a) the truncated mean for overlapping and non-overlapping batches and (b) the standard deviation for overlapping and nonoverlapping batches 
Figure 7.45 and 7.46 and Table 7.13 below reiterate the inefficiency of bigger OBM to find accurate mean estimate and significant loss in output series is observed since truncation points pass the first half even with run length of 64000. That is, "batch size of 200" indeed uses less than $10 \%$ of total output and estimates quite smaller mean estimates.

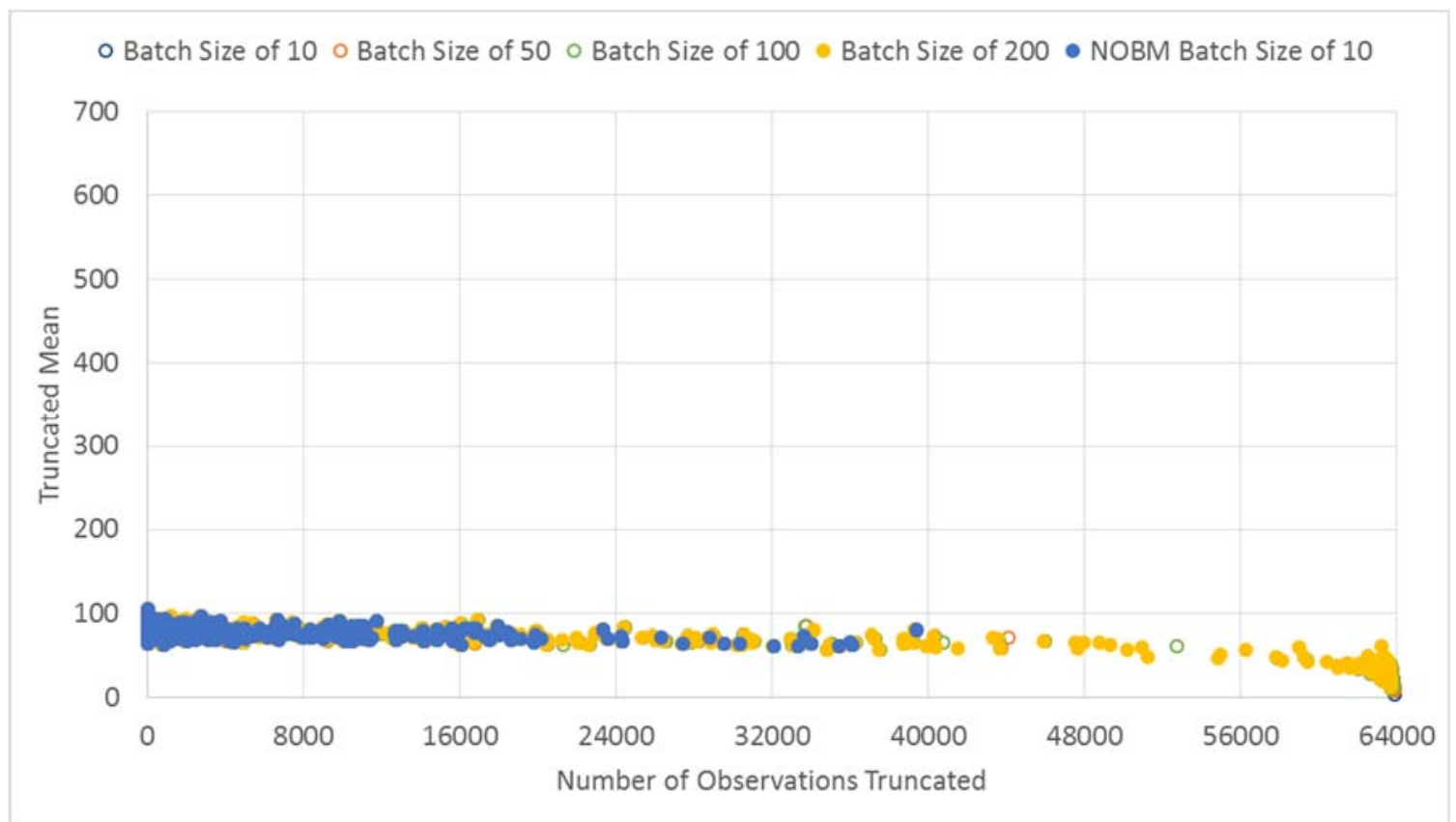

Figure 7.45 Scatterplots of the truncated mean vs. the number of observations truncated for OBM sizes $b=10,50,100$ and 200 for run length of $n=64,000$ with traffic intensity of 0.90 for Model 2 (including NOBM batch size of 10) 


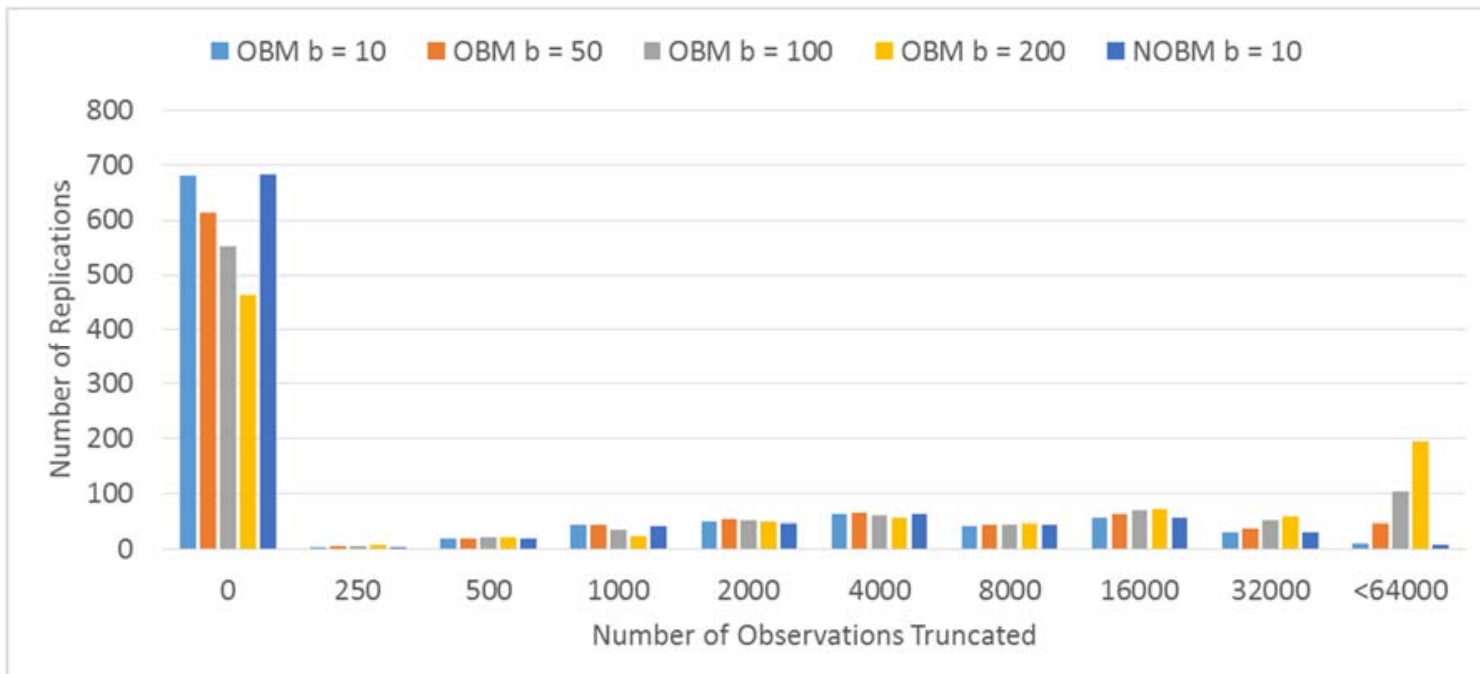

Figure 7.46 Frequency distribution of the number of observations truncated as a function of OBM sizes $b=10,50,100$, and 200 for run length of $n=64,000$ with traffic intensity of 0.90 for Model 2 (including NOBM batch size of 10)

Table 7.13 Correlation between the truncated mean and the number of observations truncated for OBM sizes $b=10,50,100$, and 200 for run length of $n=64,000$ for Model 2

\begin{tabular}{|l|c|c|c|c|}
\hline & Trunc Batch =10 & Trunc Batch =50 & Trunc Batch =100 & Trun Batch = 200 \\
\hline Mean Batch $=10$ & 0.043 & -0.053 & -0.890 & 0.082 \\
\hline Mean Batch $=50$ & -0.198 & -0.829 & -0.299 & -0.114 \\
\hline Mean Batch $=100$ & -0.120 & -0.333 & -0.890 & 0.017 \\
\hline Mean Batch $=200$ & -0.113 & -0.210 & -0.422 & -0.914 \\
\hline
\end{tabular}

As we observe, without applying $d^{*}<n / 2$ rule, the results for OBM have been deteriorated compared to NOBM as well as raw output series without any truncation. Thus, we try to capture the characteristics of truncations satisfying $d^{*}<n / 2$ rule for OBM and the results are summarized in Table 7.14 and Figure 7.45. The disadvantage following this rule is that some of replications need to be omitted to obtain mean estimates. First, the set of mean estimates are tabulated according to different run lengths from $n=64000$ to 1000 . Short lengths of $n$ such as 1000,2000 , and 4000 do not show accuracy of means compared to NOBM estimates but longer run lengths apparently show improved convergence to a theoretical estimate. 
Table7.14 Confidence Intervals for $M / M / 1$ with traffic intensity of 0.90 on the truncated mean and the standard deviation for overlapping and non-overlapping batches with traffic intensity of $0.90(n=1000,2000,4000,8000,16000,32000$, and 64000)

\begin{tabular}{|c|c|c|c|c|c|}
\hline$n=64000$ & \multicolumn{4}{|c|}{ OBM } & NOBM \\
\hline Mean with truncation & Batch Size of 10 & Batch Size of 50 & Batch Size of 100 & Batch Size of 200 & Batch Size of 10 \\
\hline Sample Mean & 79.42 & 79.09 & 78.85 & 78.42 & 79.42 \\
\hline Upper limit & 79.84 & 79.51 & 79.29 & 78.87 & 79.84 \\
\hline Lower limit & 79.00 & 78.66 & 78.41 & 77.97 & 79.00 \\
\hline Sample Std Dev & 6.77 & 6.68 & 6.67 & 6.53 & 6.77 \\
\hline Upper limit & 7.09 & 6.98 & 6.98 & 6.83 & 7.08 \\
\hline Lower limit & 6.49 & 6.40 & 6.39 & 6.25 & 6.49 \\
\hline
\end{tabular}

\begin{tabular}{|c|c|c|c|c|c|}
\hline$n=32000$ & \multicolumn{4}{|c|}{ OBM } & NOBM \\
\hline Mean with truncation & Batch Size of 10 & Batch Size of 50 & Batch Size of 100 & Batch Size of 200 & Batch Size of 10 \\
\hline Sample Mean & 78.28 & 77.73 & 77.22 & 76.55 & 78.37 \\
\hline Upper limit & 78.85 & 78.32 & 77.84 & 77.22 & 78.93 \\
\hline Lower limit & 77.71 & 77.13 & 76.59 & 75.89 & 77.80 \\
\hline Sample Std Dev & 8.95 & 8.89 & 8.91 & 8.69 & 8.99 \\
\hline Upper limit & 9.36 & 9.30 & 9.31 & 9.08 & 9.41 \\
\hline Lower limit & 8.57 & 8.51 & 8.53 & 8.32 & 8.62 \\
\hline
\end{tabular}

\begin{tabular}{|c|c|c|c|c|c|}
\hline$n=16000$ & \multicolumn{4}{|c|}{ OBM } & NOBM \\
\hline Mean with truncation & Batch Size of 10 & Batch Size of 50 & Batch Size of 100 & Batch Size of 200 & Batch Size of 10 \\
\hline Sample Mean & 76.64 & 76.16 & 75.82 & 75.98 & 76.72 \\
\hline Upper limit & 77.47 & 77.07 & 76.80 & 77.07 & 77.53 \\
\hline Lower limit & 75.82 & 75.25 & 74.84 & 74.89 & 75.91 \\
\hline Sample Std Dev & 12.63 & 12.71 & 12.68 & 12.79 & 12.60 \\
\hline Upper limit & 13.21 & 13.29 & 13.26 & 13.38 & 13.18 \\
\hline Lower limit & 12.10 & 12.17 & 12.15 & 12.25 & 12.07 \\
\hline
\end{tabular}

\begin{tabular}{|c|c|c|c|c|c|}
\hline$n=8000$ & \multicolumn{4}{|c|}{ OBM } & NOBM \\
\hline Mean with truncation & Batch Size of 10 & Batch Size of 50 & Batch Size of 100 & Batch Size of 200 & Batch Size of 10 \\
\hline Sample Mean & 72.13 & 71.93 & 72.13 & 71.85 & 72.78 \\
\hline Upper limit & 73.20 & 73.15 & 73.52 & 73.41 & 73.83 \\
\hline Lower limit & 71.06 & 70.71 & 70.74 & 70.28 & 71.72 \\
\hline Sample Std Dev & 15.48 & 15.30 & 15.63 & 15.90 & 15.84 \\
\hline Upper limit & 16.19 & 16.00 & 16.34 & 16.63 & 16.56 \\
\hline Lower limit & 14.83 & 14.65 & 14.97 & 15.23 & 15.17 \\
\hline
\end{tabular}




\begin{tabular}{|c|c|c|c|c|c|}
\hline$n=4000$ & \multicolumn{4}{|c|}{ OBM } & NOBM \\
\hline Mean with truncation & Batch Size of 10 & Batch Size of 50 & Batch Size of 100 & Batch Size of 200 & Batch Size of 10 \\
\hline Sample Mean & 69.21 & 69.47 & 68.50 & 68.91 & 70.50 \\
\hline Upper limit & 70.70 & 71.21 & 70.34 & 70.85 & 71.97 \\
\hline Lower limit & 67.71 & 67.72 & 66.67 & 66.97 & 69.03 \\
\hline Sample Std Dev & 20.39 & 20.87 & 20.32 & 20.15 & 21.50 \\
\hline Upper limit & 21.33 & 21.83 & 21.25 & 21.08 & 22.48 \\
\hline Lower limit & 19.53 & 20.00 & 19.47 & 19.31 & 20.59 \\
\hline
\end{tabular}

\begin{tabular}{|c|c|c|c|c|c|}
\hline$n=2000$ & \multicolumn{4}{|c|}{ OBM } & NOBM \\
\hline Mean with truncation & Batch Size of 10 & Batch Size of 50 & Batch Size of 100 & Batch Size of 200 & Batch Size of 10 \\
\hline Sample Mean & 64.64 & 65.23 & 65.97 & 67.59 & 66.16 \\
\hline Upper limit & 66.55 & 67.75 & 68.74 & 70.33 & 68.15 \\
\hline Lower limit & 62.73 & 62.71 & 63.21 & 64.86 & 64.16 \\
\hline Sample Std Dev & 24.71 & 27.95 & 28.78 & 28.11 & 27.06 \\
\hline Upper limit & 25.84 & 29.24 & 30.10 & 29.39 & 28.30 \\
\hline Lower limit & 23.67 & 26.78 & 27.57 & 26.93 & 25.93 \\
\hline
\end{tabular}

\begin{tabular}{|c|c|c|c|c|c|}
\hline$n=1000$ & \multicolumn{4}{|c|}{ OBM } & NOBM \\
\hline Mean with truncation & Batch Size of 10 & Batch Size of 50 & Batch Size of 100 & Batch Size of 200 & Batch Size of 10 \\
\hline Sample Mean & 63.58 & 66.00 & 66.41 & 69.56 & 68.07 \\
\hline Upper limit & 66.69 & 69.76 & 70.34 & 73.68 & 71.58 \\
\hline Lower limit & 60.46 & 62.24 & 62.48 & 65.44 & 64.55 \\
\hline Sample Std Dev & 39.52 & 42.39 & 43.35 & 47.31 & 44.58 \\
\hline Upper limit & 41.33 & 44.33 & 45.34 & 49.47 & 46.63 \\
\hline Lower limit & 37.86 & 40.61 & 41.53 & 45.32 & 42.71 \\
\hline
\end{tabular}




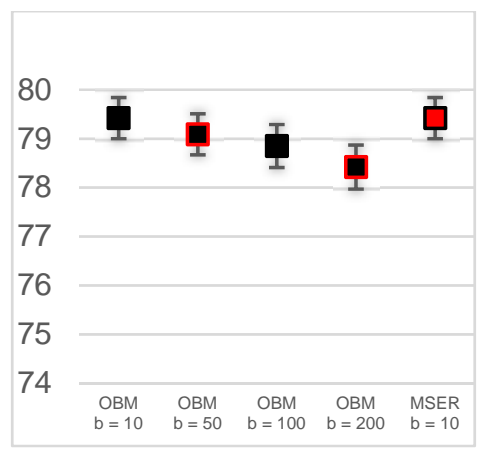

$n=64000$

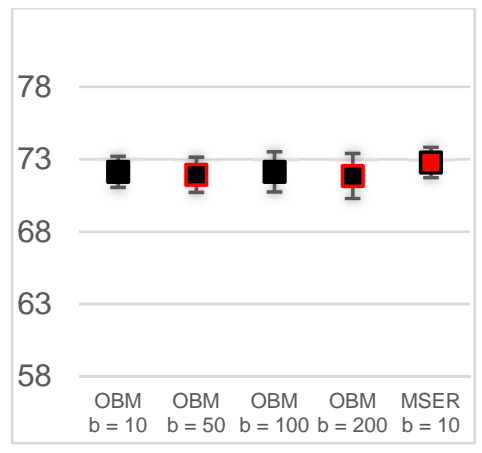

$n=8000$

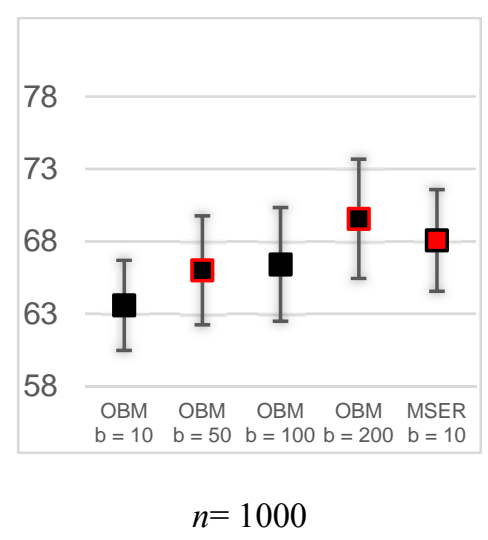

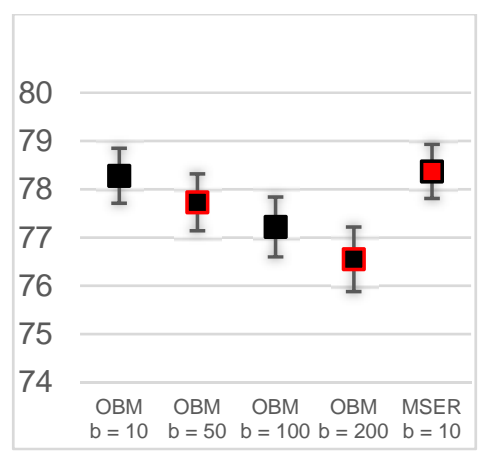

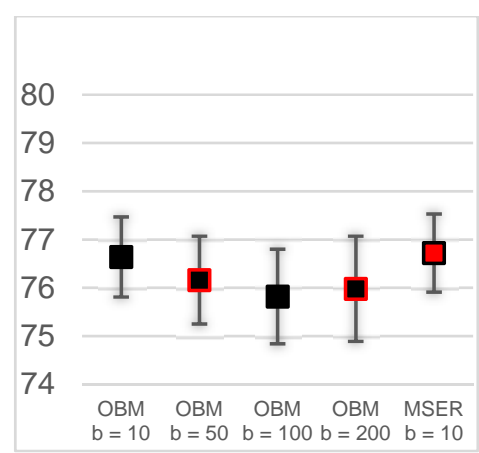

$n=32000$

$$
n=16000
$$
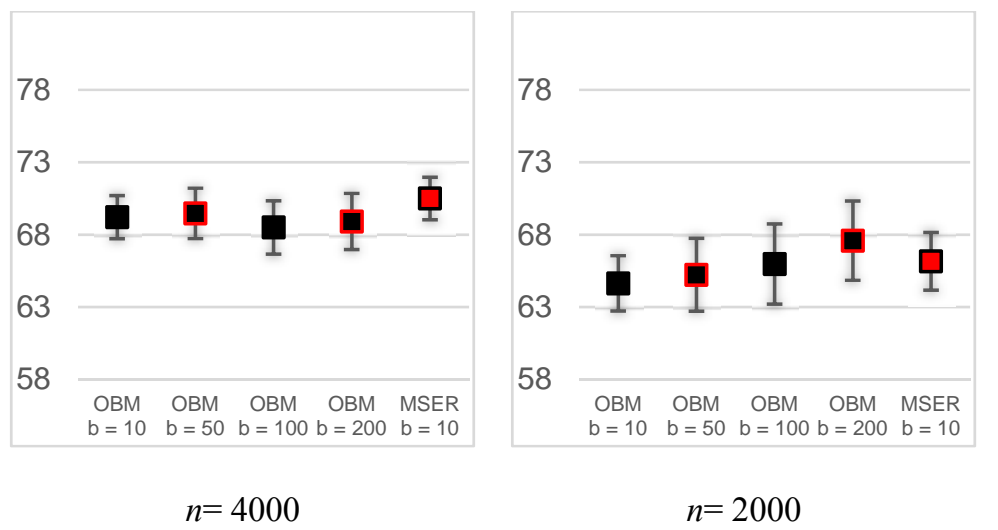

Figure $7.4795 \%$ confidence intervals for $\mathrm{M} / \mathrm{M} / 1$ with traffic intensity of 0.90 about the truncated mean for overlapping and non-overlapping batches $(n=1000,2000,4000,8000$, 16000, 32000, and 64000.) 


\subsubsection{Results: Initialization Bias}

To test the assertion that the errors in the output are the result of inadequate run lengths and unrepresentative initial conditions (note that $x_{0}=0$ is the mode of the steady-state distribution and hardly representative!), we ran two more experiments. Each comprised 100 replications of Model 2 with traffic intensity of 0.9 and initial condition $x_{0}=100$. We used and compared three different batch sizes, $b=1,5$, and 10, and two different run lengths, $n=64,000$ and 32,000 .

Figure 7.48 shows the output time series for one representative replication with $b=1$ and $n=32,000$. Clearly, the initial condition results in a large initial spike in waiting times. Just as clearly, MSER removes much or this entire initial spike.

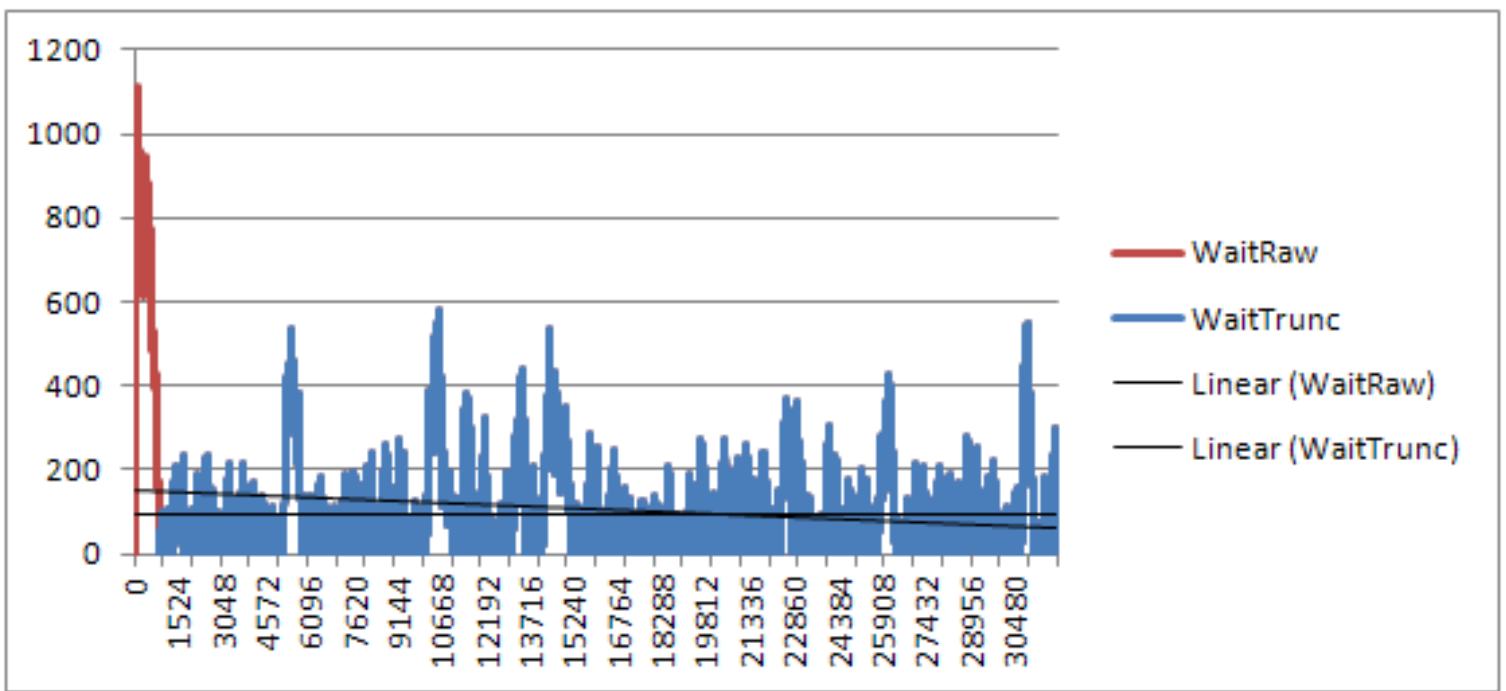

Figure 7.48 Output time series with traffic intensity of 0.90 with $b=1$ and $n=32,000$

In contrast to the prior experiments without initialization bias, MSER truncation provides significantly improved estimates over those obtained without truncation in Figure 7.49. These results appear to be relatively insensitive to batch size (although smaller batches appear to perform modestly better) and, again, the error in the estimates is relatively uncorrelated to the MSER truncation points. The effect of run length is apparent and for 
the longer runs coverage is achieved for every batch size. We speculate that this may be attributable to the significant reduction in sample variance for longer runs.

$95 \%$

confidence intervals on the mean

$95 \%$ confidence intervals on Sample Std Dev.

Scatterplots indicting the correlation between truncated mean and truncation point

Frequency distribution of the number of observations truncated
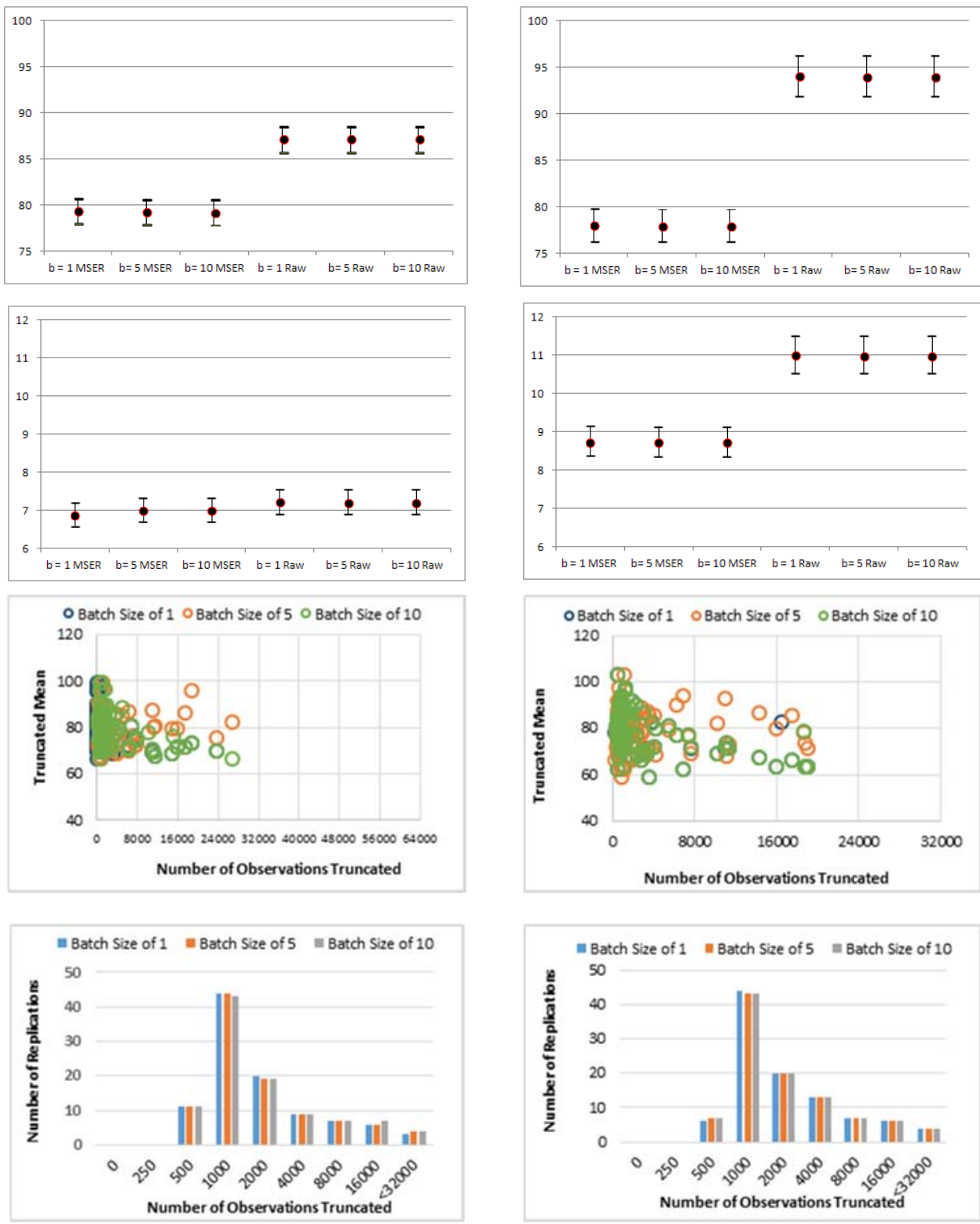

$n=64,000$

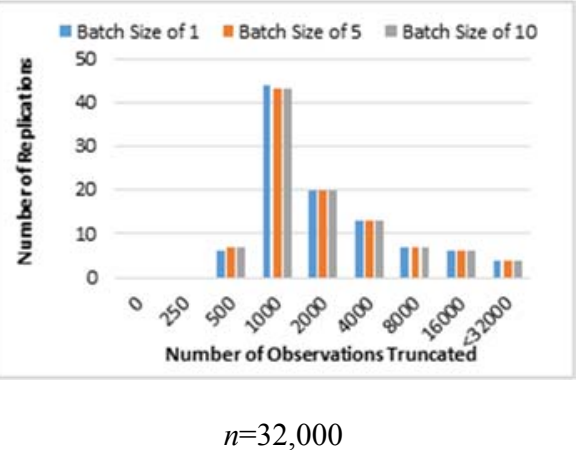

Figure 7.49 Confidence interval for Mean and Standard Deviation, Scatter Plot for Truncation points and Truncated Mean, Frequency Distribution of the Number of Observations Truncated with Traffic Intensity of 0.90 with $b=1,5$, and 10 and $n=64,000$ and 32,000 
Note that this example appears to suggest that "smart initialization" may not be the solution to the warm-up problem that has been widely recommendation. The initial condition of $x_{0}=100$ certainly is much closer to the theoretical steady-state mean of 81 than $x_{0}=0$ in the prior examples. However, truncation is still required to eliminate the initial spike in waiting times. The same would be true if we had made the very lucky guess of $x_{0}=81 !$ Warm-up appears to be required. In this instance smart initialization appears to suggest foreknowledge of the entire steady-state distribution, in particular the mode, rather than the steady state mean.

Table 7.15 Correlation between the truncated mean and the number of observations truncated for $b=1,5$, and 10 for run length of $n=32,000$ with initial bias of 100

\begin{tabular}{|l|c|c|c|}
\hline & Trunc Batch $=1$ & Trunc Batch =5 & Trunc Batch =10 \\
\hline Mean Batch $=1$ & -0.4147 & -0.4050 & -0.4050 \\
\hline Mean Batch $=5$ & -0.4308 & -0.4232 & -0.4233 \\
\hline Mean Batch $=10$ & -0.4308 & -0.4232 & -0.4233 \\
\hline
\end{tabular}

Table 7.16 95\% confidence intervals for Model 2 on the observation truncated and the standard deviation for run length of $n=32,000$ with initial bias of 100

\begin{tabular}{|l|r|r|r|}
\hline Truncation Point & batch $=1$ & batch $=5$ & batch $=10$ \\
\hline Sample Mean & 2878.4400 & 2900.7000 & 2901.3000 \\
\hline Upper limit & 3735.0552 & 3772.1267 & 3772.7876 \\
\hline Lower limit & 2021.8248 & 2029.2733 & 2029.8124 \\
\hline Sample Std Dev & 4317.1446 & 4391.7915 & 4392.0984 \\
\hline Upper limit & 4515.1520 & 4593.2226 & 4593.5436 \\
\hline Lower limit & 4135.8779 & 4207.3905 & 4207.6845 \\
\hline
\end{tabular}

Table 7.17 95\% confidence intervals for Model 2 on the truncated mean and the standard deviation for run length of $n=32,000$ with initial bias of 100

\begin{tabular}{|l|r|r|r|}
\hline \multicolumn{1}{|c|}{ Mean with truncation } & batch $=1$ & batch $=5$ & batch $=10$ \\
\hline Sample Mean & 78.036849 & 77.993410 & 77.992041 \\
\hline Upper limit & 79.770945 & 79.725121 & 79.723891 \\
\hline Lower limit & 76.302752 & 76.261699 & 76.260191 \\
\hline Sample Std Dev & 8.739450 & 8.727426 & 8.728127 \\
\hline Upper limit & 9.140288 & 9.127712 & 9.128446 \\
\hline Lower limit & 8.372501 & 8.360982 & 8.361653 \\
\hline
\end{tabular}


Table7.18 95\% confidence intervals for Model 2 on the mean without truncation and the standard deviation for run length of $n=32,000$ with initial bias of 100

\begin{tabular}{|l|r|r|r|}
\hline Mean w/o truncation & batch $=1$ & batch $=5$ & batch $=10$ \\
\hline Sample Mean & 94.087628 & 94.0876 & 94.0876 \\
\hline Upper limit & 96.269303 & 96.2693 & 96.2693 \\
\hline Lower limit & 91.905953 & 91.9060 & 91.9060 \\
\hline Sample Std Dev & 10.995146 & 10.9951 & 10.9951 \\
\hline Upper limit & 11.499442 & 11.4994 & 11.4994 \\
\hline Lower limit & 10.533485 & 10.5335 & 10.5335 \\
\hline
\end{tabular}

Table 7.19 Correlation between the truncated mean and the number of observations truncated for $b=1,5$, and 10 for run length of $n=64,000$ with initial bias of 100

\begin{tabular}{|l|l|l|l|}
\hline & Trunc Batch =1 & Trunc Batch =5 & Trunc Batch =10 \\
\hline Mean Batch $=1$ & -0.3940 & -0.3588 & -0.3491 \\
\hline Mean Batch $=5$ & -0.3811 & -0.4295 & -0.4191 \\
\hline Mean Batch $=10$ & -0.3782 & -0.4269 & -0.4268 \\
\hline
\end{tabular}

Table 7.20 95\% confidence intervals for Model 2 on the observation truncated and the standard deviation for run length of $n=64,000$ with initial bias of 100

\begin{tabular}{|l|r|r|r|}
\hline Truncation Point & batch $=1$ & \multicolumn{1}{|c|}{ batch $=5$} & batch $=10$ \\
\hline Sample Mean & 2738.4600 & 2995.2500 & 3092.4000 \\
\hline Upper limit & 3624.6425 & 3999.8582 & 4105.6587 \\
\hline Lower limit & 1852.2775 & 1990.6418 & 2079.1413 \\
\hline Sample Std Dev & 4466.1572 & 5062.9956 & 5106.5923 \\
\hline Upper limit & 4670.9991 & 5295.2117 & 5340.8080 \\
\hline Lower limit & 4278.6337 & 4850.4123 & 4892.1785 \\
\hline
\end{tabular}

Table 7.21 95\% confidence intervals for Model 2 on the truncated mean and the standard deviation for run length of $n=64,000$ with initial bias of 100

\begin{tabular}{|l|r|r|r|}
\hline \multicolumn{1}{|c|}{ Mean with truncation } & batch $=1$ & batch $=5$ & batch $=10$ \\
\hline Sample Mean & 79.395610 & 79.281469 & 79.243732 \\
\hline Upper limit & 80.761263 & 80.670545 & 80.632182 \\
\hline Lower limit & 78.029956 & 77.892394 & 77.855283 \\
\hline Sample Std Dev & 6.882581 & 7.000622 & 6.997469 \\
\hline Upper limit & 7.198253 & 7.321708 & 7.318410 \\
\hline Lower limit & 6.593598 & 6.706682 & 6.703661 \\
\hline
\end{tabular}


Table 7.22 95\% confidence intervals for Model 2 on the mean without truncation and the standard deviation for run length of $n=64,000$ with initial bias of 100

\begin{tabular}{|l|r|r|r|}
\hline \multicolumn{1}{|c|}{ Mean w/o truncation } & batch $=1$ & batch $=5$ & batch $=10$ \\
\hline Sample Mean & 87.175809 & 87.1758 & 87.1758 \\
\hline Upper limit & 88.607643 & 88.6076 & 88.6076 \\
\hline Lower limit & 85.743975 & 85.7440 & 85.7440 \\
\hline Sample Std Dev & 7.216117 & 7.2161 & 7.2161 \\
\hline Upper limit & 7.547086 & 7.5471 & 7.5471 \\
\hline Lower limit & 6.913129 & 6.9131 & 6.9131 \\
\hline
\end{tabular}

\subsubsection{Summary Results for Model 2}

We chose this model initially because it allows comparison with the results obtained by Law (2015) and by White and Hwang (2015) for long runs. This prior research addressed the performance of MSER for a single batch size $(b=5)$, single run length $(n=65,000)$, and single traffic intensity $(\rho=0.9)$ with respect to a range of different initial conditions $\left\{x_{0}=0\right.$, $5,10,12,15,18,20\}$. The results obtained by White and Hwang (2015) demonstrated that truncation points and the error in the truncated mean estimates are essentially independent. Further, while the mean estimates for this problem are quite good without truncation, applying MSER-5 truncation modestly improves the accuracy of these estimates.

(1) The results obtained in the present research for $n=64,000$ and $\rho=0.9$ are entirely consistent with those reported earlier.

Our objective here was to explore the performance of MSER for a single initial condition, empty and idle $\left\{x_{0}=0\right\}$, with respect to alternative run lengths, batch sizes, and model traffic 
intensities. We speculated the effects of decreasing $\rho$ to be similar to those encountered by increasing the run length $n$.

(2) The results of our experiments suggest that this speculation is largely untrue and decreasing traffic intensity cannot altogether compensate for short run lengths.

(3) The $d^{*} \leq d_{\max }=n / 2$ truncation rule likewise cannot altogether compensate for short run lengths

For smaller run lengths and traffic intensities, MSER appears to overestimate the amount truncation warranted, reducing the sample variance by truncating early regenerative cycles with large peak waiting times while the MSER statistic is relatively less sensitive to the accompanying reduction in sample size. The result is underestimation of the steady-state mean.

In the final analysis, our analysis demonstrates that

(4) The difficulty in estimating the steady-state mean has little or nothing to do with bias resulting from a poor choice of initial conditions for Model 2. The empty-and-idle condition regenerates frequently, more frequently for lower traffic intensities and smaller batches.

(5) The fundamental issue is determining an initial run length that is sufficiently long to capture observations that, taken together, are representative of the steady-state distribution.

(6) The new initial bias of $x_{0}=100$ apparently vindicates the efficacy of MSER to detect steady state mean(s) and truncation point(s) compared to smart initialization approach. 


\subsubsection{Model 3: EAR(1)}

The third test model is $\operatorname{AR}(1)$

$$
X_{t}=\phi X_{t-1}+\varepsilon_{t}
$$

for $t=0,1, \ldots, n$, with exponentially decaying error

$$
\varepsilon_{t} \sim \lambda e^{-\lambda t}
$$

where $\lambda=1$. This model differs from a typical AR(1) with normally distributed white noise because the error term is exponentially distributed. It is stable if and only if

$$
0 \leq \phi<1
$$

For stable processes, the expected steady-mean is

$$
E[\bar{X}]=\frac{1}{1-\phi}
$$

Table 7.23 provides this expectation for parameter values $\phi=\{0.7,0.8,0.9,0.99\}$. Note that the rate of convergence to steady state also depends $\phi$, with the rate increasing in decreasing $\phi$. In terms of the average warm-up period required, therefore, we would anticipate that the effect of decreasing $\phi$ is similar to that of increasing the run length $n$.

Table 7.23 Expected Value of the Steady-State Mean as a function of $\phi$

\begin{tabular}{cccccc}
\hline \multicolumn{5}{c}{} & \multicolumn{5}{c}{$\phi$} \\
\cline { 2 - 5 } & $E[\bar{X}]$ & 0.7 & 0.8 & 0.9 & 0.99 \\
\hline & 3.33 & 5.00 & 10 & 100 \\
\hline
\end{tabular}

Both of these dependencies are illustrated in Figure 7.50 for a run length of $n=500$. (Note the scale for the ordinate in the panel for $\phi=0.99$ differs from the other three panels, in order to allow visualization.) In each panel, the response $X_{t}$ is plotted in blue, while the expected response 


$$
E\left[X_{t}\right]=\phi E\left[X_{t-1}\right]+1
$$

is plotted in red.

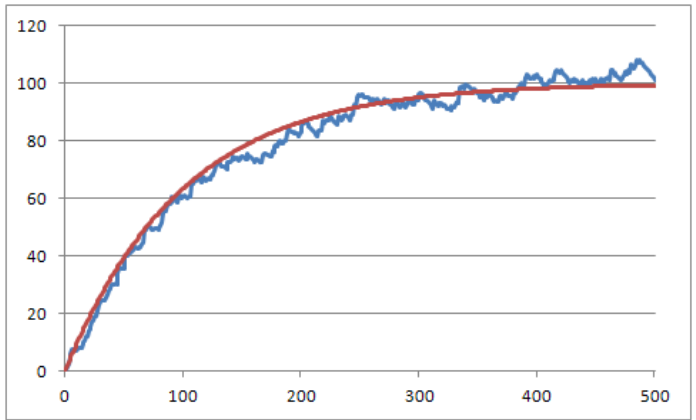

$\phi=0.99$

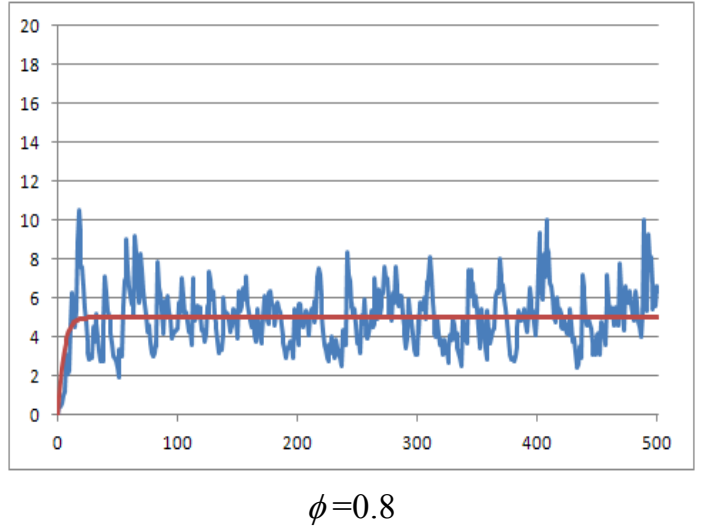

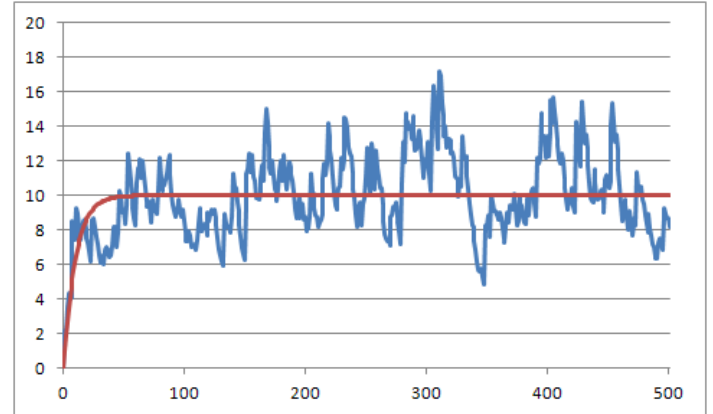

$\phi=0.9$

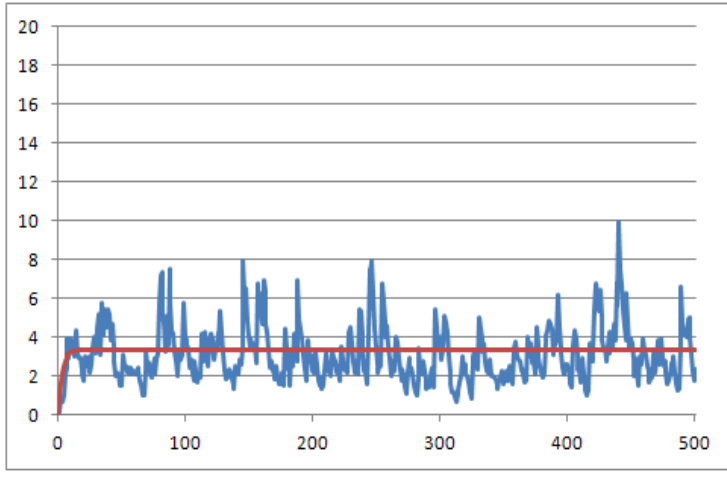

$\phi=0.7$

Figure 7.50 Representative output for $\operatorname{EAR(1)}$ for run length $n=500$ and batch size $b=1$, illustrating the dependency of the steady-state mean and rate of convergence on the parameter for $\phi=\{0.7,0.8,0.9,0.99\}$

Determining the "true" truncation point for this model is problematic. This is because the process converges to steady state only in the limit. Which observation to choose as the "true" truncation point is inherently subjective and open to second-guessing. In classical control engineering, the point where the response is "close enough" to steady state is called the settling time. The expected $\gamma$-percent settling time $\tau$ is implicitly defined by 


$$
\begin{aligned}
E\left[X_{\tau}\right] & =\frac{100-\gamma}{100} \phi E[\bar{X}]+1 \\
& =\frac{(100-\gamma) \phi}{100}\left(\frac{1}{1-\phi}\right)+1 \\
& =\frac{100-\gamma \phi}{100(1-\phi)}
\end{aligned}
$$

Common choices for the settling time are observations at which the responses are within $5 \%$ and $2 \%$ of their steady-state values. Table 7.24 provides settling times as a function of the parameter $\phi$ for a wide range of potential choices for $\gamma$.

Note that MSER is data driven and does not suffer from this ambiguity. On any replication, the process is deemed to have settled into steady state when the MSER statistic is minimized on the initial segment of observations. The expected settling time is approximately the average of these individual settling times over a very large number of replications. Note that this is the point argued by White and Hwang (2015) in response to Law (2015).

Table 7.24 Expected $\gamma$-percent settling time $\tau$ as a function of $\phi$ and $\gamma$

\begin{tabular}{lllll}
\hline & \multicolumn{5}{c}{$\phi$} \\
\cline { 2 - 5 }$\gamma$ & 0.7 & 0.8 & 0.9 & 0.99 \\
\hline 5 & 9 & 14 & 29 & 299 \\
\hline 2 & 11 & 18 & 38 & 390 \\
\hline 1 & 13 & 21 & 44 & 459 \\
\hline 0.1 & 20 & 31 & 66 & 688 \\
\hline 0.01 & 26 & 42 & 88 & 917 \\
\hline 0.001 & 33 & 52 & 110 & 1146 \\
\hline
\end{tabular}

In this chapter we explore the sensitivity of the estimated steady-state mean and truncation point with respect to the model parameter $\phi=\{0.7,0.8,0.9,0.99\}$ for batch sizes $b=\{1,5,10\}$ and eight different run lengths:

- $\quad$ long run lengths of $n=16000,8000,4000$ 
- medium run lengths of $n=2000,1000$

- $\quad$ short run lengths of $n=500,300,150,100$

We distinguish among these categories of run length experimentally, based on coverage of the mean by the truncated and/or raw $95 \%$ confidence intervals for $\phi=0.99$.

\subsubsection{Results for Model 3 with $\phi=0.99$}

Figure 7.51 shows the response of one of 1000 replications for $\phi=0.99$ and $n=16000$., together with the expected mean $E[\bar{X}]=100$.Visually, this output appears to settle into a steady-state operating regime after approximately 300-400 observations after which observations remain relatively stable about the mean. This corresponds to the traditional choice of a $2 \%$ to $5 \%$ settling time as applied control engineering.

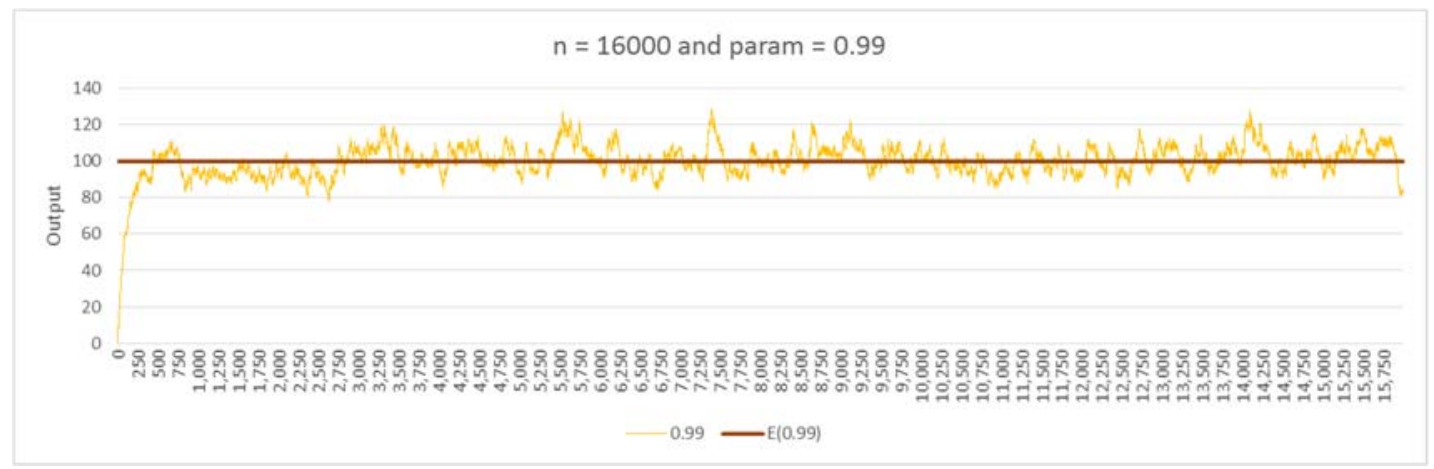

Figure 7.51 Example of Model 3 with $\phi=0.99(n=16000$, Brown line: $E(X))$

By halving the run length sequentially, the relationships among the variables—run length, batch size, and model parameter $\phi$ - and key metrics—means without truncation, truncated means, truncation points, the correlation between truncated means and truncation pointsbegin to emerge.

As can be seen in Figure 7.52, MSER yields superior estimates for all run lengths, with the difference in accuracy increasing as the run lengths as the decrease. For the long runs 
( $n=16000,8000,4000$ ), both the MSER-truncated and the raw confidence intervals cover the true mean. The comparatively large number of observations in steady state appears to be sufficient to overwhelm the initial transient. For medium-length runs $(n=2000,1000)$, only the MSER-truncated estimates provide coverage; for short-runs $(n=500,300,150$, 100), neither the MSER-truncated nor the raw confidence intervals cover the true mean. As the run length continues to decline, the ranges of truncated means become wider and estimation for every batch size increases.

This suggests that MSER needs to "see" at most 1000 observations on average in order to provide coverage for this system. As shown in Figure 7.53, this is approximately three times the length of the MSER-determined warm-up period. Batching is contraindicated below about $n=500$ with larger batches on average yielding smaller estimates and greater estimation error.

Larger batches are associated with greater truncation for every run length. The average MSER truncation point is reasonably consistent across large runs, on range $250<d^{*}<400$, as well as across medium-length runs, on the range $150<d^{*}<300$, and across short runs, on the range $0<d^{*}<200$. Restricting the optimal truncation point to $d^{*}<n / 2$ does not consistently appear to provide the desired indication that runs are too short to yield accurate estimates on average. However, the same is not true when the restriction is applied on a replicationby-replication basis, as recommended.

Figures 7.54 and Table 7.25 demonstrate that truncated mean estimates are relatively independent of the observations truncated. For long runs, the correlation is essentially zero. For medium and short runs, there appears to be a modest positive correlation, with the 
magnitude of this correlation generally increasing as run length decreases. As is perhaps intuitive, the shortest runs tend to induce greater truncation on average.
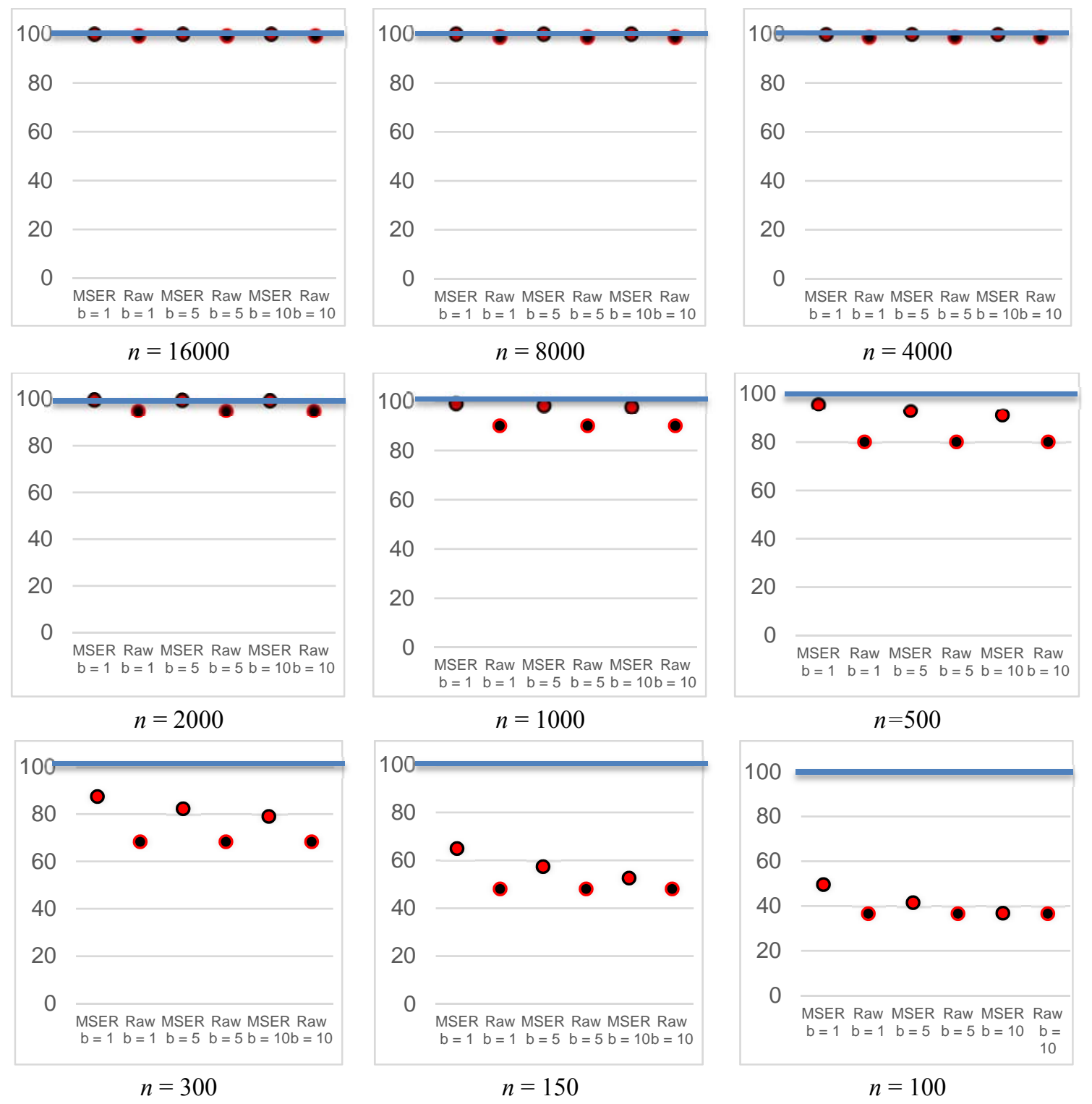

Figure $7.5295 \%$ confidence intervals of the mean for the truncated mean output as a function of batch size $(b=1,5$, and 10) and run length $(n=100,150,300,500,1000,2000,4000,8000$, and 16,000) for Model 3 with $\phi=0.99$ (Theoretical mean of 100, Blue line) 

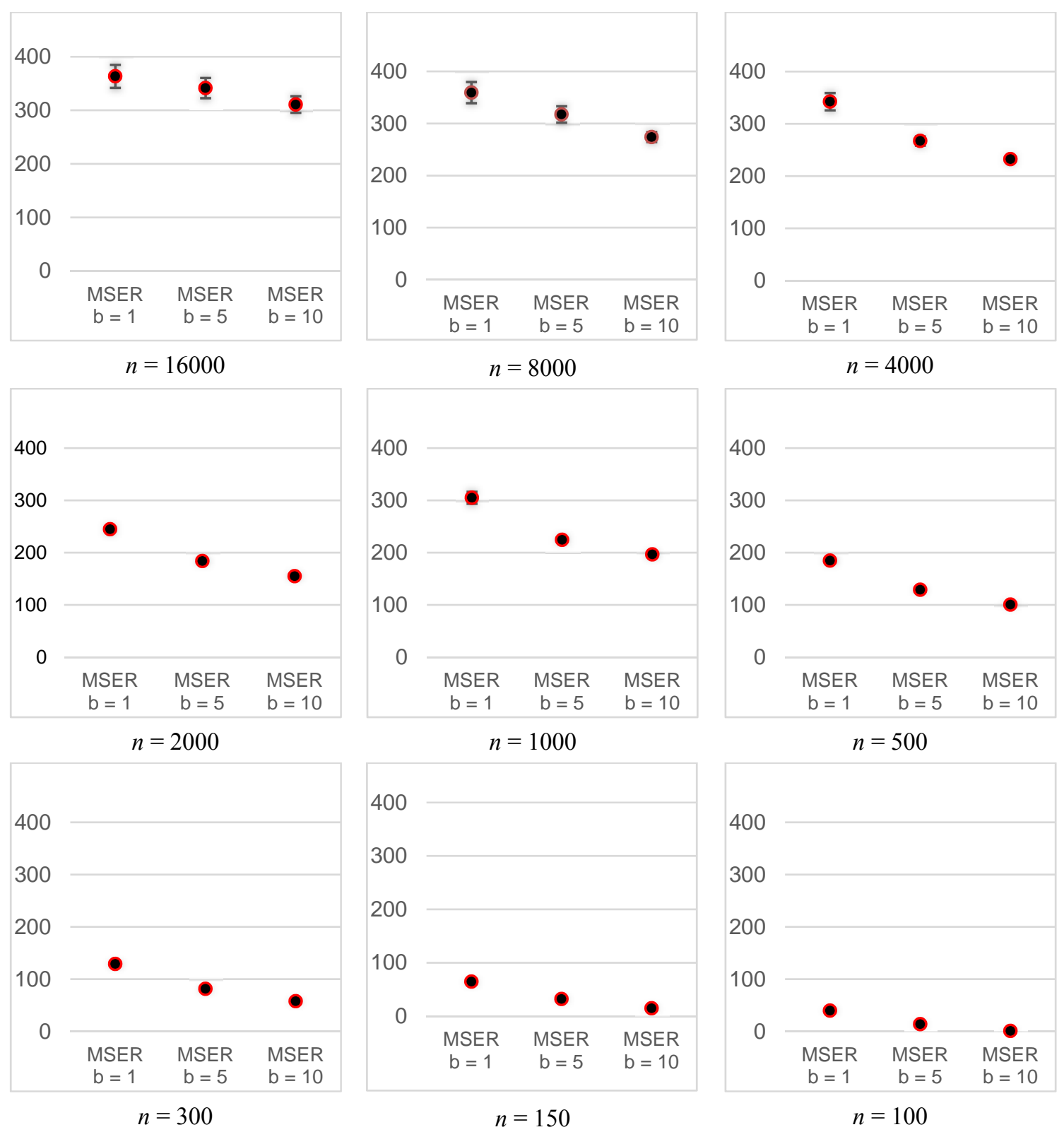

Figure $7.5395 \%$ confidence intervals for the mean number of observations truncated as a function of batch size $(b=1,5$, and 10) and run length $(n=100,150,300,500,1000,2000$, 4000,8000 , and 16,000) for Model 3 with $\phi=0.99$ 


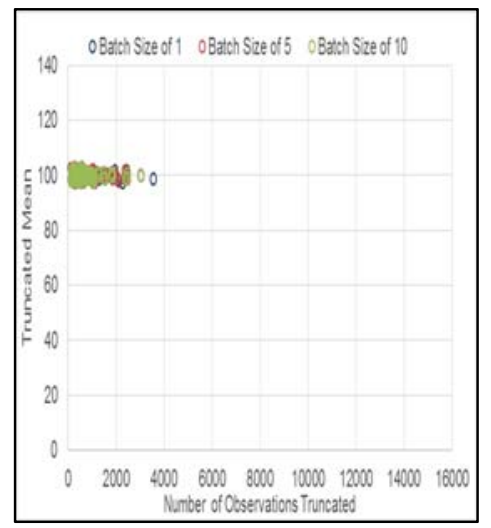

$n=16000$

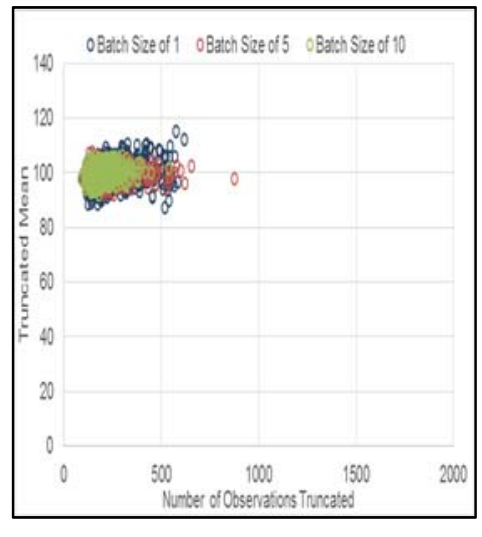

$n=2000$

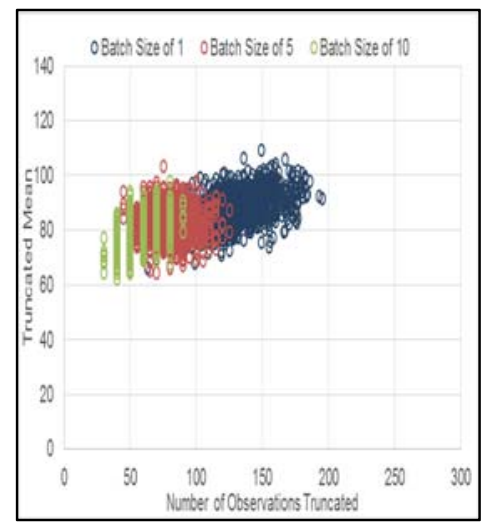

$n=300$

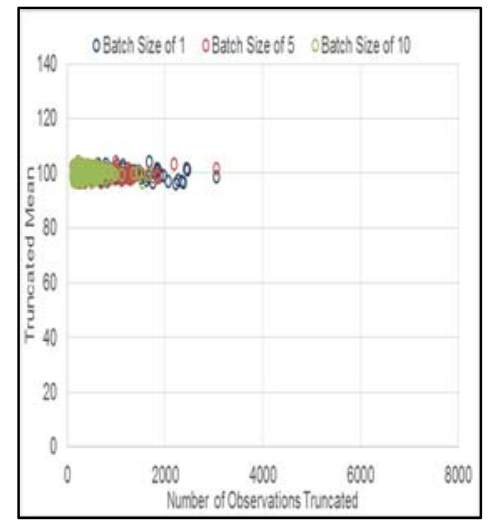

$n=8000$

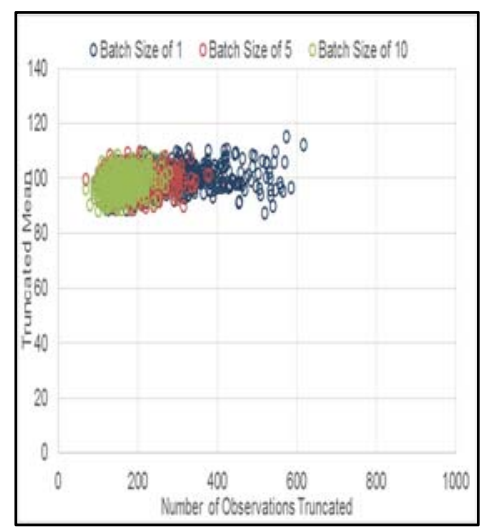

$n=1000$

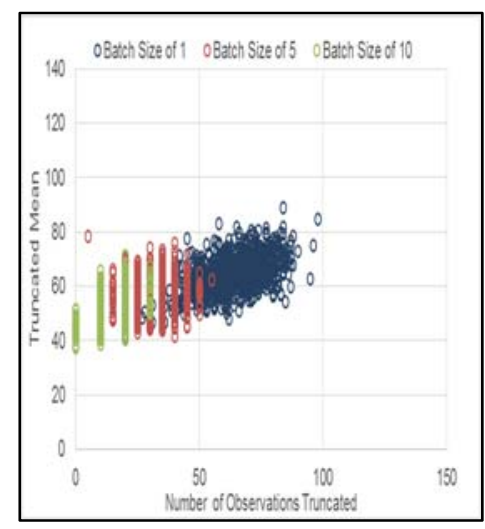

$n=150$

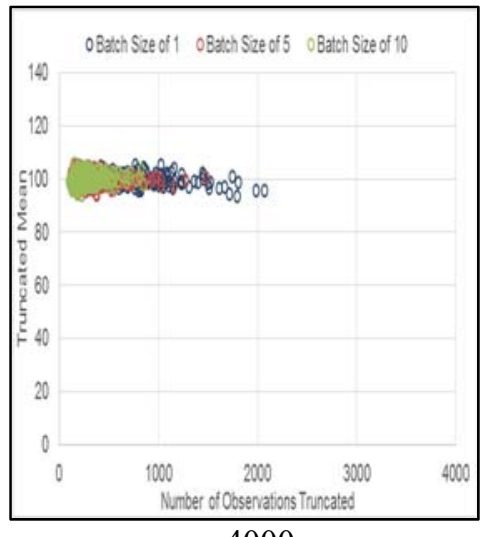

$n=4000$

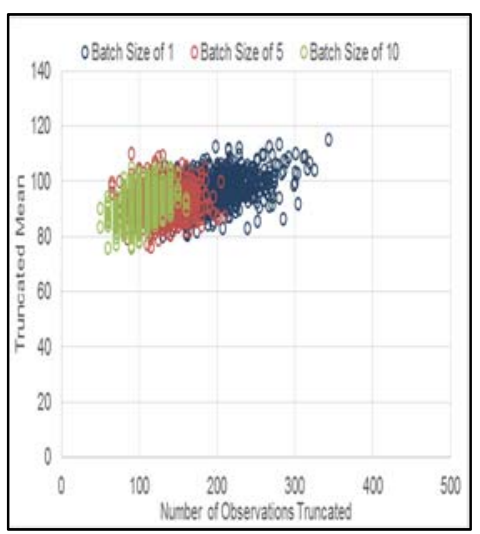

$n=500$

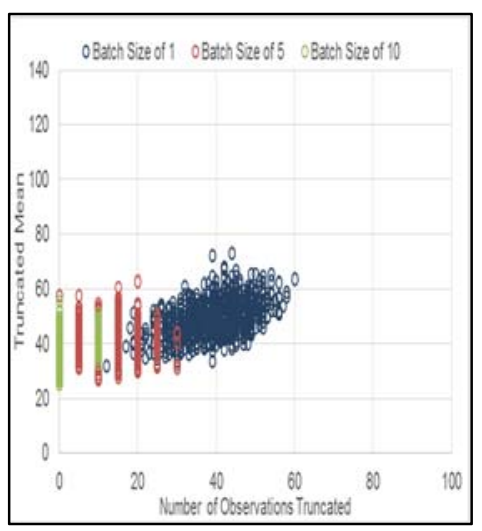

$n=100$

Figure 7.54 Scatterplots of the truncated mean vs. the number of observations truncated for batch sizes $b=1,5$, and 10 for run length of $n=100,150,300,500,1000,2000,4000,8000$, and 16,000 Model 3 with $\phi=0.99$ 
Table 7.25 Correlation between the truncated mean and the number of observations truncated for batch sizes $b=1,5$, and 10 for run length of $n=100,150,300,500,1000,2000,4000,8000$, and 16,000 for Model 3 with $\phi=0.99$

\begin{tabular}{|l|l|l|l|}
\hline & $\begin{array}{l}\text { Trunc } \\
\text { Batch } \\
=1\end{array}$ & $\begin{array}{l}\text { Trunc } \\
\text { Batch } \\
=5\end{array}$ & $\begin{array}{l}\text { Trunc } \\
\text { Batch } \\
=10\end{array}$ \\
\hline $\begin{array}{l}\text { Mean } \\
\text { Batch } \\
=1\end{array}$ & $\mathbf{- 0 . 0 5 2 7}$ & -0.0557 & -0.0163 \\
\hline $\begin{array}{l}\text { Mean } \\
\text { Batch } \\
=5\end{array}$ & -0.0424 & $\mathbf{- 0 . 0 5 5 8}$ & -0.0167 \\
\hline $\begin{array}{l}\text { Mean } \\
\text { Batch } \\
=10\end{array}$ & -0.0278 & -0.0393 & $\mathbf{- 0 . 0 1 5 5}$ \\
\hline
\end{tabular}

$$
n=16000
$$

\begin{tabular}{|l|l|l|l|}
\hline & $\begin{array}{l}\text { Trunc } \\
\text { Batch } \\
=1\end{array}$ & $\begin{array}{l}\text { Trunc } \\
\text { Batch } \\
=5\end{array}$ & $\begin{array}{l}\text { Trunc } \\
\text { Batch } \\
=10\end{array}$ \\
\hline $\begin{array}{l}\text { Mean } \\
\text { Batch } \\
=1\end{array}$ & $\mathbf{0 . 1 1 1 7}$ & 0.1870 & 0.1989 \\
\hline $\begin{array}{l}\text { Mean } \\
\text { Batch } \\
=5\end{array}$ & 0.1116 & $\mathbf{0 . 2 0 7 8}$ & 0.2194 \\
\hline $\begin{array}{l}\text { Mean } \\
\text { Batch } \\
=10\end{array}$ & 0.0812 & 0.1333 & $\mathbf{0 . 2 1 6 8}$ \\
\hline
\end{tabular}

$$
n=2000
$$

\begin{tabular}{|l|l|l|l|}
\hline & $\begin{array}{l}\text { Trunc } \\
\text { Batch } \\
=1\end{array}$ & $\begin{array}{l}\text { Trunc } \\
\text { Batch } \\
=5\end{array}$ & $\begin{array}{l}\text { Trunc } \\
\text { Batch } \\
=10\end{array}$ \\
\hline $\begin{array}{l}\text { Mean } \\
\text { Batch } \\
=1\end{array}$ & $\mathbf{0 . 5 0 4 0}$ & 0.2615 & 0.1539 \\
\hline $\begin{array}{l}\text { Mean } \\
\text { Batch } \\
=5\end{array}$ & 0.0708 & $\mathbf{0 . 4 4 4 4}$ & 0.2953 \\
\hline $\begin{array}{l}\text { Mean } \\
\text { Batch } \\
=10\end{array}$ & -0.0267 & 0.2004 & $\mathbf{0 . 3 9 0 6}$ \\
\hline
\end{tabular}

$$
n=300
$$

\begin{tabular}{|l|l|l|l|}
\hline & $\begin{array}{l}\text { Trunc } \\
\text { Batch } \\
=1\end{array}$ & $\begin{array}{l}\text { Trunc } \\
\text { Batch } \\
=5\end{array}$ & $\begin{array}{l}\text { Trunc } \\
\text { Batch } \\
=10\end{array}$ \\
\hline $\begin{array}{l}\text { Mean } \\
\text { Batch } \\
=1\end{array}$ & $\mathbf{- 0 . 0 7 3 5}$ & -0.0108 & 0.0453 \\
\hline $\begin{array}{l}\text { Mean } \\
\text { Batch } \\
=5\end{array}$ & -0.0427 & $\mathbf{- 0 . 0 1 7 5}$ & 0.0365 \\
\hline $\begin{array}{l}\text { Mean } \\
\text { Batch } \\
=10\end{array}$ & -0.0097 & 0.0248 & $\mathbf{0 . 0 4 2 9}$ \\
\hline
\end{tabular}

$$
n=8000
$$

\begin{tabular}{|l|l|l|l|}
\hline & $\begin{array}{l}\text { Trunc } \\
\text { Batch } \\
=1\end{array}$ & $\begin{array}{l}\text { Trunc } \\
\text { Batch } \\
=5\end{array}$ & $\begin{array}{l}\text { Trunc } \\
\text { Batch } \\
=10\end{array}$ \\
\hline $\begin{array}{l}\text { Mean } \\
\text { Batch } \\
=1\end{array}$ & $\mathbf{0 . 2 8 2 2}$ & 0.2843 & 0.2641 \\
\hline $\begin{array}{l}\text { Mean } \\
\text { Batch } \\
=5\end{array}$ & 0.1160 & $\mathbf{0 . 3 1 0 2}$ & 0.2861 \\
\hline $\begin{array}{l}\text { Mean } \\
\text { Batch } \\
=10\end{array}$ & 0.0348 & 0.1456 & $\mathbf{0 . 3 0 9 4}$ \\
\hline
\end{tabular}

$$
n=1000
$$

\begin{tabular}{|l|l|l|l|}
\hline & $\begin{array}{l}\text { Trunc } \\
\text { Batch } \\
=1\end{array}$ & $\begin{array}{l}\text { Trunc } \\
\text { Batch } \\
=5\end{array}$ & $\begin{array}{l}\text { Trunc } \\
\text { Batch } \\
=10\end{array}$ \\
\hline $\begin{array}{l}\text { Mean } \\
\text { Batch } \\
=1\end{array}$ & $\mathbf{0 . 4 7 7 1}$ & 0.1246 & -0.0443 \\
\hline $\begin{array}{l}\text { Mean } \\
\text { Batch } \\
=5\end{array}$ & 0.0104 & $\mathbf{0 . 4 4 5 5}$ & 0.2406 \\
\hline $\begin{array}{l}\text { Mean } \\
\text { Batch } \\
=10\end{array}$ & -0.1133 & 0.1392 & $\mathbf{0 . 4 7 4 8}$ \\
\hline
\end{tabular}

$$
n=150
$$

\begin{tabular}{|l|l|l|l|}
\hline & $\begin{array}{l}\text { Trunc } \\
\text { Batch } \\
=1\end{array}$ & $\begin{array}{l}\text { Trunc } \\
\text { Batch } \\
=5\end{array}$ & $\begin{array}{l}\text { Trunc } \\
\text { Batch } \\
=10\end{array}$ \\
\hline $\begin{array}{l}\text { Mean } \\
\text { Batch } \\
=1\end{array}$ & $\mathbf{- 0 . 0 4 5 8}$ & 0.1113 & 0.1575 \\
\hline $\begin{array}{l}\text { Mean } \\
\text { Batch } \\
=5\end{array}$ & 0.0149 & $\mathbf{0 . 1 1 5 7}$ & 0.1733 \\
\hline $\begin{array}{l}\text { Mean } \\
\text { Batch } \\
=10\end{array}$ & 0.0255 & 0.1242 & $\mathbf{0 . 1 6 8 7}$ \\
\hline
\end{tabular}

$$
n=4000
$$

\begin{tabular}{|l|l|l|l|}
\hline & $\begin{array}{l}\text { Trunc } \\
\text { Batch } \\
=1\end{array}$ & $\begin{array}{l}\text { Trunc } \\
\text { Batch } \\
=5\end{array}$ & $\begin{array}{l}\text { Trunc } \\
\text { Batch } \\
=10\end{array}$ \\
\hline $\begin{array}{l}\text { Mean } \\
\text { Batch } \\
=1\end{array}$ & $\mathbf{0 . 4 8 6 4}$ & 0.3049 & 0.2182 \\
\hline $\begin{array}{l}\text { Mean } \\
\text { Batch } \\
=5\end{array}$ & 0.1363 & $\mathbf{0 . 4 2 5 4}$ & 0.3383 \\
\hline $\begin{array}{l}\text { Mean } \\
\text { Batch } \\
=10\end{array}$ & 0.0348 & 0.2258 & $\mathbf{0 . 3 9 2 4}$ \\
\hline
\end{tabular}

$$
n=500
$$

\begin{tabular}{|l|l|l|l|}
\hline & $\begin{array}{l}\text { Trunc } \\
\text { Batch } \\
=1\end{array}$ & $\begin{array}{l}\text { Trunc } \\
\text { Batch } \\
=5\end{array}$ & $\begin{array}{l}\text { Trunc } \\
\text { Batch } \\
=10\end{array}$ \\
\hline $\begin{array}{l}\text { Mean } \\
\text { Batch } \\
=1\end{array}$ & $\mathbf{0 . 4 5 5 7}$ & 0.1240 & -0.0240 \\
\hline $\begin{array}{l}\text { Mean } \\
\text { Batch } \\
=5\end{array}$ & -0.0149 & $\mathbf{0 . 5 0 7 7}$ & 0.1365 \\
\hline $\begin{array}{l}\text { Mean } \\
\text { Batch } \\
=10\end{array}$ & -0.0693 & 0.1651 & $\mathbf{0 . 3 1 9 3}$ \\
\hline
\end{tabular}

$$
n=100
$$


-Batch Size of 1 - Batch Size of 5 - Batch Size of 10

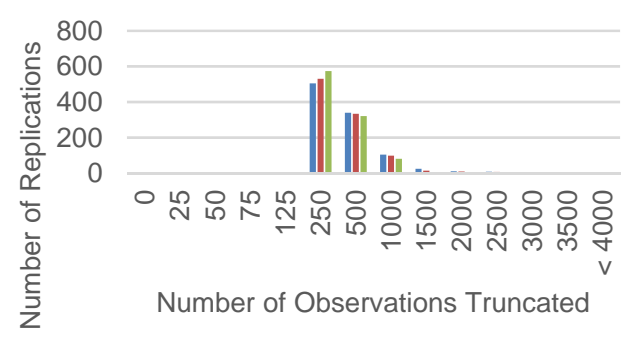

$$
n=16000
$$

match Size of 1 Batch Size of $5 \backsim$ Batch Size of 10

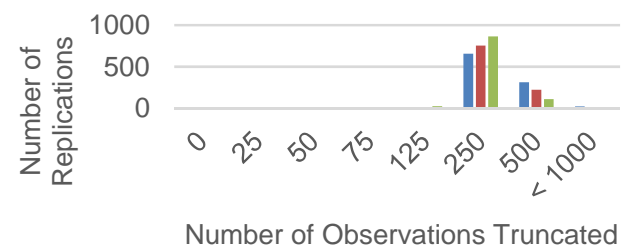

$$
n=2000
$$

घatch Size of $1 \backsim$ Batch Size of 5 Batch Size of 10

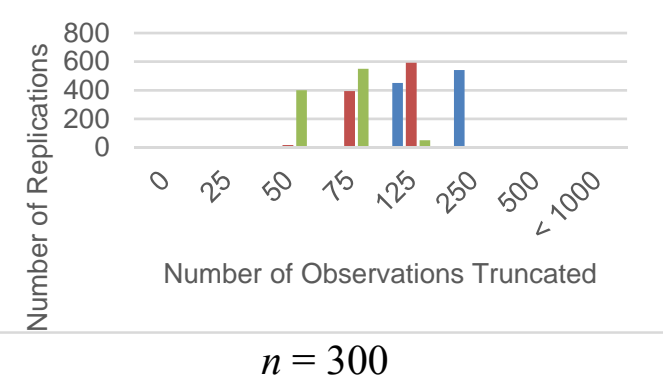

- Batch Size of 1 - Batch Size of 5 - Batch Size of 10

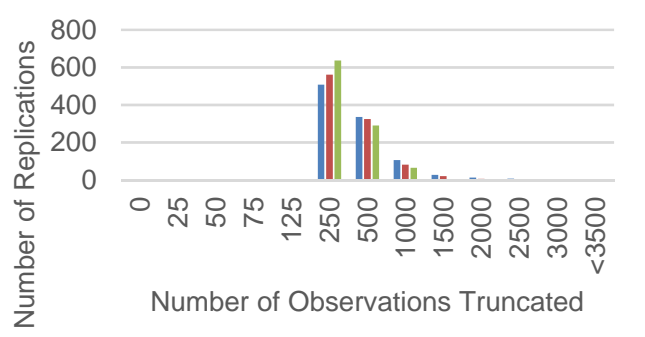

$$
n=8000
$$

Batch Size of 1 Batch Size of 5 Batch Size of 10

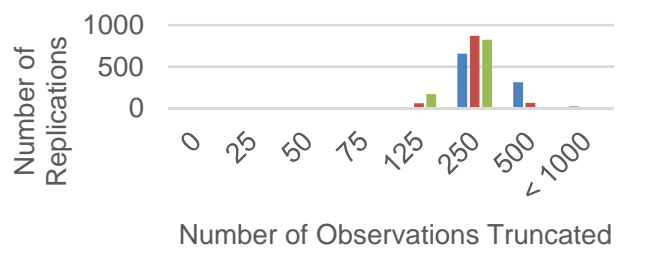

$$
n=1000
$$

- Batch Size of 1 - Batch Size of 5 Batch Size of 10

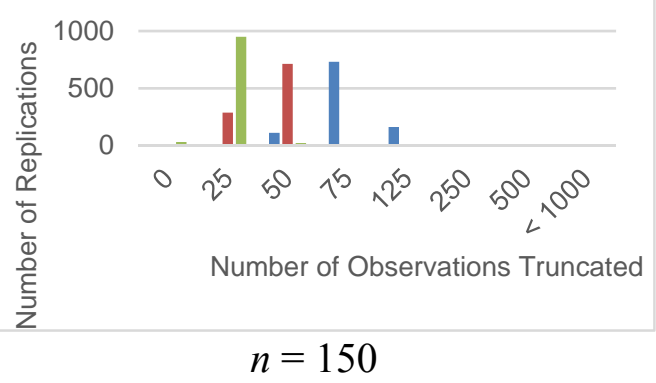

-Batch Size of 1 Batch Size of 5 Batch Size of 10

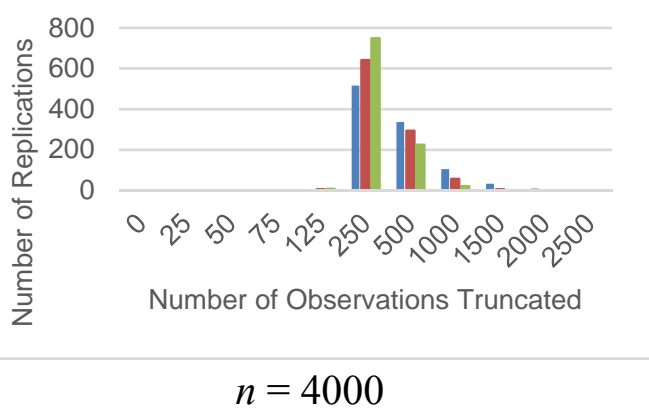

Batch Size of 1 Batch Size of $5 \square$ Batch Size of 10

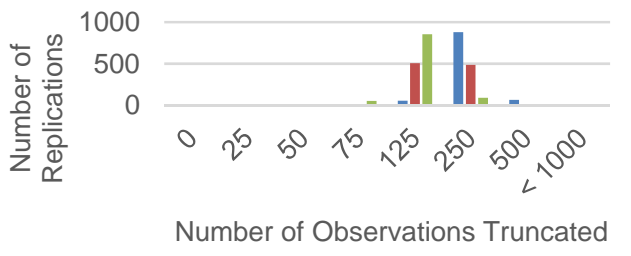

$$
n=500
$$

घatch Size of 1 घatch Size of 5 Batch Size of 10

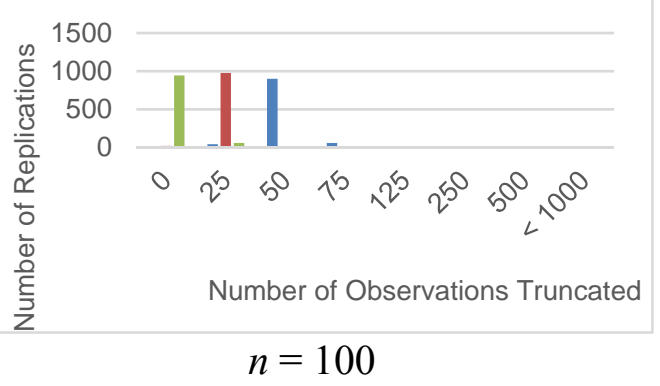

Figure 7.55 Frequency distribution of the number of observations truncated as a function of batch sizes $b=1,5$, and 10 for run length of $\mathrm{n}=100,150,300,500,1000,2000,4000,8000$, and 16,000 for Model 3 with of 0.99 


\subsubsection{Results for Model 3 with $\phi=0.90$}

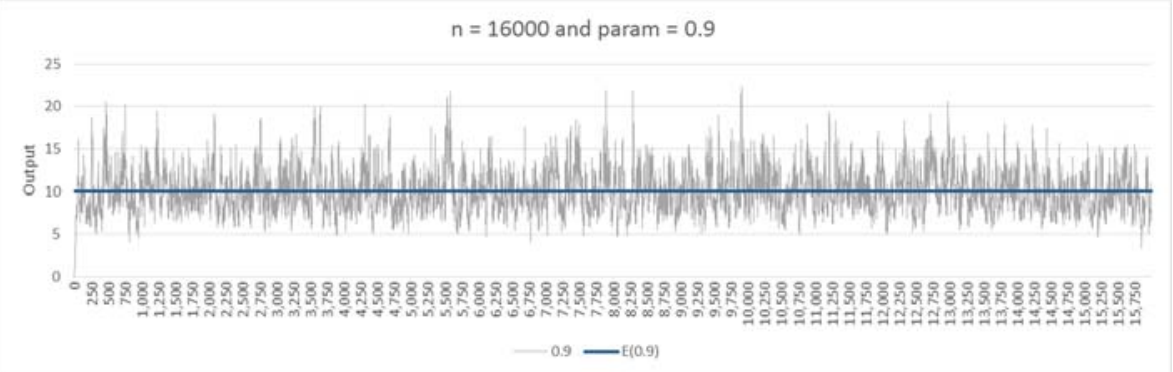

Figure 7.56 Example of EAR(1) with $\phi$ of $0.9(n=16000$, Blue line: $E(X))$
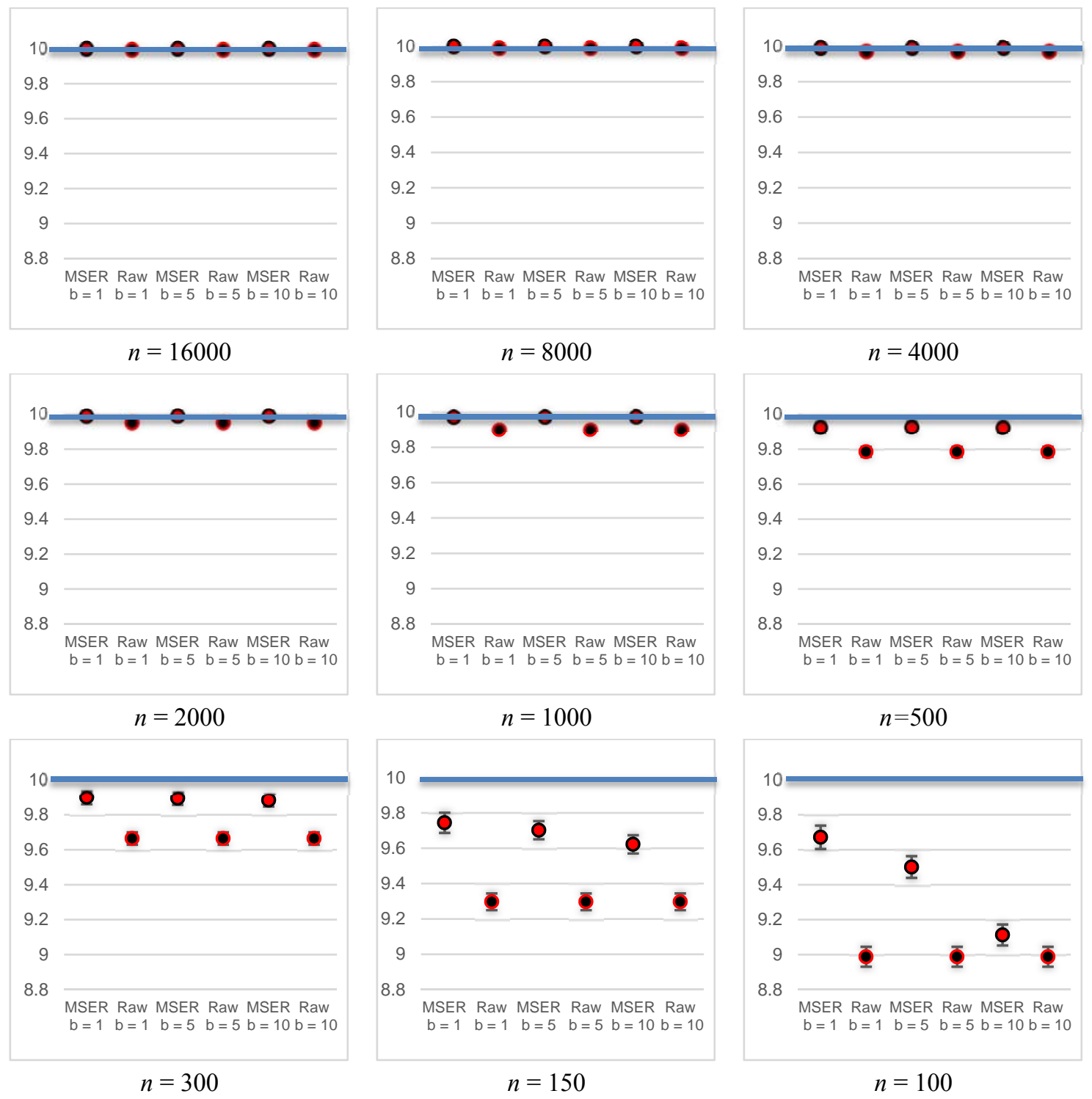

Figure $7.5795 \%$ confidence intervals of the mean for the truncated mean output as a function of batch size $(b=1,5$, and 10$)$ and run length $(n=100,150,300,500,1000,2000,4000,8000$, and 16,000) for Model 3 with $\phi=0.90$ (Theoretical mean of 10, Blue line) 

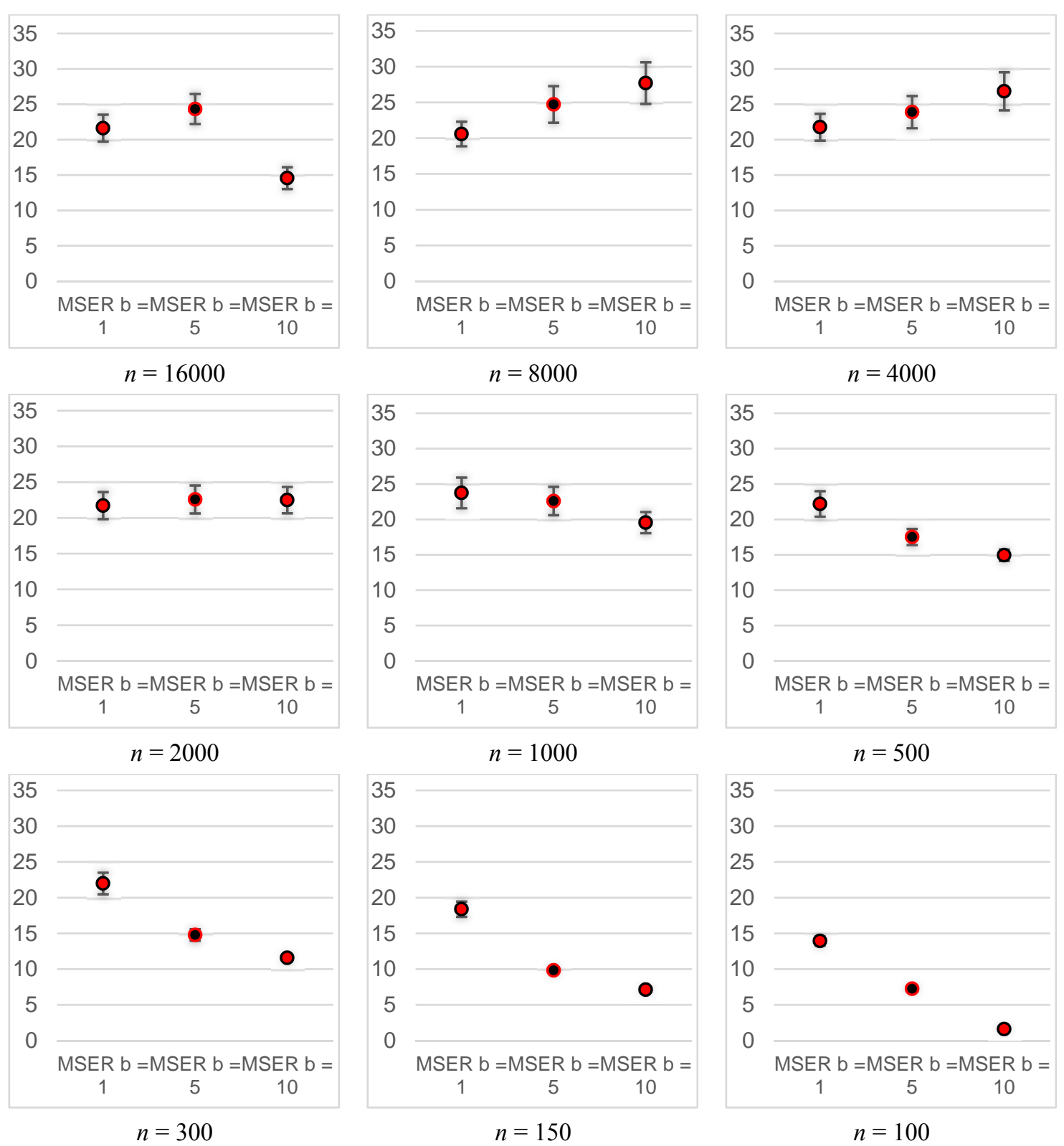

Figure $7.5895 \%$ confidence intervals for the mean number of observations truncated as a function of batch size $(b=1,5$, and 10) and run length $(n=100,150,300,500,1000,2000,4000$, 8000, and 16,000) for Model 3 with $\phi=0.90$ 

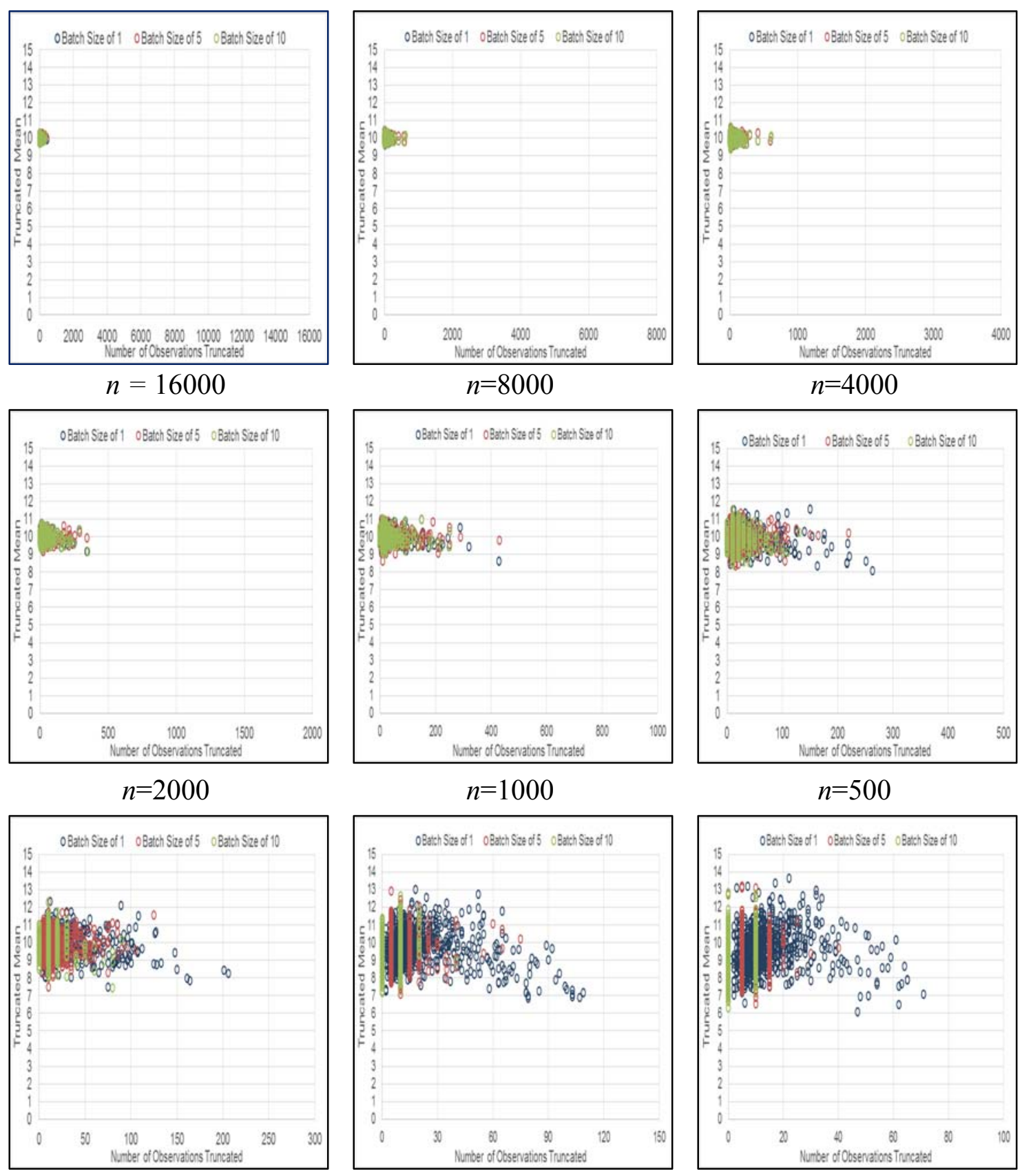

$$
n=300
$$

$$
n=150
$$

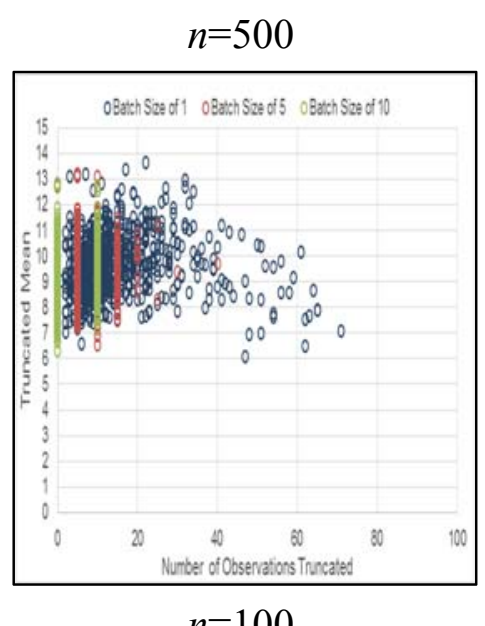

Figure 7.59 Scatterplots of the truncated mean vs. the number of observations truncated for batch sizes $b=1,5$, and 10 for run length of $n=100,150,300,500,1000,2000,4000,8000$, and 16,000 Model 3 with $\phi=0.90$ 
Table 7.26 Correlation between the truncated mean and the number of observations truncated for batch sizes $b=1,5$, and 10 for run length of $n=100,150,300,500,1000,2000$, 4000, 8000, and 16,000 for Model 3 with $\phi=0.90$

\begin{tabular}{|l|l|l|l|}
\hline & $\begin{array}{l}\text { Trunc } \\
\text { Batch } \\
=1\end{array}$ & $\begin{array}{l}\text { Trunc } \\
\text { Batch } \\
=5\end{array}$ & $\begin{array}{l}\text { Trunc } \\
\text { Batch } \\
=10\end{array}$ \\
\hline $\begin{array}{l}\text { Mean } \\
\text { Batch } \\
=1\end{array}$ & $\mathbf{0 . 0 0 9 4}$ & -0.0087 & 0.0276 \\
\hline $\begin{array}{l}\text { Mean } \\
\text { Batch } \\
=5\end{array}$ & 0.0089 & $\mathbf{- 0 . 0 1 1 1}$ & 0.0261 \\
\hline $\begin{array}{l}\text { Mean } \\
\text { Batch } \\
=10\end{array}$ & 0.0076 & -0.0119 & $\mathbf{0 . 0 1 9 8}$ \\
\hline
\end{tabular}

\begin{tabular}{|l|l|l|l|}
\hline & $\begin{array}{l}\text { Trunc } \\
\text { Batch } \\
=1\end{array}$ & $\begin{array}{l}\text { Trunc } \\
\text { Batch =5 }\end{array}$ & $\begin{array}{l}\text { Trunc } \\
\text { Batch } \\
=10\end{array}$ \\
\hline $\begin{array}{l}\text { Mean } \\
\text { Batch } \\
=1\end{array}$ & $\mathbf{- 0 . 0 3 2 9}$ & -0.0314 & 0.0107 \\
\hline $\begin{array}{l}\text { Mean } \\
\text { Batch } \\
=5\end{array}$ & -0.0328 & $\mathbf{- 0 . 0 4 6 7}$ & -0.0026 \\
\hline $\begin{array}{l}\text { Mean } \\
\text { Batch } \\
=10\end{array}$ & -0.0327 & -0.0468 & $\mathbf{- 0 . 0 0 3 2}$ \\
\hline
\end{tabular}

$n=16000$

\begin{tabular}{|l|l|l|l|}
\hline & $\begin{array}{l}\text { Trunc } \\
\text { Batch } \\
=1\end{array}$ & $\begin{array}{l}\text { Trunc } \\
\text { Batch } \\
=5\end{array}$ & $\begin{array}{l}\text { Trunc } \\
\text { Batch } \\
=10\end{array}$ \\
\hline $\begin{array}{l}\text { Mean } \\
\text { Batch } \\
=1\end{array}$ & $\mathbf{- 0 . 1 2 4 9}$ & -0.1247 & -0.1125 \\
\hline $\begin{array}{l}\text { Mean } \\
\text { Batch } \\
=5\end{array}$ & -0.1251 & $\mathbf{- 0 . 1 2 2 0}$ & -0.1107 \\
\hline $\begin{array}{l}\text { Mean } \\
\text { Batch } \\
=10\end{array}$ & -0.1152 & -0.1134 & $\mathbf{- 0 . 1 1 2 1}$ \\
\hline
\end{tabular}

$n=2000$

\begin{tabular}{|l|l|l|l|}
\multicolumn{1}{|c|}{$n=8000$} \\
\hline & $\begin{array}{l}\text { Trunc } \\
\text { Batch } \\
=1\end{array}$ & $\begin{array}{l}\text { Trunc } \\
\text { Batch } \\
=5\end{array}$ & $\begin{array}{l}\text { Trunc } \\
\text { Batch } \\
=10\end{array}$ \\
\hline $\begin{array}{l}\text { Mean } \\
\text { Batch }= \\
1\end{array}$ & $\mathbf{- 0 . 1 1 9 4}$ & -0.1071 & -0.0355 \\
\hline $\begin{array}{l}\text { Mean } \\
\text { Batch }= \\
5\end{array}$ & -0.1025 & $\mathbf{- 0 . 1 0 4 4}$ & -0.0354 \\
\hline $\begin{array}{l}\text { Mean } \\
\text { Batch }= \\
10\end{array}$ & -0.0457 & -0.0442 & $\mathbf{- 0 . 0 3 4 4}$ \\
\hline
\end{tabular}

\begin{tabular}{|l|l|l|l|}
\hline & $\begin{array}{l}\text { Trunc } \\
\text { Batch } \\
=1\end{array}$ & $\begin{array}{l}\text { Trunc } \\
\text { Batch } \\
=5\end{array}$ & $\begin{array}{l}\text { Trunc } \\
\text { Batch } \\
=10\end{array}$ \\
\hline $\begin{array}{l}\text { Mean } \\
\text { Batch }= \\
1\end{array}$ & $\mathbf{- 0 . 0 2 7 7}$ & -0.0166 & 0.0038 \\
\hline $\begin{array}{l}\text { Mean } \\
\text { Batch } \\
=5\end{array}$ & -0.0267 & $\mathbf{- 0 . 0 3 4 7}$ & -0.0110 \\
\hline $\begin{array}{l}\text { Mean } \\
\text { Batch } \\
=10\end{array}$ & -0.0263 & -0.0337 & $\mathbf{- 0 . 0 1 4 9}$ \\
\hline
\end{tabular}

$n=4000$

\begin{tabular}{|l|l|l|l|}
\hline & $\begin{array}{l}\text { Trunc } \\
\text { Batch } \\
=1\end{array}$ & $\begin{array}{l}\text { Trunc } \\
\text { Batch } \\
=5\end{array}$ & $\begin{array}{l}\text { Trunc } \\
\text { Batch } \\
=10\end{array}$ \\
\hline $\begin{array}{l}\text { Mean } \\
\text { Batch }= \\
1\end{array}$ & $\mathbf{- 0 . 1 6 0 9}$ & -0.0987 & -0.0565 \\
\hline $\begin{array}{l}\text { Mean } \\
\text { Batch } \\
=5\end{array}$ & -0.0776 & $\mathbf{- 0 . 0 9 4 5}$ & -0.0507 \\
\hline $\begin{array}{l}\text { Mean } \\
\text { Batch } \\
=10\end{array}$ & -0.0460 & -0.0417 & $\mathbf{- 0 . 0 4 1 8}$ \\
\hline
\end{tabular}

\begin{tabular}{|l|l|l|l|}
\hline & $\begin{array}{l}\text { Trunc } \\
\text { Batch } \\
=1\end{array}$ & $\begin{array}{l}\text { Trunc } \\
\text { Batch } \\
=5\end{array}$ & $\begin{array}{l}\text { Trunc } \\
\text { Batch } \\
=10\end{array}$ \\
\hline $\begin{array}{l}\text { Mean } \\
\text { Batch } \\
=1\end{array}$ & $\mathbf{- 0 . 1 2 1 3}$ & -0.0715 & -0.0633 \\
\hline $\begin{array}{l}\text { Mean } \\
\text { Batch } \\
=5\end{array}$ & -0.0001 & $\mathbf{- 0 . 0 5 2 8}$ & -0.0731 \\
\hline $\begin{array}{l}\text { Mean } \\
\text { Batch } \\
=10\end{array}$ & 0.0172 & -0.0193 & $\mathbf{- 0 . 0 5 5 8}$ \\
\hline
\end{tabular}

\begin{tabular}{|l|l|l|l|}
\hline & $\begin{array}{l}\text { Trunc } \\
\text { Batch } \\
=1\end{array}$ & $\begin{array}{l}\text { Trunc } \\
\text { Batch } \\
=5\end{array}$ & $\begin{array}{l}\text { Trunc } \\
\text { Batch } \\
=10\end{array}$ \\
\hline $\begin{array}{l}\text { Mean } \\
\text { Batch }= \\
1\end{array}$ & $\mathbf{- 0 . 1 7 7 6}$ & 0.0518 & 0.2247 \\
\hline $\begin{array}{l}\text { Mean } \\
\text { Batch }= \\
5\end{array}$ & 0.0080 & $\mathbf{0 . 0 6 4 0}$ & 0.2239 \\
\hline $\begin{array}{l}\text { Mean } \\
\text { Batch }= \\
10\end{array}$ & 0.0299 & 0.1226 & $\mathbf{0 . 3 2 0 3}$ \\
\hline
\end{tabular}

$n=150$

\begin{tabular}{|l|l|l|l|}
\hline & $\begin{array}{l}\text { Trunc } \\
\text { Batch } \\
=1\end{array}$ & $\begin{array}{l}\text { Trunc } \\
\text { Batch } \\
=5\end{array}$ & $\begin{array}{l}\text { Trunc } \\
\text { Batch } \\
=10\end{array}$ \\
\hline $\begin{array}{l}\text { Mean } \\
\text { Batch }= \\
1\end{array}$ & $\mathbf{0 . 0 3 5 6}$ & 0.1800 & 0.1167 \\
\hline $\begin{array}{l}\text { Mean } \\
\text { Batch } \\
=5\end{array}$ & 0.1101 & $\mathbf{0 . 2 0 3 4}$ & 0.1250 \\
\hline $\begin{array}{l}\text { Mean } \\
\text { Batch } \\
=10\end{array}$ & 0.0835 & 0.1562 & $\mathbf{0 . 2 7 0 2}$ \\
\hline
\end{tabular}

$n=100$ 


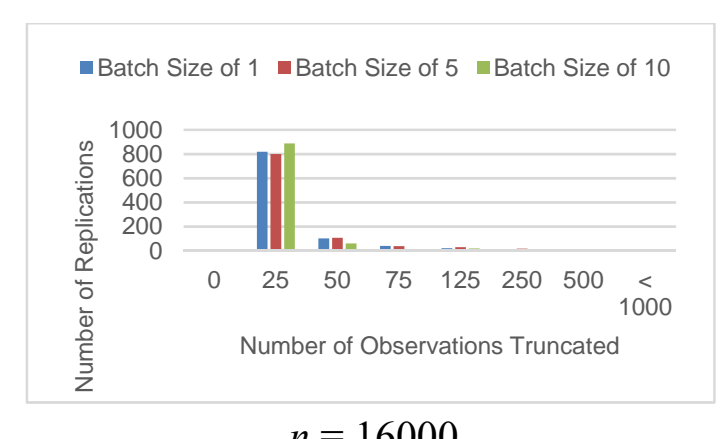

$$
n=16000
$$

\#atch Size of 1 Batch Size of 5 Batch Size of 10

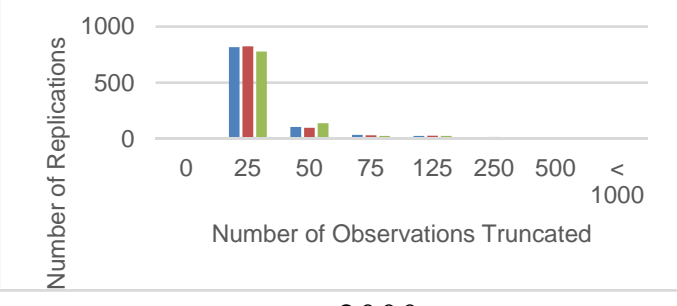

$$
n=2000
$$

match Size of 1 Batch Size of 5 Batch Size of 10

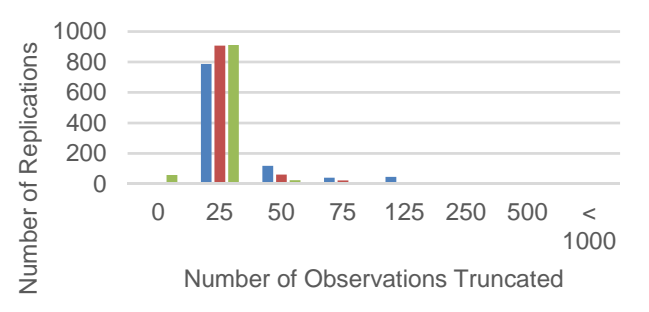

$$
n=300
$$

Figure 7.60 Frequency distribution of the number of observations truncated as a function of batch sizes $b=1,5$, and 10 for run length of $n=100,150,300,500,1000,2000,4000,8000$, and 16,000 for Model 3 with $\phi$ of 0.90
Batch Size of 1 Batch Size of 5 -Batch Size of 10

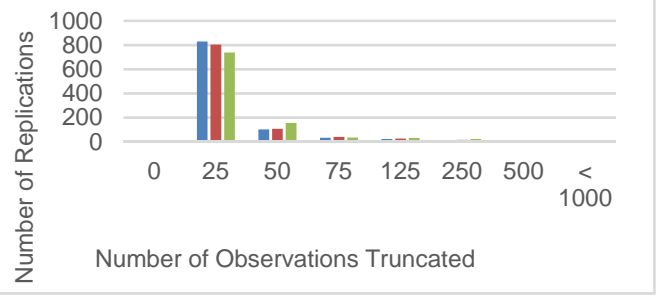

$$
n=8000
$$

घatch Size of 1 Batch Size of 5 Batch Size of 10

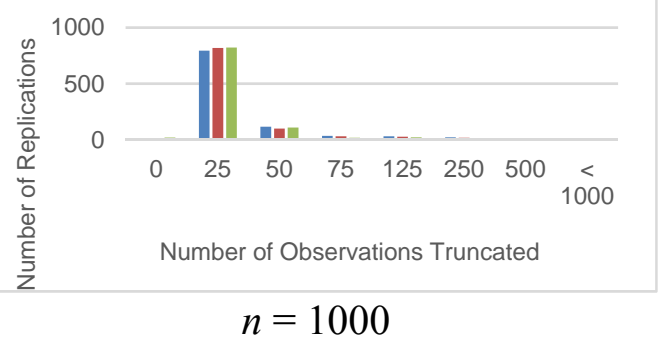

match Size of 1 Batch Size of 5 Batch Size of 10

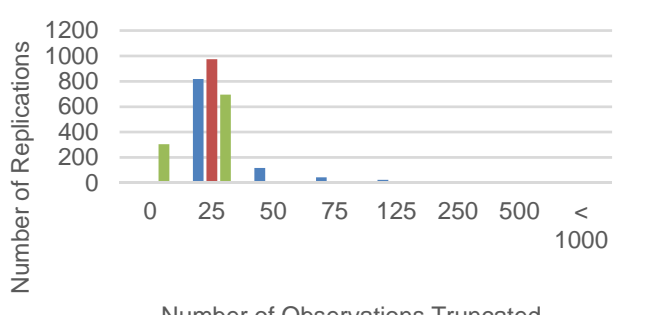

Number of Observations Truncated

$$
n=150
$$

-Batch Size of 1 -Batch Size of 5 Batch Size of 10

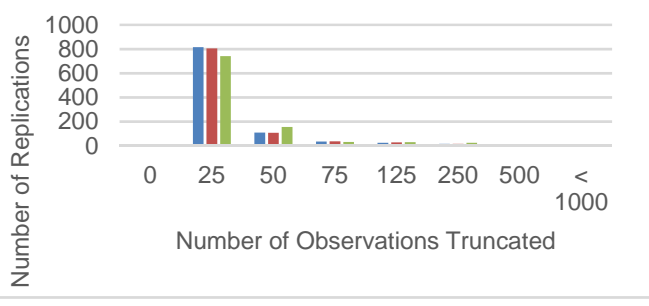

$$
n=4000
$$

-Batch Size of 1 Batch Size of $5 \square$ Batch Size of 10

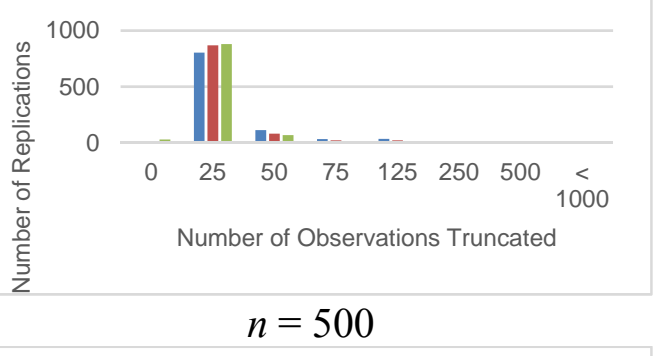

-Batch Size of 1 Batch Size of 5 Batch Size of 10

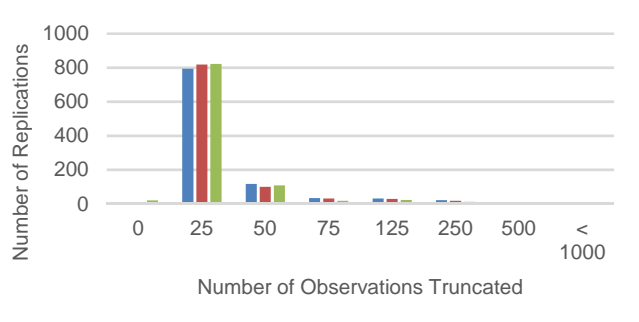

$$
n=100
$$




\subsubsection{Results for Model 3 with $\phi=0.80$}

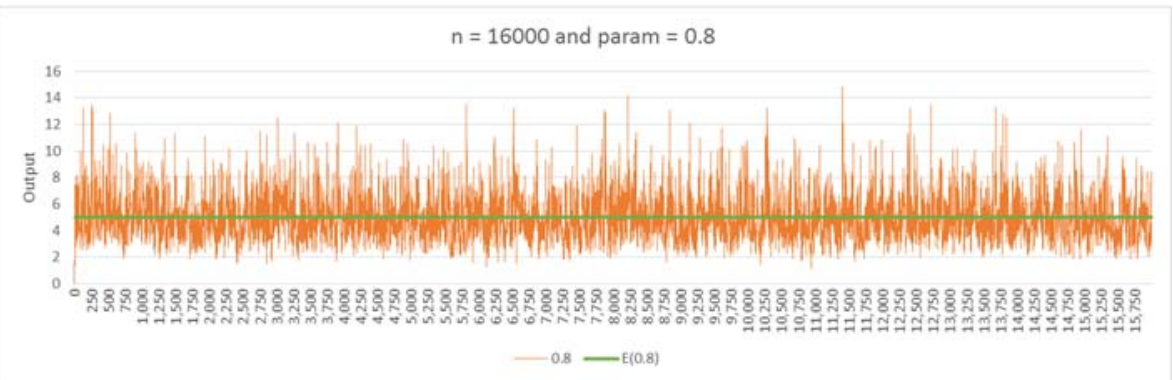

Figure 7.61 Example of EAR(1) with $\phi$ of $0.8(n=16000$, Green line: $\mathrm{E}(X))$
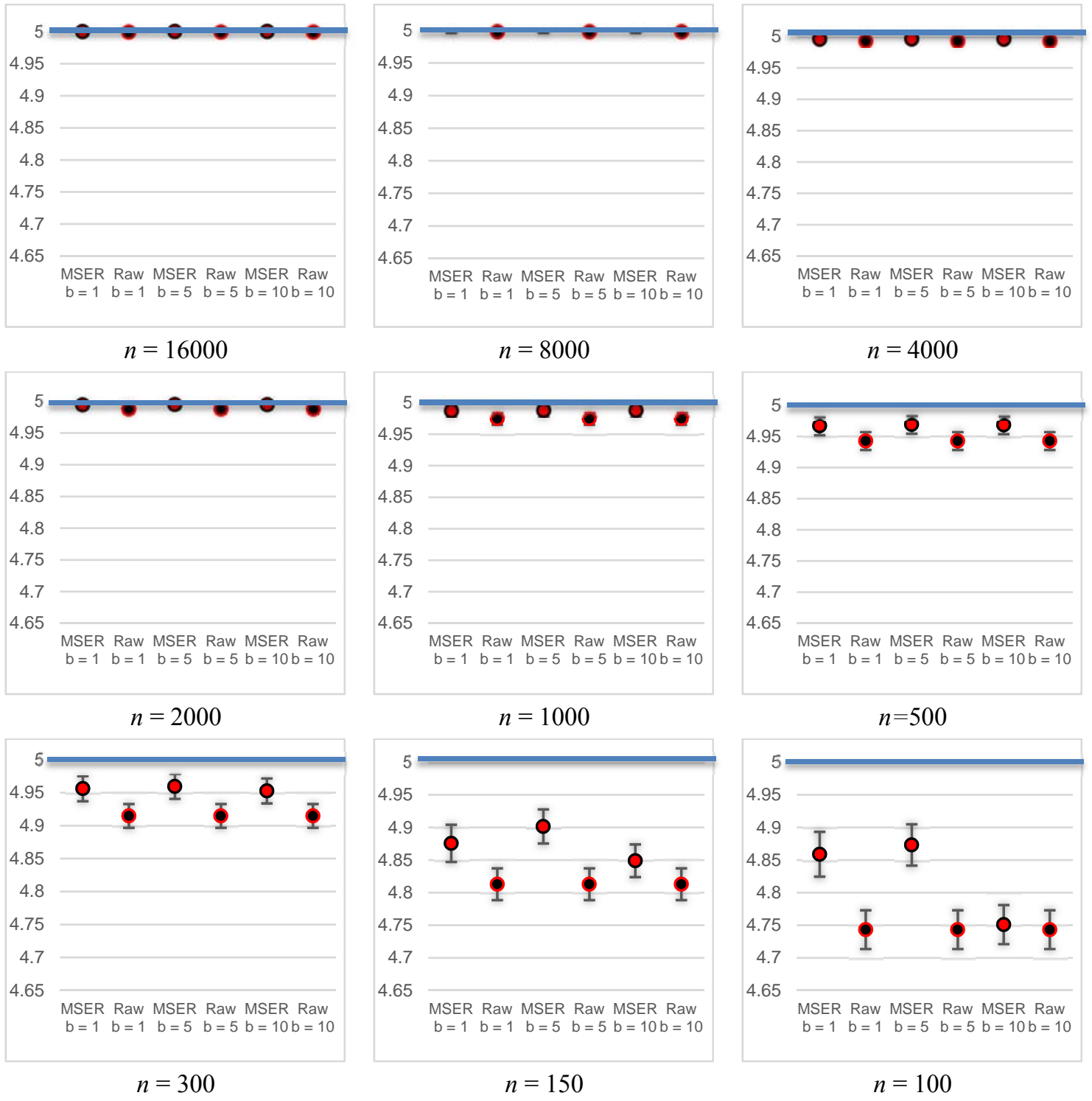

Figure $7.6295 \%$ confidence intervals of the mean for the truncated mean output as a function of batch size $(b=1,5$, and 10) and run length $(n=100,150,300,500,1000,2000,4000,8000$, and 16,000) for Model 3 with $\phi=0.80$ (Theoretical mean of 5 , Blue line) 

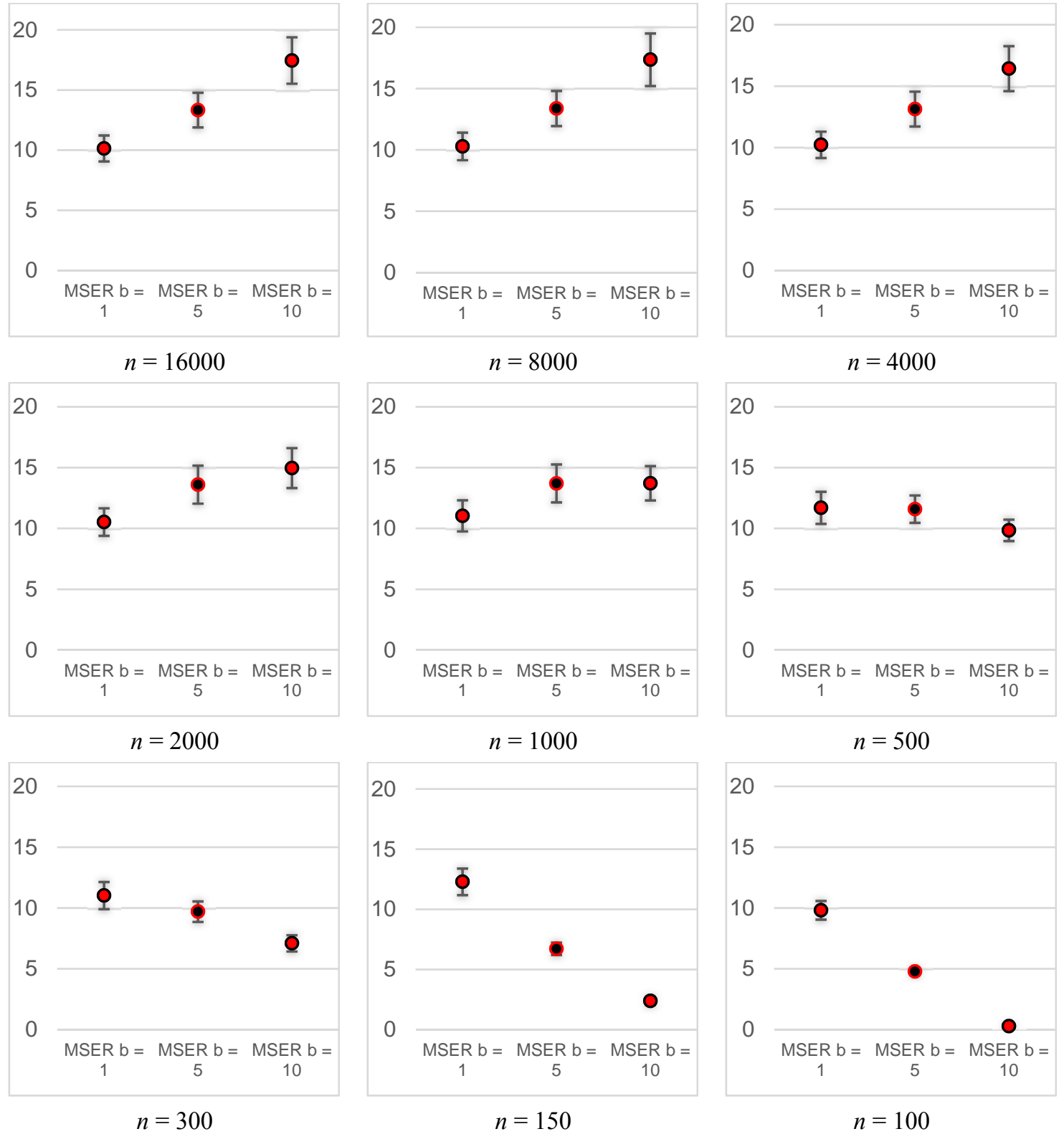

Figure 7.63 95\% confidence intervals for the mean number of observations truncated as a function of batch size $(b=1,5$, and 10) and run length $(n=100,150,300,500,1000,2000,4000$, 8000, and 16,000) for Model 3 with $\phi=0.80$ 


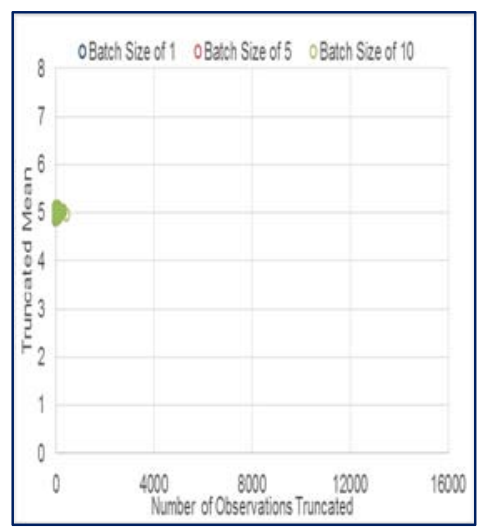

$n=16000$

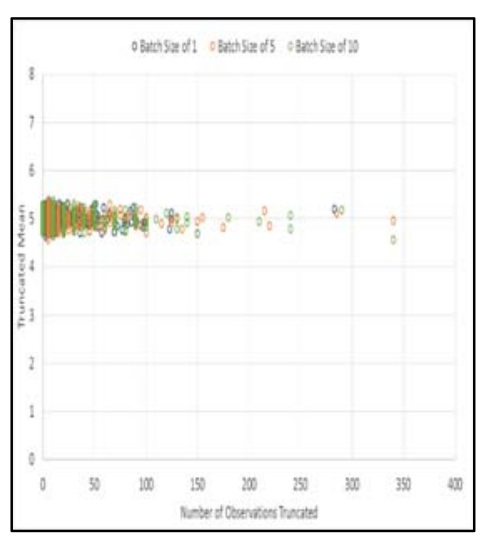

$n=2000$

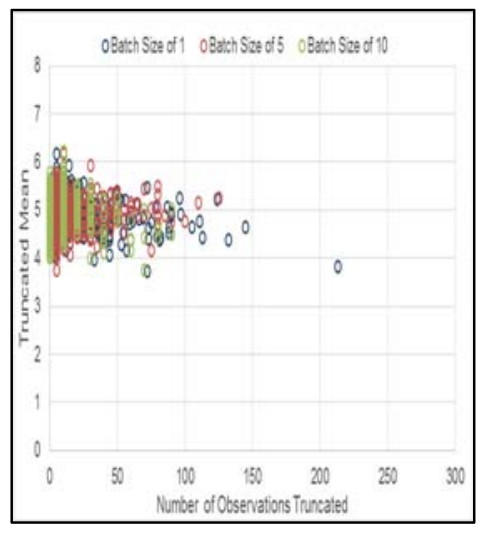

$n=300$

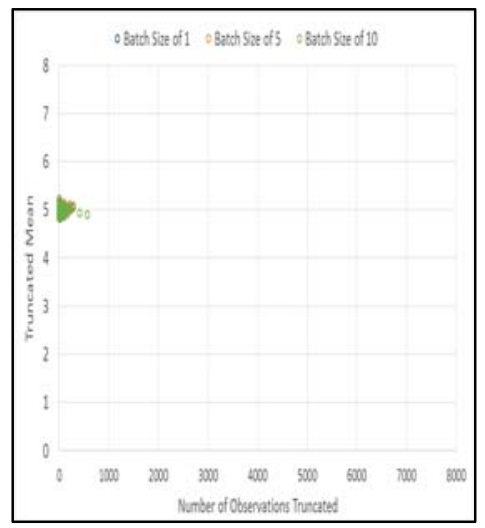

$n=8000$

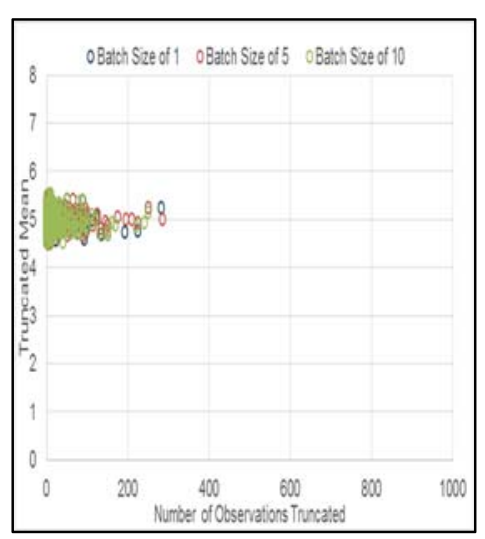

$n=1000$

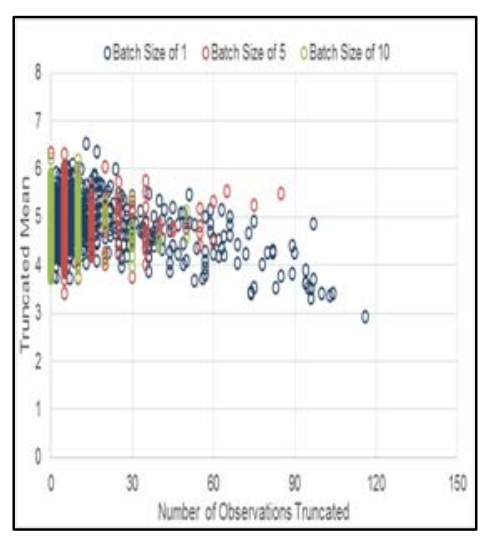

$n=150$

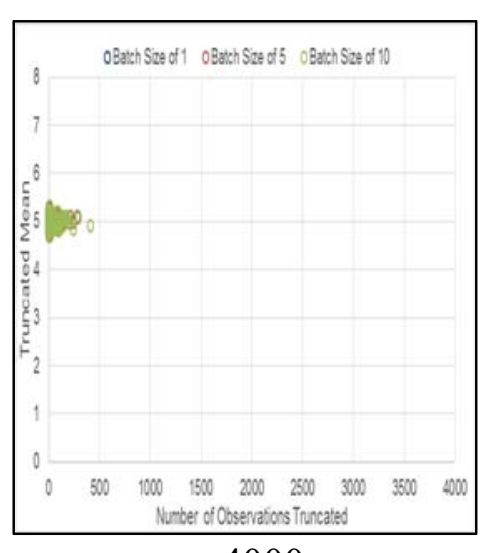

$n=4000$

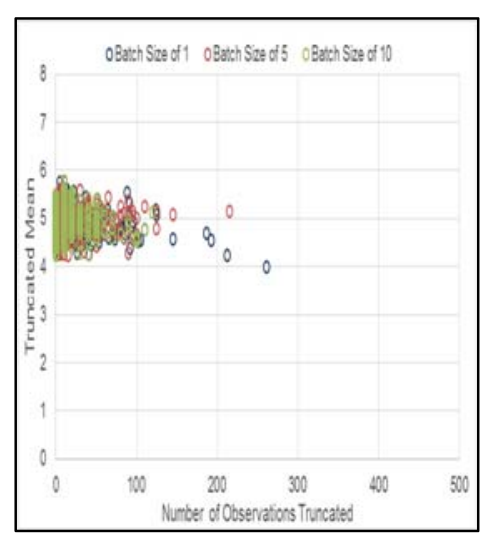

$n=500$

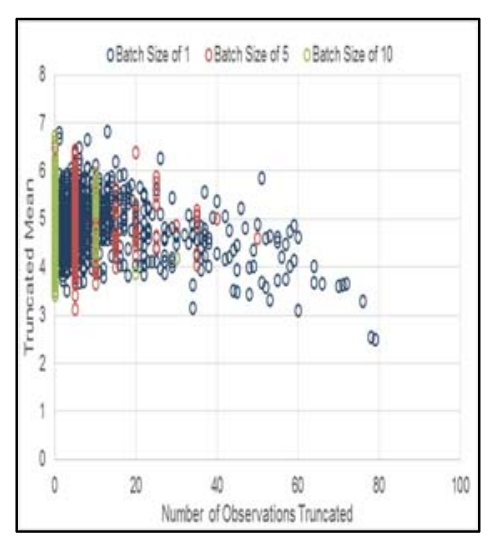

$n=100$

Figure 7.64 Scatterplots of the truncated mean vs. the number of observations truncated for batch sizes $b=1,5$, and 10 for run length of $n=100,150,300,500,1000,2000,4000,8000$, and 16,000 Model 3 with $\phi=0.80$ 
Table 7.27 Correlation between the truncated mean and the number of observations truncated for batch sizes $b=1,5$, and 10 for run length of $n=100,150,300,500,1000,2000$, 4000, 8000, and 16,000 for Model 3 with $\phi=0.80$

\begin{tabular}{|l|l|l|l|}
\hline & $\begin{array}{l}\text { Trunc } \\
\text { Batch } \\
=1\end{array}$ & $\begin{array}{l}\text { Trunc } \\
\text { Batch } \\
=5\end{array}$ & $\begin{array}{l}\text { Trunc } \\
\text { Batch } \\
=10\end{array}$ \\
\hline $\begin{array}{l}\text { Mean } \\
\text { Batch } \\
=1\end{array}$ & $\mathbf{0 . 0 5 1 9}$ & 0.0491 & 0.0431 \\
\hline $\begin{array}{l}\text { Mean } \\
\text { Batch } \\
=5\end{array}$ & 0.0508 & $\mathbf{0 . 0 4 1 9}$ & 0.0387 \\
\hline $\begin{array}{l}\text { Mean } \\
\text { Batch } \\
=10\end{array}$ & 0.0509 & 0.0437 & $\mathbf{0 . 0 3 3 8}$ \\
\hline
\end{tabular}

$n=16000$

\begin{tabular}{|l|l|l|l|}
\hline & $\begin{array}{l}\text { Trunc } \\
\text { Batch } \\
=1\end{array}$ & $\begin{array}{l}\text { Trunc } \\
\text { Batch } \\
=5\end{array}$ & $\begin{array}{l}\text { Trunc } \\
\text { Batch } \\
=10\end{array}$ \\
\hline $\begin{array}{l}\text { Mean } \\
\text { Batch } \\
=1\end{array}$ & $\mathbf{- 0 . 0 2 6 4}$ & -0.0447 & -0.0443 \\
\hline $\begin{array}{l}\text { Mean } \\
\text { Batch } \\
=5\end{array}$ & -0.0302 & $\mathbf{- 0 . 0 7 9 9}$ & -0.0725 \\
\hline $\begin{array}{l}\text { Mean } \\
\text { Batch } \\
=10\end{array}$ & -0.0272 & -0.0725 & $\mathbf{- 0 . 0 7 3 6}$ \\
\hline
\end{tabular}

$n=2000$

\begin{tabular}{|l|l|l|l|}
\hline & $\begin{array}{l}\text { Trunc } \\
\text { Batch } \\
=1\end{array}$ & $\begin{array}{l}\text { Trunc } \\
\text { Batch } \\
=5\end{array}$ & $\begin{array}{l}\text { Trunc } \\
\text { Batch } \\
=10\end{array}$ \\
\hline $\begin{array}{l}\text { Mean } \\
\text { Batch } \\
=1\end{array}$ & $\mathbf{- 0 . 1 8 5 4}$ & -0.1782 & -0.0958 \\
\hline $\begin{array}{l}\text { Mean } \\
\text { Batch } \\
=5\end{array}$ & -0.0969 & $\mathbf{- 0 . 1 5 3 1}$ & -0.0767 \\
\hline $\begin{array}{l}\text { Mean } \\
\text { Batch } \\
=10\end{array}$ & -0.0268 & -0.0685 & $\mathbf{- 0 . 0 3 4 4}$ \\
\hline
\end{tabular}

$n=300$

\begin{tabular}{|l|l|l|l|}
\hline & $\begin{array}{l}\text { Trunc } \\
\text { Batch } \\
=1\end{array}$ & $\begin{array}{l}\text { Trunc } \\
\text { Batch } \\
=5\end{array}$ & $\begin{array}{l}\text { Trunc } \\
\text { Batch } \\
=10\end{array}$ \\
\hline $\begin{array}{l}\text { Mean } \\
\text { Batch } \\
=1\end{array}$ & $\mathbf{0 . 0 2 4 5}$ & 0.0221 & -0.0122 \\
\hline $\begin{array}{l}\text { Mean } \\
\text { Batch } \\
=5\end{array}$ & 0.0237 & $\mathbf{0 . 0 0 9 7}$ & 0.0024 \\
\hline $\begin{array}{l}\text { Mean } \\
\text { Batch } \\
=10\end{array}$ & 0.0241 & 0.0165 & $\mathbf{- 0 . 0 2 3 3}$ \\
\hline
\end{tabular}

$n=8000$

\begin{tabular}{|l|l|l|l|}
\hline & $\begin{array}{l}\text { Trunc } \\
\text { Batch } \\
=1\end{array}$ & $\begin{array}{l}\text { Trunc } \\
\text { Batch } \\
=5\end{array}$ & $\begin{array}{l}\text { Trunc } \\
\text { Batch } \\
=10\end{array}$ \\
\hline $\begin{array}{l}\text { Mean } \\
\text { Batch } \\
=1\end{array}$ & $\mathbf{- 0 . 0 5 6 6}$ & -0.0549 & -0.0201 \\
\hline $\begin{array}{l}\text { Mean } \\
\text { Batch } \\
=5\end{array}$ & -0.0595 & $\mathbf{- 0 . 0 7 5 5}$ & -0.0307 \\
\hline $\begin{array}{l}\text { Mean } \\
\text { Batch } \\
=10\end{array}$ & -0.0037 & -0.0193 & $\mathbf{- 0 . 0 4 2 0}$ \\
\hline
\end{tabular}

$n=1000$

\begin{tabular}{|l|l|l|l|}
\hline & $\begin{array}{l}\text { Trunc } \\
\text { Batch } \\
=1\end{array}$ & $\begin{array}{l}\text { Trunc } \\
\text { Batch } \\
=5\end{array}$ & $\begin{array}{l}\text { Trunc } \\
\text { Batch } \\
=10\end{array}$ \\
\hline $\begin{array}{l}\text { Mean } \\
\text { Batch } \\
=1\end{array}$ & $\mathbf{- 0 . 3 7 3 8}$ & -0.1403 & 0.0159 \\
\hline $\begin{array}{l}\text { Mean } \\
\text { Batch } \\
=5\end{array}$ & -0.0987 & $\mathbf{- 0 . 1 4 6 3}$ & 0.0115 \\
\hline $\begin{array}{l}\text { Mean } \\
\text { Batch } \\
=10\end{array}$ & -0.0165 & 0.0275 & $\mathbf{0 . 0 9 4 6}$ \\
\hline
\end{tabular}

$n=150$

\begin{tabular}{|l|l|l|l|}
\hline & $\begin{array}{l}\text { Trunc } \\
\text { Batch } \\
=1\end{array}$ & $\begin{array}{l}\text { Trunc } \\
\text { Batch } \\
=5\end{array}$ & $\begin{array}{l}\text { Trunc } \\
\text { Batch } \\
=10\end{array}$ \\
\hline $\begin{array}{l}\text { Mean } \\
\text { Batch } \\
=1\end{array}$ & $\mathbf{0 . 0 0 2 3}$ & 0.0051 & 0.0102 \\
\hline $\begin{array}{l}\text { Mean } \\
\text { Batch } \\
=5\end{array}$ & 0.0015 & $\mathbf{- 0 . 0 0 8 8}$ & 0.0004 \\
\hline $\begin{array}{l}\text { Mean } \\
\text { Batch } \\
=10\end{array}$ & 0.0010 & -0.0058 & $\mathbf{- 0 . 0 1 1 4}$ \\
\hline
\end{tabular}

$n=4000$

\begin{tabular}{|l|l|l|l|}
\hline & $\begin{array}{l}\text { Trunc } \\
\text { Batch } \\
=1\end{array}$ & $\begin{array}{l}\text { Trunc } \\
\text { Batch } \\
=5\end{array}$ & $\begin{array}{l}\text { Trunc } \\
\text { Batch } \\
=10\end{array}$ \\
\hline $\begin{array}{l}\text { Mean } \\
\text { Batch } \\
=1\end{array}$ & $\mathbf{- 0 . 1 8 5 5}$ & -0.1398 & -0.0686 \\
\hline $\begin{array}{l}\text { Mean } \\
\text { Batch } \\
=5\end{array}$ & -0.1211 & $\mathbf{- 0 . 1 4 1 8}$ & -0.0677 \\
\hline $\begin{array}{l}\text { Mean } \\
\text { Batch } \\
=10\end{array}$ & -0.0647 & -0.0736 & $\mathbf{- 0 . 0 4 3 9}$ \\
\hline
\end{tabular}

$$
n=500
$$

\begin{tabular}{|l|l|l|l|}
\hline & $\begin{array}{l}\text { Trunc } \\
\text { Batch } \\
=1\end{array}$ & $\begin{array}{l}\text { Trunc } \\
\text { Batch } \\
=5\end{array}$ & $\begin{array}{l}\text { Trunc } \\
\text { Batch } \\
=10\end{array}$ \\
\hline $\begin{array}{l}\text { Mean } \\
\text { Batch } \\
=1\end{array}$ & $\mathbf{- 0 . 2 8 4 7}$ & -0.0222 & 0.0204 \\
\hline $\begin{array}{l}\text { Mean } \\
\text { Batch } \\
=5\end{array}$ & 0.0231 & $\mathbf{- 0 . 0 1 3 2}$ & 0.0129 \\
\hline $\begin{array}{l}\text { Mean } \\
\text { Batch } \\
=10\end{array}$ & 0.0452 & 0.0239 & $\mathbf{0 . 0 4 7 4}$ \\
\hline
\end{tabular}

$n=100$ 
- Batch Size of 1 - Batch Size of 5 - Batch Size of 10

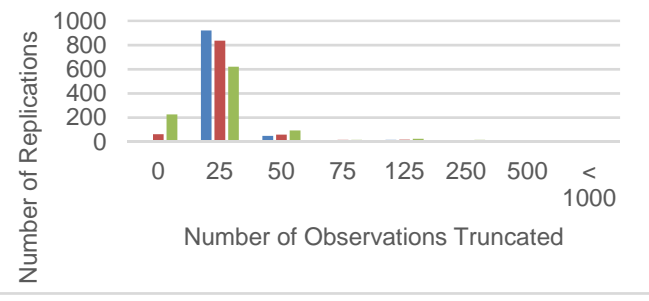

$n=16000$

Batch Size of 1 Batch Size of 5 Batch Size of 10

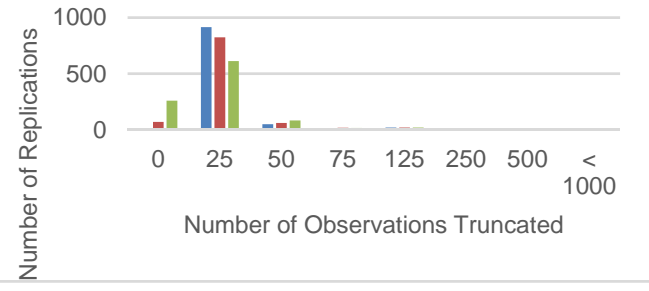

$$
n=2000
$$

Batch Size of 1 Batch Size of 5 Batch Size of 10

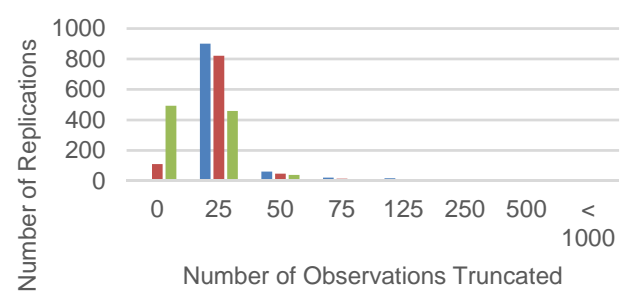

$n=300$
- Batch Size of 1 - Batch Size of 5 - Batch Size of 10

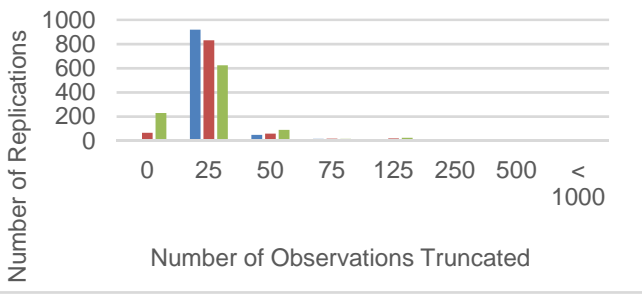

$n=8000$

Batch Size of 1 Batch Size of 5 Batch Size of 10

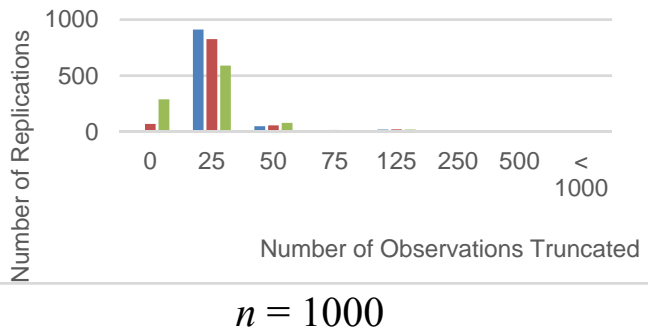

घatch Size of 1 Batch Size of 5 Batch Size of 10

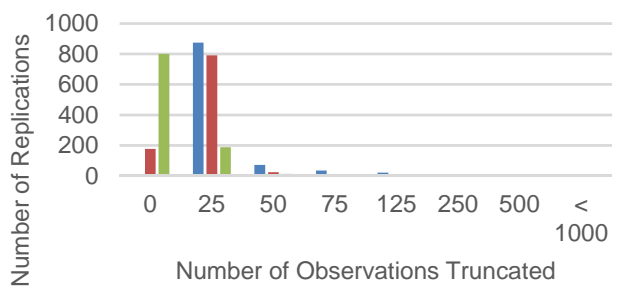

$n=150$
- Batch Size of 1 - Batch Size of 5 - Batch Size of 10

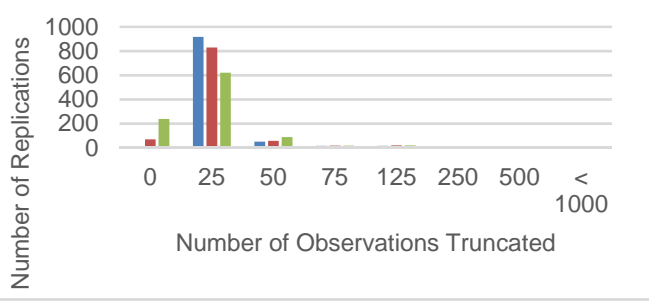

$n=4000$

-Batch Size of 1 Batch Size of $5 \square$ Batch Size of 10

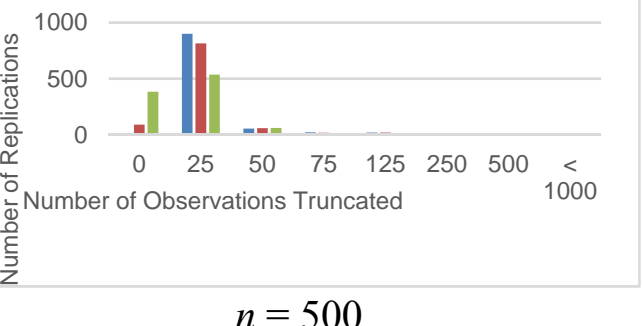

Batch Size of 1 Batch Size of 5 Batch Size of 10

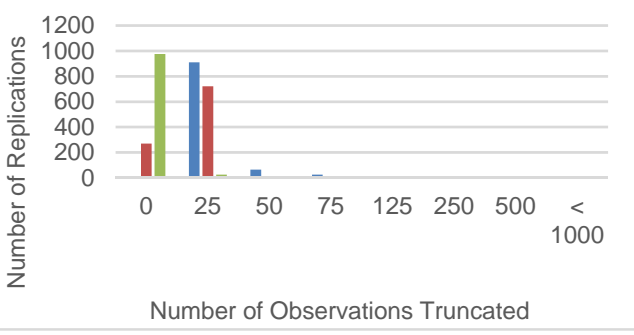

$n=100$

Figure 7.65 Frequency distribution of the number of observations truncated as a function of batch sizes $b=1,5$, and 10 for run length of $n=100,150,300,500,1000,2000,4000,8000$, and 16,000 for Model 3 with $\phi$ of 0.80 


\subsubsection{Results for Model 3 with $\phi=0.70$}

$\mathrm{n}=16000$ and param $=0.7$

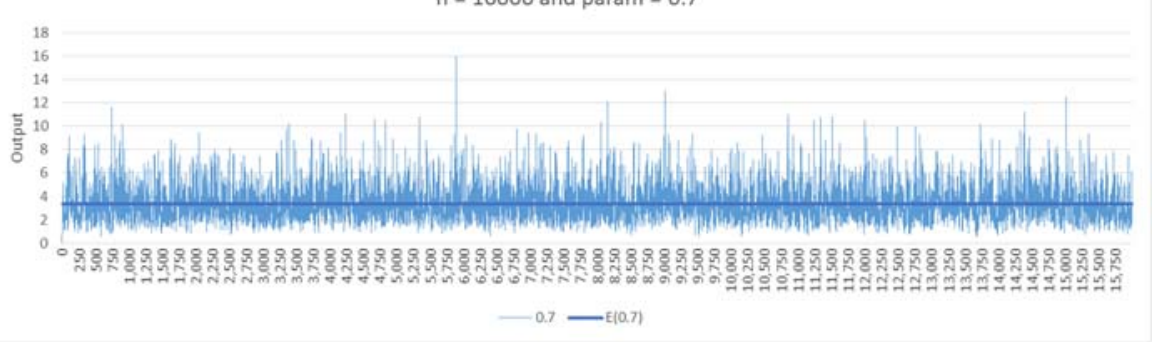

Figure 7.66 Example of EAR(1) with $\phi$ of $0.7(n=16000$, Blue line: $E(X))$
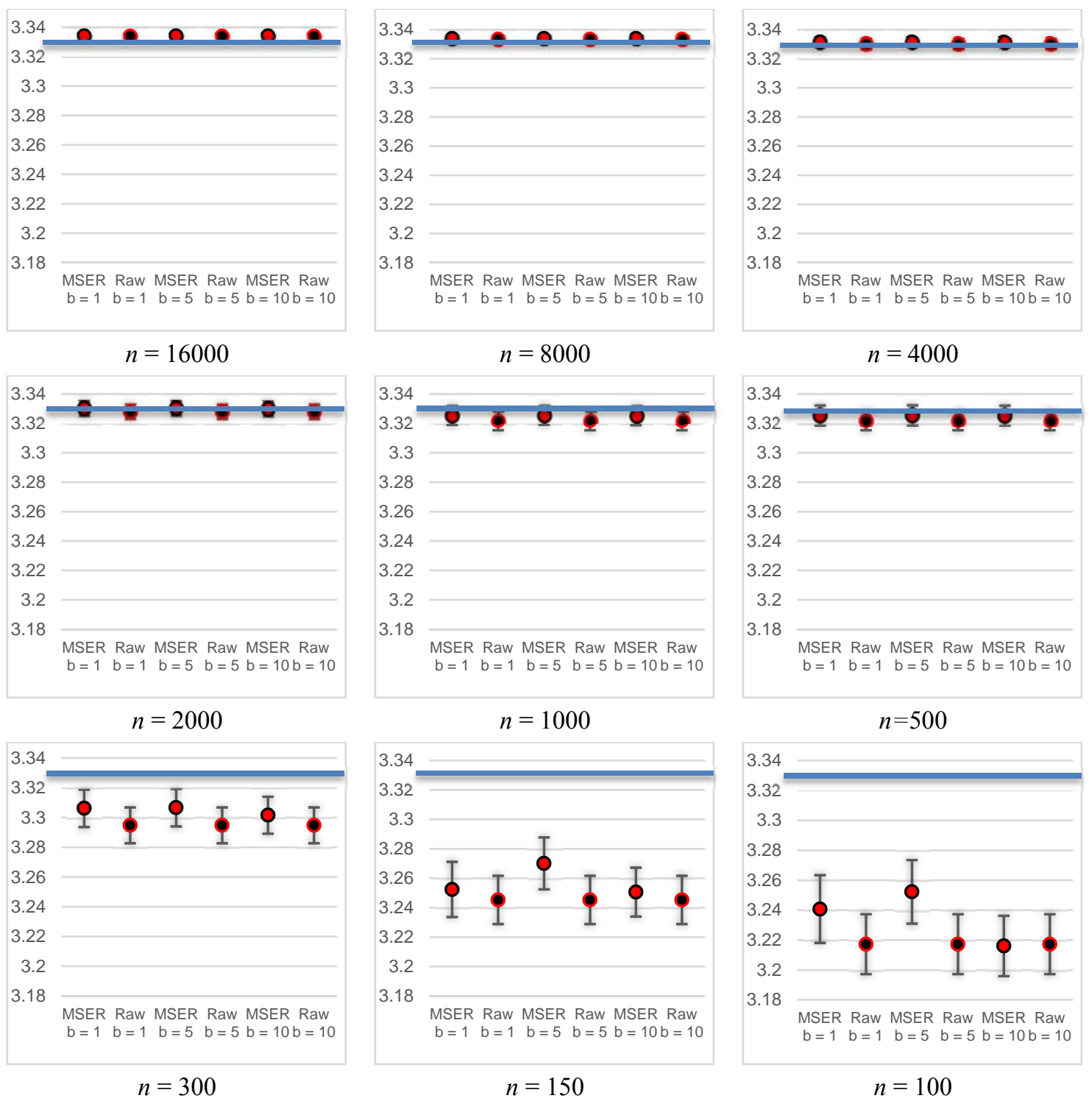

Figure $7.6795 \%$ confidence intervals of the mean for the truncated mean output as a function of batch size $(b=1,5$, and 10) and run length $(n=100,150,300,500,1000,2000$, 4000,8000 , and 16,000) for Model 3 with $\phi=0.70$ (Theoretical mean of 3.33, Blue line) 

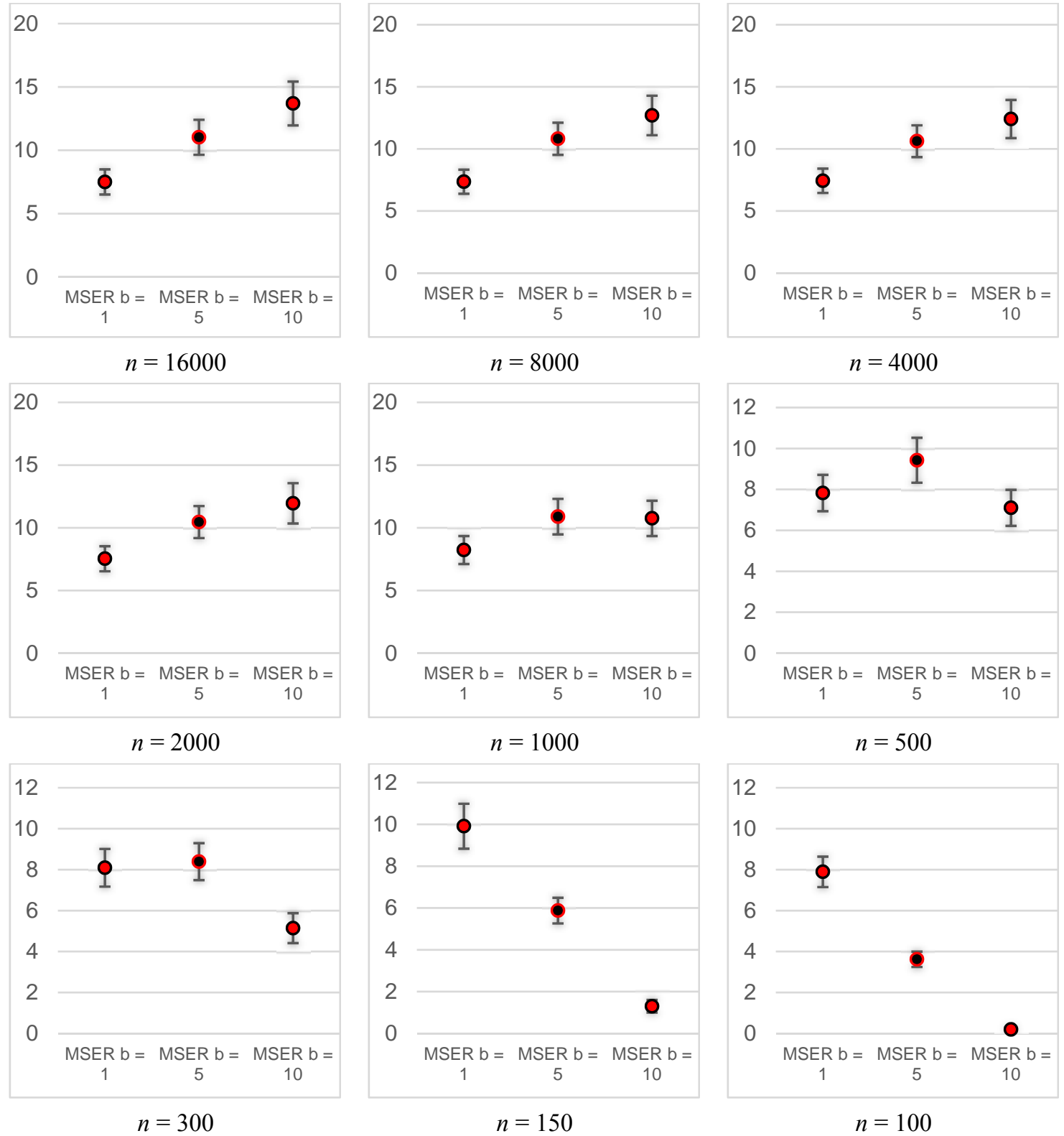

Figure $7.6895 \%$ confidence intervals for the mean number of observations truncated as a function of batch size $(b=1,5$, and 10) and run length $(n=100,150,300,500,1000,2000$, 4000,8000 , and 16,000) for Model 3 with $\phi=0.70$ 

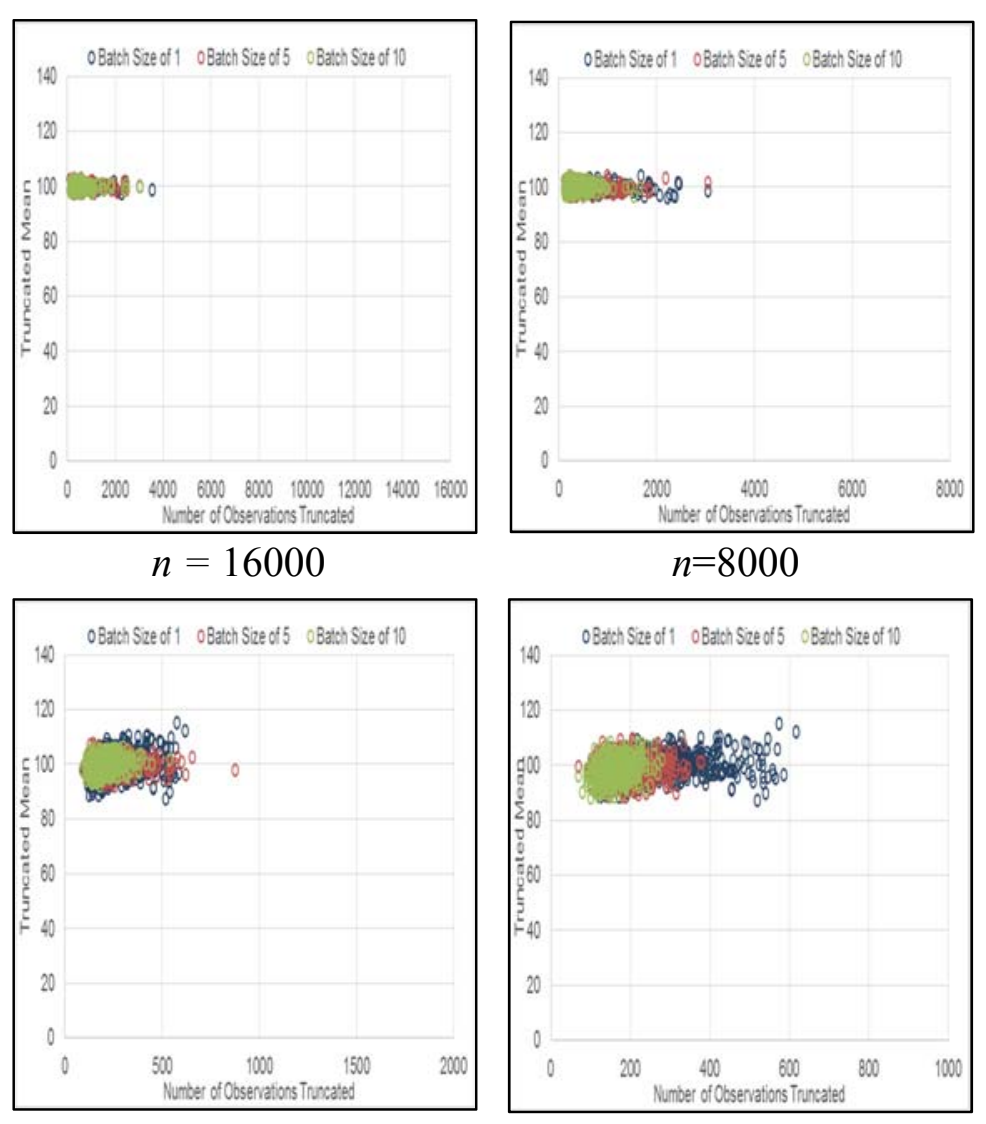

$n=2000$

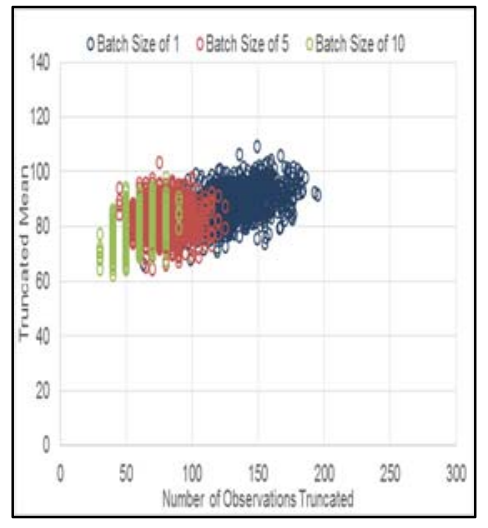

$n=300$ $n=8000$

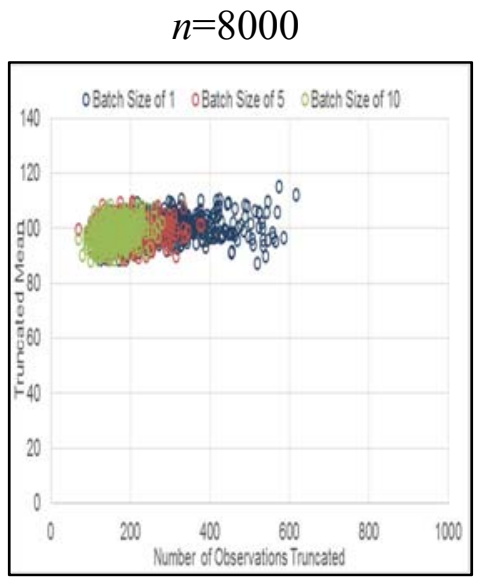

$$
n=1000
$$

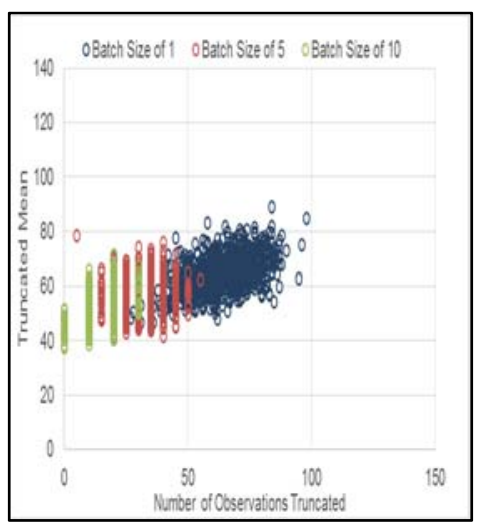

$n=150$

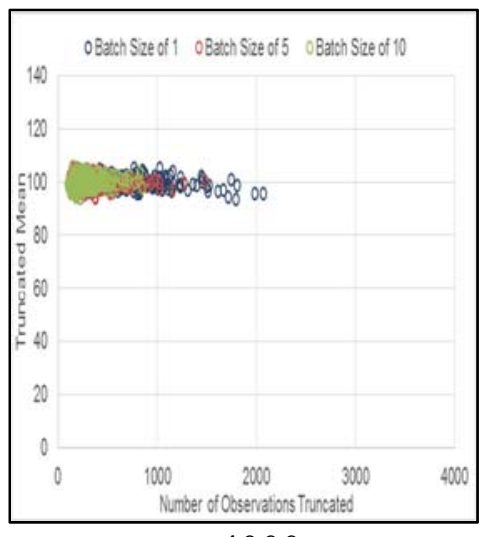

$n=4000$

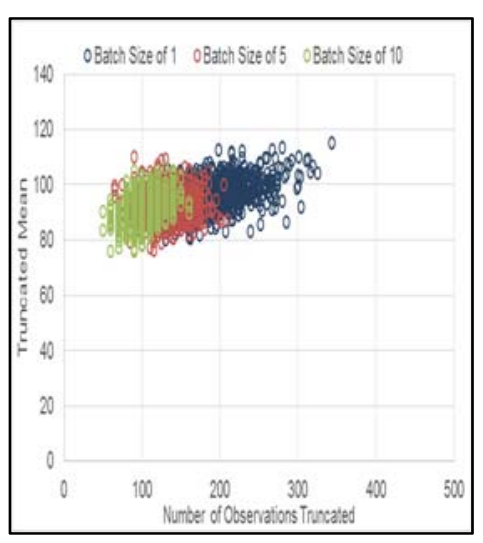

$n=500$

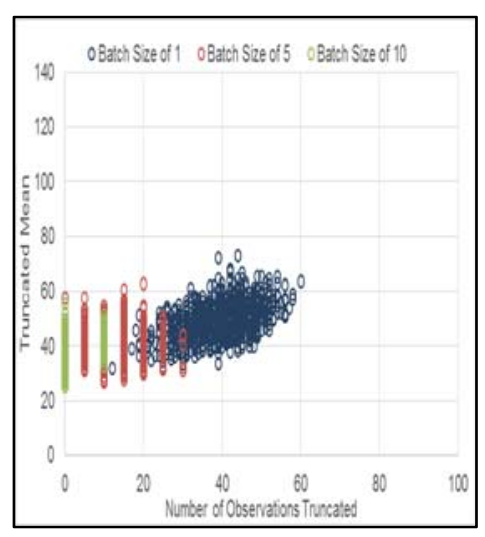

$n=100$

Figure 7.69 Scatterplots of the truncated mean vs. the number of observations truncated for batch sizes $b=1,5$, and 10 for run length of $n=100,150,300,500,1000,2000,4000,8000$, and 16,000 Model 3 with $\phi=0.70$ 
Table 7.28 Correlation between the truncated mean and the number of observations truncated for batch sizes $b=1,5$, and 10 for run length of $n=100,150,300,500,1000,2000$, 4000, 8000, and 16,000 for Model 3 with $\phi=0.70$

\begin{tabular}{|l|l|l|l|}
\hline & $\begin{array}{l}\text { Trunc } \\
\text { Batch } \\
=1\end{array}$ & $\begin{array}{l}\text { Trunc } \\
\text { Batch } \\
=5\end{array}$ & $\begin{array}{l}\text { Trunc } \\
\text { Batch } \\
=10\end{array}$ \\
\hline $\begin{array}{l}\text { Mean } \\
\text { Batch } \\
=1\end{array}$ & $\mathbf{0 . 0 5 3 3}$ & 0.0414 & 0.0544 \\
\hline $\begin{array}{l}\text { Mean } \\
\text { Batch } \\
=5\end{array}$ & 0.0534 & $\mathbf{0 . 0 3 2 0}$ & 0.0496 \\
\hline $\begin{array}{l}\text { Mean } \\
\text { Batch } \\
=10\end{array}$ & 0.0549 & 0.0362 & $\mathbf{0 . 0 4 4 5}$ \\
\hline
\end{tabular}

$n=16000$

\begin{tabular}{|l|l|l|l|}
\hline & $\begin{array}{l}\text { Trunc } \\
\text { Batch } \\
=1\end{array}$ & $\begin{array}{l}\text { Trunc } \\
\text { Batch } \\
=5\end{array}$ & $\begin{array}{l}\text { Trunc } \\
\text { Batch } \\
=10\end{array}$ \\
\hline $\begin{array}{l}\text { Mean } \\
\text { Batch } \\
=1\end{array}$ & $\mathbf{- 0 . 0 1 5 9 0}$ & 0.00987 & -0.01131 \\
\hline $\begin{array}{l}\text { Mean } \\
\text { Batch } \\
=5\end{array}$ & -0.01121 & $\mathbf{- 0 . 0 1 4 2 0}$ & -0.02741 \\
\hline $\begin{array}{l}\text { Mean } \\
\text { Batch } \\
=10\end{array}$ & -0.01241 & -0.00999 & $\mathbf{- 0 . 0 4 3 1 3}$ \\
\hline
\end{tabular}

$$
n=2000
$$

\begin{tabular}{|l|l|l|l|}
\hline & $\begin{array}{l}\text { Trunc } \\
\text { Batch } \\
=1\end{array}$ & $\begin{array}{l}\text { Trunc } \\
\text { Batch } \\
=5\end{array}$ & $\begin{array}{l}\text { Trunc } \\
\text { Batch } \\
=10\end{array}$ \\
\hline $\begin{array}{l}\text { Mean } \\
\text { Batch } \\
=1\end{array}$ & $\mathbf{- 0 . 1 8 8 9}$ & -0.1540 & -0.1375 \\
\hline $\begin{array}{l}\text { Mean } \\
\text { Batch } \\
=5\end{array}$ & -0.1520 & $\mathbf{- 0 . 1 6 4 2}$ & -0.1320 \\
\hline $\begin{array}{l}\text { Mean } \\
\text { Batch } \\
=10\end{array}$ & -0.0862 & -0.0812 & $\mathbf{- 0 . 1 1 7 5}$ \\
\hline
\end{tabular}

$n=300$

\begin{tabular}{|l|l|l|l|}
\hline & $\begin{array}{l}\text { Trunc } \\
\text { Batch } \\
=1\end{array}$ & $\begin{array}{l}\text { Trunc } \\
\text { Batch } \\
=5\end{array}$ & $\begin{array}{l}\text { Trunc } \\
\text { Batch } \\
=10\end{array}$ \\
\hline $\begin{array}{l}\text { Mean } \\
\text { Batch } \\
=1\end{array}$ & $\mathbf{0 . 0 3 2 2}$ & 0.0211 & 0.0171 \\
\hline $\begin{array}{l}\text { Mean } \\
\text { Batch } \\
=5\end{array}$ & 0.0324 & $\mathbf{0 . 0 1 0 8}$ & 0.0101 \\
\hline $\begin{array}{l}\text { Mean } \\
\text { Batch } \\
=10\end{array}$ & 0.0353 & 0.0159 & $\mathbf{0 . 0 0 4 6}$ \\
\hline
\end{tabular}

$n=8000$

\begin{tabular}{|l|l|l|l|}
\hline & $\begin{array}{l}\text { Trunc } \\
\text { Batch } \\
=1\end{array}$ & $\begin{array}{l}\text { Trunc } \\
\text { Batch } \\
=5\end{array}$ & $\begin{array}{l}\text { Trunc } \\
\text { Batch } \\
=10\end{array}$ \\
\hline $\begin{array}{l}\text { Mean } \\
\text { Batch } \\
=1\end{array}$ & $\mathbf{- 0 . 0 4 8 9}$ & -0.0357 & -0.0050 \\
\hline $\begin{array}{l}\text { Mean } \\
\text { Batch } \\
=5\end{array}$ & -0.0433 & $\mathbf{- 0 . 0 7 0 7}$ & -0.0243 \\
\hline $\begin{array}{l}\text { Mean } \\
\text { Batch } \\
=10\end{array}$ & 0.0036 & -0.0122 & $\mathbf{- 0 . 0 2 3 5}$ \\
\hline
\end{tabular}

$$
n=1000
$$

\begin{tabular}{|l|l|l|l|}
\hline & $\begin{array}{l}\text { Trunc } \\
\text { Batch } \\
=1\end{array}$ & $\begin{array}{l}\text { Trunc } \\
\text { Batch } \\
=5\end{array}$ & $\begin{array}{l}\text { Trunc } \\
\text { Batch } \\
=10\end{array}$ \\
\hline $\begin{array}{l}\text { Mean } \\
\text { Batch } \\
=1\end{array}$ & $\mathbf{- 0 . 4 0 4 2}$ & -0.1781 & -0.0367 \\
\hline $\begin{array}{l}\text { Mean } \\
\text { Batch } \\
=5\end{array}$ & -0.1494 & $\mathbf{- 0 . 1 8 5 5}$ & -0.0261 \\
\hline $\begin{array}{l}\text { Mean } \\
\text { Batch } \\
=10\end{array}$ & -0.0409 & 0.0120 & $\mathbf{- 0 . 0 0 1 5}$ \\
\hline
\end{tabular}

$n=150$

\begin{tabular}{|l|l|l|l|}
\hline & $\begin{array}{l}\text { Trunc } \\
\text { Batch } \\
=1\end{array}$ & $\begin{array}{l}\text { Trunc } \\
\text { Batch } \\
=5\end{array}$ & $\begin{array}{l}\text { Trunc } \\
\text { Batch } \\
=10\end{array}$ \\
\hline $\begin{array}{l}\text { Mean } \\
\text { Batch } \\
=1\end{array}$ & $\mathbf{- 0 . 0 2 4 8}$ & 0.0189 & 0.0068 \\
\hline $\begin{array}{l}\text { Mean } \\
\text { Batch } \\
=5\end{array}$ & -0.0238 & $\mathbf{0 . 0 0 2 5}$ & -0.0054 \\
\hline $\begin{array}{l}\text { Mean } \\
\text { Batch } \\
=10\end{array}$ & -0.0196 & 0.0093 & $\mathbf{- 0 . 0 0 8 6}$ \\
\hline
\end{tabular}

$n=4000$

\begin{tabular}{|l|l|l|l|}
\hline & $\begin{array}{l}\text { Trunc } \\
\text { Batch } \\
=1\end{array}$ & $\begin{array}{l}\text { Trunc } \\
\text { Batch } \\
=5\end{array}$ & $\begin{array}{l}\text { Trunc } \\
\text { Batch } \\
=10\end{array}$ \\
\hline $\begin{array}{l}\text { Mean } \\
\text { Batch } \\
=1\end{array}$ & $\mathbf{- 0 . 1 1 2 3}$ & -0.0845 & -0.0460 \\
\hline $\begin{array}{l}\text { Mean } \\
\text { Batch } \\
=5\end{array}$ & -0.0980 & $\mathbf{- 0 . 1 3 2 0}$ & -0.0551 \\
\hline $\begin{array}{l}\text { Mean } \\
\text { Batch } \\
=10\end{array}$ & -0.0548 & -0.0555 & $\mathbf{- 0 . 0 3 9 6}$ \\
\hline
\end{tabular}

$n=500$

\begin{tabular}{|l|c|l|l|}
\hline & $\begin{array}{l}\text { Trunc } \\
\text { Batch } \\
=1\end{array}$ & $\begin{array}{l}\text { Trunc } \\
\text { Batch } \\
=5\end{array}$ & $\begin{array}{l}\text { Trunc } \\
\text { Batch } \\
=10\end{array}$ \\
\hline $\begin{array}{l}\text { Mean } \\
\text { Batch } \\
=1\end{array}$ & $\mathbf{- 0 . 3 4 7 9}$ & -0.1125 & -0.0772 \\
\hline $\begin{array}{l}\text { Mean } \\
\text { Batch } \\
=5\end{array}$ & -0.0362 & $\mathbf{- 0 . 0 8 1 8}$ & -0.0866 \\
\hline $\begin{array}{l}\text { Mean } \\
\text { Batch } \\
=10\end{array}$ & 0.0228 & -0.0025 & $\mathbf{- 0 . 0 6 6 7}$ \\
\hline
\end{tabular}

$n=100$ 


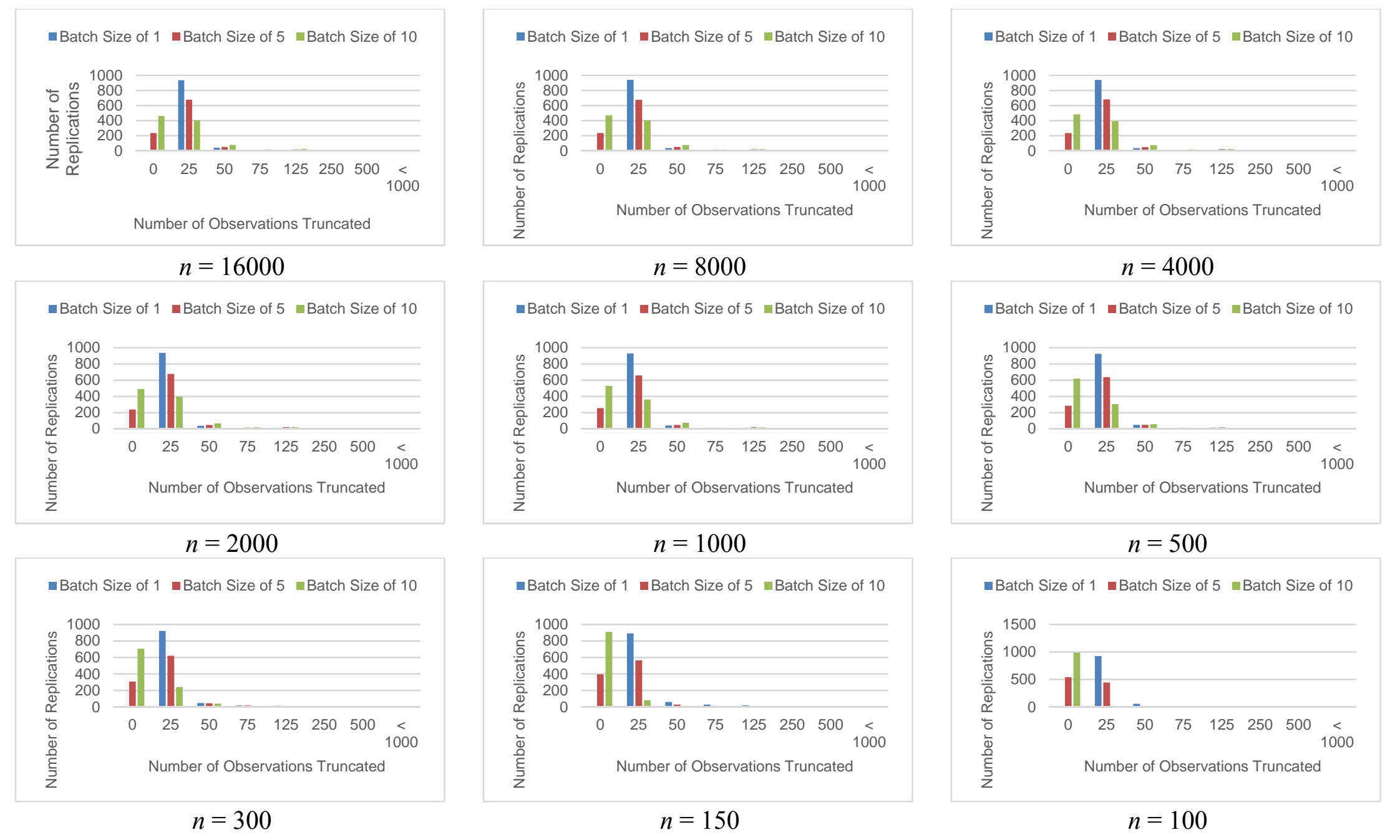

Figure 7.70 Frequency distribution of the number of observations truncated as a function of batch sizes $b=1,5$, and 10 for run length of $n=100,150,300,500,1000,2000,4000,8000$, and 16,000 for Model 3 with ${ }^{\phi}$ of 0.70 


\subsubsection{Results Using OBM for Model 3 with $\phi=0.70$}

We applied OBM to Model 3 with run length $n=1000$ for batch sizes $b=10,50,100$, and 200 observations. Figure 7.71 shows output for four representative replications, one at each batch size. Table 7.29 and Figure 7.72 (a) compares the $95 \%$ confidence intervals on the truncated mean for each of the OBM estimates with the truncated mean for nonoverlapping approach with the single batch size $b=10$. None of the interval estimates cover the expected value of 3.33 except OBM $b=100$ and NOBM. However, all the mean estimates are within $0.3 \%$ of the theoretical mean and the differences among these estimates are not statistically significant.
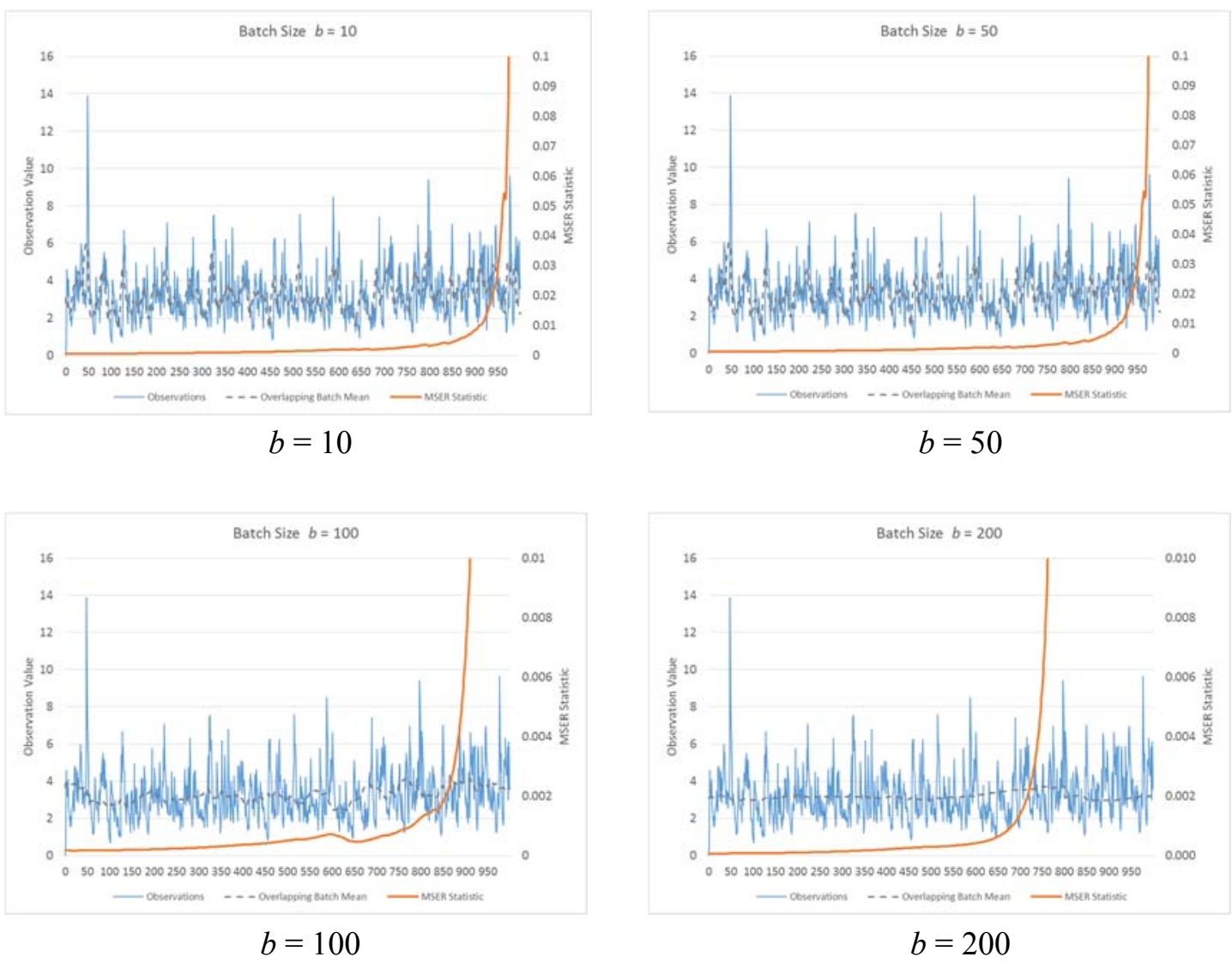

Figure 7.71 Representative Output, Overlapping Batch Mean, and MSER Statistic of Model $3(b=10,50,100$, and 200) 
Table 7.29 95\% confidence intervals for Model 3 on the truncated mean and the standard deviation for overlapping and non-overlapping batches

\begin{tabular}{|c|c|c|c|c|c|}
\hline & \multicolumn{4}{|c|}{ OBM } & NOBM \\
\cline { 2 - 6 } & OBM $b=10$ & OBM $b=50$ & OBM $b=100$ & OBM $b=200$ & NOBM $b=10$ \\
\hline Sample Mean & 3.322456 & 3.320894 & 3.323329 & 3.322423 & 3.325367 \\
\hline Upper limit & 3.329200 & 3.327986 & 3.330414 & 3.329345 & 3.332040 \\
\hline Lower limit & 3.315712 & 3.313801 & 3.316244 & 3.315501 & 3.318693 \\
\hline Sample Std D & 0.108683 & 0.114299 & 0.114175 & 0.111547 & 0.107546 \\
\hline Upper limit & 0.113668 & 0.119542 & 0.119412 & 0.116663 & 0.112479 \\
\hline Lower limit & 0.104120 & 0.109500 & 0.109381 & 0.106863 & 0.103031 \\
\hline
\end{tabular}

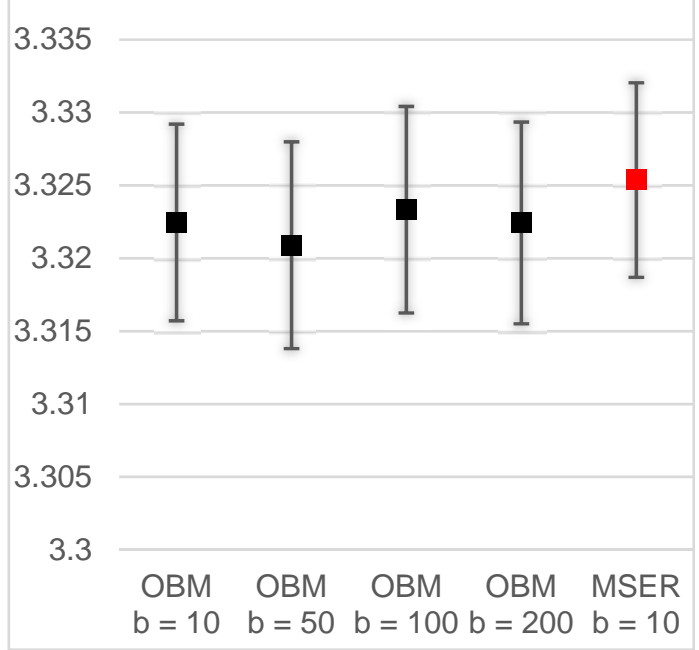

(a)

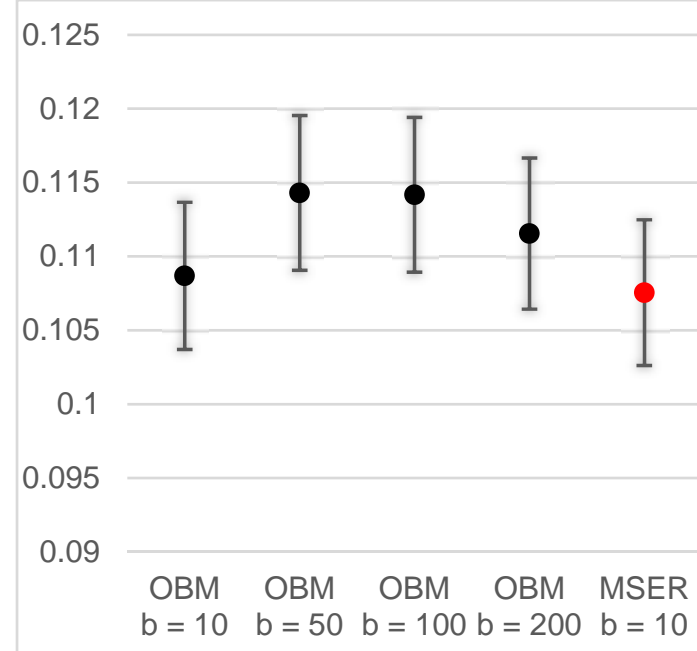

(b)

Figure 7.72 95\% confidence intervals for Model 3 on (a) the truncated mean for overlapping and non-overlapping batches and (b) the standard deviation for overlapping and nonoverlapping batches

Figure 7.73, 7.74, and Table 7.29 suggest an interesting finding of how OBM does act compared to NOBM. As we see in Model 2, this approach keeps looking for the minimal MSER statistic over $d^{*}<n / 2$ and this tendency causes violation of the half run rule. Thus, imposing this rule for OBM should be recommended. In addition, OBM $b=10$ and NOBM $b=10$ perform similar patterns as the pre-processed output from OBM follows NOBM's output trend. 


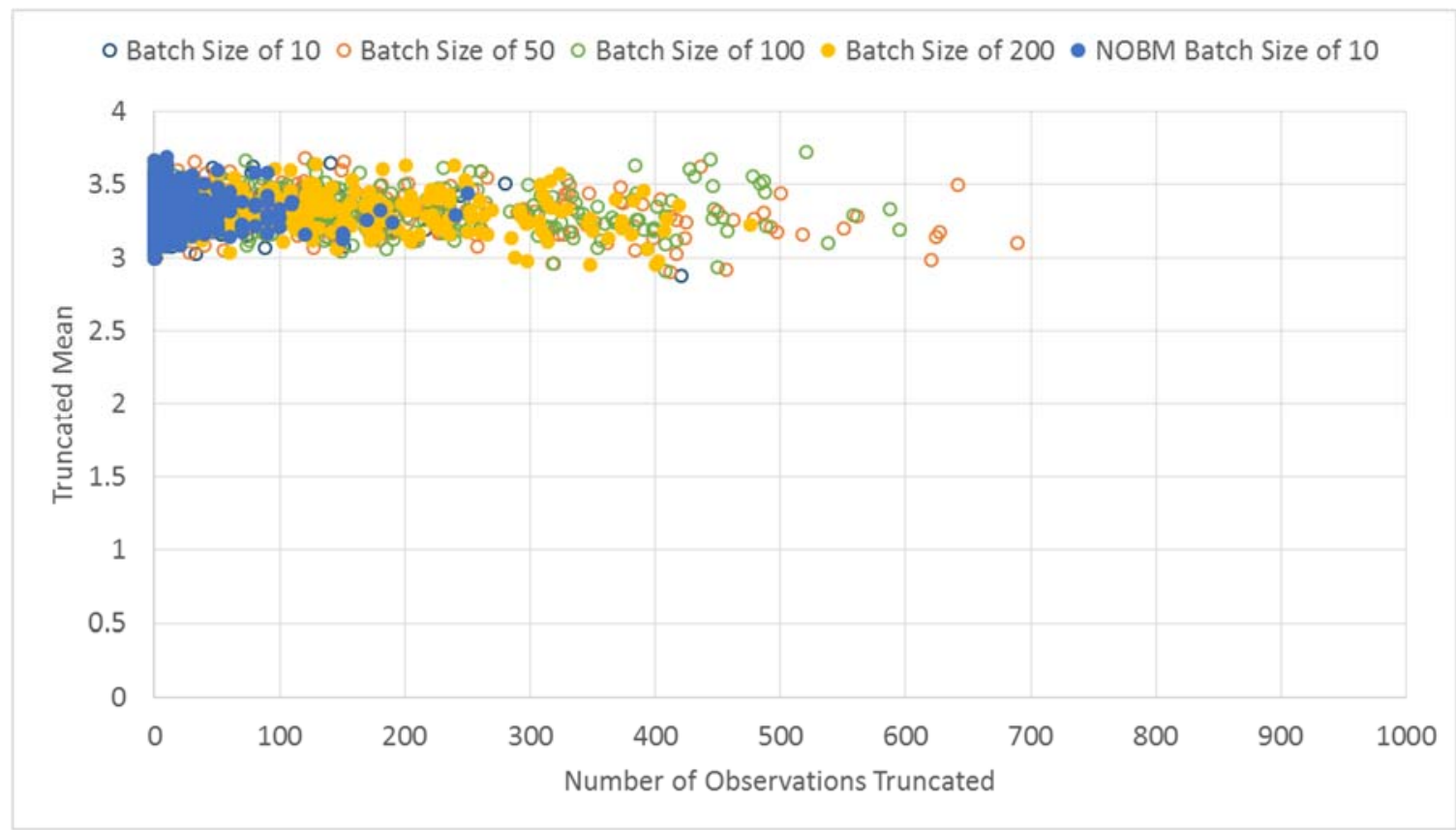

Figure 7.73 Scatterplots of the truncated mean vs. the number of observations truncated for OBM sizes $b=10,50,100$ and 200 for run length of $n=1000$ with $\phi$ of 0.70 for Model 3 (including NOBM batch size of 10)

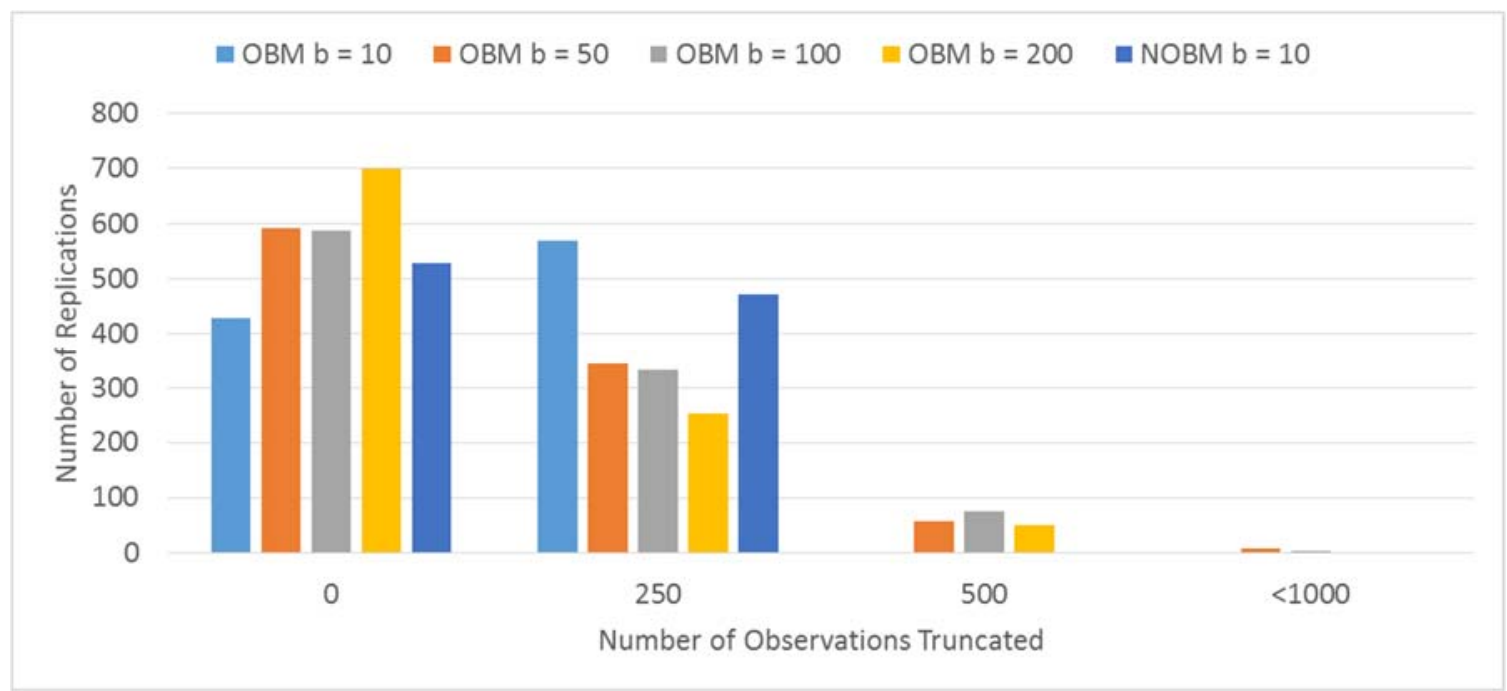

Figure 7.74 Frequency distribution of the number of observations truncated as a function of OBM sizes $b=10,50,100$, and 200 for run length of $n=1000$ with $\phi$ of 0.70 for Model 3 (including NOBM batch size of 10) 
Table 7.30 Correlation between the truncated mean and the number of observations truncated for OBM sizes $b=10,50,100$, and 200 for run length of $n=16,000$ with $\phi$ of 0.70 for Model 3

\begin{tabular}{|l|c|c|c|c|}
\hline & Trunc Batch =10 & Trunc Batch =50 & Trunc Batch =100 & Trun Batch = 200 \\
\hline Mean Batch $=10$ & -0.105123784 & 0.024183645 & -0.007825651 & -0.041654617 \\
\hline Mean Batch $=50$ & 0.005546592 & -0.144419915 & -0.01367133 & -0.069917161 \\
\hline Mean Batch $=100$ & -0.062115342 & 0.031223516 & -0.036393455 & -0.036956424 \\
\hline Mean Batch $=200$ & -0.018985329 & -0.022951969 & -0.003507772 & -0.103548868 \\
\hline
\end{tabular}




\section{Chapter 8. Conclusion and future research}

One measure of the effectiveness of applied research on DES is the degree to which research findings are incorporated into standard practice. A major purpose of our research was to enhance the effectiveness of MSER in just this way. Two different approaches to improving MSER accessibly were implemented and thoroughly tested.

The first was to integrate MSER logic in commercial simulation software in the form of a static library, a DLL, and a submodel (subroutine); the second was to provide standalone codes a variety of programming language to use as MSER post-analysis. After extensive reviews, we chose the commercial simulation software ExtendSim, ProModel/MedModel, and Arena to realize this first approach; we chose R, SAS, Matlab, VBA, and $\mathrm{C} / \mathrm{C}++$ to implement the second.

To make these codes public and facilitate distribution, we created the MSER Laboratory hosted at the University of Virginia. In addition to the sample codes, this web site currently includes information on the mathematical and historical development of MSER, as well as references into the literature. Our intention is to maintain and support the continued development and expansion of the Lab.

In the future, we will explore modifications to the laboratory site which will make it interactive, such that a warm-up period can be determined automatically simply by importing a data set to be analyzed. We also intend to include codes in additional programming languages, such as the scripting languages Python and Perl, as well as 
incorporate useful suggestions and related research findings from the simulation community. We are very anxious to work with developers of commercial simulation software houses to incorporate MSER in future releases of their products, as we have done previously with Dave Krahl at Imagine That (to whom we reiterate our sincere thanks and appreciation). We also intend to pursue research to advance the MSER logic module, particularly with regard to integrating MSER with the notion of automatic stopping rules.

The second major purpose of our research was to address a number of open questions regarding MSER application and provide guidance in the selection of MSER parameters. These include:

- the choice of simulation run length $n$,

- the choice of batch size $b$,

- the maximum acceptable optimal truncation point $d_{\max }$ on the range of a given run length $\left[0 \leq d_{\max } \leq n\right]$, and

- the incorporation of the overlapping batch means.

To this end we first develop a hypothetical case which demonstrated that MSER can potentially change its determinations regarding the location and the even the existence of a suitable truncation point depending on the run length chosen. We concluded that performance of MSER does in fact depend fundamentally on choosing a sufficient run length. Without knowledge beyond the output sequences alone, this choice remains an open problem and the subject for future research.

To develop insight regarding the remaining questions, we tested MSER using nonoverlapping batch means (NOBM) with batch sizes of $b=1,5$, and 10 and selected run lengths for three simulation models: (1) a uniform white noise process with superimposed 
linearly decreasing bias, (2) the delay time in $\mathrm{M} / \mathrm{M} / 1$ with four different traffic intensities, and (3) EAR(1) with four different parameters. We also tested MSER using overlapping batch means (OBM) with selected batch sizes run lengths.

We selected Model 1 as the baseline for testing MSER performance because of its transparency - the range of optimal truncation points is clear, both visually and analytically. We showed that for long runs:

(1) all batch sizes remove all of the transient observations,

(2) estimation errors are an artifact of sampling after the biasing effect of the initial transient has been removed,

(3) modest batching has no significant effect on the quality of estimates, and

(4) the mean estimate is uncorrelated with the number of observations truncated for all the batch sizes and the success of a truncation procedure in terms of the accuracy of the estimate cannot be imputed from the truncation point alone.

For shorter runs, we showed conclusion (4) also holds. In addition:

(5) even with very little steady-state data, the MSER-indicated truncation points are themselves very reasonable and indeed optimal in terms of the mean estimates for most cases,

(6) increasing batch sizes increases both the variance and spread of the truncated observations, without systematically affecting the accuracy of the estimated mean,

(7) to the degree that batching reduces the effective sample size, it is not recommended for small samples and provides no discernable benefit for large samples, 
(8) the choice of $d_{\max }$ is a binding concern only if (n-d)/n is close to 1 - the choice of $n$ is likely dominated by the need for estimates with acceptable accuracy and precision,

(9) the $d^{*} \leq d_{\max }=n / 2$ threshold provides significant protection against estimation errors resulting from run lengths that are too short without over-truncation of replications with adequate run lengths,

(10) for run lengths that are approximately the same as ideal truncation point, however, the protection may be inadequate and a modestly lower threshold would be preferred, and

(11) OBM outperforms the non-overlapping approach in all cases and OBM offers significantly greater precision in the estimate.

We selected Model 2 because it allows comparison with the results obtained by Law (2015) and by White and Hwang (2015) for long runs. The results obtained by White and Hwang (2015) demonstrated that truncation points and the error in the truncated mean estimates are essentially independent. Further that, while the mean estimates for this problem are quite good without truncation, applying MSER-5 truncation modestly improves the accuracy of these estimates.

Our objective here was to explore the performance of MSER for a single initial condition, empty and idle $\left\{x_{0}=0\right\}$, with respect to alternative run lengths, batch sizes, and model traffic intensities. We found that:

(1) the results obtained in the present research for $n=64,000$ and $\rho=0.9$ are entirely consistent with those reported earlier. 
(2) while we speculated that the effects of decreasing $\rho$ would be similar to those encountered by increasing the run length $n$, the results of our experiments suggest that this speculation is largely untrue and decreasing traffic intensity cannot altogether compensate for short run length,

(3) the $d^{*} \leq d_{\max }=n / 2$ truncation rule likewise does not altogether compensate for short run lengths, and

(4) for smaller run lengths and traffic intensities, MSER appears to overestimate the amount of truncation warranted and underestimate the steady-state mean.

We speculate that conclusion (4) obtains because truncating early regenerative cycles with large peak waiting times, when these exist, reduces the sample variance. Early in the run, the MSER statistic is relatively less sensitive to the accompanying reduction in sample size than to the reduction of sample variance. This speculation remains open for future research.

In the final analysis, our research demonstrates that

(5) the difficulty in estimating the steady-state mean has little or nothing to do with bias resulting from a poor choice of initial conditions for Model 2. The empty-and-idle condition regenerates frequently, more frequently for lower traffic intensities and smaller batches, and

(6) instead, the fundamental issue here is determining an initial run length that is sufficiently long to capture observations that, taken together, are representative of the steady-state distribution.

In other words, 
(7) for regenerative processes with irregular cyclical outputs and inadequate run lengths, MSER may suggest truncation points when truncation is contraindicated.

Future research might extend these results by testing the effect of alternate initial conditions other than $x_{0}=0$ or 100 , where truncation is actually required to mitigate initialization bias.

We selected Model 3 because determining the "true" truncation point for this model is problematic. Unlike Model 1, the process converges to steady state only in the limit and which observation to choose as the "true" truncation point is inherently subjective. We noted that MSER is data driven and does not suffer from this ambiguity. We also suggested the expected $\gamma$-percent settling time as a measure for the degree of truncation.

We found that:

(1) MSER yields superior estimates for all run lengths, with the difference in accuracy increasing as the run lengths as the decrease,

(2) restricting the optimal truncation point to $d^{*}<n / 2$ does not consistently appear to provide the desired indication that runs are too short to yield accurate estimates on average.

However, we speculate the same is not true when the threshold is applied on a replicationby-replication basis, as recommended. This speculation warrants future research.

(3) Truncated mean estimates are relatively independent of the number of observations truncated - for long runs, the correlation is essentially zero; for medium and short runs, there appears to be a modest positive correlation, with the magnitude of this correlation generally increasing as run length decreases. The shortest runs tend to induce greater truncation on average, and 
(4) NOBM provides better accuracy and precision at $b=10$.

It is unclear whether or not conclusion (4) holds for other batch sizes. Adding clarity on the relative performance of OBM and NOBM is a potentially fruitful subject for future research.

The conclusion consistently obtained in this research - that batching, in general, does not improve estimates and can, in some instances, result in a loss of precision seemingly contradicts a long-standing result in the literature (White, Cobb, and Spratt, 2000) but agrees with Schmeiser's discussion and recommendation

The MSER-5 is the most attractive general-purpose heuristic for mitigating the effects of the startup problem evaluated in this research. It is the most sensitive rule in detecting bias and the most consistent rule in mitigating its effects.

This result was obtained based using models not considered in the current research. Future research should attempt to reproduce the results reported in the literature using the methods developed in this research. Future research should also test MSER performance on additional, more complex processes models, such as SS7 model examined by Law. The MSER laboratory, we hope, will facilitate these future research efforts. 


\section{References}

Applied Material, Introduction of Automod, accessed Aug. 19, 2016, http://www.appliedmaterials.com/global-services/automation-software/automod

Arena Simulation Software, What is Simulation Software?, accessed Aug. 19, 2016, https://www.arenasimulation.com/

Banks, J., (Editor), 1998, Handbook of Simulation: Principles, Methodology, Advances, Applications, and Practice, Wiley

Bertoli, M., G. Casale, and G. Serazzi, 2007, The JMT Simulator for Performance Evaluation of NonProduct-Form Queueing Networks. Proceedings of the 40thAnnual Simulation Symposium, pp. 3-10

Bertoli, M., G. Casale, and G. Serazzi, 2009, JMT: Performance Engineering Tools for System Modeling. ACM Metrics Performance Evaluation Review, 36(4)10-15.

Billingsley, P., 1968, Convergence of Probability Measures. Wiley, New York.

Blomqvist, N., 1967, The Covariance Function of the M/G/1 Queueing system. Skandinavisk Aktuarietidskrift, 6:157-174

Cash, C. R., D. G. Dippold, J. M. Long, B. L. Nelson, and W. P. Pollard., 1992, Evaluation of tests for initial condition bias. In Proceedings of the 1992 Winter Simulation Conference, ed. J. J. Swain, D. Goldsman, R. C. Crain, and J. R. Wilson, Institute of Electrical and Electronics Engineers, Piscataway, NJ, 577-585

Cobb, M. J., 2000, Eliminating Initialization Bias while Controlling Simulation Run Length, Master Thesis, Department of Systems and Information Engineering, University of Virginia, Charlottesville, Virginia

Cohen, J. W., 1982, The Single Server Queue. North- Holland, Amsterdam

Conway, R. W., 1963, Some Tactical Problems in Digital Simulation, Management Science, 10(1)47-61

CreateaSoft, SimCAD Process Simulator, accessed Aug. 20, 2016, https://www.createasoft.com/

Emshoff, J. R., and R. L. Sission, 1970, Design and Use of Computer Simulation Models, Macmillan, New York

Fishman, G.S., 1971, Estimating Sampling Size in Computing Simulation Experiments, Management Science, 18(1)21-38

Fishman, G.S., 1973, Statistical analysis for queueing simulation, Management Science, 20:363-369

Fishman, G.S., 2001, Discrete-Event Simulation: Modeling, Programming, and Analysis, Springer-Verlag, New York

Franklin, W. W., and K. P. White, Jr., 2008, Stationarity Tests and MSER-5: Exploring the Intuition Behind Mean-Squared-Error-Reduction in Detecting and Correcting Initialization Bias, Proceedings of the 2008 Winter Simulation Conference, 541-546 
Franklin, W. W., K. P. White, Jr., and K. A. Hoad, 2009, Comparing Warm-up Methods using Mean-Squared Error, Working paper, Department of Systems and Information, University of Virginia, Charlottesville, VA. Available from the authors.

Franklin, W.W., 2009, The Theoretical Foundation of the MSER Algorithm, Ph.D. Dissertation, Department of Systems and Information Engineering, University of Virginia, Charlottesville, VA

Goldsman, D. and M.S. Meketon, 1986, A Comparison of Several Variance Estimators, Tech. Report \#J-85-12, School of ISyE, Georgia Tech, Atlanta.

Gordon, G., 1969, System Simulation, Prentice Hall PTR, Upper Saddle River, NJ

Grassmann, W.K., 2009, When, and when not to Use Warm-up Periods in Discrete Event Simulation. SimuTools 2009, Rome, 1-6

Grassmann, W.K., 2011, Rethinking the Initialization Bias Problem in Steady-State Discrete Event Simulation, Proceedings of the 2011 Winter Simulation Conference, 593-599

Hoad, K., and S. Robinson, 2011, Implementing Mser-5 in Commercial Simulation Software and Its Wider Implications, Proceedings of the 2011 Winter Simulation Conference, pp.495-503

Hoad, K., Robinson, S., and Davies, R., 2008, Automating Warm-up Length Estimation, Proceedings of the 2008 Winter Simulation Conference, 532-540

Hoad, K., Robinson. S., and Davies, R., 2011, AutoSimOA: a framework for automated analysis of simulation output, Journal of Simulation 5:9-24

Imagine That, Introduction of ExtendSim, accessed Aug. 20, 2016, http://www.extendsim.com/

Kelton, W. D., and A. M. Law, 1983, A New Approach for Dealing with the Startup Problem in Discrete Event Simulation, Naval Research Logistics Quarterly 30, pp. 641-658

Kelton, W. D., R. P. Sadowski, and N. Swets, 2010, Simulation with Arena, 5th edition, McGraw-Hill, New York

Krahl, D., 2012, ExtendSim: a history of innovation, Proceedings of the Winter Simulation Conference.

Lada, E. K., and J. R. Wilson, 2008, SBatch: A spaced batch means procedure for steadystate simulation analysis. Journal of Simulation 2:170-185.

Lada, E.K., 2003, A wavelet-based procedure for steady-state simulation output analysis, Ph.D Dissertation, available online via www.lib.ncsu.edu/theses/available/etd04032003-141616/unrestricted/etd.pdf

Law, M. A., 2015, Simulation Modeling and Analysis, 5th edition, McGraw-Hill, New York

Mahajan, P. S., and R.G. Ingalls, 2004, Evaluation of methods used to detect warm-up period in steady state simulation, Proceedings of the 2004 Winter Simulation Conference, pp. 663-671

McClarnon, M. A., 1990, Detection of steady state in discrete event dynamic systems: An analysis of heuristics. M.S. thesis, School of Engineering and Applied Science, 
University of Virginia, Charlottesville, Virginia. Available from University of Viginia Library, lib-lend@virginia.edu, 434-982-3094.

Meketon, M. S., and Schmeiser, B. W., 1984, Overlapping batch means: Something for nothing? Proceedings of the 1984 Winter Simulation Conference, 227-230.

Mokashi, A. C., J. J. Tejada, S. Yousefi, T. Xu, J. R. Wilson, A. Tafazzoli, and N. M. Steigher, 2010, Performance Comparison of MSER-5 and N-Skart on the Simulation Start-up Problem, Proceedings of the 2010 Winter Simulation Conference, pp.971982

Morse, P. M., 1955, Stochastic properties of waiting lines. Operations Research, 3(3) pp.1255-1261

Nelson, B.L., 2011, Thirty Years of Batch Size Effects, Proceedings in the 2011 Winter Simulation Conference, 392-400

Nelson, B. 2013, Foundations and Methods of Stochastic Simulation: A First Course. Springer Science, New York.

Pasupathy, R. and B. Schmeiser, 2010, The Initial Transient in State-State Point Estimation: Contexts, A Bibliography, The MSE Criterion, and The MSER Statistic, Proceedings of the 2010 Winter Simulation Conference, 184-197

Pasupathy, R., and B. Schmeiser. 2014. "MSER: Algorithms for the Initial-Transient Problem." Presented at the INFORMS Annual Meeting, 12 November

Pawliskowski, K., 1990, Steady-State Simulation of Queuing Processes: A Survey of Problems and Solutions, ACM Computing Surveys, 22(2)123-170

Peng, L., and Q. Yao, 2003, Least Absolute Deviation Estimation for ARCH and GARCH models, Biometria 90(4)967-975

Pollard, D., 1991, Asymptotics for least absolute deviation regression estimators, Econometric Theory 7:186-199

ProModel, 2011, ProModel tutorial accessed via https://www.promodel.com/solutionscafe/webinars/ProModel\%202011\%20Tutorial /PM2011Tutorial.html

Rossetti, M. D., P. J. Delaney, and K. P. White, Jr. 1995, Generalizing the half-width minimization heuristic for mitigating initialization bias, In Proceedings of International Conference on Systems, Man, and Cybernetics. Piscataway, New Jersey: Institute of Electrical and Electronics Engineers, Inc. pp. 212-216

Sanchez, P., and K.P. White, Jr. 2011, Interval Estimation Using Replication/Deletion and MSER Truncation, Proceedings of the 2011 Winter Simulation Conference, 488-494

Schmeiser, B., 1982, Batch size effects in the analysis of simulation output, Operations Research 30:556-568

Schmeiser. B., and W.T. Song., 1987, Correlation among estimators of the variance of the sample mean. Proceedings of the Winter Simulation Conference, pp.309-317

Schruben, L.W., 1982, Detecting Initialization Bias in Simulation Output, Operation Research 30(3)569-590

Schruben, L. W., 1983, Confidence interval estimation using standardized time series. Operations research, 30:1090-1108 
Simio Release 2 - Sprint 41 - July 21, 2010 to implement the research logic, accessed Aug. 20, 2016, http://www.simio.com/products/ and Simio Reference Guide

SIMUL8 Software, Simul8 Introduction, accessed Aug. 19, 2016, http://www.simul8.com/

Solomon, S. L., 1983, Simulation of Waiting-Line Systems. Prentice-Hall, Englewood Cliffs, N.J.

Song, W. T., 1996, On the Estimation of Optimal Batch Sizes in the Analysis of Simulation Output, European Journal of Operational Research, 88:304-319

Song, W. T., and B. Schmeiser, 1993, Variance of the Sample Mean: Properties and Graphs of Quadratic-Form Estimators, Operations Research 41:501-517

Song, W.T., and B. Schmeiser, 1995, Optimal Mean-Squared-Error Batch Sizes, Management Science 41:110-123.

Spratt, S. C., 1998, An Evaluation of Heuristics for the Startup Problem

Tafazzoli, A., 2009, Skart: A skewness- and autoregression-adjusted batch-means procedure for simulation analysis. PhD Dissertation, Department of Industrial and Systems Engineering, North Carolina State University, Raleigh, North Carolina. Available via www.lib.ncsu.edu/theses/available/etd-01122009153054/unrestricted/etd.pdf [accessed March 3, 2012].

Tafazzoli, A., and J. R. Wilson, 2009, N-Skart: A Nonsequential Skewness-and Autoregression-adjusted Batch-means Procedure for Simulation Analysis. Proceedings of the 2009 Winter Simulation Conference, pp.652-662

Tocher, K. D.,1963, The Art of Simulation, English Universities Press

Turnquist, M. A., and J. M. Sussman, 1977, Toward Guidelines for Designing

Experiments in Queuing Simulation. Simulation, 28:137-144

Wang, R. J., and P. W. Glynn, 2014, On the Marginal Standard Error Rule and the Testing of Initial Transient Deletion Methods, working paper, Department of Management Science and Engineering, Stanford University. Stanford, CA. Accessed 30 April 2015. http://web.stanford.edu/ glynn/papers/2014/WangG14.pdf

Welch, P. D., 1981, On the Problem of the Initial Transient in Steady-State Simulation, IBM Watson Research Center, Yorktown Heights, New York.

White, K. P., Jr. 1995, A simple rule for mitigating initialization bias in simulation output: Comparative results. In Proceedings of the International Conference on Systems, Man, and Cybernetics, Piscataway, New Jersey: Institute of Electrical and Electronics Engineers, Inc., pp. 206-211.

White, K.P. Jr., 1997, An Effective Truncation Heuristic for Bias Reduction in Simulation Output, Simulation 69(6),323-334

White, K. P., Jr., 2012, Optimal analysis for the mean of a simulation output, Department of Systems and Information Engineering, University of Virginia, working paper

White, K. P. Jr., M. J. Cobb, and S. C. Spratt, 2000, A Comparison of Five Steady-State Truncation Heuristics for Simulation, Proceedings of the 2000 Winter Simulation Conference, pp.755-760 
White, K. P. Jr., and W. W. Franklin, 2010, Parametric Expression For MSER with Geometrically Decaying Bias, Proceedings of the 2010 Winter Simulation Conference, 957- 964

White, K. P., Jr., and Hwang, S. N., 2015, Delay times in an M/M/1 queue: estimating the sampling distribution for the MSER-truncated steady-state mean using replication/deletion, Proceedings of the 2015 Winter Simulation Conference, pp. 493-504

White, K. P., and M. A. Minnox, 1994, Minimizing initialization bias in simulation output using a simple heuristic. In Proceedings of International Conference on Systems, Man, and Cybernetics, Piscataway, New Jersey: Institute of Electrical and Electronics Engineers, Inc., pp. 215-220.

White, K. P., Jr., and S. Robinson, 2010, The initial transient problem (again), or why MSER works, Journal of Simulation, 4(3)268-272

Wilson, J. R., and A. A. B. Pritsker, 1978, Evaluation of startup policies in simulation experiments. Simulation, 31:79-89

Yousefi, S., 2011, MSER-5Y: An Improved Version of MSER-5 with Automatic Confidence Interval Estimation, Master thesis, North Carolina State University, Raleigh, NC 


\section{Appendix I. Arena MSER Submodel User Guide}

This appendix provides guidance that will allow you to incorporate the purpose-built MSER submodel in your Arena simulation model.

\section{Variable definition}

After building a model, you can choose entity's attribute(s) of interest that are passed to a Arena MSER submodel.

1. General simulation variables:

a. X: Attribute of interest (i.e., entity waiting time)

b. StopRule: User input to end simulation

c. Counter: Number of entities

2. MSER related variables: You need to define the following additional variables to store temporary values and compute MSER statistics associated with each entity.

a. Truncation: Truncation index

b. MSER_test: MSER statistics without truncation

c. MSER_final: MSER statistics related to each truncation index

d. X2: Squared values of attribute $X$

e. inter_cumX: Summation of X associated with each entity

f. inter_cumX2: Summation of X2 associated with each entity

g. cumX: Storage for summation of $X$ associated with each entity

h. cumX2: Storage for summation of X associated with each entity

i. Mean: Average value from the first $\mathrm{X}$ to the current one by dividing cumX by Counter

To avoid confusion, all of variables are preceded with " $v_{-}$" inside the Arena submodel with the exception StopRule. Equations $2 b$ and $2 c$ are used to compute MSER statistic.

$2 b$ :

$$
\left(\sum_{i=1}^{\text {Counter }} X_{i}^{2}-\text { Counter } \cdot \sum_{i=1}^{\text {Counter }} X_{i} \cdot \sum_{i=1}^{\text {Counter }} X_{i}\right) /((\text { Counter }-0) \cdot(\text { Counter }-0))
$$

2c:

$\left(\sum_{i=1}^{\text {StopRule }} X_{i}^{2}-\sum_{i=1}^{\text {truncation }} X_{i}^{2}-(\right.$ StopRule - Truncation $\left.) \cdot \sum_{i=1}^{\text {StopRule }} X_{i} \cdot \sum_{i=1}^{\text {StopRule }} X_{i}\right) /(($ StopRule-Truncation $) \cdot($ StopRule-Truncation-1 $))$ 
All required variables are listed in Table I.1. For example, if you were interested in entity wait time, choose its wait time attribute as a global variable, $v_{-} X$. In this table, the "Location of block" column indicates where the corresponding variables are located in building blocks in Arena model.

Table I.1. Global, Local variables and Arrays

\begin{tabular}{|c|c|c|c|}
\hline No. & $\begin{array}{l}\text { Variable } \\
\text { name }\end{array}$ & Location of block & Usage \\
\hline 1 & v_Counter & Index & $\begin{array}{l}\text { Record entity number } \\
\text { v_Counter }+1\end{array}$ \\
\hline 2 & v_X & $\begin{array}{l}\text { InterimData } \\
\text { Generation }\end{array}$ & Record entity wait time \\
\hline 3 & $\mathrm{v} \_\mathrm{X} 2$ & $\begin{array}{l}\text { InterimData } \\
\text { Generation }\end{array}$ & Record (entity waiting time) ${ }^{2}$ \\
\hline 4 & v_inter_cumX & $\begin{array}{l}\text { InterimData } \\
\text { Generation }\end{array}$ & Holder for summation of $v_{-} X$ \\
\hline 5 & v_inter_cumX2 & InterimData & Holder for summation of $\mathrm{v} \_\mathrm{X} 2$ \\
\hline 6 & v_Mean & DataGeneration & v_cumX(v_Counter $) / v_{-}$CCounter \\
\hline 7 & $\mathrm{v}_{-}$cumX & DataGeneration & v_inter_cumX(v_counter) \\
\hline 8 & $\mathrm{v}_{-}$cumX2 & DataGeneration & v_inter_cumX2(v_counter) \\
\hline 9 & v_MSER_test & $\begin{array}{l}\text { MSER Without } \\
\text { Truncation }\end{array}$ & 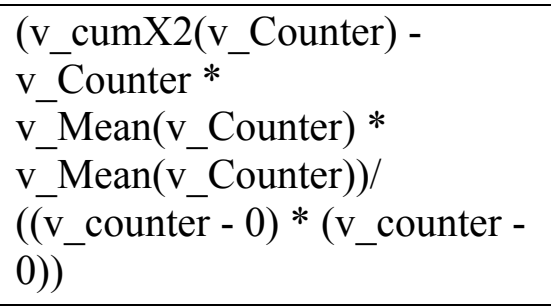 \\
\hline 10 & StopRule & $\begin{array}{l}\text { End of Simulation } \\
\text { (Decision block) }\end{array}$ & $\begin{array}{l}\text { Global variable to check the } \\
\text { end of simulation, } 10000 \text { that } \\
\text { confirm } \\
\text { StopRule = v_Counter }\end{array}$ \\
\hline 11 & v_Truncation & Truncation Index & $\mathrm{v}_{-}$Truncation +1 \\
\hline 12 & v_MSER_final & $\begin{array}{l}\text { MSER } \\
\text { With Truncation }\end{array}$ & 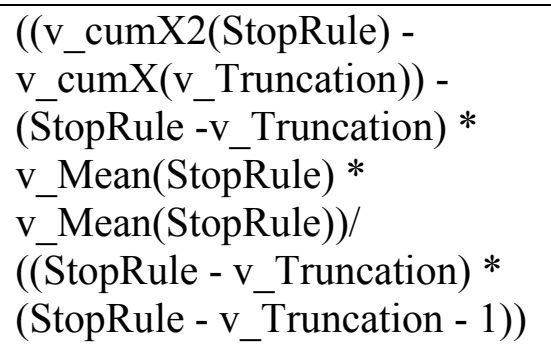 \\
\hline
\end{tabular}




\section{Run length control}

As shown in Figure I.1, the global variable StopRule must be defined before running a model. After assign a value to StopRule, such as 10000 entities, you need to use this variable as the terminating condition on the Replication Parameters tab of the Arena Run Setup menu.

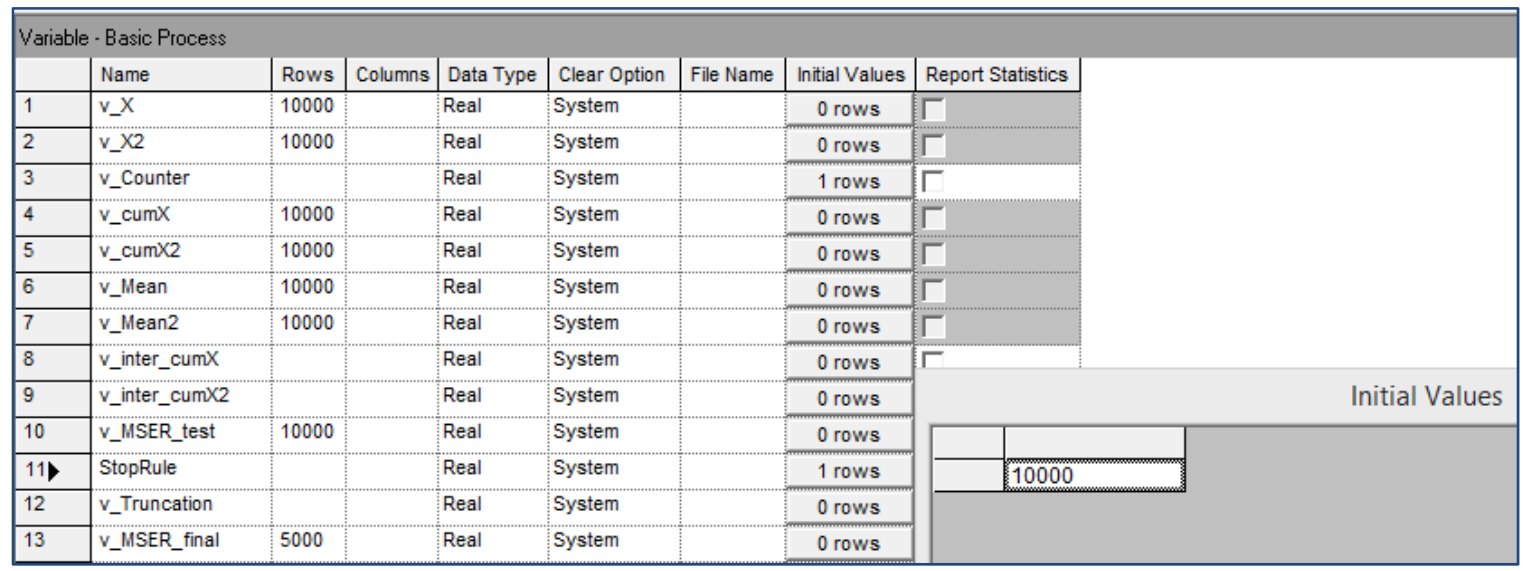

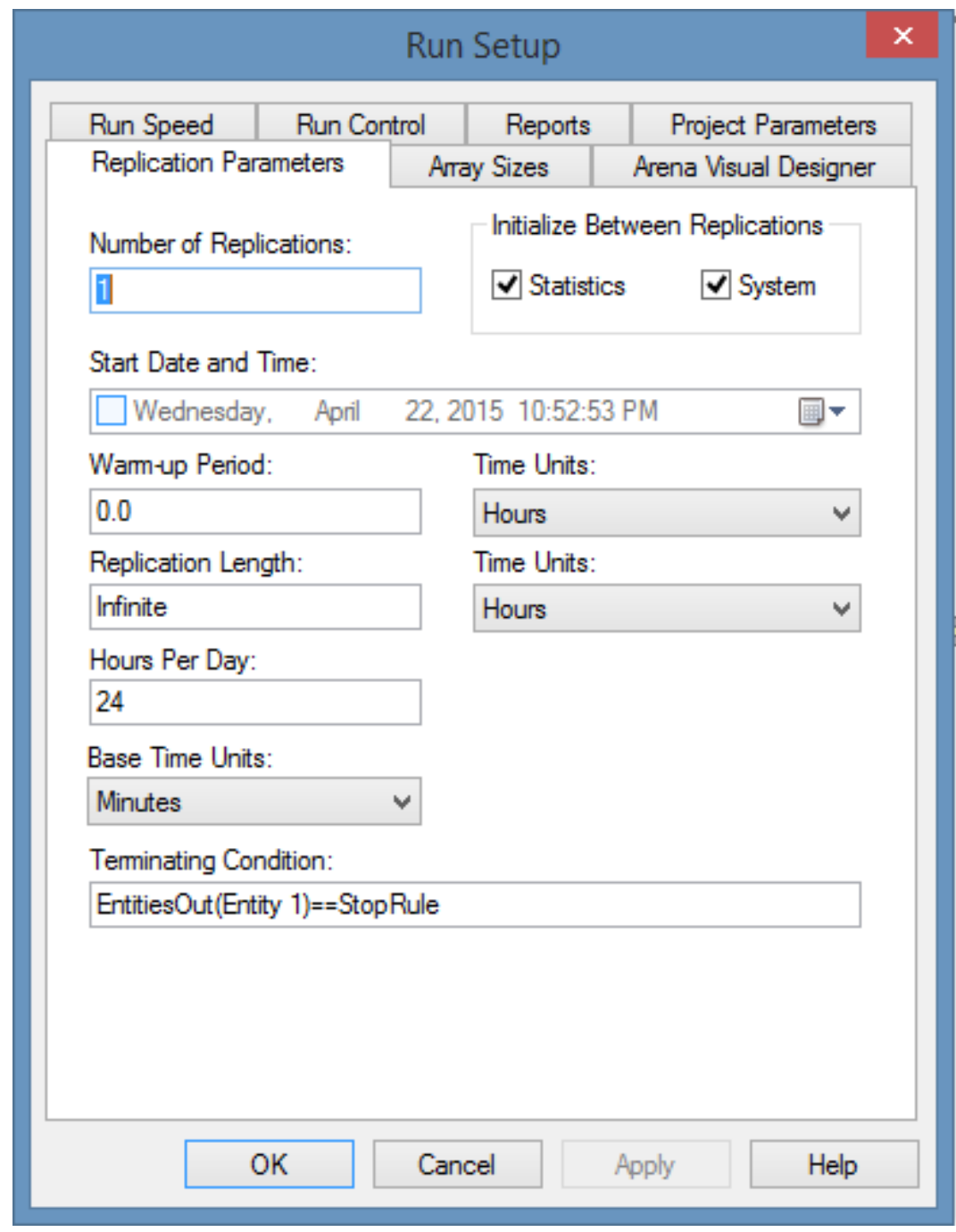

Figure I.1. Setting the Simulation Length using the variable StopRule. 


\section{Usage of MSER Submodel}

As an example, consider the case where the output of interest is the mean entity waitingtime for an $\mathrm{M} / \mathrm{M} / 1$ queue in steady state. When building the model, include the MSER submodel as shown in Figure I.2.

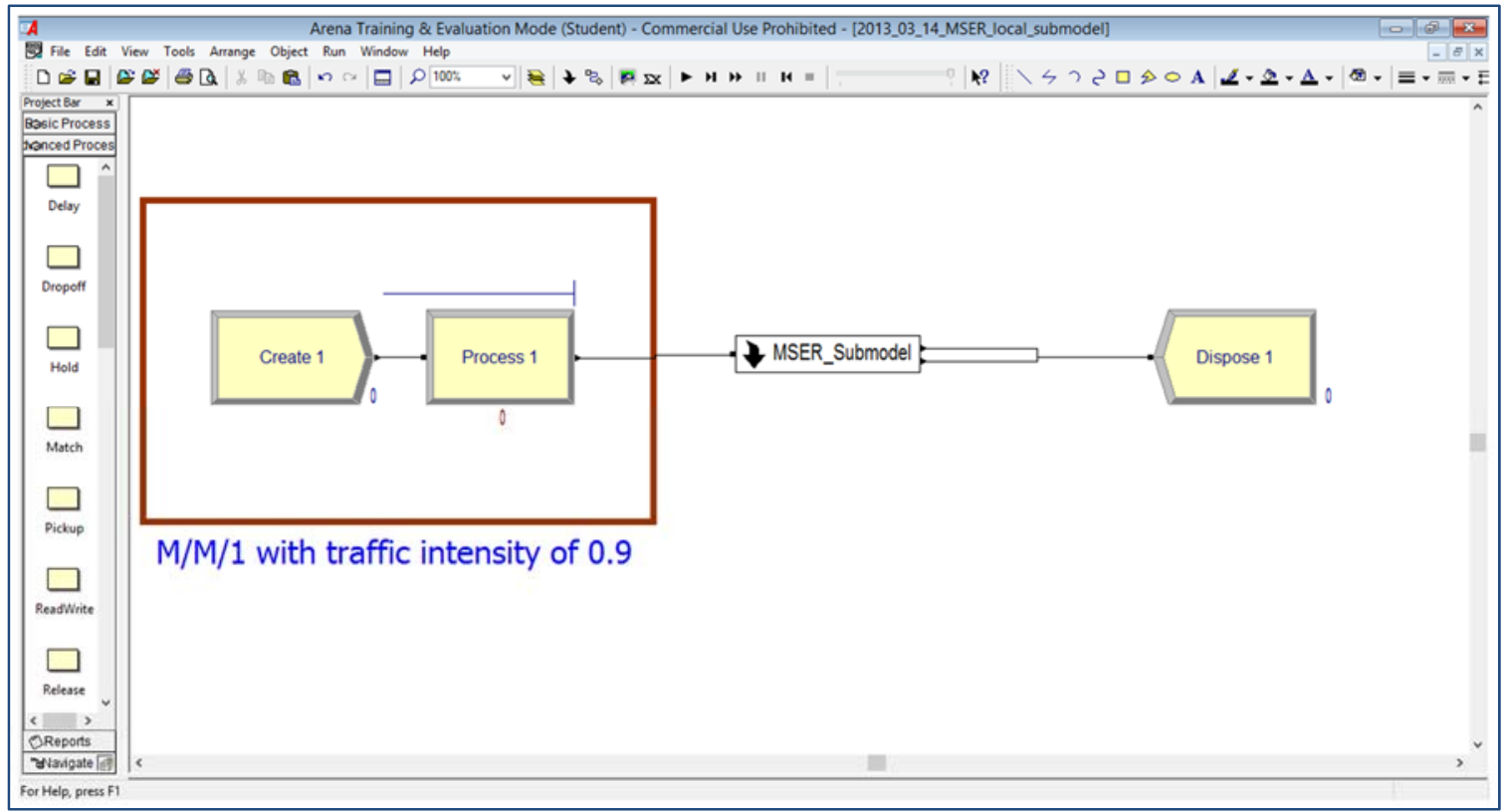

Figure I.2. Main model for the example.

As shown in Figure I.3, the MSER submodel consists of three major parts: (1) Data preprocessing, (2) MSER test generation, and (3) MSER statistics generation. The second part is included simply to test whether or not the calculations are performed correctly and can be ignored after completing the model. 


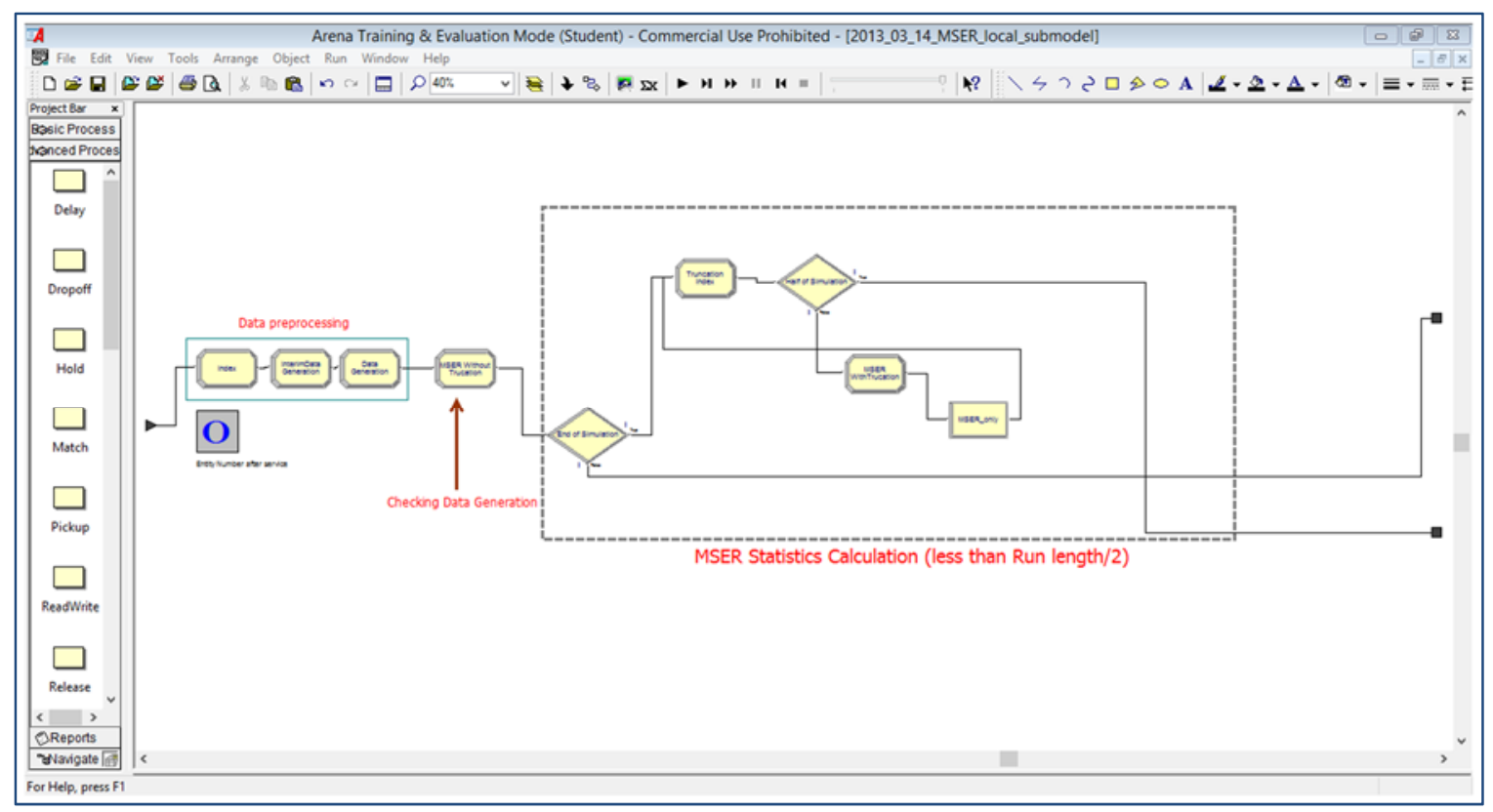

Figure I.3. Submodel to compute MSER.

Data preprocessing uses three Assign modules to define variables and variable arrays that reflect the previous variable definitions, as shown in Figure I.4.

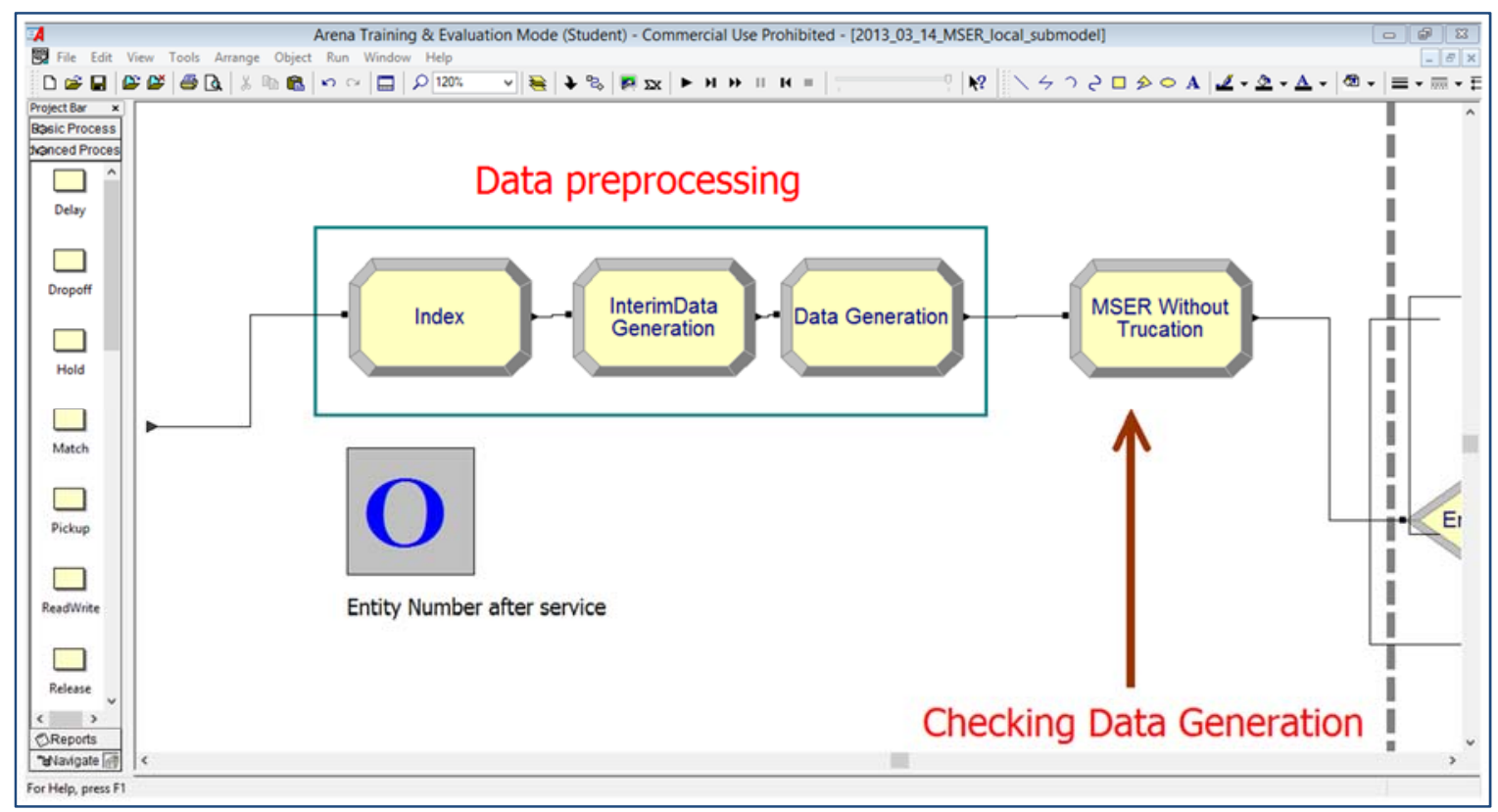

Figure I.4. Data preprocessing in Submodel.

The Index Assign module increments the variable v_Counter by 1 as each entity passes through the module, as shown in Figure I.5. This variable is used as an index into the arrays in the next Assign module. 


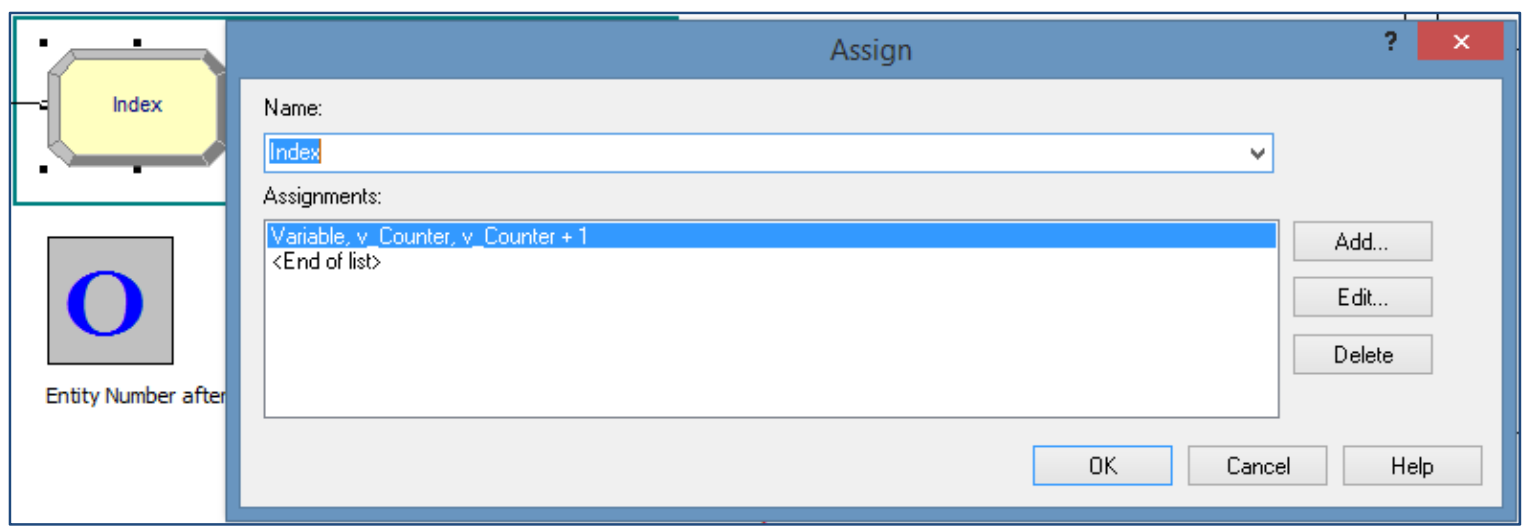

Figure I.5. Index incremented in an Assign module.

As shown in Figure I.6, the Interim Data Generation Assign module assigns values to the elements of two variable arrays, $\mathrm{X}$ and X2, using V_Counter as the index into these arrays. This module also assigns a value to the variable inter_cumX. These variables are subsequently used to update the MSER statistic.

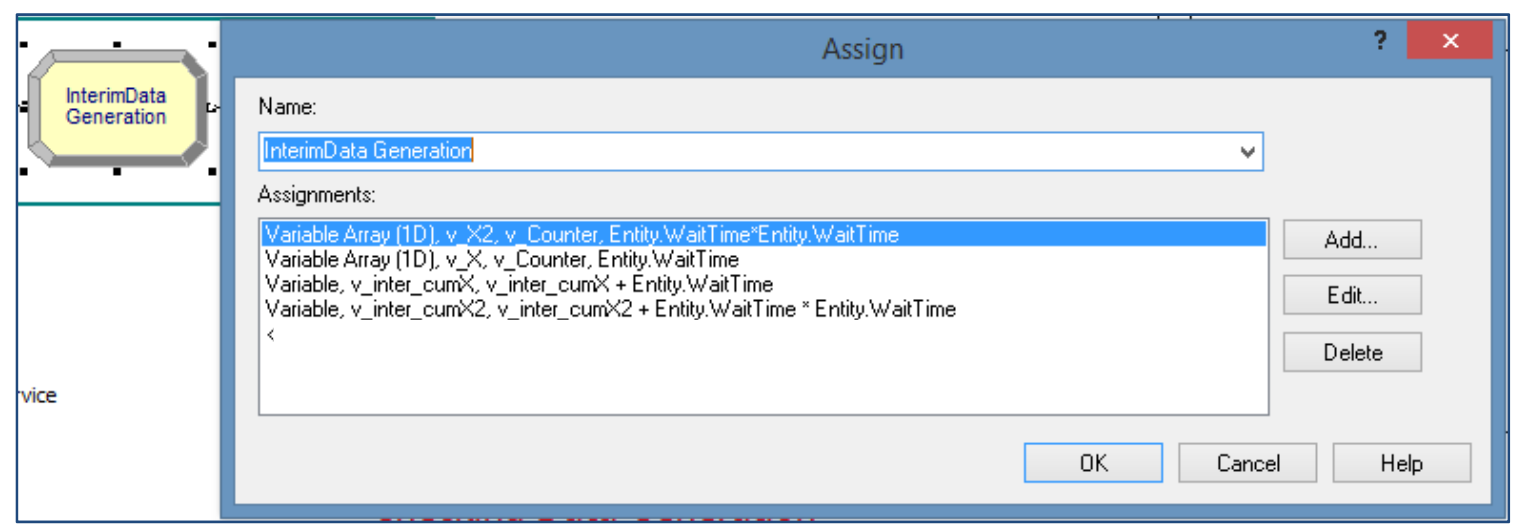

Figure I.6. Interim Data Generation Assignments.

As shown in Figure I.7, the Data Generation assignment module assigns three variable arrays that also used to update the MSER statistic. 


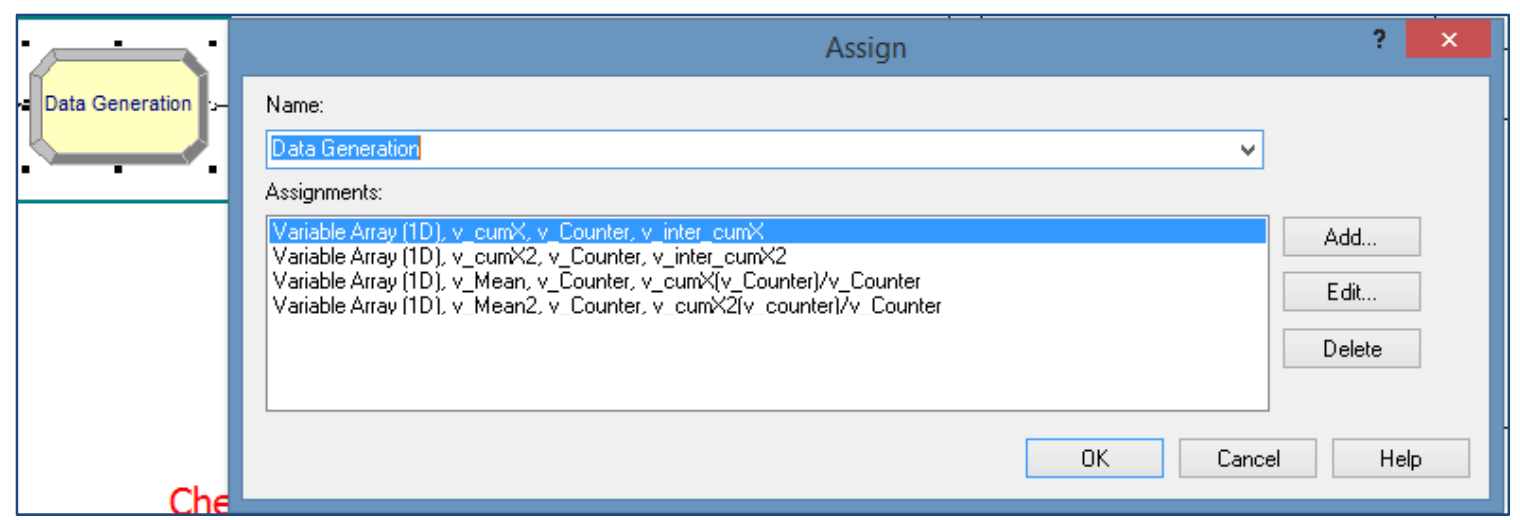

Figure I.7. Data Generation in Assignment.

As noted previously, this assignment shown in Figure I.8 is included to test whether or not the calculations are performed correctly and can be removed after performing the test satisfactorily.

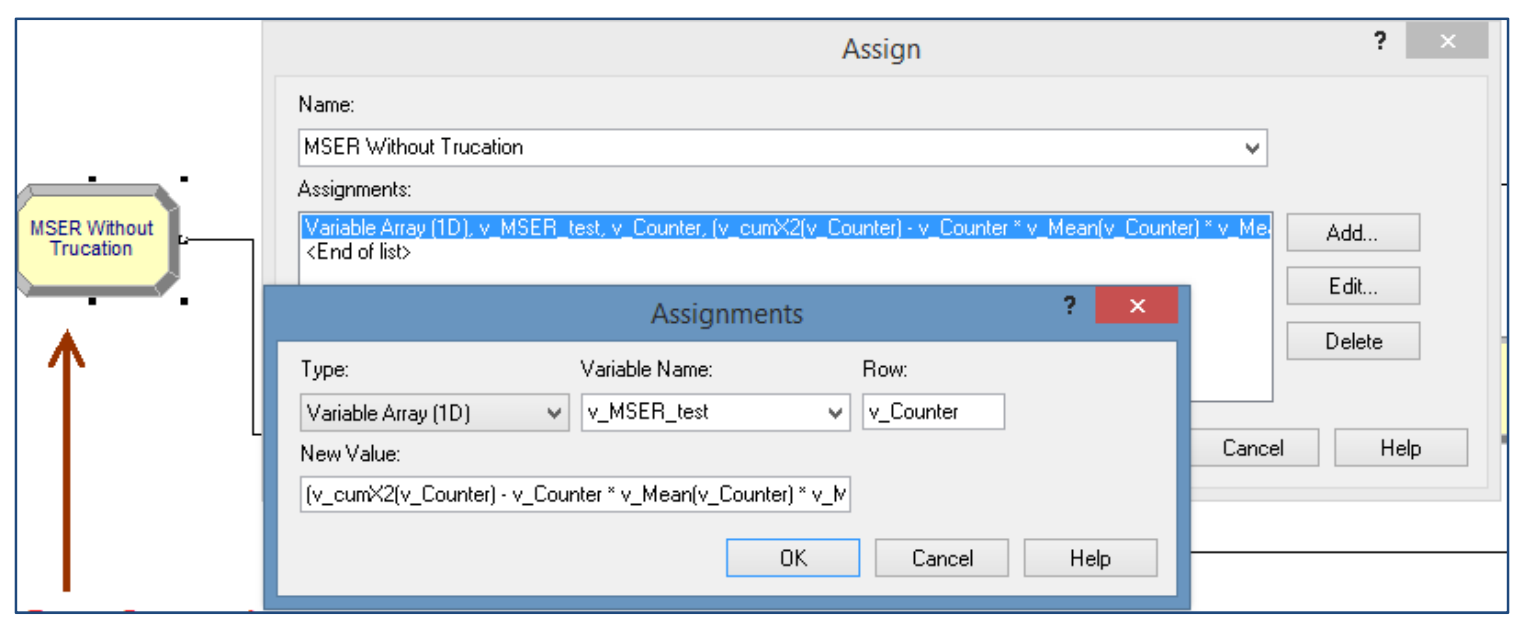

Figure I.8. MSER without Truncation in Assignment.

Figure I.9 shows the core building blocks used to compute MSER statistics. 


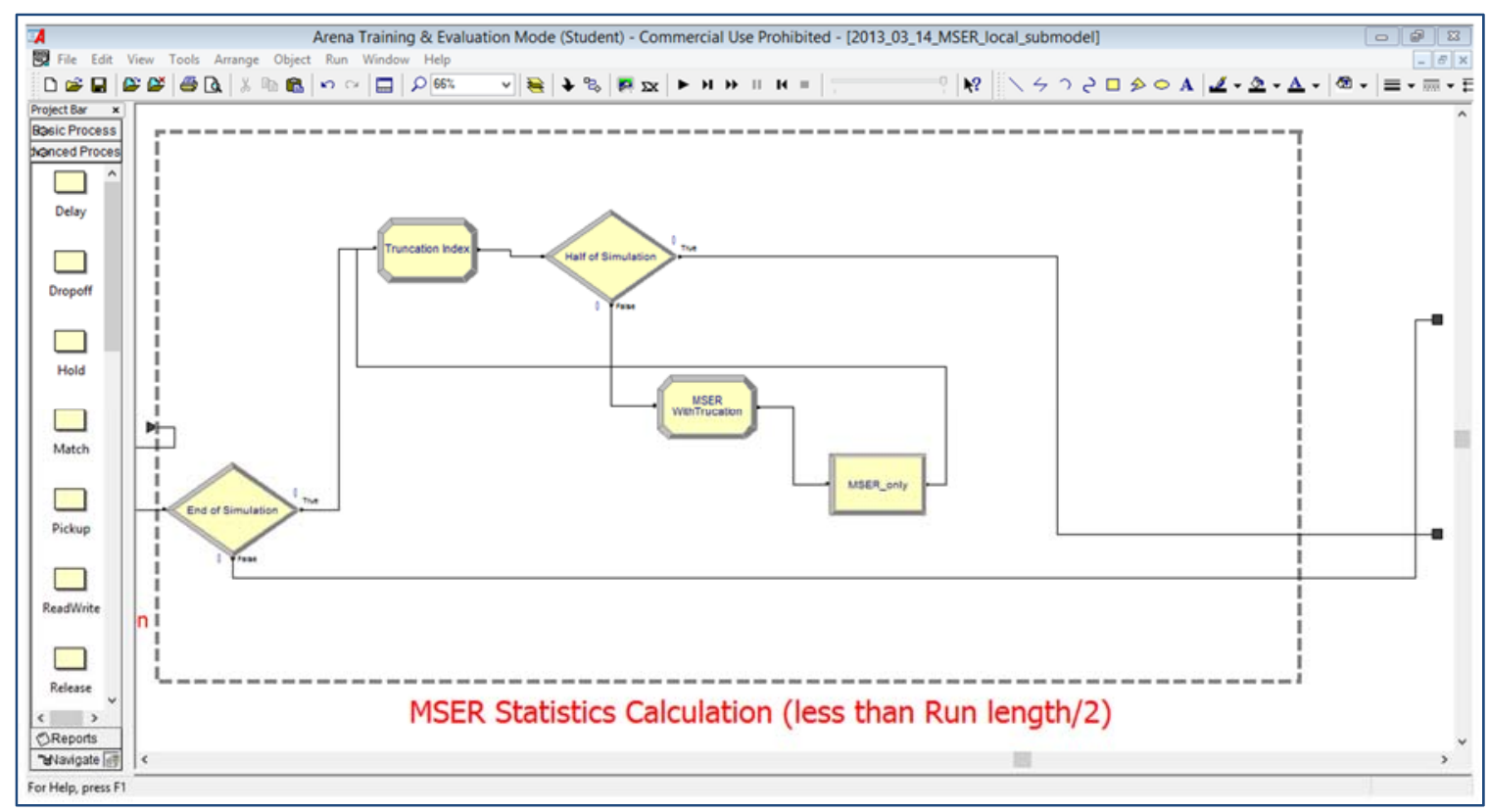

Figure I.9. MSER Statistics calculation in Submodel.

The decide module in Figure I.10 compares the value of the variable V_Counter to the value of the variable StopRule to determine whether or not the stopping condition has been met. If not, the entity exits the Arena MSER submodel.

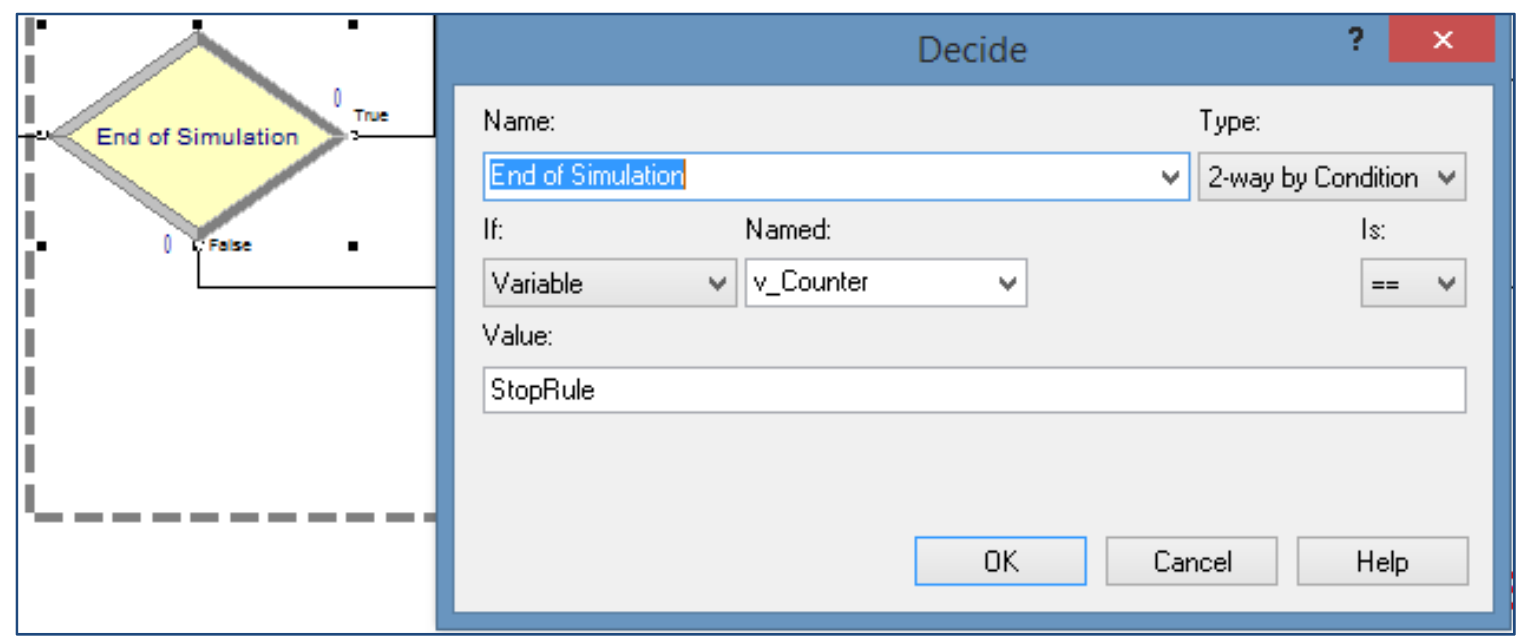

Figure I.10. End of Simulation in Decision Node.

Once the stopping condition is met, the entity proceeds to the Truncation Index assignment module shown in Figure I.11. Here the index variable v_Truncation is incremented by 1 . 


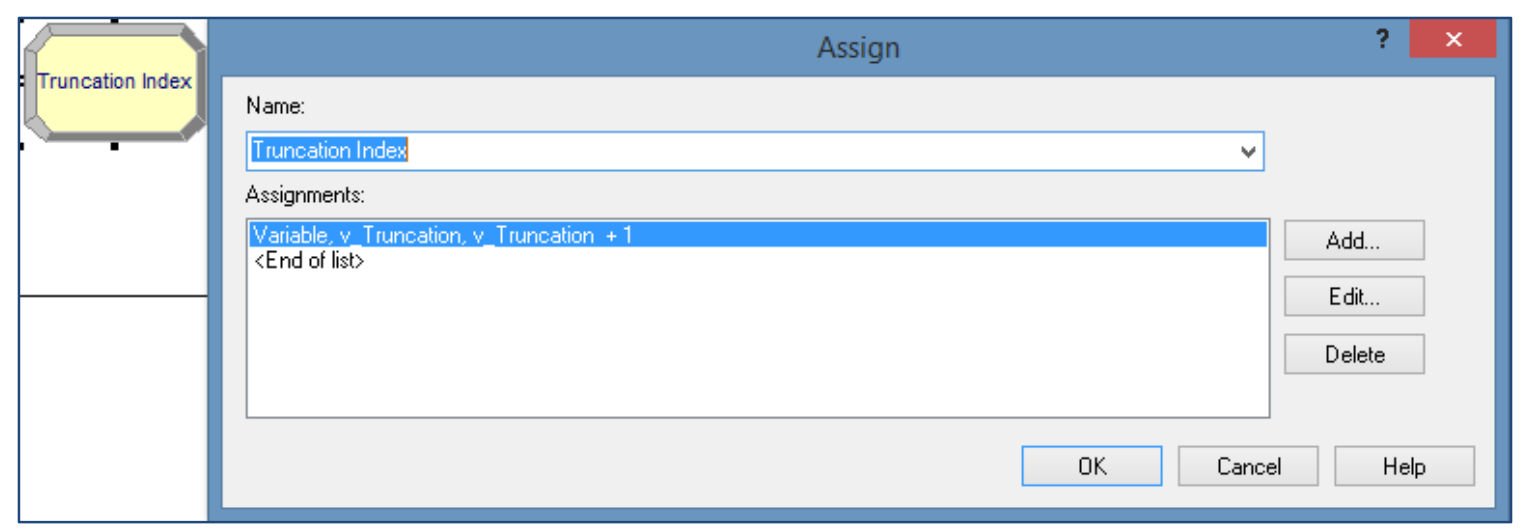

Figure I. 11 Truncation Index in Assignment.

This decision node shown in Figure I.12 checks whether or not the half of simulation run is completed.

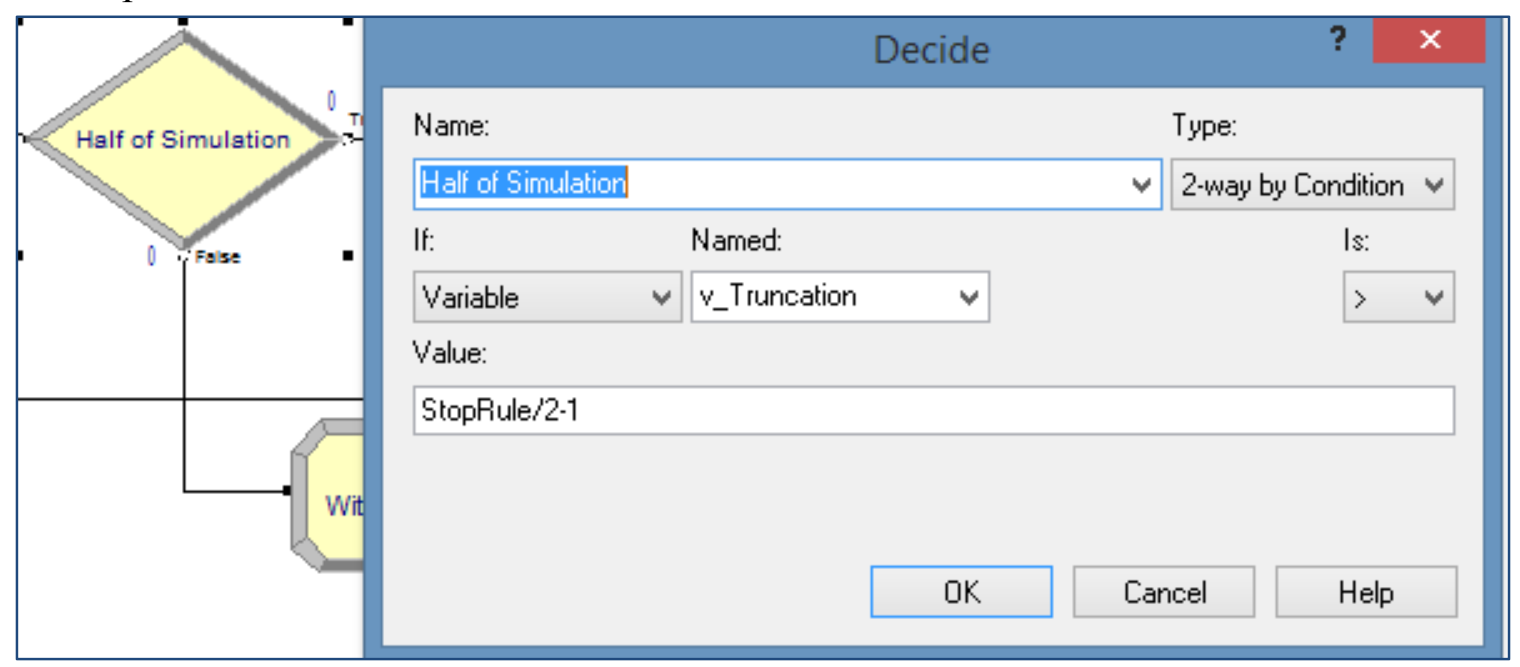

Figure I.12. Half of Simulation in Decision Node.

If not, then the MSER statistics are calculated in the MSER With Truncation Assign module as shown in Figure I.13. The logic in Equation 2c is incorporated in the New Value field for the current truncation index to assign the value calculated for the MSER statistic, an element of the global variable array v_MSER_final. 


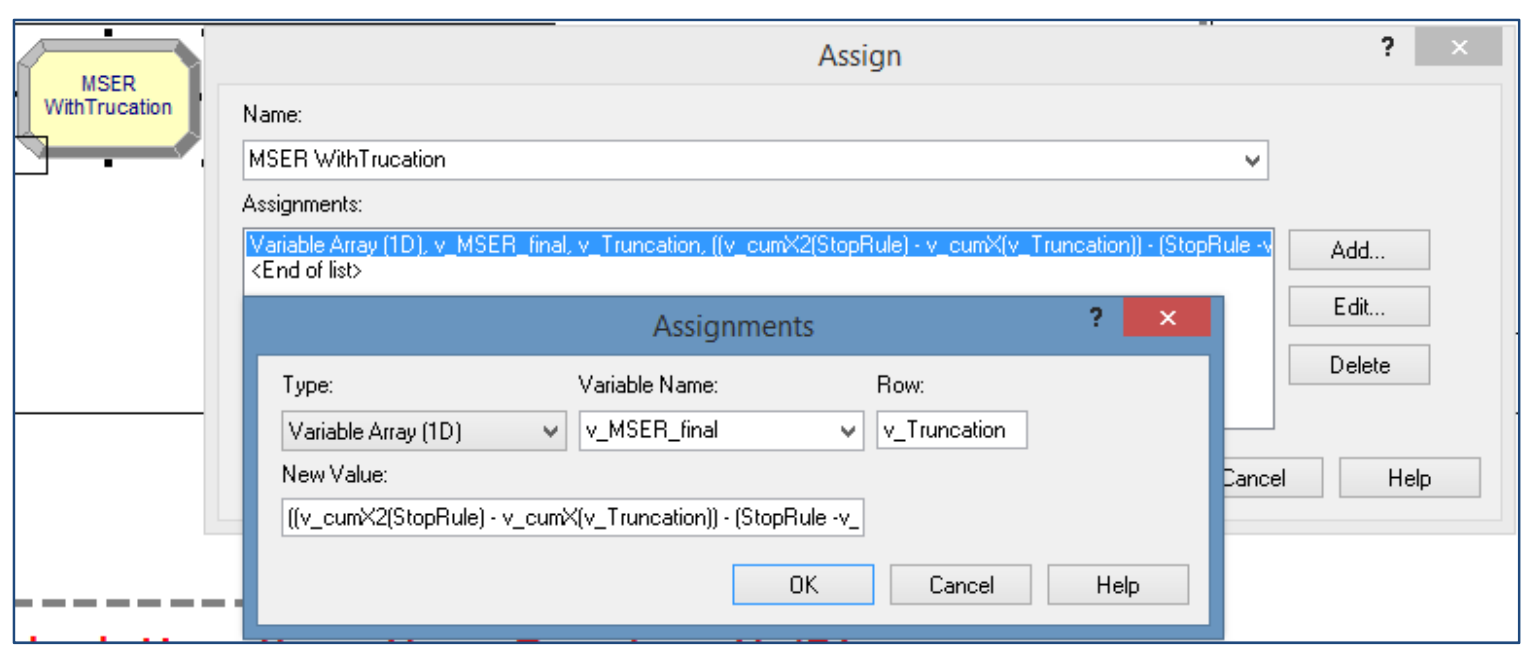

Figure I.13. MSER with Truncation in Assignment.

These values are written to an external file using a ReadWrite module shown in Figure I. 14 .

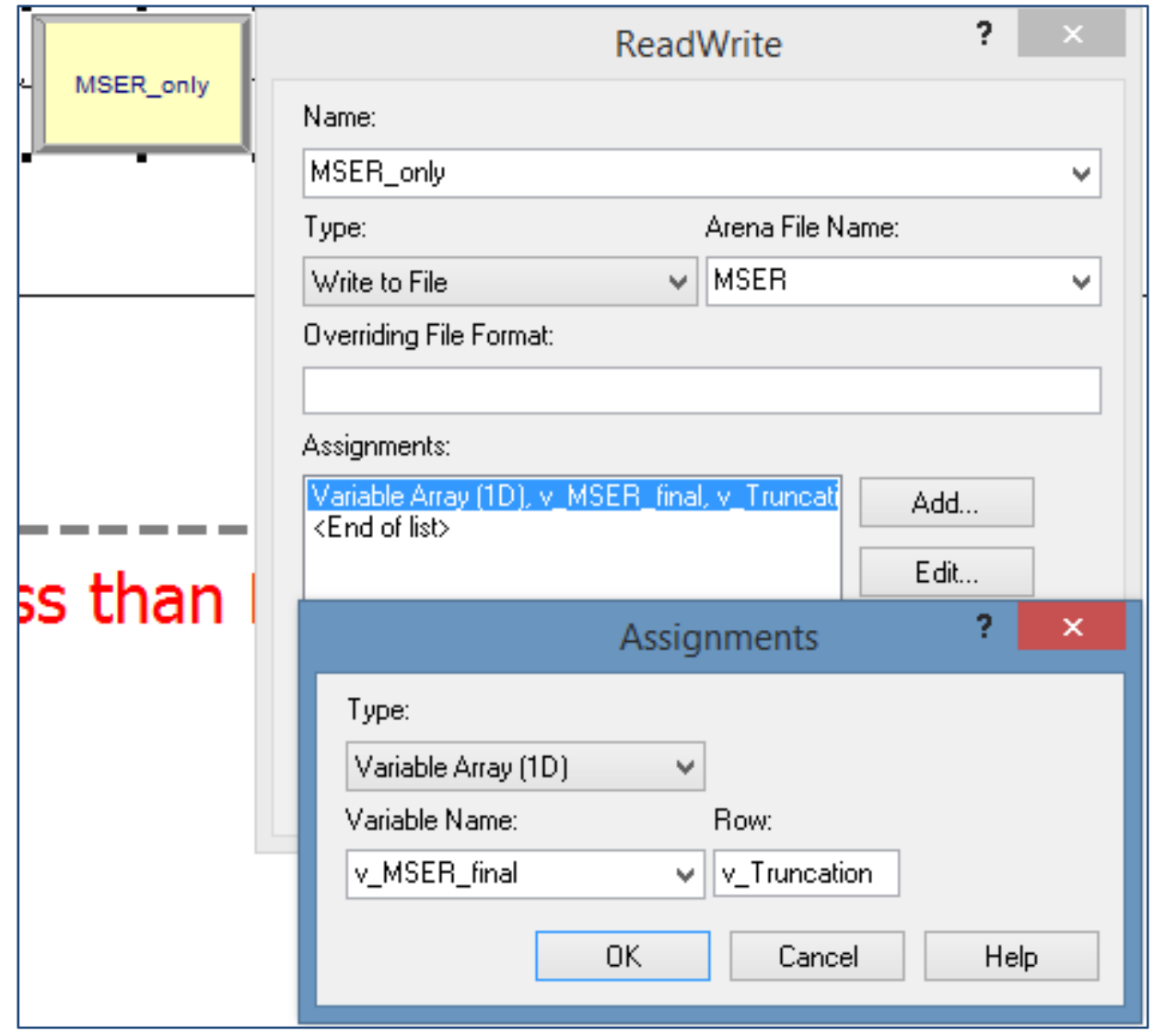

Figure I.14. MSER_only in ReadWrite.

The internal Arena File name used here is identified with an operating-system file using the File data module on the Advanced Process panel, as shown in Figure I.15. In this 
example, the operating-system file is named MSER_array.csv and located in the same folder in which the Arena model. The extension indicates this file is in csv format.

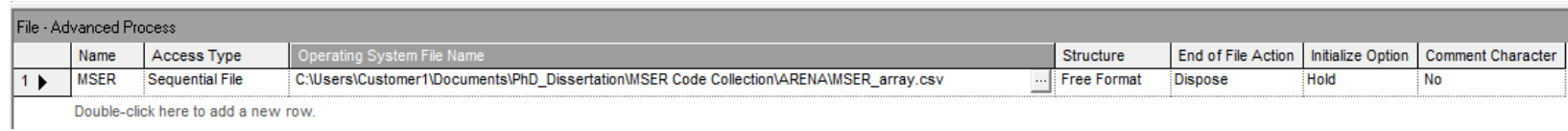

Figure I.15. Store MSER in File.

The file includes two columns, the first indicating the truncation index and the second the corresponding value of the MSER statistic. The minimum value of the MSER statistic in the second column is the optimal truncation point indicated by the corresponding truncation index. See Figure I.16 as an example.

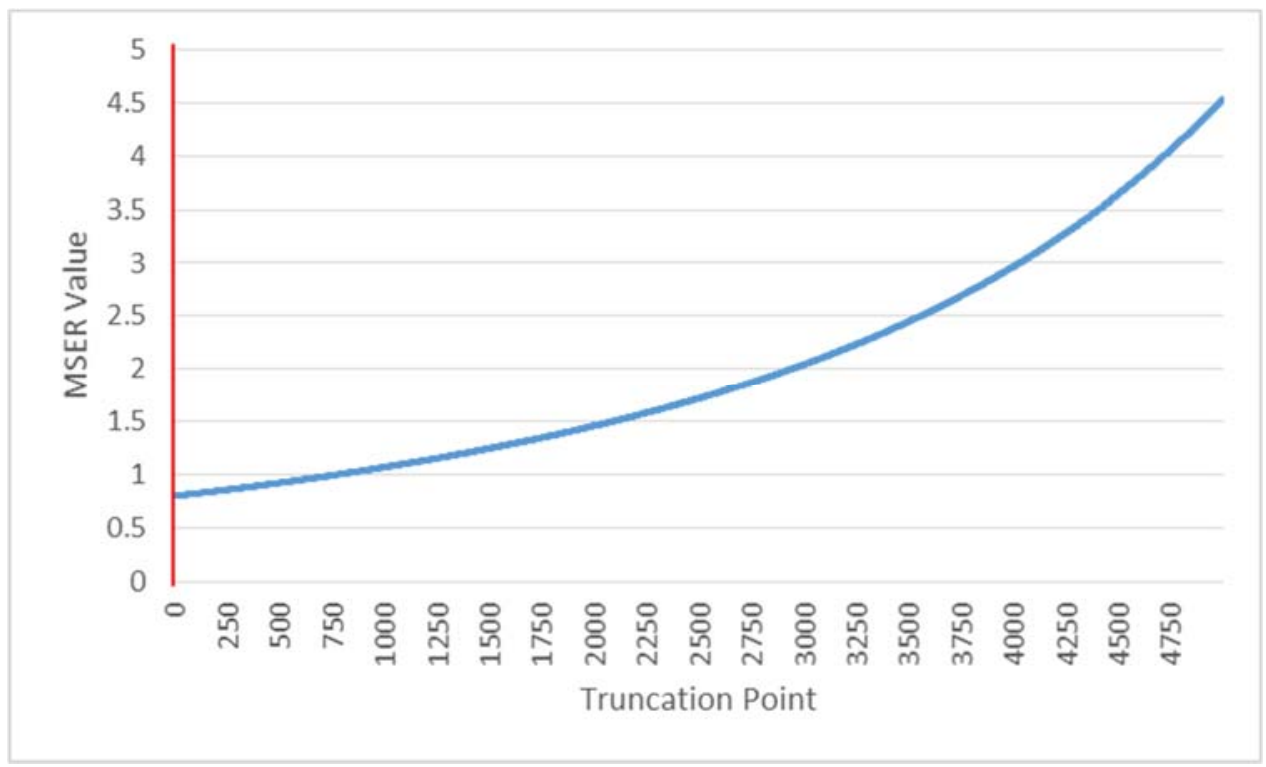

Figure I.16. MSER in File Representation (Optimal Truncation Point: Zero) 


\section{Appendix II. Personal reflections on the importance of the warm-up problem and undergraduate simulation curriculum survey}

\section{II.1 Anecdotal case to emphasize the importance of warm-up period}

Some of working professionals representing simulation specialists do not have in-depth knowledge and fundamental understanding of discrete event simulation. In fact, they are likely to build models based on a specific software environment. Thus, as long as its builtin functionalities are enough to reflect key characteristics of the system, only potential minor error might occur. For example, when a modeler sets up the travel distance of moving resources, she or he should acknowledge the behavior of returning resources imposed by some software application. However, we seldom see a simulation professional using warm-up periods with a solid understanding.

\section{II.2 Undergraduate curriculum survey}

We reviewed the twenty curricula or handbooks in industrial or systems engineering departments in the U.S. colleges. Thirteen out of twenty universities apparently mandate undergraduates to take credits of simulation course for graduation. Six other universities also describe simulation as a key methodology to learn before graduation. Even though one school does not have undergraduate programs, that school provides simulation courses for graduates. Most courses are 3 or 4 credits which are recommended to take during the third year. Table AII.1 summarizes detailed information about the survey.

This requirement apparently emphasizes the importance and the difficulty of simulation courses. However, this tendency does not guarantee those students taking simulation 
classes to be confident in building complex and sophisticated models. First, it only takes one semester to fulfill this credit. After finishing the course as a junior student, she or he might lose the insight and lessons learned over time.

Second, they might have to re-learn using other specific simulation package after their graduation if the worksite's tool is different from what they learned. Third, with respect to output analysis, its contents tend to be addressed near the end of the course. Sometimes, students are overwhelmed by the last minute pressures while instructors might not have enough time to provide in-depth lessons. These are the reasons that students and even experienced modelers do not fully appreciate the criticality of analyzing output statistics. Furthermore, most of simulation software applications provide 95 or $90 \%$ confidence interval by merely clicking an option.

Additionally, simulation software training courses from software vendors consist of two parts: Basic/introductory course and the following advanced course. The first course usually provides the brief review of simulation methodology and teaches how to build reasonable size models using built-in functions. Its major intents lie in familiarizing how to use that specific software. By completing this course, the course taker will likely know how to build relatively formulated models. However, they might fail to incorporate some key ingredients of a problem.

As the beginners and intermediate modelers encounter more complex situations, they will turn to their software vendors for additional support. These needs will urge them to take an advanced course to learn how to use optimization add-ins and to code inside or outside extensions. Both of these courses usually require two or three day boot camps, 


\section{TableAII.1 Twenty Undergraduate Curricula of Simulation}

\begin{tabular}{|c|c|c|c|c|}
\hline No. & School name & $\begin{array}{c}\text { Undergraduate } \\
\text { Mandatory }\end{array}$ & $\begin{array}{l}\text { No. of } \\
\text { Credit }\end{array}$ & Source \\
\hline 1 & Columbia University (Fu Foundation) & Yes & 4 & \\
\hline 2 & Cornell University & Yes & 4 & http://www.orie.cornell.edu/orie/academics/undergraduate/requirements.cfm \\
\hline 3 & Georgia Institute of Technology & Yes & 3 & http://www.isye.gatech.edu/academics/undergraduate/courses/ \\
\hline 4 & Lehigh University (Rossin) & Yes & 3 & http://www.lehigh.edu/ise/documents//E\%20Major\%20Requirements\%2009_10.pdf \\
\hline 5 & North Carolina State University & Yes & 3 & https://portalsp.acs.ncsu.edu/psp/EP91PRD/EMPLOYEE/PCS900PRD/c/NC_SSS_MENU.NC_AA_REQMNT_RPT.GBL \\
\hline 6 & Northwestern University (McCormick) & Yes & 3 & http://www.iems.northwestern.edu/docs/undergraduate/AY\%2013-14\%20BSIE\%20Degree\%20Requirements.pdf \\
\hline 7 & Purdue University-West Lafayette & Not clear & 3 & https://engineering.purdue.edu/IE/Academics/Undergrad/Curriculum \\
\hline 8 & Stanford University & Optional & 3 & http://exploredegrees.stanford.edu/schoolofengineering/managementscienceandengineering/\#bachelorstext \\
\hline 9 & Texas A\&M University-College Station (Look) & Not clear & 3 & http://engineering.tamu.edu/industrial/academics/courses/course-descriptions \\
\hline 10 & University of Arkansas-Fayetteville & Yes & 3 & http://www.ineg.uark.edu/Undergrad_Handbook_Spring_20140423.pdf \\
\hline 11 & University of California-Berkeley & Not clear & 3 & http://ieor.berkeley.edu/AcademicPrograms/Ugrad/Courses/index.htm \\
\hline 12 & University of California-Santa Barbara & Not clear & 3 & http://engineering.ucsb.edu/current_undergraduates/pdf/00-01Announce.pdf \\
\hline 13 & University of Florida & Yes & 3 & https://catalog.ufl.edu/ugrad/current/engineering/majors/industrial-and-systems-engineering.aspx \\
\hline 14 & University of Illinois-Urbana-Champaign & Yes & 3 & http://provost.illinois.edu/programsofstudy/2014/fall/programs/undergrad/engin/ind_engin.html \\
\hline 15 & University of Michigan-Ann Arbor & Yes & 4 & http://ioe.engin.umich.edu/degrees/ugrad/ugdocs/UndergradStudentGuide.2013.2014.pdf \\
\hline 16 & University of Pittsburgh (Swanson) & Yes & 3 & http://www.engineering.pitt.edu/Industrial/Undergraduate/Curriculum_Effective_as_of__all__2014/ \\
\hline 17 & University of Southern California (Viterbi) & Not clear & 3 & http://ise.usc.edu/academics/undergrad/undergrad.htm \\
\hline 18 & University of Texas-Austin (Cockrell) & No & 0 & No undergraduate program \\
\hline 19 & University of Wisconsin--Madison & Yes & 4 & http://www.engr.wisc.edu/cmsdocuments/isye-curriculum-2014.pdf \\
\hline 20 & Virginia Tech & Yes & 3 & http://www.ise.vt.edu/UndergradProgram/ImportantDocuments/2013_14__G__Handbook.pdf \\
\hline
\end{tabular}

but they never exceed more than one week. Thus, less than two weeks and a semester exposure to simulation is where college graduates start work as simulation analysts.

We also meet many simulation modelers whose backgrounds are not as industrial or systems engineers. They just take two courses from software vendors and then learn the methodologies further while they work. In fact, I have been somewhat surprised to witness many mistakes and misusage even with simple built-in functions and add-ons. For instance, one input analyzer (i.e., Stat:fit) indicates that there is no good fit for the collected data, but the modeler just used the first distribution that was addressed. If she or he decides to use it as a second best option after carefully comparing it with other distributions, it might be acceptable even though this practice is not recommended. However, if the modeler just chooses the first one listed out of input analyzer, this attitude should be remedied.

In addition, building a simulation model as a team is sometimes considered a norm, but this process should be checked by another modeler. At least another input analyzer such as 
Expert:fit provides qualitative statements indicating that considering empirical distribution or expertise knowledge is a better solution. However, some software just implement their own function to assign input distributions that are seemingly workable but does not assure proper fitting of input data. The reason why I address the cases of input analysis is that while people believe they follow the standard and reliable steps to analyze input data, many errors prevail. Thus, the right usage of output analysis must be strictly enforced as there is no standard method across software environments. We will take a look at different approaches to deal with warm-up period across multiple simulation software to apply MSER methodology to their output analysis. 